\title{
ERMÜDUNGSVERHALTEN VON STAHLGUSSKNOTEN IN BRÜCKEN AUS STAHLHOHLPROFILEN
}

\author{
THÈSE NO 3274 (2005) \\ PRÉSENTÉE À LA FACULTÉ ENVIRONNEMENT NATUREL, ARCHITECTURAL ET CONSTRUIT \\ Institut de structures \\ SECTION DE GÉNIE CIVIL \\ ÉCOLE POLYTECHNIQUE FÉDÉRALE DE LAUSANNE \\ POUR L'OBTENTION DU GRADE DE DOCTEUR ÈS SCIENCES
}

PAR

Senta Christine HALDIMANN - STURM

ingénieure civile diplômée EPF

et de nationalité allemande

acceptée sur proposition du jury:

Prof. M.A. Hirt, directeur de thèse

Dr S. Herion, rapporteur

Prof. A. Mortensen, rapporteur

Prof. M. Schlaich, rapporteur 



\section{ZUSAMMENFASSUNG}

Räumliche Fachwerkkonstruktionen finden auf Grund ihrer konstruktiven und gestalterischen Vorteile im modernen Brückenbau zunehmend Verwendung. Das Konzept des Stahlgussknotens für die Verbindung von Rundhohlprofilen in Fachwerkknoten setzt sich allmählich durch. Für die Bemessung der Fachwerkknoten unter Ermüdungsbelastung sind jedoch weitere Erkenntnisse zum Ermüdungswiderstand notwendig. Die vorliegende Arbeit befasst sich mit dem globalen Ermüdungsverhalten von Stahlgussknoten in den Fachwerklängsträgern von Stahl-Beton-Verbundbrücken.

Anhand experimenteller Untersuchungen wird das globale Ermüdungsverhalten von Stahlgussknoten in einem Fachwerkträger quantitativ erfasst. In Abhängigkeit verschiedener Parameter wird der relative Einfluss der Beanspruchbarkeit des Stahlgussknotens und der Beanspruchbarkeit der Anschlussnähte auf das globale Ermüdungsverhalten untersucht. Eine wirtschaftlich optimale Bemessung eines Stahlgussknotens ist dann gegeben, wenn der Ermüdungswiderstand des Gussknotens demjenigen der Anschlussnähte entspricht. Die Versuchsergebnisse zeigen eindeutig, dass die Schweissnahtanschlüsse für die globale Ermüdungsfestigkeit massgebend sind. Ihr Ermüdungsverhalten wird daher in einem zeitgleich laufenden Forschungsprojekt detailliert untersucht. Die vorliegende Arbeit konzentriert sich auf die Abstimmung der Ermüdungsfestigkeit der Stahlgussknoten auf diejenige der Anschlussschweissnähte. Es sollen also die maximal zulässigen Anfangsgrössen der Gussfehler unter Berücksichtigung der Ermüdungsfestigkeit der Anschlussschweissnähte bestimmt werden.

Diese werden mit Hilfe eines numerischen Modells mit Randelementen für Stahlgussknoten einer typischen Stahl-Beton-Verbundbrücke berechnet. Die Ergebnisse zeigen, dass Sprödbruch des rissbehafteten Knotens ausgeschlossen werden kann. Als Bruchkriterium wird daher eine Risstiefe von $90 \%$ der Wanddicke an der Fehlerstelle festgelegt, da ein Wanddurchbruch des Risses als nicht tolerierbar eingestuft wird. Die maximal zulässigen Anfangsfehlergrössen sind im Verhältnis zur Wanddicke durchwegs sehr gross, die Spanne reicht von 28 bis $88 \%$ der Wanddicke.

Mit dem Ziel einer allgemeinen Anwendung für Stahl-Beton-Verbundbrücken werden die Ergebnisse der numerischen Untersuchungen durch eine auf einem konstanten Korrekturfaktor beruhende Näherungsformel für den Spannungsintensitätsfaktor umschrieben. Dank der vorgeschlagenen Näherungsformel vereinfacht sich das anhand der oben erwähnten typischen Verbundbrücke dargestellte Bemessungsvorgehen für den Ermüdungswiderstand von Stahlgussknoten wesentlich. Diese Näherungsformel dient anschliessend zur Durchführung einer Parameterstudie. Der Einfluss des Ausnutzungsgrades unter Verkehrs- und Ermüdungslast, der Bruchzähigkeit und der Fliessgrenze des Stahlgusses sowie der Knotenabmessungen auf die zulässige Fehlergrösse wird beschrieben. Unter der Annahme einer plausiblen Bruchzähigkeit und einer mittleren Ausnutzung des Knotens werden für einen gewählten Abmessungsbereich die maximal zulässigen Anfangsgrössen von Gussfehlern angegeben.

Diese Arbeit zeigt, dass die Ermüdungsfestigkeit der Anschlussschweissnähte wesentlich verbessert werden muss, um die tatsächlich vorhandene Ermüdungsfestigkeit der Stahlgussknoten ausnützen zu können. Die Anschlussnähte mit Schweissbadsicherung zeichnen sich im Vergleich zu denjenigen ohne durch eine höhere Ermüdungsfestigkeit aus. Mit Hilfe des vorgeschlagenen Bemessungskonzepts können zulässige Anfangsgrössen von Gussfehlern für eine erforderliche Ermüdungsfestigkeit von Stahlgussknoten abgeschätzt werden. 


\section{SUMMARY}

Due to their aesthetic and structural advantages, tubular space truss structures are enjoying increasing popularity in modern bridge construction. The use of cast steel nodes for the joints between the circular hollow section members is also becoming increasingly popular. The fatigue design of such joints however requires additional knowledge with respect to their fatigue resistance. The present work deals with the global fatigue behaviour of cast steel nodes used in longitudinal truss girders of steel-concrete composite bridges.

The global fatigue behaviour of cast steel nodes in a truss girder is quantified on the basis of experimental investigations. The relative influence of the resistance of the cast steel node and the resistance of the welds is analysed as a function of various parameters. An economically optimal fatigue design consists of adapting the fatigue resistance of the cast node to that of the welds. The experimental results show very clearly that the fatigue behaviour is governed by the welds in all tested configurations. Their fatigue behaviour is therefore investigated in a research program conducted simultaneously. The present work concentrates on the adaptation of the resistance of the cast steel nodes to that of the welds. Consequently, allowable initial casting defect sizes must be defined as a function of the required fatigue resistance of the welds.

Using a numerical boundary element model, they are calculated for cast steel nodes in a typical steel-concrete composite bridge. The results show that brittle failure of a node containing cracks does not occur. A crack depth equal to $90 \%$ of the wall thickness at the crack location is therefore chosen as the failure criterion, assuming that a through-thickness crack is unacceptable. Varying between 28 and $88 \%$ of the wall thickness, the resulting allowable initial casting defect sizes are very large.

Aiming at a general application for steel-concrete composite bridges, the results of the numerical investigations are represented by an approximate formula for the stress intensity factor based on a constant correction factor. This approximation results in a considerable simplification of the procedure for the fatigue design of cast steel nodes established for the aforementioned typical composite bridge. The approximate formula is used to perform a parametric study. The influences of the utilisation ratio under traffic and fatigue loads, the cast steel fracture toughness and yield strength and the node dimensions on the defect size are described. Assuming a fracture toughness likely to be encountered in practice and a mean utilisation ratio of the node as well as a range of node dimensions, the allowable initial casting defect sizes are quantified.

The present work shows that the fatigue resistance of the welded joints needs to be improved substantially in order to benefit from the high fatigue resistance of the cast nodes. Weld details including a backing ring offer a higher fatigue resistance than those without. Using the proposed design concept, allowable initial defect sizes in cast steel nodes can be estimated as a function of the required fatigue resistance. 


\section{RESUME}

Grâce à leurs avantages constructifs et créatifs, l'emploi de treillis spatiaux s'accroît dans la construction des ponts modernes. Le concept des nœuds moulés fabriqués en acier coulé pour la réalisation des jonctions dans un treillis s'impose peu à peu par rapport à d'autres concepts. Cependant le dimensionnement à la fatigue des nœuds moulés exige de plus amples connaissances quant à la détermination de la résistance à la fatigue. Le présent travail traite du comportement global à la fatigue des nœuds moulés dans les poutres maîtresses longitudinales à treillis de ponts mixtes acier-béton.

L'étude expérimentale traite du comportement global à la fatigue des nœuds moulés faisant partie d'une poutre à treillis. L'influence relative des deux aspects « résistance des nœuds moulés » et " résistance des soudures de raccord " sur le comportement global à la fatigue est étudiée en faisant varier différents paramètres. Un dimensionnement économiquement optimal est obtenu lorsque la résistance à la fatigue des nœuds moulés correspond à celle des soudures de raccord. Pour les configurations utilisées lors des essais, les résultats montrent clairement que les soudures sont déterminantes pour la résistance à la fatigue. Pour cette raison, leur comportement à la fatigue est étudié en même temps dans le cadre d'un autre projet de recherche. Le présent travail se concentre sur l'adaptation de la résistance des nœuds moulés à celle des soudures de raccord. Il faut donc définir des tailles maximales admissibles des défauts dans l'acier en fonction de la résistance des soudures de raccord.

A l'aide d'un modèle numérique utilisant les éléments frontières, elles sont calculées pour un exemple de pont mixte acier-béton type. Les résultats montrent que la rupture fragile d'un nœud fissuré peut être exclue pour ce cas. Vue qu'une rupture à travers la paroi du nœud moulé est définie non tolérable, le critère de rupture est ainsi est fixé à $90 \%$ de l'épaisseur de paroi à l'endroit du défaut. Les tailles maximales admissibles obtenues sont très grandes par rapport à l'épaisseur de paroi à l'endroit du défaut. Elles valent entre 28 et $88 \%$ de l'épaisseur de paroi.

Dans le but d'une application plus générale pour les ponts mixtes acier-béton, on montre que les résultats de l'étude numérique peuvent être décrits par une approximation du facteur d'intensité de contraintes, où le facteur de correction est constant. Cette formulation approximative simplifie le procédé du dimensionnement à la fatigue des nœuds moulés établie pour l'exemple de pont mixte type et sert ensuite à effectuer une étude paramétrique. L'influence du taux d'utilisation de l'acier sous charges de trafic et sous charges de fatigue, de la ténacité et de la limite d'élasticité de l’acier coulé ainsi que de la géométrie du nœud moulé sur la taille admissible du défaut est décrite. En prenant une ténacité plausible, les tailles maximales admissibles des défauts dans l'acier coulé sont quantifiées pour un taux d'utilisation moyen du nœud moulé ainsi que pour une étendue choisie de la géométrie du nœud.

Les conclusions les plus importantes montrent que la résistance des soudures entre le nœud moulé et les tubes doit être nettement améliorée afin de pouvoir exploiter la résistance effectivement disponible des nœuds moulés. Les soudures avec une liste de soudage se distinguent - en comparaison à celles sans liste - par une meilleure résistance à la fatigue. A l'aide d'un concept de dimensionnement, pour une résistance à la fatigue des nœuds moulés requise, les tailles maximales admissibles des défauts dans l'acier coulé peuvent être évaluées. 



\section{VERDANKUNG}

An erster Stelle danke ich meinem Doktorvater Herrn Prof. Manfred A. Hirt, Leiter des Lehrstuhls für Stahlbau ICOM, der mich motiviert, mir das Vertrauen entgegengebracht und mich stets unterstützt hat, diese Arbeit durchzuführen. An seinem Lehrstuhl hat er mir die besten Voraussetzungen für das Gelingen dieser Arbeit geboten.

Mein besonderer Dank geht an Herrn Dr. Alain Nussbaumer für die wissenschaftliche Betreuung dieser Arbeit. Unsere gemeinsamen, zahlreichen und sehr anregenden Diskussionen, die manchmal auch vom Fachlichen abwichen, seine Erfahrungen in der Durchführung von Ermüdungsversuchen, sein fachspezifisches Wissen sowie seine kritischen Bemerkungen und Ideen habe ich sehr geschätzt.

Ich möchte dem Schweizerischen Nationalfonds und der FOSTA-Forschungsvereinigung Stahlanwendung e.V. für die finanzielle Unterstützung der Arbeit danken. Brütsch/Rüegger AG (Schweiz) und Vallourec \& Mannesmann Tubes (Deutschland) danke ich für die grosszügige Bereitstellung der Rundhohlprofile für die Probekörper. Ich danke ebenfalls der Friedrich Wilhelms-Hütte GmbH für die Mitfinanzierung der Stahlgussknoten.

Mein Dank gilt den Mitgliedern der Prüfungskommission (in alphabetischer Reihenfolge) unter der Leitung von Herrn Prof. Anton Schleiss (EPFL, Laboratoire de constructions hydrauliques), Herrn Dr. Stefan Herion (Universität Karlsruhe, Versuchsanstalt für Stahl, Holz und Steine), Herrn Prof. Andreas Mortensen (EPFL, Laboratoire de métallurgie mécanique) und Herrn Prof. Mike Schlaich (Technische Universität Berlin, Institut für Bauingenieurwesen, Fachgebiet Massivbau) für ihre Bereitschaft, das Gutachten meiner Dissertation zu übernehmen, für ihre wertvollen Kommentare, Anmerkungen und Verbesserungsvorschläge für die Endversion des vorliegenden Dokuments und für ihr Verständnis für die Verschiebung der Doktoratsprüfung auf Grund meiner Schwangerschaftsprobleme.

Ebenfalls möchte ich mich bei den Mitgliedern meiner Begleitkommission (in alphabetischer Reihenfolge) bedanken, Herrn Dr. Hans-Gerhard Dauner, Herrn Prof. Luc Espic (La Haute Ecole d'Ingénieurs et de Gestion du Canton de Vaud), Herrn Dr. Bertram Kühn (PSP Prof. Sedlacek \& Partner GmbH) und Herrn Prof. Andreas Mortensen (EPFL, Laboratoire de métallurgie mécanique) für die regen und hilfreichen Diskussionen und Vorschläge während den halbjährlichen Treffen.

Im Verlauf der Arbeit haben mir ausserhalb des Lehrstuhls für Stahlbau ICOM zahlreiche Personen mit Rat und Tat beigestanden. Insbesondere folgenden Personen möchte ich danken: Herrn Karsten Heßeln und Herrn Karl-Josef Müller (Friedrich Wilhelms-Hütte GmbH) für die Beratung bei der Herstellung der Stahlgussknoten, Herrn Ahmed Shah Rafiq und Herrn Claudio Brutschi (JAKEM AG), Herrn Ulrich Hadrian (Schweizerischer Verein für Schweisstechnik) und Herrn Prof. André Flückiger (La Haute Ecole d'Ingénieurs et de Gestion du Canton de Vaud) für die Beratung bei der Herstellung der Probekörper, Frau Susanne Höhler (RWTH Aachen, Lehrstuhl für Stahlbau) für ihre geduldige Einführung zur Benutzung des Programms BEASY, Herrn Jean-Pierre Pollien (Quality Control NDT S.A.) für die lehrreichen Diskussionen über die Grenzen der Ultraschalluntersuchungen und Herrn Markus Gottier (Gottier Engineering) für die Diskussionen über die Anwendung der Bruchmechanik.

Mein Dank geht auch an Herrn Sylvain Demierre, Herrn Gilbert Pidoux und Herrn Hans-Jakob Reist, die den Versuchsaufbau realisierten und mir bei all den unerwartenden Problemen tatkräftig zur Seite standen. 
Für die organisatorischen, administrativen und graphischen Arbeiten möchte ich mich ebenfalls bei Frau Esther von Arx und Herrn Claudio Leonardi bedanken.

Am Lehrstuhl für Stahlbau ICOM habe ich während meines Aufenthalts nicht nur sehr gute Arbeitskollegen gefunden sondern auch Freundschaften geschlossen. Allen voran möchte ich mich bei meiner Vorgängerin in der Forschung an Knoten in Rohrfachwerkbrücken, Frau Dr. Ann Schumacher, für ihre herzliche und offene Art sowohl im Büro als auch ausserhalb der Bürozeiten bedanken. Mein Dank geht auch an meine beiden Kollegen der Gruppe Fatigue, Herrn Dr. Scott Walbridge und Herrn Luis Borges, für den Austausch von Erfahrungen und Tipps zur Ermüdungsproblematik, Englischkorrektur und BEASY-Anwendungen. Bei meinen Bürokollegen, Herrn Dr. Andrea Bassetti, Herrn Stéphane Utz, Frau Martina Eliášová und Herrn Yves Rey möchte ich mich ebenfalls für die angenehme Arbeitsatmosphäre bedanken. Für die schöne Zeit am ICOM und auch während den zahlreichen Unternehmungen möchte ich mich bei Abeer, Ahti, Alain, Ann, Andreas, Bertram, Christoph, Danijel, François, Jean-Paul, Luis, Michel C., Michel T., Rahel, Scott, Thierry, Thomas und Yves bedanken.

Ich möchte mich auf das Herzlichste bei meinen Eltern und bei meiner Grossmutter Christel für ihr allgegenwärtiges Interesse an meiner Arbeit und ihre grosse Motivation und Unterstützung während der langen Ausbildungsjahre bedanken. Meiner Mutter, meiner Schwiegermutter und meiner Schwägerin Mirjam möchte ich aufrichtig danken, dass sie sich während der Vorbereitung meiner Doktoratsprüfung oft um unsere kleine Vera kümmern konnten.

Mein allergrösster Dank geht an meinen Ehemann Matthias für seinen moralischen Beistand und seinen glühenden Ansporn in schwierigen Momenten, für sein kritisches Korrekturlesen dieser Arbeit, für seine stete Hilfe bei Computerproblemen und insbesondere für die wunderschöne gemeinsame Zeit, seit wir uns am Lehrstuhl für Stahlbau ICOM kennen gelernt haben. 


\section{INHALT}

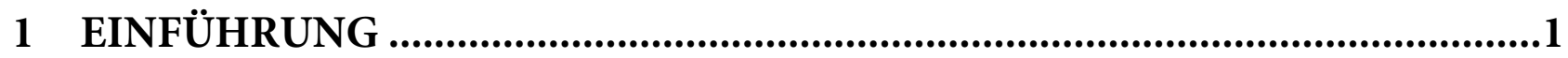

1.1 Motivation ………....................................................................................... 1

1.2 Problemstellung............................................................................................. 2

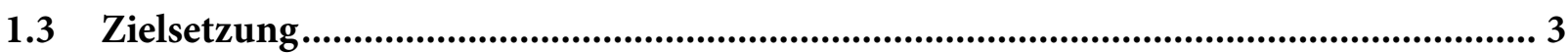

1.4 Vorgehensweise …………………........................................................................ 3

1.5 Aufbau der Arbeit............................................................................................................ 5

2 WISSENSSTAND ZUR ANWENDUNG VON STAHLGUSSKNOTEN ............7

2.1 Stahlgussknoten als Konstruktionsdetail - Entwicklung ….......................................... 7

2.1.1 Offshore-Bauwerke ...........................................................................................................

2.1.2 Stahlhoch- und Brückenbau ..........................................................................................

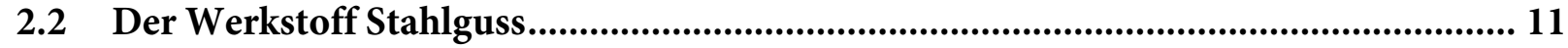

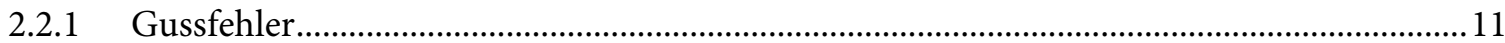

2.2.2 Nachweis von Gussfehlern........................................................................................... 14

2.2.3 Liefernorm für Stahlgusskomponenten ........................................................................... 16

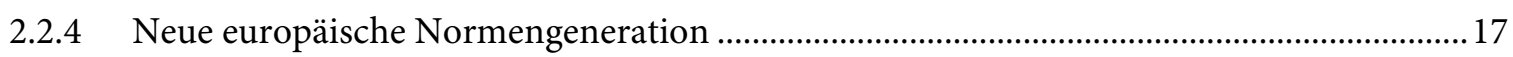

2.3 Ermüdungsbemessung von Stahlgussknoten .............................................................. 18

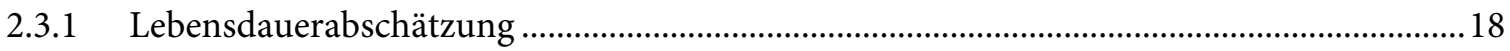

2.3.2 Berechnung der Spannungskonzentrationsfaktoren.........................................................22

2.4 Ermüdungsbemessung von Schweissnahtanschlüssen bei Stahlgussknoten .............. 22

2.4.1 Abschätzung der Lebensdauer mit Ermüdungsfestigkeitskurven ..........................................22

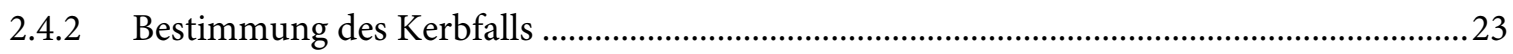

2.4.3 Berechnung der Spannungskonzentrationsfaktoren................................................................26

2.5 Zusammenfassung und Folgerungen .......................................................................... 27

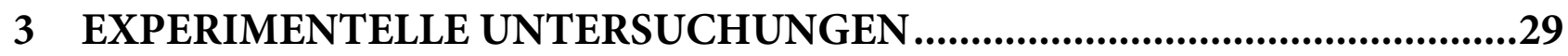

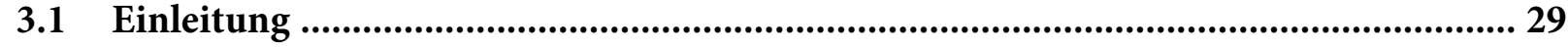

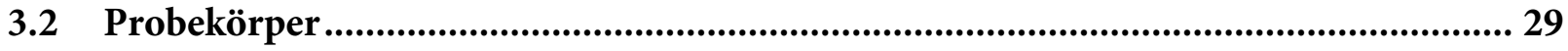

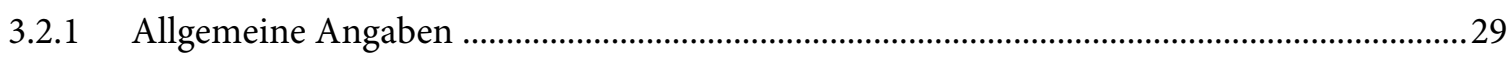

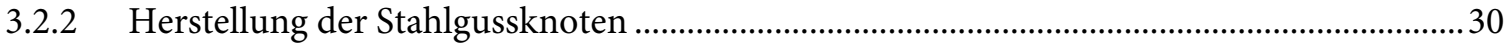

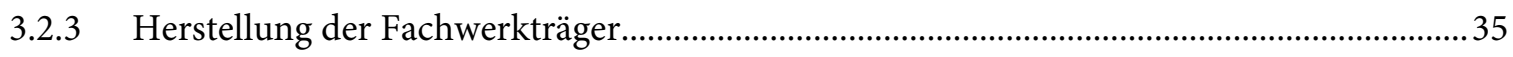

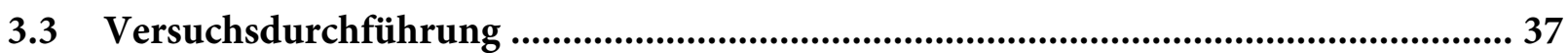

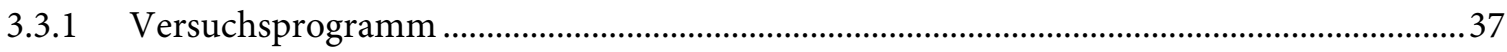

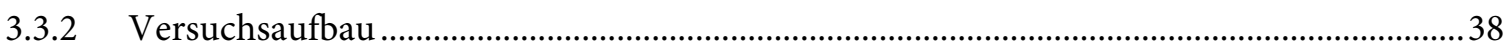

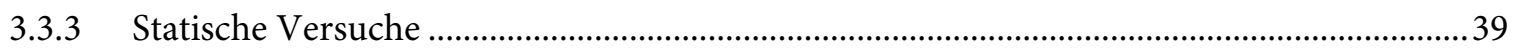

3.3.4 Ermüdungsversuche ………………………................................................................ 39

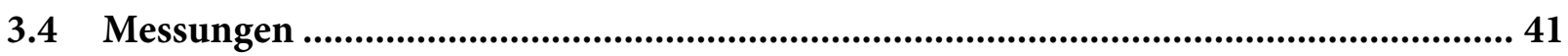


3.4.1 Messungen unter statischer Last ...................................................................................... 41

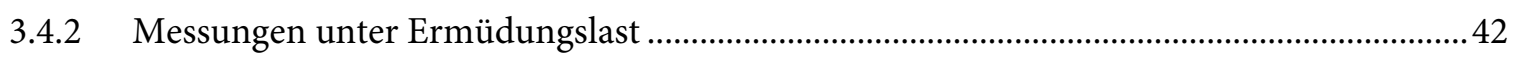

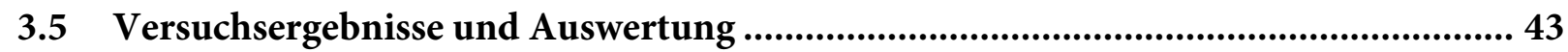

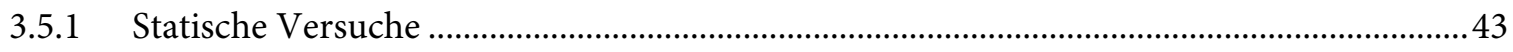

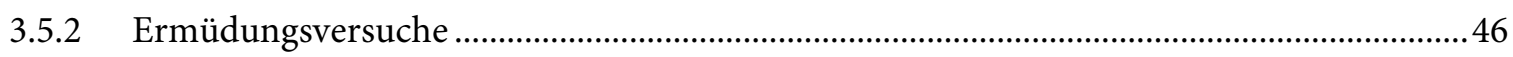

3.5.3 Überprüfung der Stahlgussknoten auf Gussfehler und Ermüdungsrisse ................................56

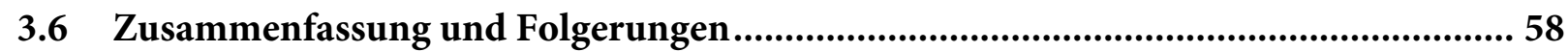

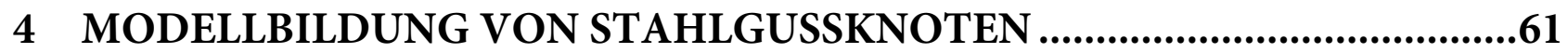

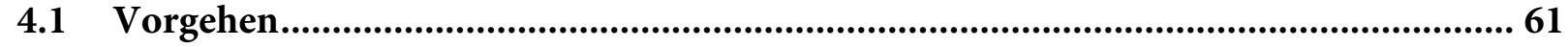

4.2 Modellierung mit finiten Elementen (FE) ................................................................ 61

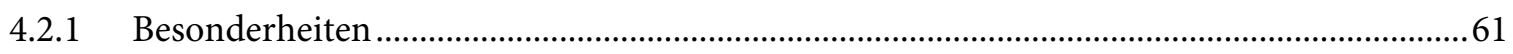

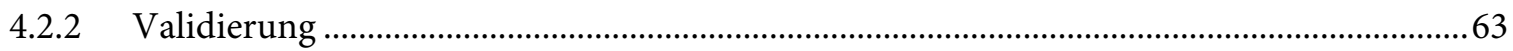

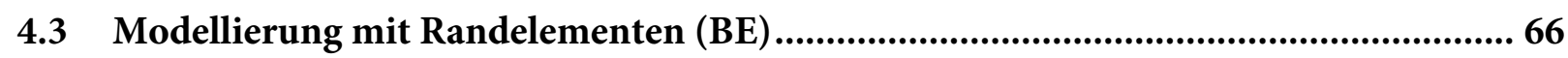

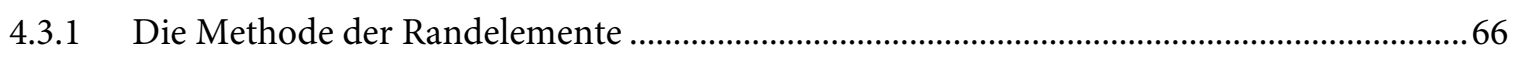

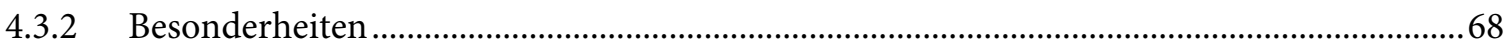

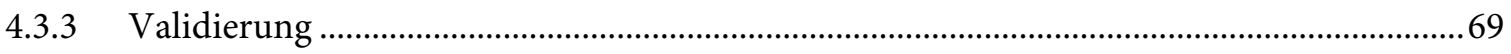

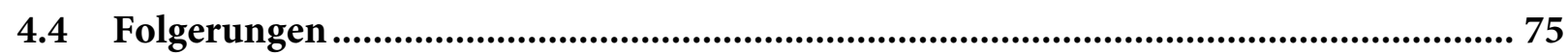

\section{QUALITÄTSANFORDERUNGEN AM BEISPIEL EINER}

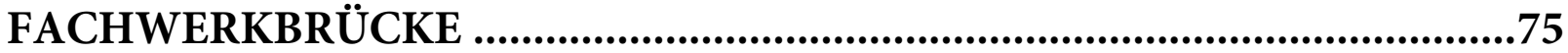

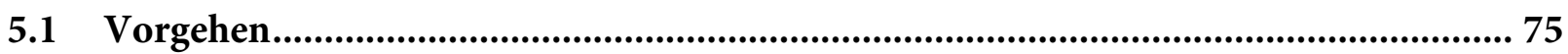

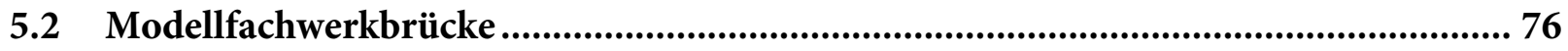

5.2.1 Beschreibung ............................................................................................................ 76

5.2.2 Form der Stahlgussknoten für die Modellfachwerkbrücke ................................................... 78

5.2.3 Modellierung der Stahlgussknoten im Stabmodell der Brücke ................................................79

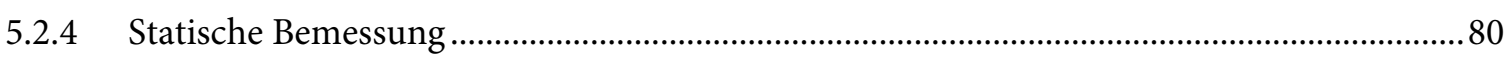

5.2.5 Ermüdungsbemessung ............................................................................................... 80

5.2.6 Ergebnisse aus statischer Bemessung und Ermüdungsbemessung ........................................ 81

5.3 Belastungszustände der Stahlgussknoten ................................................................... 82

5.3.1 Wahl der zu untersuchenden Knoten ................................................................................... 82

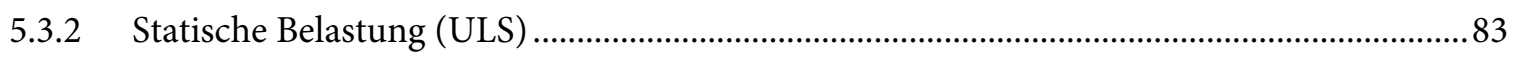

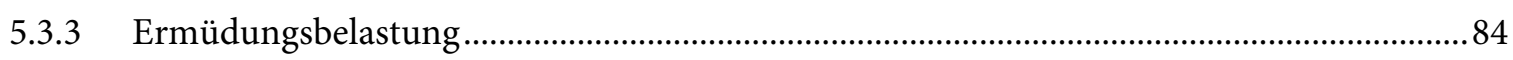

5.4 Rückrechnung auf maximal zulässige Anfangsfehlergrösse ....................................... 91

5.4.1 Berechnungsannahmen.................................................................................................... 91

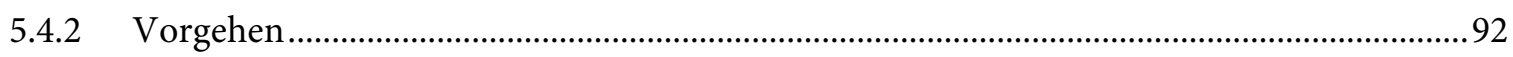

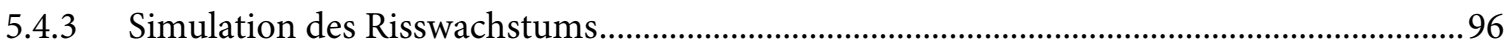

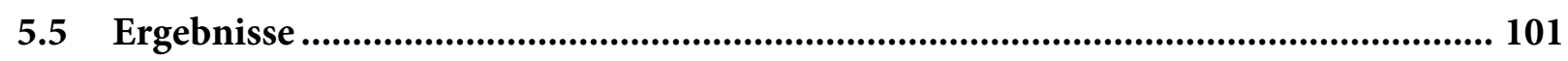

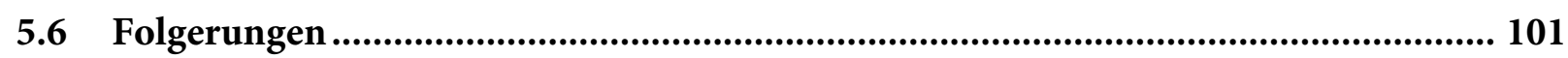


6 VERALLGEMEINERUNG UND PARAMETERSTUDIE ...........................103

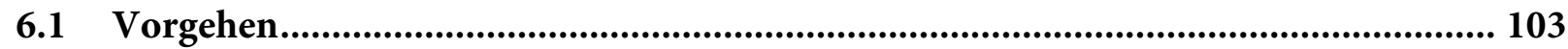

6.2 Näherungsformel für den Spannungsintensitätsfaktor ........................................ 104

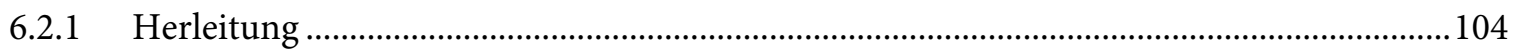

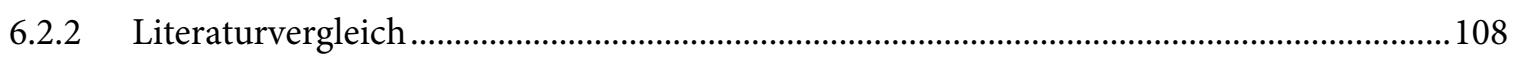

6.2.3 Überprüfung anhand der Modellfachwerkbrücke........................................................... 110

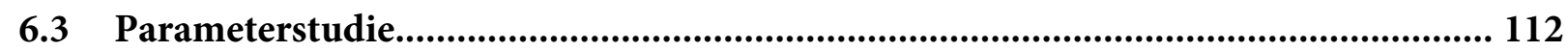

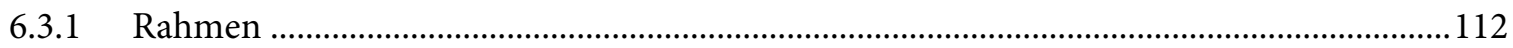

6.3.2 Algorithmus zur Berechnung der zulässigen Anfangsfehlergrösse......................................... 114

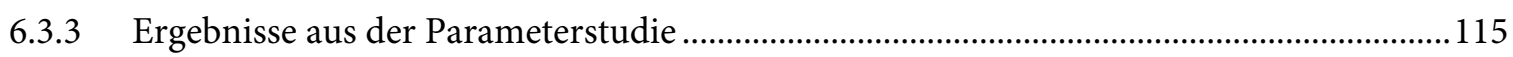

6.4 Vorgehen für die Ermüdungsbemessung …................................................... 124

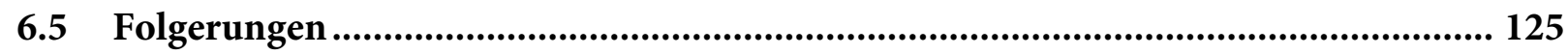

7 ZUSAMMENFASSUNG, FOLGERUNGEN UND WEITERFÜHRENDE

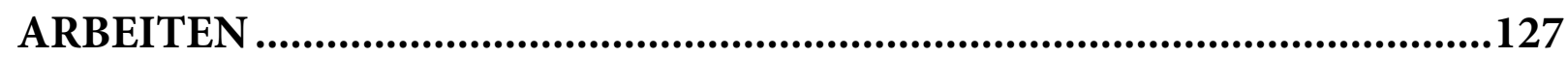

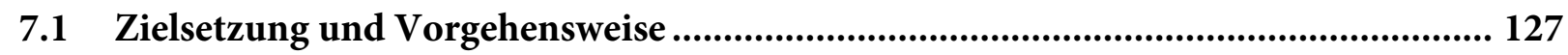

7.2 Zusammenfassung und Folgerungen......................................................................... 128

7.3 Schlussfolgerungen .................................................................................................. 131

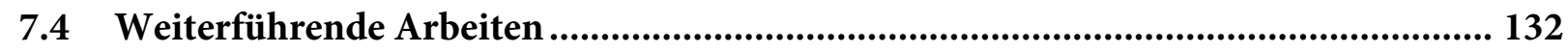

LITERATURVERZEICHNIS .................................................................135

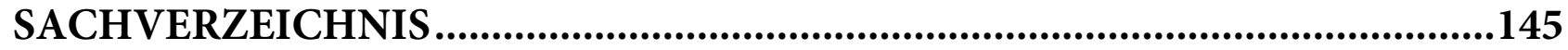

\section{INHALT ANHÄNGE}

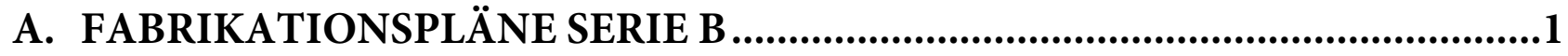

B. TABELLARISCHE AUFLISTUNG DER VERSUCHSDATEN .............................3

C. ALGORITHMUS ZUR BERECHNUNG DER MAXIMAL ZULÄSSIGEN ANFANGSFEHLERGRÖSSE. .4

\section{CURRICULUM VITAE}





\section{NOTATION}

\section{GROSSBUCHSTABEN}
$A_{\mathrm{v}}$
Kerbschlagarbeit
C
Rissfortschrittsparameter
$D$
Aussendurchmesser des Untergurts (Rundhohlprofil) in einem Fachwerk- träger
E
Elastizitätsmodul
$J_{\text {Ic }}$
kritischer Wert des J-Integrals bei dem es zur Rissausbreitung kommt
$K_{\mathrm{I}}, K_{\mathrm{II}}, K_{\mathrm{III}}$
Spannungsintensitätsfaktor für den Modus I, II bzw. III
$K_{\text {Ic }}$
Bruchzähigkeit des Materials für den Modus I bei ebenem Dehnungszustand
$K_{\mathrm{I}, \text { Eigengewicht }}$
Spannungsintensitätsfaktor für den Modus I unter Eigengewicht
$K_{\mathrm{I}, \mathrm{ULS}}, K_{\mathrm{II}, \mathrm{ULS}}, K_{\mathrm{III}, \mathrm{ULS}}$
Spannungsintensitätsfaktor für den Modus I, II bzw. III unter statischer Last
$\hat{K}_{\text {I }}^{\mathrm{a}}$
normierter Spannungsintensitätsfaktor für den Modus I in der Risstiefe
$K_{\mathrm{I}}^{\mathrm{a}}$
Spannungsintensitätsfaktor für den Modus I in der Risstiefe
$\hat{K}_{\mathrm{I}}^{\mathrm{c}}$
normierter Spannungsintensitätsfaktor für den Modus I am Rissrand
$K_{\mathrm{I}}^{c}$
Spannungsintensitätsfaktor für den Modus I am Rissrand
$K_{\mathrm{I}, \mathrm{ULS}}^{\mathrm{c}}$
Spannungsintensitätsfaktor für den Modus I am Rissrand unter statischer Last
$K_{\mathrm{I}, \max }$
maximaler Spannungsintensitätsfaktor für Modus I
$K_{\mathrm{I}, \min }$
minimaler Spannungsintensitätsfaktor für Modus I
$K_{\text {op }}$
Spannungsintensitätsfaktor bei dem die Risslippen tatsächlich geöffnet wer- den
$K_{\text {ULS }} \quad$ Spannungsintensitätsfaktor unter statischer Last
$N$
Anzahl Lastwechsel
Q
Last
$Q_{\min }$
minimale Ermüdungslast
$Q_{\max }$
maximale Ermüdungslast
$R_{\mathrm{i}}$
Innenradius
$R_{\mathrm{m}}$
mittlerer Radius
$R^{2}$
Determinationskoeffizient, Bestimmtheitsmass
$S$
Dehnungsenergiedichte
$T$
Wanddicke des Untergurts (Rundhohlprofil) eines Fachwerkträgers 


$\begin{array}{ll}T_{0} & \text { Bezugstemperatur } \\ T_{\text {Test }} & \text { Prüftemperatur } \\ Y & \begin{array}{l}\text { konstanter Korrekturfaktor bei der Berechnung des Spannungsintensitäts- } \\ \text { faktors } \\ \text { konstanter Korrekturfaktor für den Spannungsintensitätsfaktor in der Riss- } \\ Y_{\text {a }}\end{array} \\ Y_{\mathrm{c}} & \text { kiefe } \\ & \text { konstanter Korrekturfaktor für den Spannungsintensitätsfaktor am Rissrand }\end{array}$

\section{KLEINBUCHSTABEN}

\begin{tabular}{|c|c|}
\hline$a$ & Risstiefe \\
\hline$a_{0}$ & maximal zulässige Anfangsfehlergrösse eines Gussfehlers \\
\hline$a_{0, i}$ & $\begin{array}{l}\text { maximal zulässige Anfangsfehlergrösse eines Gussfehlers an der Fehlerstelle } \\
i\end{array}$ \\
\hline$a_{0, i, \mathrm{BE}}$ & $\begin{array}{l}\text { maximal zulässige Anfangsfehlergrösse eines Gussfehlers an der Fehlerstelle } \\
i \text {, mit dem Programm BEASY berechnet }\end{array}$ \\
\hline$a_{\text {eff }}$ & $\begin{array}{l}\text { effektive Risstiefe unter Berücksichtigung der plastischen Zone um die } \\
\text { Risspitze }\end{array}$ \\
\hline$a_{\text {krit }}$ & $\begin{array}{l}\text { im Failure Assessment Diagramm ermittelte kritische Fehlergrösse für } \\
\text { Sprödbruch }\end{array}$ \\
\hline$a_{\text {mass }}$ & $\begin{array}{l}\text { massgebende Fehlergrösse für die Rückrechnung auf die maximal zulässige } \\
\text { Anfangsgrösse eines Gussfehlers }\end{array}$ \\
\hline$a_{\text {mass }, i}$ & massgebende Fehlergrösse an der Fehlerstelle $i$ \\
\hline$b$ & 2. Regressionskoeffizient (Ordinatenabschnitt); Plattenlänge \\
\hline$c$ & halbe Risslänge \\
\hline$d$ & Aussendurchmesser der Diagonle (Rundhohlprofil) eines Fachwerkträgers \\
\hline$e$ & Exzentrizität zwischen den Wanddickenmitten von Blechen \\
\hline$f_{\mathrm{u}}$ & Zugfestigkeit \\
\hline$f_{\mathrm{y}}$ & Fliessgrenze \\
\hline$k_{\mathrm{s}}$ & Korrekturfaktor \\
\hline$m$ & Rissfortschrittsparameter \\
\hline$s_{\mathrm{N}}$ & Standardabweichung \\
\hline$t$ & $\begin{array}{l}\text { Wanddicke der Diagonale (Rundhohlprofil) eines Fachwerkträgers; allge- } \\
\text { mein Wanddicke }\end{array}$ \\
\hline$u, v, w$ & Verschiebung in Richtung $x, y, z$-Richtung \\
\hline
\end{tabular}


$w$

$w_{i}$

Wanddicke im Stahlgussknoten

Wanddicke an der Fehlerstelle $i$ im Stahlgussknoten

\section{GROSSE GRIECHISCHE BUCHSTABEN}

$\Delta K_{\mathrm{I}}$

$\Delta K_{\text {eff }}$

$\Delta K_{\text {th }}$

$\Delta Q$

$\Delta T$

$\Delta \sigma_{1, \mathrm{E} 2}$

$\Delta \sigma_{\mathrm{C}}$

$\Delta \sigma_{\mathrm{D}}$

$\Delta \sigma_{\mathrm{SCF}}$

$\Phi$

$\Phi_{2}$

$\Phi_{3}$

$\Psi_{1}$
Differenz des Spannungsintensitätsfaktors für den Modus I

effektive Differenz des Spannungsintensitätsfaktors

Schwellenwert der Differenz des Spannungsintensitätsfaktors

Schwingbreite der Last $Q$

Temperaturdifferenz

Schwingbreite der ersten Hauptspannung infolge Ermüdungslast

Ermüdungsfestigkeit bei $2 \cdot 10^{6}$ Lastwechseln, Kerbgruppe

Dauerfestigkeit

Spannungsschwingbreite unter Berücksichtigung der Spannungskonzentration

Winkel, mit dem jede Position entlang der Rissfront beschrieben wird $(0 \leq \Phi \leq \pi)$

Rotation um die $y$-Achse

Rotation um die $z$-Achse

Reduktionsbeiwert für den häufigen Wert einer veränderlichen Einwirkung

\section{KLEINE GRIECHISCHE BUCHSTABEN}

$\alpha$

$\beta$

$\gamma$

$\gamma_{\mathrm{F}}$

$\gamma_{G}$

$\gamma_{\mathrm{Mf}}$

$\gamma_{Q}$

$\mathcal{E}$

$\mathcal{E}_{\mathrm{ax}}$

$\mathcal{E}_{\mathrm{b}}$

$\varepsilon_{\text {nom }}$

$\mathcal{E}_{\mathrm{u}}$

Temperaturausdehnungskoeffizient

geometrischer Parameter: $\beta=d / D$

geometrischer Parameter: $\gamma=D / 2 w$

Lastbeiwert

Lastbeiwert für eine ständige Einwirkung

Widerstandsbeiwert der Ermüdungsfestigkeit

Lastbeiwert für eine veränderliche Einwirkung

Dehnung

Dehnung aus Normalkraft

Dehnung aus Biegemoment

Nominaldehnung

Bruchdehnung 
halber Rissöffnungswinkel eines Durchrisses im Rundhohlprofil; Öffnungswinkel der Diagonalen in einem Fachwerkträger

$\theta_{0}$

halber Rissöffnungswinkel nach dem Übergang vom Oberflächenriss zum Durchriss

$\theta_{\max }$

maximaler halber Rissöffnungswinkel

$\kappa$

Multiplikationsfaktor

$\sigma$

Spannung

$\sigma_{1}$

erste Hauptzugspannung

$\sigma_{1, \mathrm{Ed}}$

erste Hauptzugspannung infolge Verkehrslast als Begleiteinwirkung (Bauteiltemperatur als Leiteinwirkung)

$\sigma_{\text {Bezug }}$

Nominalspannung im rissbehafteten Querschnitt

$\sigma_{\mathrm{f}}$

Mittelwert aus Fliessgrenze und Zugfestigkeit

$\hat{\sigma}_{1}$

Einheitsspannung in Richtung der ersten Hauptspannung

$\tau_{1}$

Wanddickenverhältnis Stutzen- zu Hohlprofildicke der Diagonale im Stahlgussknoten

$\tau_{2}$

Wanddickenverhältnis Stutzen- zu Hohlprofildicke des Untergurts im Stahlgussknoten

$v$

Querdehnungszahl

$\chi_{\mathrm{E}}$

Ausnutzung unter Ermüdung

$\chi_{\mathrm{V}}$

Ausnutzung unter Verkehr 


\section{$1 \quad$ EINFÜHRUNG}

\section{$1.1 \quad$ MOTIVATION}

Im modernen Brückenbau finden räumliche Fachwerkkonstruktionen aus Stahlhohlprofilen auf Grund ihrer konstruktiven und gestalterischen Vorteile zunehmend Verwendung. Stahlhohlprofile mit Kreisquerschnitt geben der Konstruktion weiche und kantenlose Linien und lassen sie geschlossen erscheinen. Der Einsatz von Rundhohlprofilen an Stelle der weit verbreiteten offenen Stahlbauprofile ermöglicht eine gute Lastabtragung und einen verbesserten Korrosionsschutz. Ein effizientes Verhältnis von Tragwiderstand und Eigengewicht (Kosten) wird erreicht durch vergleichsweise günstige Werte in allen Querschnittsachsen.

Die Bemessung und konstruktive Ausbildung der Fachwerkknoten unter Ermüdungsbelastung erfordert neue Erkenntnisse bei der Ermittlung des Ermüdungswiderstands. Es wurden bereits verschiedene konstruktive Konzepte für die Verbindung von Rundhohlprofilen in einem Knoten entwickelt und angewandt, wobei sich die Konzepte des direkt verschweissten Knotens und des Stahlgussknotens im Brückenbau weitgehend durchgesetzt haben. Interessanterweise werden bis heute geschweisste Knoten vorwiegend in der Schweiz [Dauner 1998] [Dauner et al. 1998] [Stucki et al. 2000] angewandt, während Brücken mit Stahlgussknoten vor allem in Deutschland [Schlaich et al. 1999.2] [Schlaich et al. 2000.1] [Bernhardt et al. 2003] und in den Niederlanden [Glijnis et al. 2003] gebaut wurden.

Beim Konzept des geschweissten Fachwerkknotens ist der Gurt des Fachwerkträgers durchlaufend. Die Schweissnahtkanten der Diagonalen werden elektronisch gesteuert zugeschnitten und am Gurt verschweisst. Die räumlichen Beanspruchungen der Knotenschweissnähte, die immer in Bereichen hoher Spannungsspitzen liegen, haben jedoch geringe Ermüdungswiderstände zur Folge. Die Stahlgussknoten sind deshalb eine gute Alternative zur Verbesserung des Ermüdungsverhaltens von Brücken aus Stahlhohlprofilen.
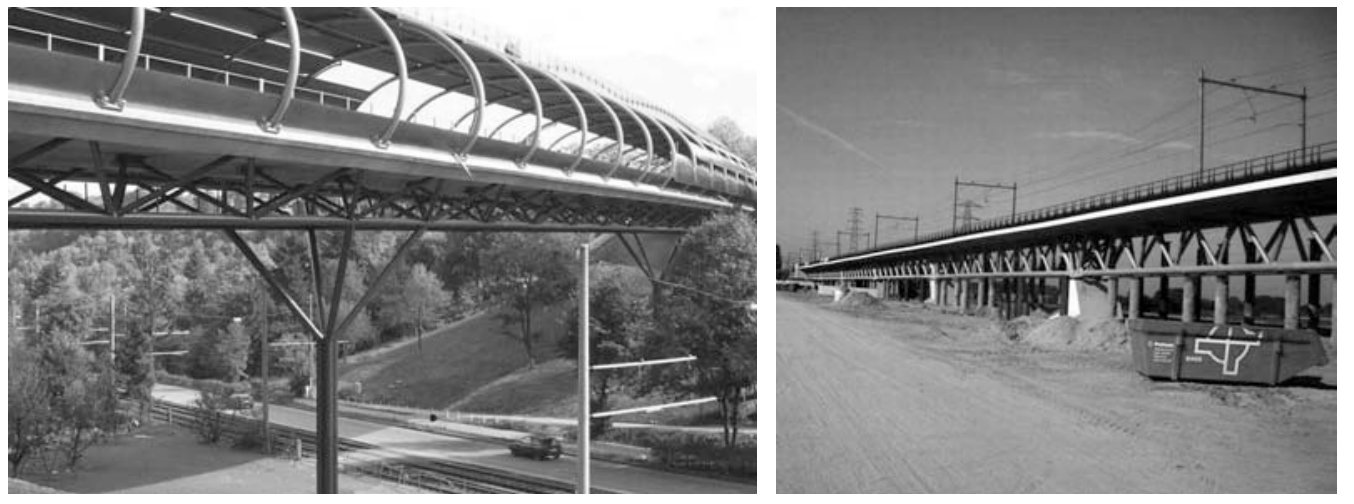

Fig. 1.1: Nesenbachtalbrücke, Stuttgart (D), 1999 Fig. 1.2: Bahnbrücke Oosterbeek (NL), 2002

Erfahrungen aus dem Offshore-Bereich zeigen, dass Stahlguss-Fachwerkknoten gegenüber direkt geschweissten Knoten einen besseren Kraftfluss haben. Die Verlegung der Schweissnähte aus den Bereichen hoher Spannungsspitzen und die Ausführung von Knoten ohne exzentrische Anschlüsse erhöhen den Ermüdungswiderstand des Tragwerks. Zudem ermöglicht die freie Gestaltung der Knotenform einen vielfältigen Einsatz von Stahlgussknoten und Stahlhohlprofilen für verschie- 
denste Brückentypen: Fachwerkbrücken mit gleichschenkligem Dreieck als Querschnitt (Fig. 1.1); Fachwerkbrücken mit einem offenen Querschnitt und zwei ebenen, mit Querstreben verbundenen Fachwerklängsträgern (Fig. 1.2); Bogenbrücken (Fig. 1.3) und Brücken mit als Baumstützen ausgebildeten Pfeilern (Fig. 1.4). Diesen Vorteilen bei der Verwendung von Stahlgussknoten stehen aber auch Nachteile gegenüber. Bei der Verwendung von Stahlgussknoten werden - im Vergleich zu den geschweissten Knoten - die ermüdungsgefährdeten Schweissnähte aus den Bereichen hoher Spannungsspitzen entfernt. Die Verbindungen zwischen dem Stahlgussknoten und den Hohlprofilen im Regelfall Stumpfschweissnähte - sind aber ebenfalls ermüdungsgefährdet. Zudem ist der Untergurt nicht mehr durchlaufend. Dies führt pro Fachwerkknoten zu zwei zusätzlichen, ermüdungsgefährdeten Stumpfschweissnähten.

Ausserdem sind bedingt durch den Herstellungsprozess der Stahlgussknoten im Materialgefüge Gussfehler wie Gasblasen, Schlackeneinschlüsse und Lunker vorhanden. Sie können die Tragfähigkeit und Ermüdungsfestigkeit der Stahlgussknoten wesentlich reduzieren. In der Norm [DIN 16902:1985] werden die verschiedenen Gütestufen für Stahlgusskomponenten definiert, da deren Spezifizierung für die Herstellung unabdingbar ist. Es werden jedoch keine Angaben zur Ermüdungsfestigkeit der jeweiligen Gütestufen gemacht.

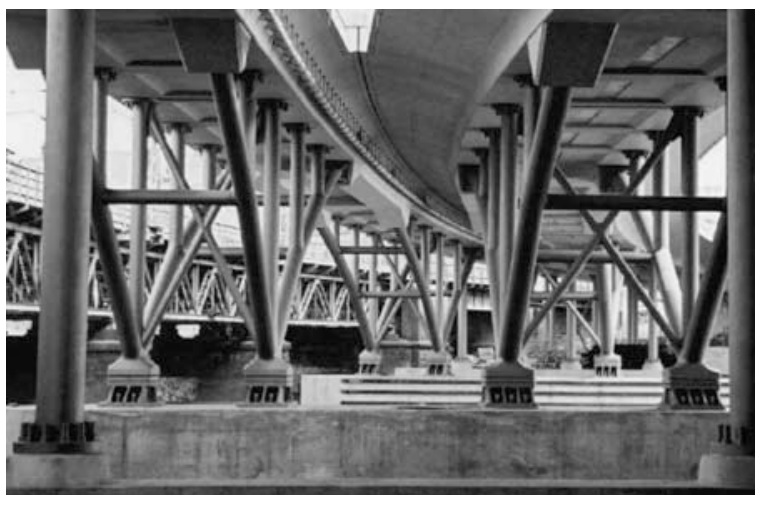

Fig. 1.3: Humboldthafenbrücken,Berlin (D), 2001

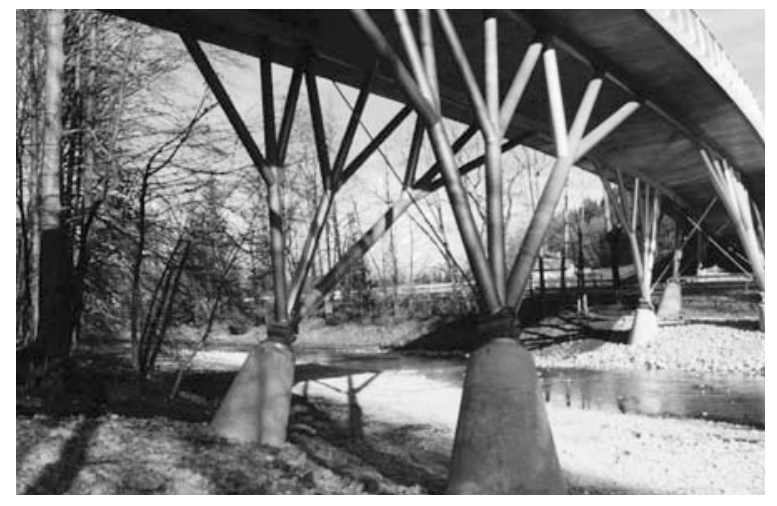

Fig. 1.4: Traunsteinbrücke, Traunstein (D), 2000

Bei den in Fig. 1.1 und Fig. 1.2 abgebildeten Brückentypen ist das Ermüdungsverhalten der Fachwerkuntergurte von grosser Bedeutung. In diesen Bereichen sind die Stahlgussknoten und deren Schweissnahtanschlüsse hohen Zugspannungen ausgesetzt. Bei den Brückentypen nach Fig. 1.3 und Fig. 1.4 werden Druckstäbe an die Gussknoten angeschlossen und die Ermüdungsgefährdung ist von untergeordneter Bedeutung. Die vorliegende Arbeit konzentriert sich daher auf Stahlgussknoten, die für Brückentypen mit einem Fachwerkträger als Hauptlängsträger eingesetzt werden.

\subsection{PROBLEMSTELLUNG}

Die vorliegende Arbeit befasst sich mit dem globalen Ermüdungsverhalten von Stahlgussknoten in den Längsträgern von Stahl-Beton-Verbundbrücken. Dieses wird im Wesentlichen durch zwei Komponenten bestimmt, durch die Ermüdungsfestigkeit des Stahlgussknotens und diejenige der Schweissnahtanschlüsse. Aus wirtschaftlicher Sicht ist es sinnvoll, wenn die Ermüdungsfestigkeiten von Gussknoten und Anschlussnähten gleich gross sind. Eine optimale Ermüdungsbemessung ist dann gegeben, wenn der Ermüdungswiderstand der Stahlgussknoten demjenigen der Anschlussnähte entspricht. 
In der Liefernorm [DIN 1690-2:1985] werden verschiedene Gütestufen für eine Stahlgusskomponente definiert. Für jede Gütestufe sind im Materialgefüge und an der Oberfläche zulässige Gussfehler definiert, die sich nach Lage und Grösse unterscheiden. Da derzeit die Bemessung von Stahlgussknoten für den Brückebau nicht in Normen geregelt ist, werden relativ hohe Gütestufen für die Stahlgussknoten gewählt, die mit kostenaufwändigen zerstörungsfreien Untersuchungen wie Ultraschall- oder Röntgenprüfungen nachgewiesen werden müssen. Mit der Wahl einer hohen Gütestufe wird die Ermüdungsbemessung jedoch nicht abgedeckt, da zwischen Gütestufe und Ermüdungsfestigkeit keine anerkannte Relation gegeben ist. Aufgabe ist es daher nun, in Abhängigkeit von der erforderlichen Ermüdungsfestigkeit eines Stahlgussknotens die maximal zulässigen Anfangsgrössen von Gussfehlern zu definieren.

\section{$1.3 \quad$ ZIELSETZUNG}

Die vorliegende Arbeit verfolgt folgende Ziele:

1. Quantitative Erfassung des globalen Ermüdungsverhaltens von Stahlgussknoten in einem Fachwerkträger anhand experimenteller Untersuchungen an grossmassstäblichen Probekörpern.

2. Ermittlung der für eine geforderte Ermüdungsfestigkeit maximal zulässigen Anfangsgrössen der Gussfehler in den einzelnen Bereichen eines Stahlgussknotens einer typischen Fachwerkbrücke aus Hohlprofilen in Abstimmung mit der Ermüdungsfestigkeit der Anschlussschweissnähte.

3. Entwicklung eines allgemein anwendbaren Bemessungsvorschlags für Stahlgussknoten unter Ermüdung und Bestimmung der wesentlichen Parameter.

\subsection{VORGEHENSWEISE}

Die Vorgehensweise wird schematisch in Fig. 1.5 dargestellt. Zunächst werden sowohl aus dem Offshore-Bereich als auch aus dem Stahlhochbau die bestehenden Kenntnisse über den Einsatz von Stahlgusskomponenten als Fachwerkknoten unter Ermüdungsbelastung aufgearbeitet. Die Besonderheiten des Werkstoffs Stahlguss werden für ein besseres Verständnis näher erläutert. Die Bemessungsansätze für die Ermüdungsfestigkeit von Stahlgusskomponenten und deren Schweissnahtanschlüsse werden zusammengefasst und wichtige Folgerungen für den Inhalt der vorliegenden Arbeit abgeleitet.

Anhand experimenteller Untersuchungen soll zunächst das globale Ermüdungsverhalten von Stahlgussknoten in einem Fachwerkträger quantitativ erfasst werden. In Abhängigkeit verschiedener Parameter wird untersucht, inwiefern das globale Ermüdungsverhalten von Stahlgussknoten durch die zwei Komponenten ,Beanspruchbarkeit des Stahlgussknotens' und ,Beanspruchbarkeit der Schweissnahtanschlüsse' bestimmt wird. Die Untersuchungen sollen über die in den Stahlgussknoten vorhandenen Gussfehler, die Materialeigenschaften des verwendeten Stahlgusses, den Einfluss der Schweissnahtausbildung auf die Ermüdungsfestigkeit sowie über die Ermüdungsrissstellen und das Rissfortschrittsverhalten Aufschluss geben.

Eine wirtschaftlich optimale Bemessung eines Stahlgussknotens ist dann gegeben, wenn der Ermüdungswiderstand des Gussknotens demjenigen der Anschlussnähte entspricht. Die Versuchsergeb- 
nisse zeigen eindeutig, dass die Schweissnahtanschlüsse für die globale Ermüdungsfestigkeit massgebend sind. Ihr Ermüdungsverhalten wird daher in einem parallel laufenden Forschungsprojekt detailliert untersucht. Die vorliegende Arbeit konzentriert sich auf die Abstimmung der Ermüdungsfestigkeit der Stahlgussknoten auf diejenige der Anschlussschweissnähte. Es sollen also die maximal zulässigen Anfangsgrössen der Gussfehler unter Berücksichtigung der Ermüdungsfestigkeit der Anschlussschweissnähte bestimmt werden. Dazu wird ein numerisches Modell mit Randelementen entworfen, mit dem für im Knoten eingefügte Gussfehler Spannungsintensitätsfaktoren an der Rissfront berechnet werden und das Rissfortschrittsverhalten simuliert wird. Um die Anwendbarkeit des Modells zu überprüfen, werden die berechneten Spannungen im Modell mit den experimentellen Resultaten verglichen und der Rissfortschritt aus den Versuchen plausibel simuliert.

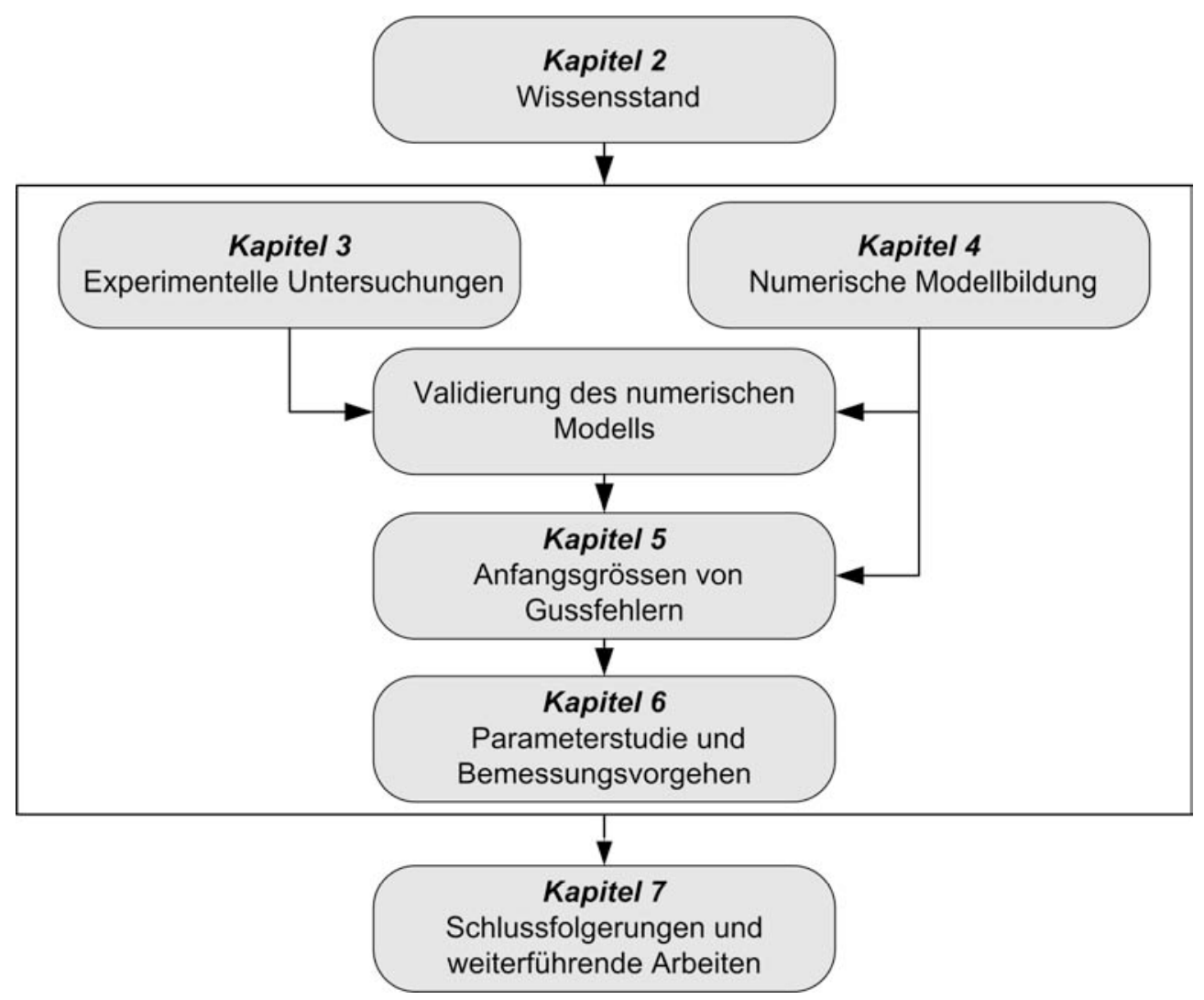

Fig. 1.5: Schematische Darstellung der Vorgehensweise

Mit Hilfe dieses Modells werden zunächst die für eine geforderte Ermüdungsfestigkeit maximal zulässigen Anfangsgrössen der Gussfehler in Stahlgussknoten, die in den Längsträgern einer typischen Stahl-Beton-Verbundbrücke eingebunden sind, berechnet. Mit dem Ziel einer allgemeinen Anwendung der Ergebnisse für Stahl-Beton-Verbundbrücken werden diese Anfangsgrössen durch eine Näherungsformel umschrieben. Dank der Näherungsformel vereinfacht sich das anhand dieser typischen Rohrfachwerkbrücke dargestellte Vorgehen für die Ermüdungsbemessung von Stahlgussknoten wesentlich. Diese Näherung dient anschliessend zur Durchführung einer Parameterstudie, die den Einfluss von Materialeigenschaften wie Bruchzähigkeit und Fliessgrenze, von verschiedenen Belastungskombinationen aus Ermüdungs- und Verkehrslast und der durch den Brückentyp bedingten Knotengeometrie auf die maximal zulässige Anfangsgrösse von Gussfehlern untersucht. 


\subsection{AUFBAU DER ARBEIT}

Nachfolgend werden Zielsetzung und Inhalt der einzelnen Kapitel zusammengefasst.

Kapitel 2 beschreibt die Entwicklung der Stahlgussknoten in Offshore-Konstruktionen, fasst die bereits bestehenden Verfahren zur Ermüdungsbemessung von Stahlgussknoten für den Einsatz in Offshore-Konstruktionen und im Stahlhoch- und Brückenbau zusammen und stellt Angaben aus verschiedenen Normenwerken zum Ermüdungsverhalten der Anschlussschweissnähte zusammen.

Kapitel 3 fasst die experimentellen Untersuchungen an grossmassstäblichen Probekörpern mit Stahlgussknoten zusammen. Es beschreibt die Fabrikation der Probekörper, den Versuchsaufbau und -ablauf sowie die Versuchsdurchführung unter statischer Last und Ermüdungslast. Die Auswertung der Rissfortschrittsdaten zeigt auf, welche der beiden Komponenten - Beanspruchbarkeit des Stahlgussknotens oder der Schweissnahtanschlüsse - massgebend für das globale Ermüdungsverhalten von Stahlgussknoten ist. Die Spannungsmessungen unter statischer Last und die Rissfortschrittsdaten dienen im Kapitel 4 zur Validierung des numerischen Modells. Die Ergebnisse der Untersuchungen über in den Stahlgussknoten vorhandene Gussfehler und Materialeigenschaften des verwendeten Stahlgusses werden für die Berechnungen in den Kapiteln 5 und 6 herangezogen.

Kapitel 4 umfasst die Modellbildung des Stahlgussknotens mit Hilfe von Randelementen (Boundary element, BE). Mit dem numerischen Modell können Spannungsintensitätsfaktoren an der Rissfront berechnet und das Rissfortschrittsverhalten simuliert werden. Es wird auf die Vorteile der Modellierung mit Randelementen für die Risswachstumssimulation eingegangen und das Prinzip der Randelementmethode erklärt. Zur Validierung des BE-Modells werden Spannungsverlauf und Rissfortschritt mit den experimentell bestimmten Daten verglichen.

Kapitel 5 erklärt das generelle Vorgehen zur Bestimmung der für eine geforderte Ermüdungsfestigkeit maximal zulässigen Anfangsgrössen von Gussfehlern in Stahlgussknoten. Die Ergebnisse beziehen sich in diesem Kapitel nur auf den Anwendungsfall einer typischen Stahl-BetonVerbundbrücke aus Rundhohlprofilen. Die Berechnung der Spannungsintensitätsfaktoren und der Differenz derselben an der Rissfront der verschiedenen Fehlerstellen unter Ermüdungs- und Verkehrslast wird erklärt. Zudem wird diskutiert, inwiefern diese Faktoren in die Rückrechnung auf die maximal zulässige Anfangsfehlergrössen eingehen.

Kapitel 6 erläutert die Verallgemeinerung der Ergebnisse aus dem Kapitel 5. Eine Näherungsformel zur Berechnung der Spannungsintensitätsfaktoren an den verschiedenen Fehlerstellen im Gussknoten wird vorgeschlagen. Dank der Näherungsformel vereinfacht sich das anhand einer typischen Rohrfachwerkbrücke dargestellte Vorgehen für die Ermüdungsbemessung von Stahlgussknoten wesentlich. Diese Näherung dient anschliessend einer Parameterstudie, die den Einfluss von Materialeigenschaften wie Bruchzähigkeit und Fliessgrenze, von verschiedenen Kombinationen aus Ermüdungs- und Verkehrslast und von der durch den Brückentyp bedingten Knotengeometrie auf die für eine geforderte Ermüdungsfestigkeit maximal zulässige Anfangsfehlergrösse untersucht. Die Parameter mit massgebendem Einfluss können so identifiziert und wichtige Folgerungen für die Festlegung der maximal zulässigen Anfangsgrösse von Gussfehlern abgeleitet werden.

Kapitel 7 fasst die wichtigsten Folgerungen der Arbeit zusammen und gibt Anregungen für weitere Arbeiten. 



\section{WISSENSSTAND ZUR ANWENDUNG VON STAHLGUSSKNOTEN}

\subsection{STAHLGUSSKNOTEN ALS KONSTRUKTIONSDETAIL - ENTWICKLUNG}

\subsubsection{Offshore-Bauwerke}

Beim Entwurf von Ölplattformen, deren Bohrturm als räumliches Fachwerk aus Stahlhohlprofilen ausgebildet ist, wurde schon früh erkannt, dass die geschweissten Knoten auf Grund ihrer niedrigen Ermüdungsfestigkeit die Schwachpunkte der Konstruktion waren. Die Kombination aus sehr hohen Spannungskonzentrationen am Schweissnahtfuss der Querstreben, Schweissnahtkerben, Schweisseigenspannungen und der zusätzlichen Beanspruchung der Hohlprofile durch Sekundärbiegemomente infolge exzentrischer Anschlüsse führt zu einer niedrigen Ermüdungsfestigkeit der Knoten. Zudem sind die Schweissnähte oftmals - insbesondere bei Knoten mit mehreren zusammenlaufenden Hohlprofilen - wegen schlechtem Zugang schwierig auszuführen, zu prüfen und zu überwachen. Das in Fig. 2.1 dargestellte Beispiel verdeutlicht den komplexen Aufbau eines geschweissten Knotens einer Ölplattform. Aus diesen Gründen hat die Offshore-Industrie als Alternative zum geschweissten Knoten den Stahlgussknoten eingeführt.

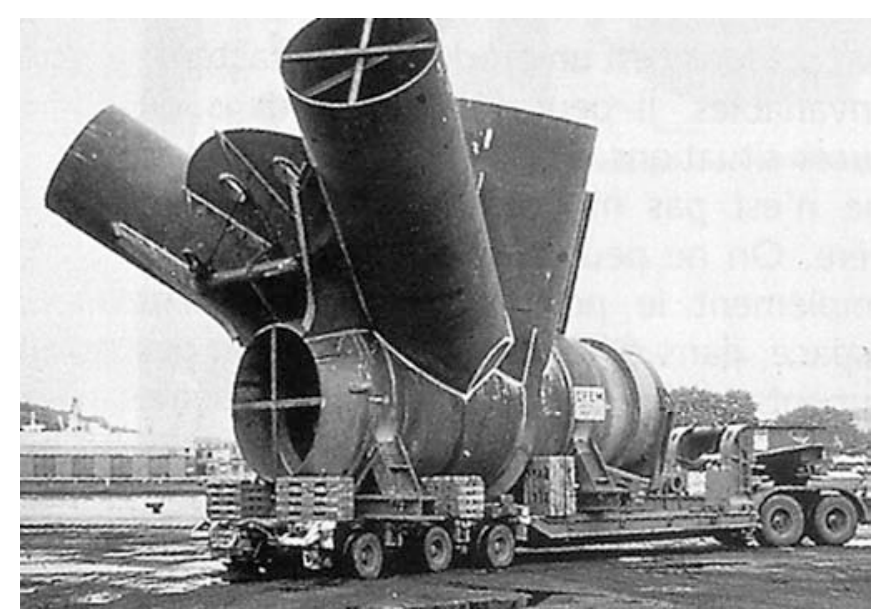

Fig. 2.1 : Beispiel eines komplexen geschweissten Rohrknotens

Bei der Herstellung von Stahlgussknoten wird flüssiger Stahl in eine Form gegossen. Ein klassisches Gussverfahren stellt dabei der Sandguss mit verlorener Form dar. Für den Stahlgussknoten wird ein in zwei Hälften aufgeteiltes Holzmodell (Fig. 2.2 a) hergestellt, das unter hohem Druck in den in einen Kasten gefüllten Formsand, oftmals ein mit Bindestoffen aus Ton und Harzen gebundener Quarzsand, abgedrückt wird. Die beiden Kästen mit den negativen Abdrücken der Knotenhälften werden anschliessend zusammengefügt, um den gesamten Hohlraum der Knotenform zu bilden.

Die Verwendung von Stahlguss ermöglicht eine dem Kraftfluss angepasste Knotenform, indem die Anschlusswinkel der Hohlprofile ausgerundet und die Wanddicken in Bereichen von Spannungskonzentrationen verstärkt werden können. Ausserdem liegen die Anschlussschweissnähte dank den Stutzen ausserhalb der Bereiche mit Spannungskonzentrationen und können leichter ausgeführt und inspiziert werden. 
Dank zunehmender Forschung auf dem Gebiet der Metallurgie und der Formtechnik wird Stahlguss seit mehr als zwei Jahrzehnten erfolgreich für Ölplattformen eingesetzt. Bereits Anfang der Achtzigerjahre wurden die Vorteile der Stahlgussknoten gegenüber geschweissten Knoten erkannt [Webster et al. 1980]. Fortschritte in der Giesstechnik führten seither zu einer stetigen Verbesserung der Schweissbarkeit des Stahlgusses [Haneke et al. 1982] [Ellis et al. 1983] [Koivula et al. 1989], der Werkstoffeigenschaften [Wood et al. 1982] [Christianus 1998] und der Zähigkeit [Ohba et al. 1979] [Richardson 1996]. Die Sicherheit hochbeanspruchter Stahlgussbauteile wird in [Gysel et al. 1984] [Gysel et al. 1984.1] in Bezug auf die Herstellung, das Prüfwesen und die Qualitätssicherung diskutiert.

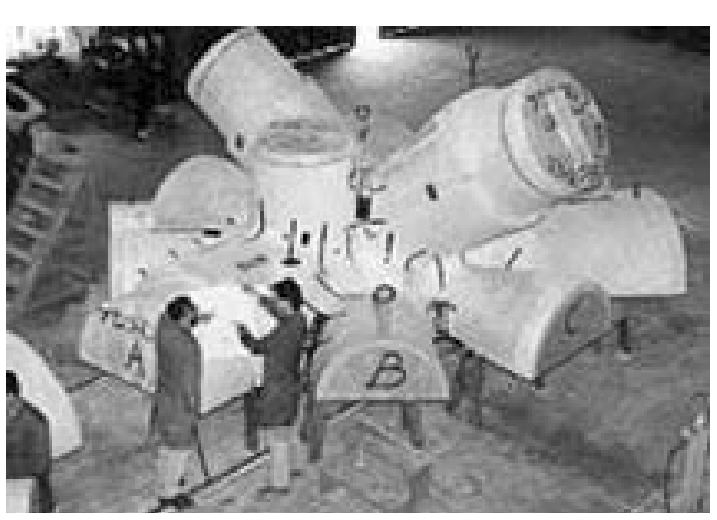

a) Holzmodell eines halben Knotens

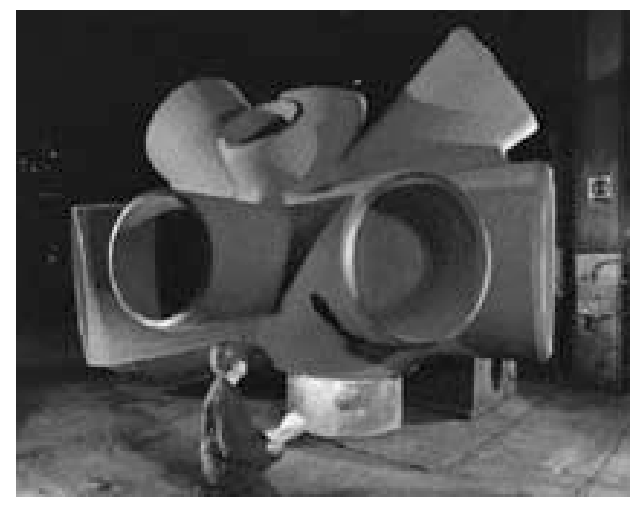

b) Stahlgussknoten bei der Abnahme

Fig. 2.2: Stahlgussknoten mit optimierter Knotenform, Beispiele [http://www.sfel.com]

Zur Simulation von Formfüllung, Erstarrung und Abkühlung steht heute spezifische Software zur Verfügung. Auch die Berechnung der Gusseigenspannungen und des zu erwartenden Verzugs ist damit effizient durchführbar. Anschnitt- und Speisertechnik ${ }^{1}$ sowie die Formauslegung lassen sich mit solcher Software verbessern, wodurch die Kosten für Nacharbeit und Ausschuss erheblich reduziert werden können.

Die Offshore-Industrie entwickelte bis heute drei Arten von Stahlgussknoten. Die klassische Lösung des Ersatzstahlgussknotens [Wood et al. 1982] empfiehlt, für die Form des Stahlgussknotens diejenige des geschweissten Knotens zu übernehmen und lediglich die exzentrischen Anschlüsse zu eliminieren sowie Ausrundungen und Wanddickenverstärkungen hinzuzufügen. Die freie Formgestaltung durch Stahlguss ermöglicht jedoch eine günstigere Knotenform (Fig. 2.2 b) mit kegelförmigen Stutzen, die kompaktere Knoten erlaubt [UEG 1985]. Um Schweissarbeiten einzusparen werden auch Stahlgussverbundknoten angefertigt [Sonsino et al. 1988] [Christianus 1998]. Dabei handelt es sich beim Beispiel eines K-Knotens um einen Einsatz mit angeordneten Stutzen, der in den Gurt eingeschweisst wird. Die Stahlgusskomponente ersetzt so lediglich die Anschweisszonen der Diagonalen.

\footnotetext{
${ }^{1}$ Die Anschnitttechnik beinhaltet alle Massnahmen, die mit einem durchdacht gestalteten und dimensionierten Anschnittsystem beim Giessen in Zusammenhang stehen. Die Gestaltung ist insofern wichtig, da für die Gussqualität eine fachgerechte Formfüllung, gesteuerte Erstarrung und Nachspeisung gewährleistet sein müssen. Die Anschnitttechnik hat ihren Schwerpunkt in der Formfüllung, während die Speisertechnik für das Gleichgewicht des Volumens bei der Erstarrung sorgt.
} 


\subsubsection{Stahlhoch- und Brückenbau}

Im Stahlhochbau werden Stahlgusskomponenten seit dem Aufkommen von Tragwerkskonstruktionen aus Stahlhohlprofilen als Verbindungsmittel zwischen den Hohlprofilen eingesetzt. In [Schlaich et al. 1999] [Schlaich et al. 1999.1] und [Schober 2000] findet sich eine gute Übersicht über die Verwendung von Stahlgussknoten in Seilkonstruktionen, Dachtragwerken, Fussgängerbrücken sowie Strassen- und Bahnbrücken.

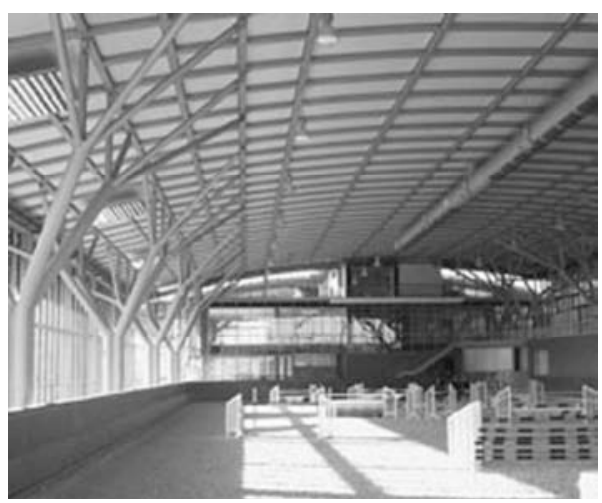

a) Reithalle bei Bulle (CH), 2002 [http://www.mueller-offenburg.de]

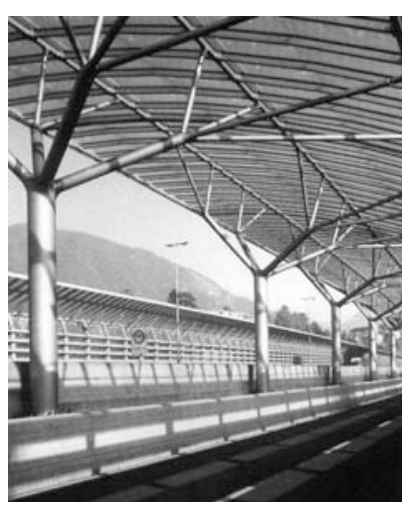

b) Lärmschutzwand bei Chiasso (CH), 2003 [SZS 2005]

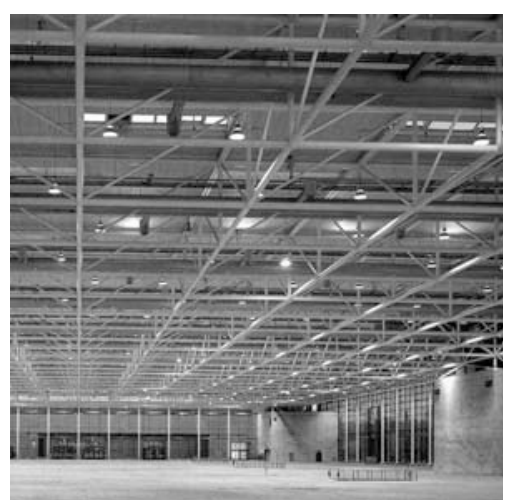

c) Halle 13 der Deutschen Messe AG Hannover (D), 1999

[Ackermann 1999]

Fig. 2.3: Beispiele für Gabelstützen mit Stahlgussknoten im Stahlhochbau

Die Dachtragwerke zeichnen sich durch weitgreifende Gabelstützen aus, auf denen die Dachkonstruktion abgestützt wird. Bekannte Beispiele sind die Reithalle bei Bulle (CH, 2002) in Fig. 2.3 a), die Terminals 1 und 3 des Flughafens Stuttgart (D, 1989 / 2004, [http://www.gmp-architekten.de/]) und die neue Lärmschutzwand auf der Autobahn A2 bei Chiasso (CH, 2003) in Fig. 2.3 b). Stahlgussknoten finden aber auch in klassischeren Tragwerken wie räumlichen Fachwerkkonstruktionen Anwendung, wie das Beispiel der Halle 13 der Deutschen Messe AG in Hannover in Fig. 2.3 c) zeigt. Im Zuge der Sanierung und Modernisierung des Berliner Olympiastadions im Jahre 2000 bis 2004 wurde die Dachkonstruktion mit Fachwerkträgern aus Rundhohlprofilen und Stahlgussknoten ausgebildet.

Das Konzept der Gabelstützen wurde nicht nur bei Fussgängerbrücken wie derjenigen in Sindelfingen (Fig. 2.4 a) oder der Fussgängerbrücke am Pragsattel II in Stuttgart (Fig. 2.4 b), sondern auch bei Strassenbrücken erfolgreich übernommen. Bei der 1997 fertig gestellten Strassenüberführung über die A8 in der Nähe von Leonberg (Fig. 2.4 c) wurde ebenfalls die bereits beschriebene Baumstruktur als tragendes Element eingesetzt. Auch hier wurden die Gabeln aus Stahlguss gefertigt und mit den anlaufenden Hohlprofilen verschweisst. Die Fahrbahnplatte liegt im Verbund auf gegossenen Kopfplatten auf.

Die Humboldthafenbrücken im Bereich des Lehrter Bahnhofs in Berlin (Fig. 1.3 auf Seite 2) sind die ersten Eisenbahnbrücken, bei denen Stahlgusskomponenten in sicherheitsrelevanten Funktionen unter statischen Lasten und Ermüdungsbeanspruchung eingesetzt wurden [Seifried et al. 1999]. Darüber hinaus wurden diese Stahlgusskomponenten mit Hohlprofilen verschweisst. Beides stellte eine Neuigkeit für Bauten der Deutschen Bahn AG dar. Daher und auf Grund des hohen Sicher- 
heitsbedürfnisses im öffentlichen Personenverkehr wurden umfangreiche Untersuchungen an den für diese Brücken eingesetzten Gussteilen und den Schweissverbindungen Stahlguss-Stahl veranlasst [Mang et al. 1999] [Schlaich et al. 1999.2]. Die Baugenehmigung für die Humboldthafenbrücken wurde erst nach Durchführung dieser zeit- und kostenintensiven Untersuchungen erteilt.

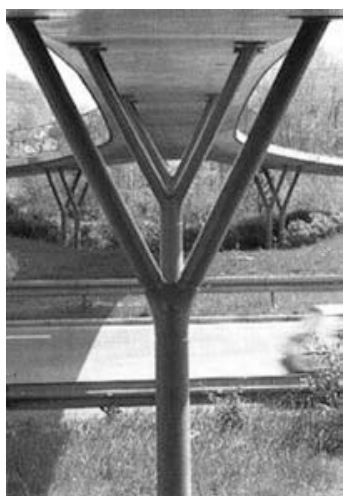

a) Fussgängerbrücke,

Sindelfingen (D), 1986

[http://www.archin-

form.net]

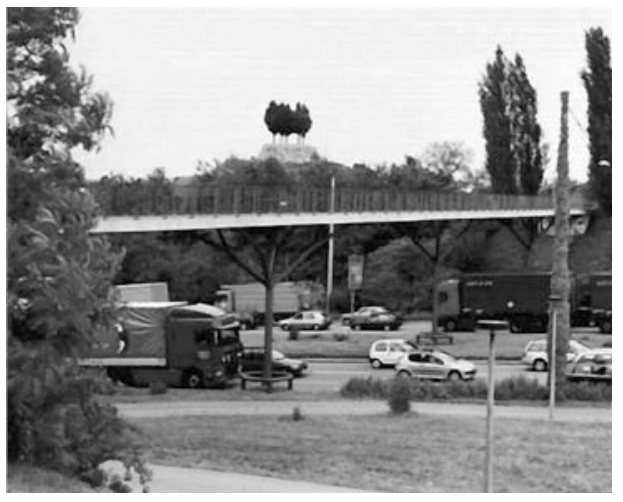

b) Fussgängerbrücke am Pragsattel II,

Stuttgart (D), 1992

[http://www.structurae.de]

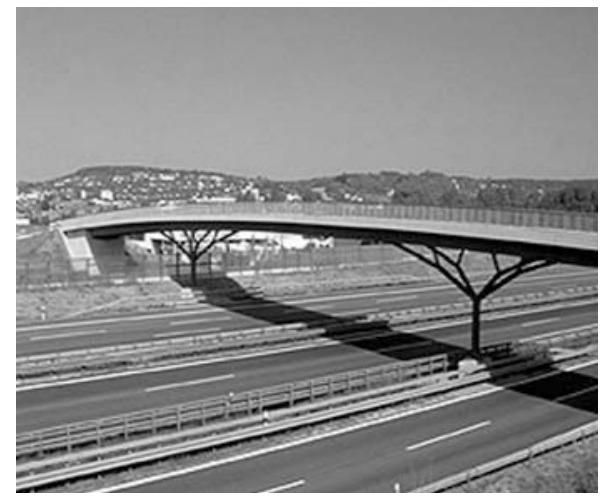

c) Feldwegbrücke über Autobahn A8, Leonberg (D), 1997

[http://www.sbp.de]

Fig. 2.4: Beispiele für Gabelstützen mit Stahlgussknoten für Fussgängerbrücken

Ein kürzlich fertig gestelltes Beispiel einer Strassenbrücke ist die Nesenbachtalbrücke bei Stuttgart (Fig. 1.1 auf Seite 1). Bei dieser Fachwerkbrücke wurden alle Knoten aus Stahlguss hergestellt [Schlaich et al. 2000.1]. Da die Brücke in einer Kurve mit variablem Radius liegt, ergeben sich für jeden der 130 Fachwerkknoten unterschiedliche Anschlusswinkel der Hohlprofile. Trotzdem konnten mit nur zwei verschiedenen Knotengeometrien sämtliche Rohranschlüsse realisiert und so die Herstellungskosten für die Stahlgussknoten optimiert werden. Bei diesem Bauwerk konnte teilweise auf die beim Bau der Humboldthafenbrücken gewonnene Erfahrung zurückgegriffen werden. Auch hier erwiesen sich die fehlenden Richtlinien für die ermüdungsbeanspruchten Stahlgusskomponenten als ein Hindernis, das nur mit zusätzlichem Aufwand überwunden werden konnte.

Die Bahnbrücke Oosterbeek bei Aanbrug (NL) hat einen offenen Querschnitt mit zwei ebenen, mit Querstreben verbundenen Fachwerklängsträgern (siehe Fig. 1.2 auf Seite 1). Sie trägt über mehrere Felder mit einer Spannweite von 56 m. Die Knoten im Fachwerkträger sind als K-Knoten mit einem zusätzlichen Knotenstutzen für die Querstrebe ausgebildet.

Im Zuge der neuen Umgehungsstrasse Korntal-Münchingen (D) wurde eine $300 \mathrm{~m}$ lange Talbrücke erforderlich [Bernhardt et al. 2003]. Das Haupttragwerk besteht aus einem räumlichen Fachwerk mit einem Untergurt, wobei die Fahrbahnplatte durch Verbund die Rolle der Obergurte übernimmt. Auch in diesem Beispiel sind die Knotenpunkte der Fachwerkstäbe als Stahlgussknoten ausgebildet, um die Spannungskonzentrationen im Knoten möglichst niedrig zu halten und die Schweissnähte aus den Bereichen hoher Spannungskonzentrationen in weniger beanspruchte Zonen zu legen. Die Brücke wurde im März 2005 eingeweiht.

Die Talbrücke Sankt Kilian auf der Autobahn A73 (D) ist derzeit im Bau und wird voraussichtlich 2006 fertig gestellt sein. Die zwei Längshauptträger sind als Fachwerkträger mit Rundhohlprofilen 
ausgebildet und haben ein gleichschenkliges Dreieck als Querschnitt. Die Fahrbahnplatte übernimmt die Rolle der Obergurte. Die Brücke hat acht Felder mit einer Länge von $39.3 \mathrm{~m}$ bis $61.5 \mathrm{~m}$, eine Gesamtlänge von knapp $450 \mathrm{~m}$ und wird über Gabelstützen getragen. Auch hier wurden für die Fachwerkknoten Stahlgussknoten gewählt.

Bei den genannten Beispielen wurde für die Stahlgussknoten der Werkstoff GS-20 Mn $5 \mathrm{~V}$ nach [DIN 17182:1992] und im Falle der Oosterbeek Brücke der Stahlguss G10 MnMo V 6-3 nach [DIN 17205-1: 1998] verwendet. Für die Anschweissenden der Knotenstutzen wurde generell die Gütestufe 1 und für den Restbereich des Knotens die Gütestufe 2 und/oder 3 nach [DIN 1690-2:1985] gewählt.

\subsection{DER WERKSTOFF STAHLGUSS}

\subsubsection{Gussfehler}

Beim Giessen von Gussstücken können typische Gussfehler wie Lunker, Gasblasen, Sand-, Schlacken- und Schaumstellen, Warm- und Kaltrisse, Seigerungen und nicht-metallische Einschlüsse auftreten. In [Knipp 1961], [Beeley 2001] und im Gussfehleratlas [IfG - DK 2005] werden Entstehung und Ursachen der Gussfehler ausführlich beschrieben. Nachfolgend werden die Kernpunkte zu den vier wichtigsten Fehlerfamilien, die auch in den Liefernormen für Stahlgusskomponenten (Abschnitt 2.2.3) aufgeführt sind, kurz zusammengefasst.

\section{Gasblasen}

Gasblasen, genauer Gashohlräume, können aus Gasen entstehen, die als Reaktionsgase, durch gelöste Gase, durch mitgerissene Luft beim Giessen und aus dem Formsand in das flüssige Metall gelangt sind. Die Gasblasen sind Hohlräume mit meist glatten Wänden, blank oder oxidiert. Bei grossen Blasen können die Wände auch gewellt sein. Sie haben stets eine rundliche Form und können auch grössere Ausdehnungen (dann stets flache Hohlräume) bis zu mehreren Millimetern Durchmesser haben. In Fig. 2.5 ist ein Beispiel einer Stahlgusskomponente mit Gasblasen abgebildet.

Die Reaktionsgase entstehen aus der Reaktion von Eisenoxyd ( $\mathrm{FeO}$ ) mit Kohlenstoff $(\mathrm{C})$ oder mit Wasserstoff $\left(\mathrm{H}_{2}\right)$. Normalerweise treten diese Reaktionen während des Schmelzvorgangs auf. Treten sie während des Giessvorgangs auf, ist der flüssige Stahl entweder noch nicht genügend desoxydiert oder die Temperatur ist noch zu hoch. Solange die Viskosität des Stahls sehr tief ist, können die Reaktionsgase entweichen, ist dies nicht mehr der Fall, werden sie im Metallgefüge eingeschlossen. Je grösser das Erstarrungsintervall eines Metalls ist, desto mehr neigt es zu Gasporositäten, denn in dieser Abkühlphase steigt die Viskosität und die sich bildenden Gasblasen werden zunehmend am Austreten aus der Schmelze gehindert. Da für eutektische Legierungen oder reine Metalle die Abkühlphase sehr viel kürzer ist, sind sie weniger anfällig auf Gasblasen.

Im flüssigen Stahl gelöste Gase wie Sauerstoff, Stickstoff oder Wasserstoff werden durch die abnehmende Löslichkeit mit sinkender Temperatur wieder freigegeben. In der Erstarrungsphase nimmt die Löslichkeit sprunghaft ab. Die Entwicklung von Gasblasen aus gelösten Gasen kann verhindert werden, indem beim Schmelzvorgang der Kontakt dieser Gase mit der Schmelze eingeschränkt, die Löslichkeit der Gase in der Schmelze durch Zugabe von Stoffen herabgesetzt oder verhindert oder das Entweichen der frei werdenden Gase aus der Schmelze ermöglicht wird. Eine Quelle für gelösten Wasserstoff ist bei allen Giessmetallen Wasser. Die wichtigste Massnahme zur 
Vermeidung von Gasblasen durch gelöste Gase ist daher das Fernhalten von Feuchtigkeit von der Schmelze.

Stahlgusskomponenten werden in Gussformen aus Formsand gegossen. Durch das Aufwärmen der Form beim Giessen entwickeln sich im Formstoff aus Feuchtigkeit, gebundenem Wasser und den organischen Stoffen, die dem Formsand als Bind- und Auflockerungsmittel zugesetzt wurden, Gase. Beim Entweichen nehmen diese den Weg des geringsten Widerstands und dringen, wenn der Formsand nicht gasdurchlässig genug ist, in den flüssigen Stahl ein. Wie bereits erklärt wurde, können die Gasblasen nicht mehr aus dem flüssigen Stahl entweichen und werden eingeschlossen. Je mehr Gasträger der Formsand aus Gründen der Formbarkeit und Festigkeit erfordert, desto gasdurchlässiger sollte er sein.

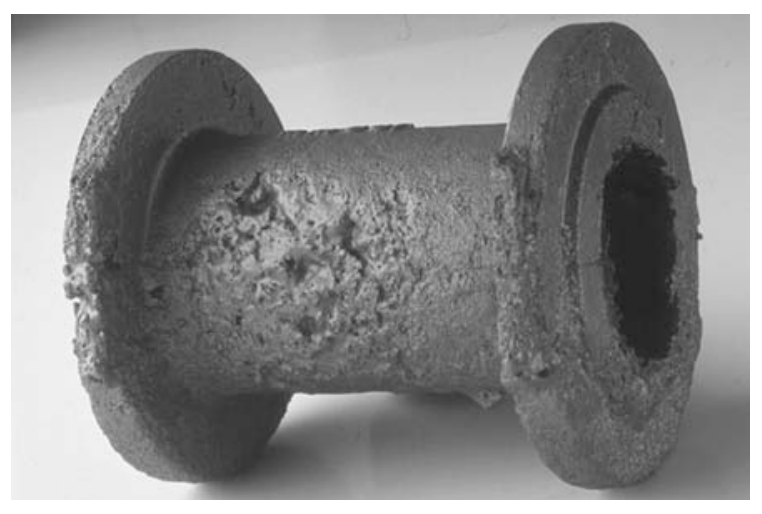

Fig. 2.5: Beispiel für Gasblasen [IfG - DK 2005]

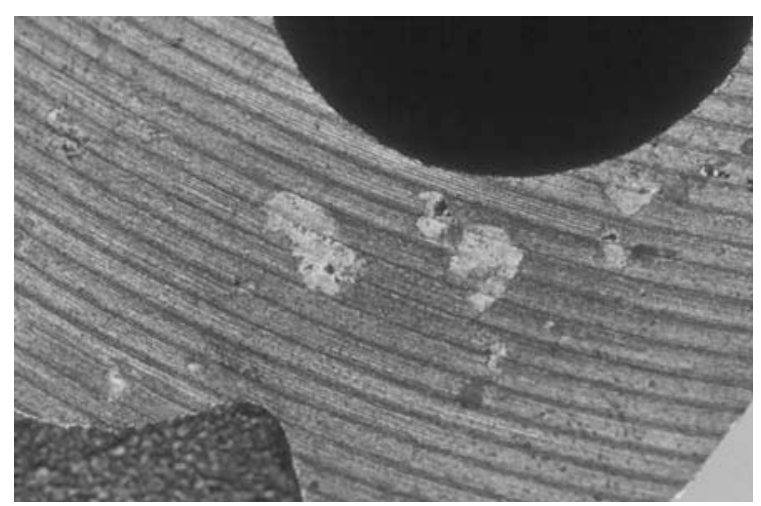

Fig. 2.6: Beispiel für Schlackeneinschlüsse [IfG DK 2005]

\section{Nichtmetallische Einschlüsse}

$\mathrm{Zu}$ den nichtmetallischen Einschlüssen gehören unter anderen eingeschlossene Schlacketeilchen (siehe Beispiel in Fig. 2.6) und Oxyde sowie Sulfide und Phosphide infolge von Seigerungsvorgängen, die durch den Schmelzprozess in das Giessmetall gelangen. Urheber für die Oxyde ist die Sauerstoffverbindung FeO, die durch oxydische Zusätze oder durch die Oxydation des Eisens beim Frischen in die Schmelze gelangt. FeO ist bestimmend für die Menge der Einschlüsse, denn es reagiert mit Desoxydationsstoffen wie Mangan, Silizium und Aluminium zu $\mathrm{MnO}, \mathrm{SiO}_{2}$ und $\mathrm{AlO}_{3}$ oder zu Silikaten $\left(\left(\mathrm{SiO}_{4}\right)^{-4}\right)$, die zum Teil in der Schmelze zurückgehalten werden.

\section{Lunker}

Lunker sind fast immer erst bei Bearbeitung erkennbar. Die Zonen sind sichtbar „lockeren“ Gefüges, zuweilen von schwammartiger Struktur oder eine Ansammlung von vielen kleinen Poren (siehe Beispiel in Fig. 2.7).

Da Stahl im flüssigen Zustand ein grösseres spezifisches Volumen aufweist als im festen Zustand, füllt der in eine Form gegossene Stahl im festen Zustand den Formhohlraum nicht mehr ganz aus. Beim Abkühlen gibt der Stahl seine Wärme fortschreitend von aussen nach innen an die Formwände oder die Aussenluft ab, wobei die Abkühlgeschwindigkeit in den äusseren Schichten am grössten ist. Die bereits erstarrte Aussenschicht der gegossenen Form schliesst nun noch flüssigen Stahl ein, dessen Volumen beim weiteren Abkühlen schrumpft. Da die äussere Schicht bereits erstarrt ist, kommt es zwangsläufig zur Bildung eines Hohlkörpers im Inneren des Gussstücks. Dieser 
Lunker oder auch Schwindungslunker genannte Hohlraum befindet sich entsprechend an der Stelle, wo der Stahl zuletzt erstarrt ist. Je langsamer die Abkühlung des Gussstücks verläuft - also je kleiner der Temperaturunterschied zwischen der äusseren und der inneren Schicht ist - desto kleiner ist die Gefahr eines Lunkers im Inneren. Der Volumenverlust kann jedoch nicht kompensiert werden. Alternativ zur langsamen Abkühlung kann ein Temperaturunterschied auch ausgeglichen werden, indem die Wärme mit Hilfe von Kühlkörpern schnell aus dem Inneren des Gussteils evakuiert wird. Eine weitere Möglichkeit besteht darin, je nach Querschnitt des Gussstücks verschiedene Sorten von Formsand mit verschiedenen Wärmeleitzahlen einzusetzen. Die wirkungsvollste Massnahme zur Vermeidung von Lunkern ist das Ansetzen von Reservoiren, auch Steiger, Trichter oder Speiser genannt. Die Reservoire werden an den Stellen des Gussstücks angesetzt, wo Lunker zu erwarten sind, damit flüssiger Stahl zu den gefährdeten Stellen nachfliessen kann. Vorraussetzung für eine gelungene Speisung ist, dass das Reservoir solange flüssig bleibt, bis die Erstarrung des Gussstücks abgeschlossen ist.

Die Gestaltung der Form des Gussstücks ist ebenfalls ein wichtiger Aspekt zur Vermeidung von Lunkern. Es muss darauf geachtet werden, dass bei der Erstarrung der Zufluss von flüssigem Stahl nicht unterbrochen wird. Erstarrt zum Beispiel ein Gussstück mit einer konstanten Wanddicke, so ist die Wanddickenmitte lunkeranfällig, weil es über die gesamte Länge gleichzeitig erstarrt und kein flüssiger Stahl nachfliessen kann. Variiert die Wanddicke jedoch mit einem Winkel von ungefähr $4^{\circ}$, so erstarrt die Wanddicke in Richtung der Wanddickenzunahme, aus der auch der flüssige Stahl nachfliesst. Giesstechnische Erfahrungen haben gezeigt, dass die Wanddicke höchstens über eine dreimal der Wanddicke entsprechende Länge konstant sein darf.

\section{Risse}

Risse entstehen in Gussstücken infolge von Eigenspannungen, die auf eine ungleichmässige Schwindung des Gussstücks bei der Abkühlung zurückzuführen sind. Ursache der Ungleichmässigkeit ist die Behinderung der Schwindung an einzelnen Stellen des Gussstücks. Die Eigenspannungen sind proportional zum Elastizitätsmodul $E$, zum Temperaturausdehnungskoeffizienten $\alpha$ und zur Temperaturdifferenz $\Delta T$. Sind die Eigenspannungen im Gussstück zu hoch, kommt es zu einem Riss. Dabei wird zwischen Warm- und Kaltrissen unterschieden.

Der Warmriss unterscheidet sich vom Kaltriss durch den Ort der Entstehung und durch die Form der Rissfläche (siehe Beispiel in Fig. 2.8). Trifft in einem Gussstück ein bereits abgekühlter und erstarrter Teil, welcher unter Zugeigenspannung steht, auf eine noch flüssige Stelle, so kommt es zu einem Warmriss, da auf Grund des temperaturbedingt noch niedrigen Elastizitätsmoduls $E$ nur kleine Spannungen aufgenommen werden können. Dabei wird die bereits erstarrte, äussere Kruste von der noch flüssigen Stelle abgerissen, woraus sich die zackige und verästelte Bruchfläche von Warmrissen ergibt. Die Risse heissen also Warmrisse, weil sie in der Nähe von „heissen Stellen“ entstehen. Diese Stellen befinden sich bei Querschnittsübergängen, Materialanhäufungen, scharfen Ecken und in der Nähe von Speisern.

Der Kaltriss entsteht bei Temperaturen, bei denen sich der Stahl bereits vollständig im elastischen Zustand befindet. Die durch die unterschiedliche Abkühlung und damit Schwindung hervorgerufenen Eigenspannungen belasten das Gussstück, bis im kleinsten Querschnitt die Eigenspannungen die Zugfestigkeit übersteigen und es zum Bruch kommt. Die Bruchfläche ist im Gegensatz zum Warmriss glatt. 
Massnahmen zur Verhinderung von Rissen können in der Form- und Giesstechnik sowie bei der Gestaltung des Gussteils ergriffen werden. Es müssen insbesondere die Voraussetzungen für eine möglichst gleichmässige Abkühlung des Gussstücks in allen Querschnitten geschaffen und Schwindungswiderstände beseitigt werden. Die angewandten Massnahmen sind dieselben, die auch für die Verhinderung von Lunkern wirksam sind.

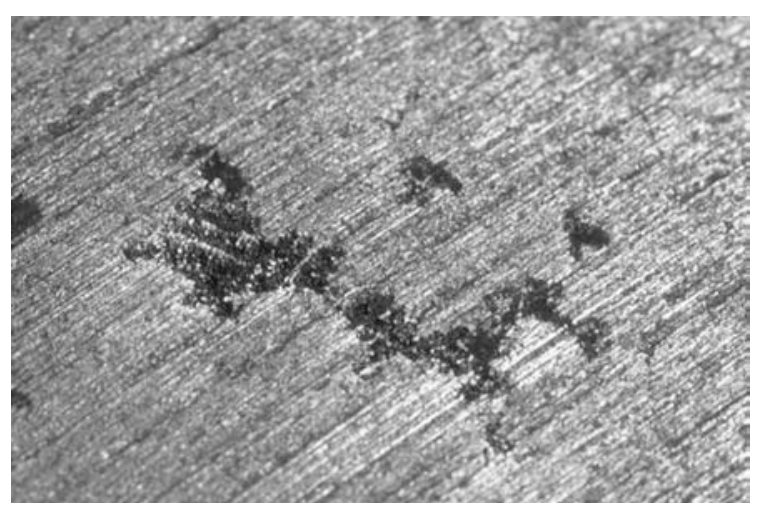

Fig. 2.7: Beispiel eines Lunkers [IfG - DK 2005]

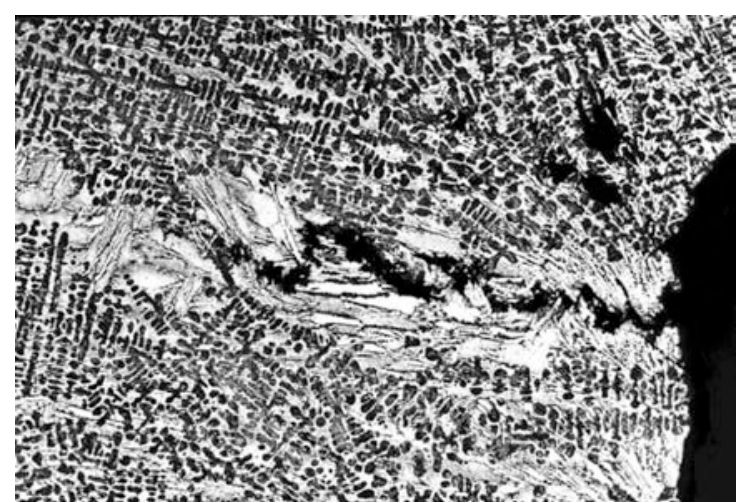

Fig. 2.8:Beispiel eines Warmrisses [IfG - DK 2005]

\subsubsection{Nachweis von Gussfehlern}

In der Liefernorm [DIN 1690-2:1985] werden die Magnetpulverprüfung, die Farbeindringprüfung, die Durchstrahlungs- und die Ultraschallprüfung als anerkannte Prüfverfahren für Gussstücke zugelassen.

Zur Oberflächenprüfung von Gussteilen aus Stahl können die Magnetpulverprüfung gemäss [StahlEisen-Prüfblatt 1935:1982] die Farbeindringprüfung gemäss [Stahl-Eisen-Prüfblatt 1936:1982] angewendet werden. Die Magnetpulverprüfung dient zum Nachweis makroskopischer Fehler an oder dicht unter der Oberfläche von ferromagnetischen Gussstücken. Bei der Farbeindringprüfung können mittels Eindringmittel Fehler wie Risse, Überlappungen, Falten, Gasblasen und Bindefehler, die zur Oberfläche hin offen sind, nachgewiesen werden. Mit diesen Methoden kann zwar das Vorhandensein von Rissen eindeutig nachgewiesen, nicht aber die Risstiefe gemessen werden. Die Fehleranzeigen sind nach Grösse und Häufigkeit zu beurteilen. Die zulässigen Grössen und Häufigkeiten sind mit dem Hersteller (meist auf der Basis der Norm [DIN 1690-2:1985]) zu vereinbaren.

Zur Überprüfung des Inneren von Gussteilen wird die Ultraschallprüfung nach [Stahl-EisenPrüfblatt 1922:1985] und die Durchstrahlungsprüfung nach [DIN 54111-2:1982] durchgeführt. Die Ultraschallprüfung erzeugt kein direktes Abbild des Fehlers, d. h. das Prüfergebnis muss interpretiert werden. Deshalb ist oft eine Vergleichsprüfung an Testkörpern mit definierten Fehlergrössen notwendig. Typische Bezugsreflektoren, deren Echos mit den Echos im Bauteil verglichen werden, sind Querbohrungen, Nuten und Flachbodenbohrungen, so genannte Kreisscheibenreflektoren. In [Stahl-Eisen-Prüfblatt 1922:1985] werden Registriergrenzen für Fehler anhand des Durchmessers des Kreisscheibenreflektors definiert. Bei der Überprüfung des Gussstücks wird die Echoanzeige des Kreisscheibenreflektors mit derjenigen des Fehlers verglichen. Alle Echoanzeigen, die die Registriergrenzen erreichen oder überschreiten, sind zu erfassen. Je nach Prüfklasse und Wanddicke des Gussstücks wird ein Kreisscheiben-Vergleichsreflektor bei Reflektoren mit/ohne messbare 
Ausdehnung von $3 \mathrm{~mm}$ bis $12 \mathrm{~mm}$ vorgeschrieben. Festlegungen zur Beurteilung von Echoanzeigen im Hinblick auf ihre Zulässigkeit sind mit dem Hersteller (meist auf der Basis der Norm [DIN 1690-2:1985]) zu vereinbaren.

Die Norm [DIN 54111-2:1982] ist für die Durchstrahlungsprüfung von Gussstücken aus Eisenwerkstoffen, insbesondere Stahlguss, anzuwenden. Durch die Norm soll erreicht werden, dass Durchstrahlungsbilder eine dem Prüfgegenstand angemessene und dem Stand der Prüftechnik entsprechende Bildqualität aufweisen. Dazu werden je nach Prüfklasse und zu durchstrahlendem Dickenbereich die Strahlenquelle und die Filmklassen vorgegeben. Bei der Auswertung von Durchstrahlungsbildern werden die Gussfehler gekennzeichnet und können gemäss den Spezifikationen in [ASTM E 446-98] [ASTM E 186-98] [ASTM E 280-98] eingeteilt werden.

\begin{tabular}{|c|c|c|c|c|c|c|c|}
\hline \multirow{3}{*}{ Prüfmethode } & \multicolumn{7}{|c|}{ Information über } \\
\hline & \multirow[t]{2}{*}{ Ort } & \multirow[t]{2}{*}{ Art } & \multirow[t]{2}{*}{ Form } & \multirow{2}{*}{$\begin{array}{l}\text { Grösse bzw. } \\
\text { Tiefe }\end{array}$} & \multirow[t]{2}{*}{ Orientierung } & \multicolumn{2}{|c|}{ Nachweisgrenze $^{2}$} \\
\hline & & & & & & Risstiefe & Risslänge \\
\hline Durchstrahlung & 2 & 1 & 1 & 2 & 2 & \multicolumn{2}{|c|}{$>0.4-2 \%$ der Wanddicke } \\
\hline Ultraschall & 1 & 2 & 2 & 2 & 3 & \multicolumn{2}{|c|}{$\begin{array}{c}\text { Risssignal }>2 \times \text { Gefügeanzeige } \\
\text { Risstiefe }>3 \text { x Oberflächenrauheit }\end{array}$} \\
\hline Magnetpulver & $1^{1}$ & 2 & 2 & 2 & 2 & $>10 \mu \mathrm{m}$ & $>100 \mu \mathrm{m}$ \\
\hline Farbeindringung & $1^{1}$ & 2 & 2 & 2 & 2 & $>20 \mu \mathrm{m}$ & $>1 \mathrm{~mm}$ \\
\hline \multicolumn{8}{|c|}{1 gut, 2 mässig, 3 schlecht } \\
\hline
\end{tabular}

Tab. 2.1 : Vergleich zerstörungsfreier Prüfverfahren für den Nachweis von Rissen (Auszug für die erwähnten Prüfmethoden) [Blumenauer 1993]

Für die bruchmechanische Bewertung von Fehlern ist eine hohe Zuverlässigkeit der angewandten Prüfmethode erforderlich. Daher wird in [Blumenauer 1993] ein relativer Vergleich der verschiedenen Prüfmethoden hinsichtlich des Nachweises von Rissen angestellt. Für einen bruchmechanischen Nachweis ist nicht die kleinste nachweisbare Ungänze, sondern die grösste Ungänze, die bei der Überprüfung übersehen werden kann, von Interesse. In Tab. 2.1 werden die Nachweisgrenzen der vier oben erwähnten Prüfmethoden gemäss [Blumenauer 1993] wiedergegeben. Die Ultraschallprüfbarkeit hängt einerseits von der Wanddicke und andererseits von der Oberflächenbeschaffenheit ab. Aus diesem Grund muss das Echo des Fehlers (Risssignal) mindestens zweimal so gross sein wie der Störpegel (Gefügeanzeige), damit der Fehler eindeutig detektierbar ist. Die Angaben für die Nachweisgrenze der Risstiefe sind nicht präzise, da nicht festgelegt ist, wie die Oberflächenrauheit zu messen ist. Es wird aber davon ausgegangen, dass mit zunehmender Oberflächenrauheit die Interpretation der Echoanzeigen schwierig wird und daher die Nachweisgrenze proportional zur Oberflächenrauheit ist. Daher wird in [Stahl-Eisen-Prüfblatt 1922:1985] vorausgesetzt, dass die Prüfflächen des Gussstücks so beschaffen sind, dass eine einwandfreie Ankopplung der Prüfköpfe möglich ist. In [Trumpold et al. 1996] und [Christianus et al. 1998] wird auf die Problematik zur Messung der Oberflächenrauheit näher eingegangen.

In Abschnitt 6.3.3.4 wird auf die Detektierbarkeit von Gussfehlern im Zusammenhang mit den gängigen Gütestufen näher darauf eingegangen. 


\subsubsection{Liefernorm für Stahlgusskomponenten}

Für die bereits in Europa erbauten Brücken mit Stahlgussknoten wurde die Qualität nach der Liefernorm [DIN 1690-2:1985] festgelegt. Diese Norm definiert fünf verschiedene Gütestufen für Stahlgusskomponenten. Für jede Gütestufe sind im Kern- und Randbereich oder an der Oberfläche des Gussteils entsprechend der Prüfmethode höchstzulässige Fehleranzeigen für die Längen-, Tiefen- und Breitenausdehnung des Gussfehlers sowie dessen Einzelfläche und grösste Gesamtfläche (bei mehreren Gussfehlern) definiert. Für die Gütestufe 1 sind nur kleinste Gussfehler zulässig. Die Gütestufe 5 lässt die grössten Fehler zu, umschreibt also die schlechteste Gussqualität.

Die Einteilung der Gussstücke nach Gütestufen erfolgt für die äussere Beschaffenheit (S) auf Grund von Magnetpuler- und Eindringprüfungen (M und E), für die innere Beschaffenheit (V) auf Grund von Durchstrahlungs- und Ultraschallprüfungen ( $\mathrm{R}$ und $\mathrm{U}$ ). Demnach setzt sich zum Beispiel die Normbezeichnung für die Gütestufe eines Gussteils, dessen Oberflächenbeschaffenheit mit Magnetpulverprüfung auf die Gütestufe 2 und dessen innere Beschaffenheit mit Ultraschallprüfung auf Gütestufe 3 hin überprüft wird, zu „Gütestufe DIN 1690 - MS2 - UV3“ zusammen.

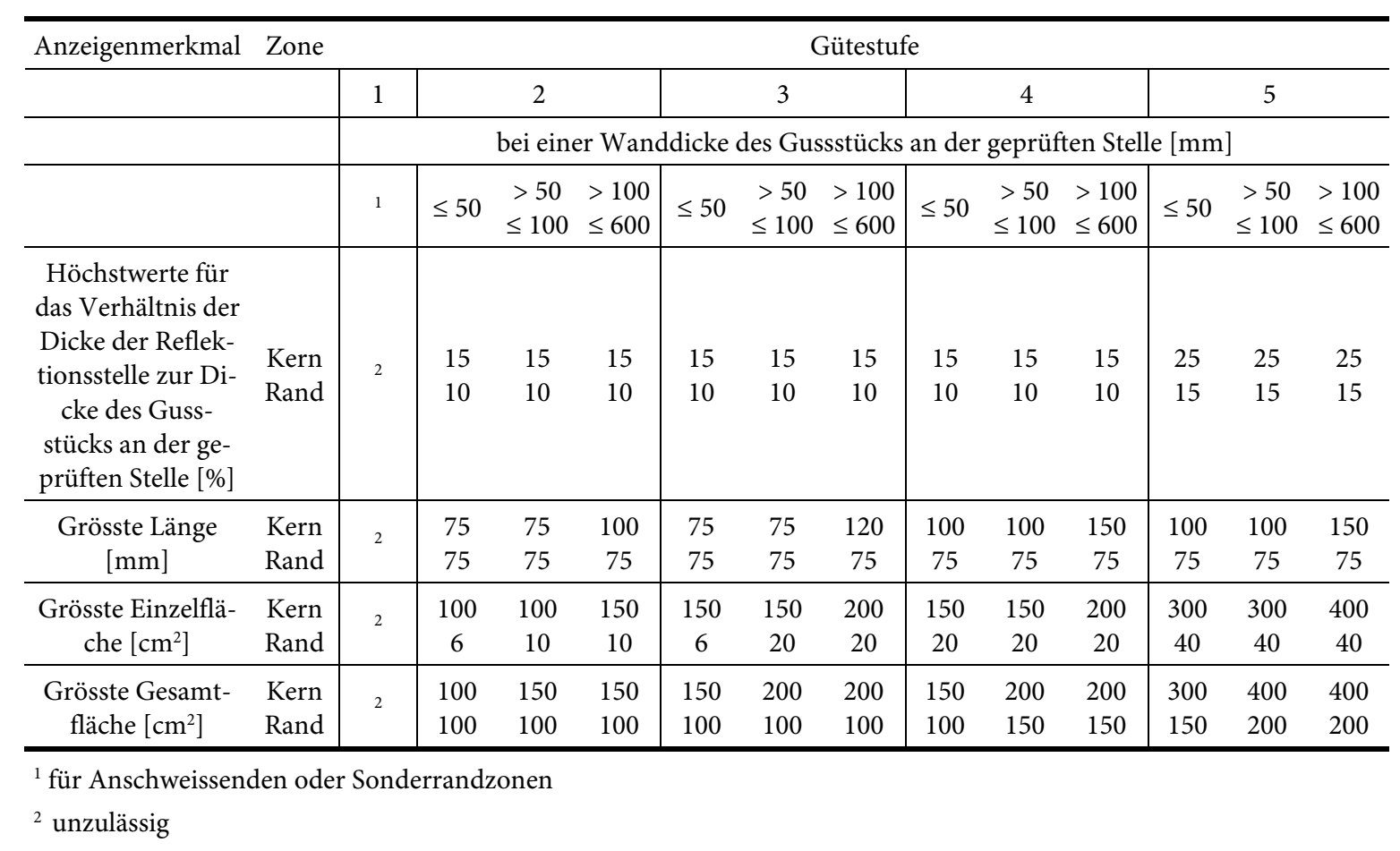

Tab. 2.2: Festlegung über höchstzulässige Anzeigenmerkmale bei der Ultraschallprüfung für Reflektoren mit messbarer Ausdehnung, Auszug aus Tabelle 3 [DIN 1690-2:1985]

In den amerikanischen Spezifikationen [ASTM E 446-98] [ASTM E 186-98] [ASTM E 280-98] sind für verschiedene Gussfehlerarten (Gasblasen, nichtmetallische Einschlüsse, Lunker, Risse) in verschiedenen Grössen (Kategorie 1 - 5) Durchstrahlungsfilme katalogisiert. Diese Durchstrahlungsfilme werden bei einer Durchstrahlungsprüfung als Vergleichsanzeigen herangezogen. In [DIN 1690-2:1985] wird angegeben, welche Durchstrahlungsfilme einer Gütestufe zugeordnet werden. Dabei werden die Gussfehler einer Grösse (Kategorie) ohne Berücksichtigung der Fehlerart einer Gütestufe zugeordnet. Die Gütestufen 1 - 5 entsprechen den Kategorien 1 - 5. 
In Tab. 2.2 werden als Beispiel die höchstzulässigen Anzeigenmerkmale bei der Ultraschallprüfung aus der [DIN 1690-2:1985] abgebildet. Bei den Anzeigenmerkmalen wird nicht nach Gussfehlerarten sondern nur nach Ausdehnung und Lage unterschieden. Diese höchstzulässigen Fehleranzeigen gelten nur für Fehler, deren Echoanzeigen über der Registriergrenze nach [Stahl-Eisen-Prüfblatt 1922:1985] liegen.

Da den Stahlgiessereien mit der Überarbeitung verschiedener Normen zur Durchführung von Qualitätsprüfungen und den entsprechenden Qualitätsgütestufen (z. B. Normen für zerstörungsfreie Werkstoffprüfungen) wirtschaftliche und technische Konsequenzen bevorstehen, wurde anfangs der neunziger Jahre die internationale Kommission 7.2 „Kosteneinfluss - Qualitätsgrad von Stahlgussteilen“ innerhalb des CIATF (Internationales Komitee giessereitechnischer Vereinigungen) gegründet. Das Ziel der Kommission ist es, Kriterien und Aufwendungen festzulegen, die ein bestimmtes Qualitätsniveau sicherstellen, und eine Beziehung zwischen Kosten und Qualität zu entwickeln, ohne jedoch die Kosten und Preise unter den Stahlgiessereien zu vergleichen [Buberl et al. 1995].

Für die Definition der Qualität wurden verschiedene die Qualität beeinflussende Kriterien zusammengestellt, wobei jedes Kriterium in fünf durch Grenzwerte umschriebene Qualitätsgrade eingeteilt wurde. Der Qualitätsgrad 1 bedeutet die höchste, der Grad 5 die niedrigste Stufe. Dazu werden für die einzelnen Kriterien die Aufwendungen (Kosten) ausgewertet, die zur Erreichung eines Qualitätsgrades notwendig sind. Die von der Kommission 7.2 definierten fünf Qualitätsgrade entsprechen nicht den fünf Gütestufen nach [DIN 1690-2:1985]. Die Kriterien umfassen unter anderem den Oberflächenzustand sowie Oberflächen- und Innenfehler. So wird die Beziehung zwischen Kosten und Qualität für die Erzeugung von Stahlgussteilen quantifiziert und ein relativer KostenFaktor für die einzelnen Qualitätsgrade aufgestellt. Diese Studie ergab beispielsweise, dass relativ zum Qualitätsgrad 3 die Herstellungskosten eines Gussstücks des Qualitätsgrads 1 um 24 \% höher, diejenigen für den Grad 5 um 20 \% niedriger sind. Die Beziehung zwischen Kosten und Qualität für die Erzeugung von Stahlgussteilen wird also quantitativ beschrieben, und es wird gezeigt, dass Kosten eingespart werden können, wenn die Qualität (und damit die Gütestufe) der Gussstücke entsprechend den Anforderung optimal gewählt wird.

\subsubsection{Neue europäische Normengeneration}

In den letzten Jahren wurden die Normen über die verschiedenen Prüfverfahren (Abschnitt 2.2.2) überarbeitet. Zur Oberflächenprüfung von Stahlgussstücken gilt seit 1997 für die Magnetpulverprüfung die Euronorm [SN EN 1369:1997]; für die Farbeindringprüfung gilt seit 1992 die ISO Norm [ISO 4987:1992]. Zur Überprüfung des Inneren von Stahlgussstücken gilt seit 2003 für die Ultraschallprüfung normalbeanspruchter Bauteile die Euronorm [SN EN 12680-1:2003], für diejenige hochbeanspruchter Bauteile die [SN EN 12680-2:2003]. Die Durchstrahlungsprüfung wird in der Norm [SN EN 12681:2003] behandelt.

Der wesentliche Unterschied zu den bisherigen Normen besteht darin, dass die Gütestufen direkt in der Norm des jeweiligen Prüfverfahrens umschrieben werden. Die höchstzulässigen Anzeigenmerkmale (Zulässigkeitsgrenzen) für die einzelnen Gütestufen sind sehr viel detaillierter beschrieben. Die Herstellung der Stahlgussknoten für die experimentellen Untersuchungen (siehe Abschnitt 3.2.2) beruht noch auf der in den Abschnitten 2.2.2 und 2.2.3 zitierten Normengeneration. 


\subsection{ERMÜDUNGSBEMESSUNG VON STAHLGUSSKNOTEN}

\subsubsection{Lebensdauerabschätzung}

\subsubsection{Konzept nach Seeger}

Anfang der achtziger Jahre wurde in [Bergmann et al. 1981] für die Anwendung in OffshoreKonstruktionen das Ermüdungsverhalten des Stahlgusses HOESCH Gs Ark 10 experimentell untersucht. Nach [DIN 17182:1992] trägt dieser Stahlguss heute die Bezeichnung GS-13 MnNi 64. Dieser Werkstoff wird unter anderem im Maschinen- und Anlagenbau eingesetzt. Bei diesen Untersuchungen wurde zusätzlich zu den Versuchen an Kleinproben ohne Gussfehler gezielt der Einfluss von Gussfehlern auf die Ermüdungsfestigkeit untersucht. Die Kleinproben mit Gussfehlern wurden aus Stahlgussknoten ausgearbeitet und die Fehler nach den amerikanischen Spezifikationen der ASTM [ASTM E 446-98] [ASTM E 186-98] [ASTM E 280-98] nach Fehlerart und -grösse klassifiziert. Dabei wurde zwischen Gasblasen, Einschlüssen, Lunkern und Warmrissen unterschieden. Aus dem Versuchsbericht ist ersichtlich, dass nur 4 der 32 Kleinproben mit Gussfehlern der Kategorien 1 und 2, die restlichen mit Gussfehlern der Kategorien 4 und 5 behaftet waren. Wie bereits erwähnt, werden Gussfehler nach ASTM Spezifikation in Kategorien (und nicht in Gütestufen wie in [DIN 1690-2:1985]) eingeteilt. Die Kategorie 1 entspricht der kleinsten und 5 der grössten Fehlergrösse.

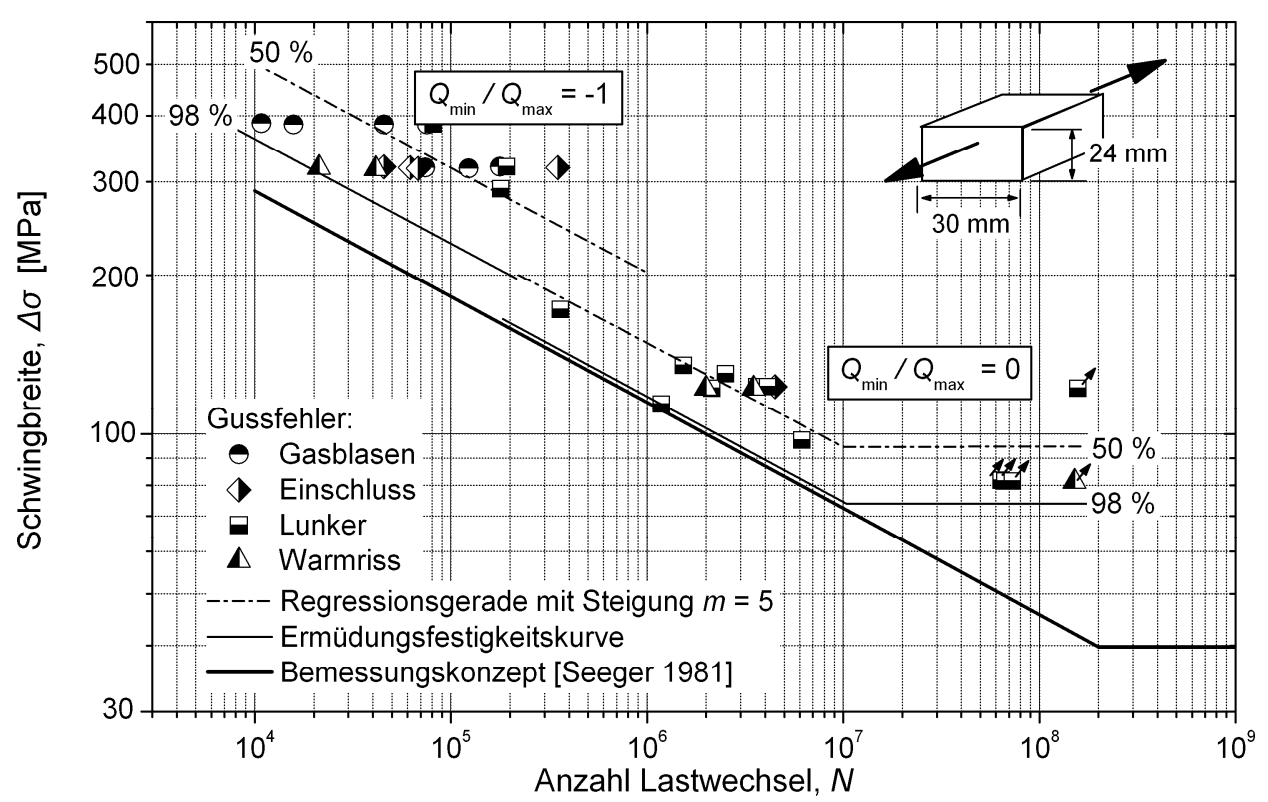

Fig. 2.9: Versuchsresultate aus [Bergmann et al. 1981]

In Fig. 2.9 werden die Versuchsresultate für die Kleinproben mit Gussfehlern aller Grössen aus [Bergmann et al. 1981] dargestellt. Die Versuche wurden für die Lastverhältnisse $Q_{\min } / Q_{\max }=-1.0$ und $Q_{\min } / Q_{\max }=0$ durchgeführt. Bei der Auswertung der Versuchsresultate wurden die Nennspannungen bezogen auf den reduzierten Querschnitt berechnet, um den Gussfehlern Rechnung zu tragen. Der Einfluss der verschiedenen Fehlerarten wurde nicht ausgewertet, da keine signifikanten Unterschiede für die Gussfehler der Kategorien 4 und 5 festgestellt werden konnten. 
Die Ermüdungsfestigkeitskurven, die für eine Überlebenswahrscheinlichkeit von $98 \%$ berechnet wurden, sind in Tab. 2.3 zusammengefasst. Die Ermüdungsfestigkeit mit zunehmendem Lastverhältnis $Q_{\min } / Q_{\max }$ ab.

\begin{tabular}{ccc}
\hline $\begin{array}{c}\Delta \sigma_{\mathrm{C}}[\mathrm{MPa}] \\
\text { bei } 2 \cdot 10^{6} \mathrm{LW}, m=5\end{array}$ & -1.0 & $\mathrm{Q}_{\min } / \mathrm{Q}_{\max }$ \\
\cline { 2 - 3 } & 172 & 0 \\
\hline Regressionsgerade $(50 \%)$ & $\mathbf{1 2 4}$ & $\mathbf{1 0 1}$ \\
\hline Ermüdungsfestigkeit $(98 \%)$ & \multicolumn{101}{c}{$\%$} \\
\hline
\end{tabular}

Tab. 2.3 : Ermüdungsfestigkeitskurve zu den Versuchsresultaten aus [Bergmann et al. 1981]

Anhand dieser Versuchsresultate wurde in [Seeger 1981] ein Bemessungskonzept für Stahlgussknoten ausgearbeitet. Dabei wird eine Ermüdungsfestigkeitskurve als Ausgangkurve festgelegt, für die bei einer Anzahl Lastwechsel $N \leq 2 \cdot 10^{8}$ gilt:

$$
\begin{gathered}
N=C \cdot \Delta \sigma^{-m} \\
\lg N=16.30-5 \cdot \lg \Delta \sigma
\end{gathered}
$$

$$
\begin{array}{ll}
m & \text { Steigung, } m=5 \\
C & \text { Konstante, } C=2 \cdot 10^{6} \cdot \Delta \sigma_{\mathrm{C}}^{m} \\
\Delta \sigma_{\mathrm{C}} & \text { Spannungsschwingbreite bei } N=2 \cdot 10^{6}, \Delta \sigma_{\mathrm{C}}=100 \mathrm{MPa}
\end{array}
$$

Bei $N>2 \cdot 10^{8}$ ist die ertragbare Spannungsschwingbreite $\Delta \sigma_{\mathrm{D}}=39.8 \mathrm{MPa}$. Diese Ausgangskurve gilt nur für:

- Lastverhältnis $\mathrm{Q}_{\min } / \mathrm{Q}_{\max }=0$

- Überlebenswahrscheinlichkeit von $98 \%$

- Wanddicke $t=25 \mathrm{~mm}$

- Gussfehlergrösse der Kategorie 4 nach [ASTM E 446-98] [ASTM E 186-98]

Um den Einfluss des Lastverhältnisses, der Wanddicke, der Fehlerkategorie und der Zugänglichkeit des Knotens für Überwachung und Reparatur zu berücksichtigen, werden Korrekturfaktoren für $\Delta \sigma_{\mathrm{C}}$ eingeführt:

$$
\Delta \tilde{\sigma}_{\mathrm{C}}=\lambda_{\mathrm{R}} \cdot \lambda_{\mathrm{F}} \cdot \lambda_{\mathrm{W}} \cdot \lambda_{\mathrm{I}} \cdot \Delta \sigma_{\mathrm{C}}
$$

$\lambda_{\mathrm{R}} \quad$ Korrekturfaktor für das Lastverhältnis;

$$
\lambda_{\mathrm{R}}=0.85 \text { wenn } \mathrm{Q}_{\min } / \mathrm{Q}_{\max }>0, \lambda_{\mathrm{R}}=1.0 \text { wenn } \mathrm{Q}_{\min } / \mathrm{Q}_{\max } \leq 0
$$

$\lambda_{\mathrm{F}} \quad$ Korrekturfaktor für die Gussfehlerkategorie; $\lambda_{\mathrm{F}}=0.85^{j-4}, j$ kennzeichnet die Gussfehlerkategorie 1 bis 5 nach [ASTM E 446-98] [ASTM E 186-98] [ASTM E 280-98]

$\lambda_{\mathrm{W}} \quad$ Korrekturfaktor für die Wanddicke; $\lambda_{\mathrm{W}}=(25 / t)^{0.15}$

$\lambda_{\mathrm{I}} \quad$ Korrekturfaktor für die Zugänglichkeit des Knotens für Überwachung und Reparatur

Der positive Einfluss des Lastverhältnisses $\mathrm{Q}_{\min } / \mathrm{Q}_{\max } \leq 0$ wird nicht berücksichtigt. Hingegen wird bei $\mathrm{Q}_{\min } / \mathrm{Q}_{\max }>0$ die Ermüdungsfestigkeit auf $85 \%$ herabgesetzt. Für Gussfehler der Kategorien $j<4$ wird die Ermüdungsfestigkeit erhöht, für Wanddicken $t>25 \mathrm{~mm}$ wird sie abgemindert. Die Einflüsse der Fehlergrösse und der Wanddicke werden auf bruchmechanischer Basis nachgewiesen. Die Fehlerart (Gasblasen, nichtmetallische Einschlüsse, Lunker, Risse) wird bei diesem Nachweis nicht berücksichtigt. Auf Grund der oft schwer zugänglichen Stahlgussknoten ist der Faktor $\lambda_{\mathrm{I}}$ insbesondere für Offshore-Konstruktionen wichtig. Im Brückenbau ist er weniger relevant, weshalb 
hier nicht näher darauf eingegangen wird. In der Richtlinie [UEG 1985] für Stahlgussknoten in Offshore-Konstruktionen wird dieses Bemessungskonzept übernommen.

Auch im Brückenbau fand dieses Bemessungskonzept bereits Verwendung [Schlaich et al. 1999.1] [Dahl et al. 1995]. Voraussetzung für die Anwendung ist, dass die Gütestufen 1 - 5 nach den technischen Lieferbedingungen [DIN 1690-2:1985] den Kategorien 1-5 nach [ASTM E 446-98] [ASTM E 186-98] [ASTM E 280-98] entsprechen (vergleiche Abschnitt 2.2.3). Nach diesem Konzept zeichnen sich die Gütestufen 1, 2 und 3, welche für die in Abschnitt 2.1.2 beschriebenen Brücken gewählt wurden, durch eine ertragbare Spannungsschwingbreite nach Gleichung (2.3) von $\Delta \sigma_{\mathrm{C}}=163 \mathrm{MPa}$ (Gütestufe 1), $\Delta \sigma_{\mathrm{C}}=138 \mathrm{MPa}$ (Gütestufe 2) und $\Delta \sigma_{\mathrm{C}}=117 \mathrm{MPa}$ (Gütestufe 3) bei einer Anzahl Schwingungen von $N=2 \cdot 10^{6}$ aus.

\subsubsection{Weitere Ermüdungsfestigkeitskurven in der Literatur}

In [Ma et al. 1997] und [OTH 92 390:1999] werden stahlgusstypische Fehler, die bei der Qualitätsüberprüfung übersehen werden können, direkt in der Ermüdungsfestigkeitskurve für Stahlgussknoten berücksichtigt, indem diese mit einem Abstand von vier Standardabweichungen von der aus Versuchsresultaten ermittelten Regressionsgerade festgelegt wird. Die mitberücksichtigten Fehlerarten und -grössen werden nicht genauer umschrieben. Die Ermüdungsfestigkeitskurve wird wie folgt festgelegt:

$$
\lg N=15.17-4 \cdot \lg \Delta \sigma
$$

Der Wanddickeneinfluss geht mit dem Faktor $(38 / t)^{0.15}$ ein, wenn die Wanddicke $t>38 \mathrm{~mm}$ ist.

In [DNV 2001] empfiehlt Det Norske Veritas für Stahlguss die Ermüdungsfestigkeitskurve C. Der Wanddickeneinfluss wird ebenfalls mit dem Faktor $(38 / t)^{0.15}$ berücksichtigt, wenn die Wanddicke $t$ $>38 \mathrm{~mm}$ ist. Der Einfluss von Gussfehlern ist nicht mitberücksichtigt.

$$
\lg N= \begin{cases}12.592-3 \cdot \lg \Delta \sigma & \text { für } N \leq 1 \cdot 10^{7} \\ 16.320-5 \cdot \lg \Delta \sigma & \text { für } N>1 \cdot 10^{7}\end{cases}
$$

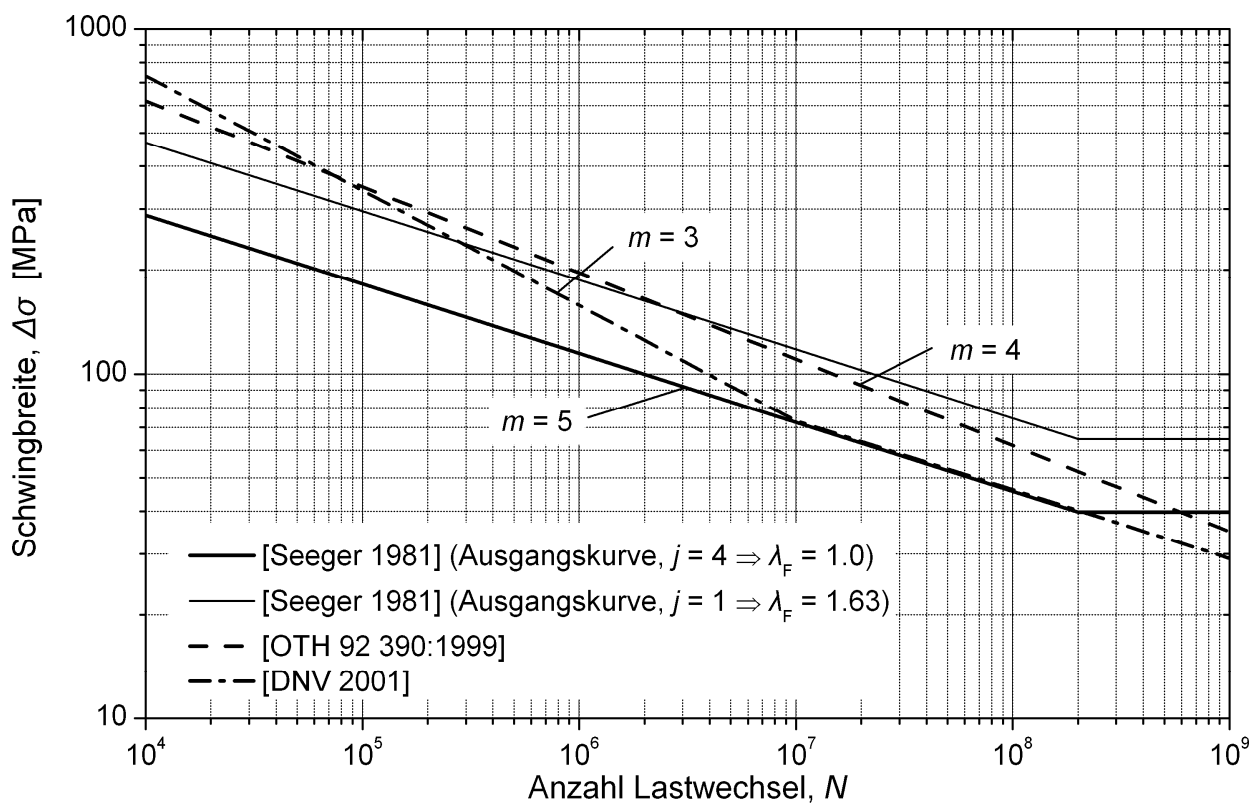

Fig. 2.10: Zusammenfassung einiger Ermüdungsfestigkeitskurven für Stahlguss 
In Fig. 2.10 und Tab. 2.4 werden die Angaben zu den Ermüdungsfestigkeitskurven zusammengefasst. Für das Bemessungskonzept [Seeger 1981] werden die Wöhlerkurven für die Fehlerkategorie $j=1$ und $j=4$ (Ausgangskurve) dargestellt. Diese beiden Wöhlerkurven umhüllen im Bereich $1 \cdot 10^{6}<N \leq 2 \cdot 10^{8}$ die Kurven nach [OTH 92 390:1999] und [DNV 2001]. Der Vergleich der Kurven zeigt, dass die Ermüdungsfestigkeit auf Grund sehr unterschiedlicher Steigungen $m$ und Schwingbreiten $\Delta \sigma_{\mathrm{C}}$ sehr unterschiedlich ausfällt.

\begin{tabular}{lccccc}
\hline Literaturreferenz & Stahlguss & $\begin{array}{c}\text { Berücksichtigung der } \\
\text { Gussfehler }\end{array}$ & $\begin{array}{c}\Delta \sigma_{\mathrm{C}} \text { bei 2·10 } \\
{[\mathrm{MPa}]}\end{array}$ & $\begin{array}{c}\text { Steigung } \\
m\end{array}$ & $\begin{array}{c}\text { Faktor für } \\
\text { Wanddickenein- } \\
\text { fluss }\end{array}$ \\
\hline [Seeger 1981] & GS-13 MnNi 64 & ja & $100^{1}$ & 5 & $(25 / t)^{0.15}$ \\
\hline [OTH 92 390:1999] & keine Angaben & ja & 165 & 4 & $(38 / t)^{0.15}$ \\
\hline [DNV 2001] & keine Angaben & nein & 125 & 3 & $(38 / t)^{0.15}$ \\
\hline
\end{tabular}

${ }^{1}$ für die Ausgangskurve $\left(j=4 \Rightarrow \lambda_{\mathrm{F}}=1.0\right)$

Tab. 2.4 : Zusammenfassung einiger Ermüdungsfestigkeitskurven für Stahlguss

\subsubsection{Rissfortschrittsparameter}

In [Blair et al. 1995] werden die Rissfortschrittsparameter für verschiedene Stahlgusssorten angegeben. Deren Rissfortschrittsverhalten entspricht mindestens demjenigen von ferritisch-perlitischen Stählen nach [Barsom 1971]. Demnach kann die konservative Annahme gemacht werden, dass allgemein für Stahlguss die für ferritisch-perlitische Stähle geltenden Rissfortschrittsparameter verwendet werden können. Nach Anpassung an die SI Einheiten gilt die Rissfortschrittsgleichung:

$$
\frac{\mathrm{d} a}{\mathrm{~d} N}=C \cdot \Delta K^{m}
$$

$$
\begin{array}{ll}
C & \text { Rissfortschrittsparameter, } C=2 \cdot 10^{-13}(\mathrm{~mm} / \text { Lastwechsel }) \cdot\left(\mathrm{N} \cdot \mathrm{mm}^{-3 / 2}\right)^{-m} \\
m & \text { Rissfortschrittsparameter, Steigung } m=3 \\
a & \text { Risstiefe }[\mathrm{mm}] \\
\Delta K & \text { Differenz des Spannungsintensitätsfaktors }\left[\mathrm{N} \cdot \mathrm{mm}^{-3 / 2}\right]
\end{array}
$$

\subsubsection{Untersuchungen zum Einfluss der Gussfehler auf die Ermüdungsfestigkeit}

Stahlgusskomponenten werden nicht nur als Konstruktionsdetails (siehe Abschnitt 2.1) sondern auch als mechanische Bauteile in den verschiedensten Anwendungsbereichen wie Automobile, Turbinen, Nuklearanlagen und Flugzeugbau eingesetzt. Da die Komponenten in diesen Anwendungsbereichen hoher Ermüdungsbeanspruchung ausgesetzt sind, wurde während der letzten drei Jahrzehnte der Einfluss von Gussfehlern auf das Ermüdungsverhalten, insbesondere auf die Dauerfestigkeit (bei $N=1 \cdot 10^{7}$ Lastwechseln), untersucht. Für diese Anwendungsbereiche sind die Gussfehler sehr klein und werden als Kurzrisse klassifiziert. Den Kurzrissen werden Risstiefen unter etwa $0.5 \mathrm{~mm}$ zugeordnet [Radaj 1995], welche mit praxisüblichen zerstörungsfreien Prüfverfahren nicht sicher erfasst werden können. Am Lehrstuhl für Stahlbau (ICOM) durchgeführte Untersuchungen zeigen, dass die Grenze zwischen Kurz- und Langriss schon bei einer Risstiefe von $0.1 \mathrm{~mm}-0.2 \mathrm{~mm}$ [Peeker 1997] liegt.

Das Kurzrissverhalten hat eine grosse Bedeutung für die Abschätzung der Lebensdauer von mechanischen Bauteilen und Proben auf der Basis der technischen Risseinleitung und für die zugehö- 
rigen Hypothesen der Schadensakkumulation. In [Murakami et al. 1994] wird eine sehr umfassende Zusammenfassung über Modelle zur Vorhersage der Ermüdungsfestigkeit für kleine Fehler, nicht-metallische Einschlüsse und Inhomogenitäten gegeben. Die Modelle werden in drei Kategorien eingeteilt: (1) empirische Modelle; (2) Modelle, die auf dem Kerbwirkungszahl-Verfahren beruhen; (3) bruchmechanische Modelle. Detaillierte Informationen zu den verschiedenen Modellen finden sich in [Radaj 1995]. Untersuchungen zum Anrissverhalten an oberflächennahen Fehlstellen im Stahlguss bei Schwingungsbeanspruchung können unter anderem in [Heuler et al. 1991] [Heuler et al. 1991.1] [Heuler et al. 1991.2] nachgelesen werden.

Im Brückenbau interessiert in erster Linie die Frage, unter welchen Bedingungen sich ein vorhandener Anriss weiter ausbreitet oder aufgefangen werden kann und ab wann ein instabiles Risswachstum eintritt. Aus diesem Grund ist hier insbesondere das Langrissverhalten relevant. Dieses wird mit der technischen Bruchmechanik erfasst. Wenn ein elastisches Werkstoffverhalten vorliegt und die plastische Zone um die Rissspitze klein gegenüber der Risslänge ist, so wird die linear elastische Bruchmechanik angewendet. Bei sehr duktilem Werkstoff werden elastisch-plastische Konzepte (elastisch-plastische Bruchmechanik) herangezogen.

\subsubsection{Berechnung der Spannungskonzentrationsfaktoren}

Für geschweisste Knoten in Offshore-Konstruktionen wurden zur Berechnung der Spannungskonzentrationsfaktoren (SCF) Parametergleichungen entwickelt, die von den Abmessungen, den Verhältnissen zwischen den Wanddicken und den Durchmessern der Hohlprofile abhängen. Da diese Parameter für die Form der Stahlgussknoten nicht exakt definiert werden können, sind die für geschweisste Knoten entwickelten Gleichungen nicht automatisch für Stahlgussknoten anwendbar.

In [Edwards et al. 1981] und [Edwards et al. 1985] wurden für Stahlgussknoten unterschiedlicher Geometrie Untersuchungen durchgeführt mit dem Ziel, Parametergleichungen für die SCF aufzustellen. Es zeigte sich aber, dass FE-Berechnungen zur Bestimmung der lokalen Spannungsspitzen unumgänglich sind. Zahlreiche FE-Untersuchungen von Stahlgussknoten bestätigen die im Vergleich zu geschweissten Knoten niedrigeren Spannungskonzentrationen. Die SCF liegen für optimierte Stahlgussknoten fast ausnahmslos unter dem Wert von 2 [Wood et al. 1982] [Nakamura et al. 1987] [Wood 1993] [Broughton et al. 1997]. Dagegen können die SCF für geschweisste Knoten Werte bis 8 annehmen [Wood et al. 1982] [Edwards et al. 1985].

\subsection{ERMÜDUNGSBEMESSUNG VON SCHWEISSNAHTANSCHLÜSSEN BEI STAHLGUSS- KNOTEN}

\subsubsection{Abschätzung der Lebensdauer mit Ermüdungsfestigkeitskurven}

Wenn die Einwirkungsgeschichte hinreichend genau bekannt ist, wird in der Regel ein Ermüdungsnachweis mit Hilfe von Ermüdungsfestigkeitskurven (Wöhlerkurven) erfolgen. Diese sind in den Normen für eine grosse Anzahl von Konstruktionsdetails angegeben. Eine Ermüdungsfestigkeitskurve beschreibt den Zusammenhang zwischen der Spannungsschwingbreite und der charakteristischen Lebensdauer. Der für eine Kurve charakteristische Wert der ertragbaren Spannungsschwingbreite für eine Lebensdauer von $2 \cdot 10^{6}$ Lastwechseln wird Kerbfall genannt und für die Klassifizierung und den Vergleich von Konstruktionsdetails herangezogen. 
Die Anwendung der Wöhlerkurven erfolgt auf der Basis von Nennspannungen (auch Nominalspannungen genannt), die mit Hilfe der Festigkeitslehre aus den Schnittkräften im Bauteil berechnet werden. Spannungskonzentrationen zum Beispiel infolge von Löchern oder Steifen werden dabei nicht einbezogen. Da die Wöhlerkurve eines Konstruktionsdetails experimentell bestimmt wird, sind die Auswirkungen von Kerben bereits im Versuch erfasst. Aus diesem Grund gelten die Ergebnisse der Wöhlerversuche nur für das untersuchte Konstruktionsdetail. Oft werden auch Abmessungsbereiche für ein Konstruktionsdetail angegeben, für die ein bestimmter Kerbfall anwendbar ist.

\subsubsection{Bestimmung des Kerbfalls}

Bei Fachwerkkonstruktionen aus Rundhohlprofilen sind die Verbindungen zwischen dem Stahlgussknoten und den Hohlprofilen im Regelfall Stumpfschweissnähte. Die Stumpfschweissnähte können entweder mit oder ohne Schweissbadsicherung ausgebildet sein. Nachfolgend werden die Kerbfälle aus verschiedenen Normen gegenübergestellt. Diese Kerbfälle gelten für Stumpfnähte ohne exzentrische Ausrichtung. Liegt eine solche vor, muss sie mit Spannungskonzentrationsfaktoren berücksichtigt werden.

\section{SIA 263 [SIA 263]}

In der Schweizer Norm für Stahlbau wird ein Kerbfall für den Querstoss von Rundhohlprofilen von gleicher Wanddicke und mit Schweissbadsicherung angegeben (Tab. 2.5). Für Wanddicken $t>25 \mathrm{~mm}$ wird der Kerbfall reduziert. Zur Schweissposition, exzentrischem Anschluss der Rundhohlprofile, Ausbildung ohne Schweissbadsicherung und Toleranzen werden keinen Angaben gemacht.

\begin{tabular}{cccc}
\hline $\begin{array}{c}\text { Kerbgruppe } \\
\Delta \sigma_{\mathrm{C}}\left[\mathrm{N} / \mathrm{mm}^{2}\right]\end{array}$ & Spezialbedingungen & Konstruktionsdetail & Beschreibung \\
\hline 71 & Abminderung: & & Kerbfall \\
$(m=3)$ & $\begin{array}{c}t>25 \mathrm{~mm} \\
k_{\mathrm{s}}=(25 / t)^{0.2}\end{array}$ & Qühte mit Wurzelunterlage \\
& & & Querstösse von runden Rohren \\
\hline
\end{tabular}

Tab. 2.5: Auszug aus der SIA 263, Tabelle 23

\section{Eurocode 3, Teil 1-9 [prEN 1993-1-9:2003]}

Im Eurocode 3 entspricht die Stumpfnaht zwischen zwei Rundhohlprofilen mit gleicher Wanddicke dem Konstruktionsdetail 3 in Tabelle 8.6 (Tab. 2.6) und wird damit als Kerbfall 71 eingestuft. Wenn die Wanddicke zwischen 8.0 und $12.5 \mathrm{~mm}$ beträgt, kann das Konstruktionsdetail zwei Stufen höher in Kerbfall 90 eingestuft werden. Folgende Stumpfnähte zwischen zwei Rundhohlprofilen sind in diesem Kerbfall nicht eingeschlossen:

- Stumpfnähte, die nicht in Wannenlage (PA) geschweisst werden. Beim Zusammenfügen einer räumlich aufgelösten Fachwerkkonstruktion mit Stahlgussknoten kann diese Bedingung nicht eingehalten werden. Sowohl im Werk als auch bei Montage auf der Baustelle müssen die Stumpfnähte auch in den Schweisspositionen PF, PG, H-L045 oder J-L045 geschweisst werden.

- Stumpfnähte, bei denen der Ermüdungsriss von der Schweissnahtwurzel ausgeht.

- Stumpfnähte mit Badsicherung.

- Stumpfnähte mit Wanddicken $t>12.5 \mathrm{~mm}$. 


\begin{tabular}{cll}
\hline Kerbfall Konstruktionsdetail & \multicolumn{1}{c}{ Beschreibung } & \multicolumn{1}{c}{ Anforderungen } \\
\hline & & $\begin{array}{l}\text { 1. Maximale Wanddicke } t \leq 12.5 \mathrm{~mm} . \\
\text { 2. Nahtüberhöhung } \leq 10 \% \text { der Schweissnahtdicke mit } \\
\text { Stösse von }\end{array}$ \\
$\begin{array}{l}\text { Rundhohlprofi- } \\
\text { len mit durchge- } \\
\text { schweissten } \\
\text { Stumpfnähten }\end{array}$ & $\begin{array}{l}\text { 3. In Wannenlage geschweisste Nähte und nachweisbar } \\
\text { frei von erkennbaren Fehlern ausserhalb der Toleranzen } \\
\text { nach EN 1090. }\end{array}$ \\
& $\begin{array}{l}\text { 4. Konstruktionsdetails mit } t>8 \text { mm dürfen 2 Kerbfall- } \\
\text { kategorien höher eingestuft werden. }\end{array}$ \\
\hline
\end{tabular}

Tab. 2.6: Auszug aus dem Eurocode 3, Teil 1-9, Tabelle 8.6

Diese Angaben sind jedoch mit Bedacht zu benutzen. Nach der Dokumentation [CIDECT 1991] beruht dieser Kerbfall auf sehr wenigen Versuchsresultaten. Aus den Hintergrundinformationen geht hervor, dass eine Auswertung der Versuchsresultate mit einer Steigung der Wöhlerkurve $m=3$ einen Kerbfall 46, mit einer Steigung $m=5$ einen Kerbfall 84 ergibt. Die Einstufung des Konstruktionsdetails in den Kerbfall 90 für Wanddicken $t>8 \mathrm{~mm}$ beruht nach [CIDECT 1991] auf Versuchsresultaten an Querstössen zwischen Rechteckhohlprofilen und nicht zwischen Rundhohlprofilen.

Zur Berechnung der SCF in der Stumpfnaht, die sich aus der exzentrische Ausrichtung zwischen den Hohlprofilen ergeben, werden in [prEN 1993-1-9:2003] keine Angaben gemacht, ausser dass die Toleranzen im Kerbfall inbegriffen sind. Hinweise für exzentrische Anschlüsse werden für ebene Blechstösse in Tabelle 8.3 für Detail 17 gegeben. Es handelt sich beim angegebenen Korrekturfaktor $k_{\mathrm{s}}$ fälschlicherweise um den SCF. Da der Korrekturfaktor $k_{\mathrm{s}}$ den Kerbfall betrifft, muss der Kehrwert $k_{s}^{-1}$ verwendet werden:

$$
k_{s}=\left(1+\frac{6 e}{t_{1}} \frac{t_{1}^{1.5}}{t_{1}^{1.5}+t_{2}^{1.5}}\right)\left(\frac{25}{t_{1}}\right)^{0.2}
$$

$e \quad$ Exzentrizität zwischen den Wanddickenmitten der Bleche

$t_{1}, t_{2} \quad$ Wanddicken der Bleche wobei $t_{2} \geq t_{1}$

Kann bei einseitig geschweissten Blechstössen ohne Schweissbadsicherung ein vollständiger Einbrand und die Verschmelzung des Schweiss- und Grundwerkstoffs nicht garantiert werden, so muss die Schweissnaht in den Kerbfall 36 (Blechstoss mit Wurzelriss) eingestuft werden. Wenn jedoch mit zerstörungsfreier Prüfung eine durchgeschweisste Naht sichergestellt wird, kann das Detail in den Kerbfall 71 (Blechstoss mit Riss am Schweissnahtfuss) werden. Die Korrektur für die Bauteildicke $\left(25 / t_{1}\right)^{0.2}$ muss berücksichtigt werden.

Kann demnach bei Stumpfnähten zwischen Rundhohlprofilen ohne Schweissbadsicherung eine voll durchgeschweisste Naht nicht garantiert werden, so müsste das Konstruktionsdetail ebenfalls in den Kerbfall 36 abgestuft werden.

\section{Det Norske Veritas, DNV-RP-C203 [DNV 2001]}

In den Empfehlungen [DNV 2001] für Offshore-Bauwerke aus Stahl werden in Tabelle 9 (Tab. 2.7) Angaben zu Stumpfnähten mit und ohne Badsicherung gemacht. Für die Anwendung dieser Tabelle im Brückenbau sind nur die einseitig geschweissten Stumpfnähte relevant (Nummer 6 und 7 in der Spalte „Description“), da eine Gegenschweissung auf der Innenseite auf Grund der kleinen 
Durchmesser nicht möglich ist. Die Schweissposition ist in der Tabelle nicht definiert. Es ist zu erkennen, dass die Badsicherung eine Verbesserung des Kerbfalls bewirkt (vergleiche Tab. 2.9).

\begin{tabular}{|c|c|c|c|}
\hline $\begin{array}{c}\text { Detail category }^{2} \\
\quad m=3\end{array}$ & Construction details & Description & Requirements \\
\hline $\mathrm{C} 1$ & \multirow{4}{*}{$P$} & $\begin{array}{l}\text { 3. Circumferential butt } \\
\text { weld made from both sides } \\
\text { dressed flush }\end{array}$ & \multirow{4}{*}{$\begin{array}{l}\text { 3. - 6.: } \\
\text { The applied stress must include the } \\
\text { SCF to allow for any thickness } \\
\text { change and for fabrication toler- } \\
\text { ances. } \\
\text { The requirements to the corre- } \\
\text { sponding detail category in Table } 5 \\
\text { apply (only for 3.-5.). }\end{array}$} \\
\hline $\mathrm{D}$ & & $\begin{array}{l}\text { 4. Circumferential butt } \\
\text { weld made from both sides }\end{array}$ & \\
\hline E & & $\begin{array}{l}\text { 5. Circumferential butt } \\
\text { weld made from both sides } \\
\text { made at site }\end{array}$ & \\
\hline $\mathrm{F}$ & & $\begin{array}{l}\text { 6. Circumferential butt } \\
\text { weld made from one side } \\
\text { on a backing bar }\end{array}$ & \\
\hline \multirow[t]{2}{*}{ F3 } & $P$ & \multirow[t]{2}{*}{$\begin{array}{l}\text { 7. Circumferential butt weld } \\
\text { made from one side without } \\
\text { a backing bar }\end{array}$} & \multirow{2}{*}{$\begin{array}{l}\text { 7.: The applied stress must include } \\
\text { the SCF to allow for any thickness } \\
\text { change and for fabrication toler- } \\
\text { ances. } \\
\text { The weld root proved free from de- } \\
\text { fects larger than } 1-2 \mathrm{~mm} \text {. }\end{array}$} \\
\hline & & & \\
\hline
\end{tabular}

Tab. 2.7: Auszug aus DNV-RP-C203, Tabelle 9

Zur Berechnung der SCF in der Stumpfnaht gibt [DNV 2001] eine Gleichung an (Abschnitt 2.4.3), die die exzentrische Ausrichtung zwischen den Hohlprofilen berücksichtigt. In den Kerbfallkategorien der Tab. 2.7 ist bereits eine exzentrische Ausrichtung von $\delta_{0}=0.1 t$ inbegriffen.

\begin{tabular}{|c|c|c|c|c|c|c|c|}
\hline $\begin{array}{l}\text { Product } \\
\text { form }\end{array}$ & $\begin{array}{l}\text { Location of po- } \\
\text { tential crack } \\
\text { initiation }\end{array}$ & $\begin{array}{l}\text { Dimensional } \\
\text { requirements }\end{array}$ & $\begin{array}{c}\text { Manufacturing } \\
\text { requirements }\end{array}$ & $\begin{array}{c}\text { Special } \\
\text { inspections re- } \\
\text { quirements }\end{array}$ & $\begin{array}{c}\text { Type } \\
\text { number }\end{array}$ & $\begin{array}{l}\text { Class }^{1} \\
m=3\end{array}$ & Sketch \\
\hline \multirow{3}{*}{$\begin{array}{l}\text { Steel } \\
\text { tubes }\end{array}$} & \multirow{3}{*}{$\begin{array}{l}\text { At the toes of } \\
\text { circumferential } \\
\text { butt welds in } \\
\text { tubes }\end{array}$} & - & $\begin{array}{l}\text { Weld made from } \\
\text { both sides }\end{array}$ & - & 7.3 & E & \\
\hline & & - & $\begin{array}{l}\text { Weld made from } \\
\text { one side on perma- } \\
\text { nent backing strip }\end{array}$ & - & 7.4 & $\mathrm{~F}$ & \\
\hline & & - & $\begin{array}{l}\text { Weld made from } \\
\text { one side with no } \\
\text { backing strip }\end{array}$ & $\begin{array}{l}\text { Weld to be } \\
\text { checked for full } \\
\text { penetration }\end{array}$ & 7.5 & F2 & \\
\hline
\end{tabular}

Tab. 2.8 : Auszug aus BS $7608: 1993$, Tabelle 7

\section{British Standard BS 7608:1993 [BS 7608:1993]}

In der englischen Norm für Stahlkonstruktionen werden ebenfalls Kerbfälle zur einseitig geschweissten Stumpfnaht zwischen Rundhohlprofilen mit und ohne Badsicherung angegeben. Allerdings wird hier nur darauf hingewiesen, dass die SCF berücksichtigt werden müssen, ohne genauere Angaben zu deren Berechnung zu machen. Tab. 2.8 zeigt einen Ausschnitt der Tabelle 7 aus [BS 7608:1993] mit den Kerbfällen für verschiedene Stumpfnahtausbildungen. Wie in [DNV 2001] bewirkt die Badsicherung eine Verbesserung des Kerbfalls (vergleiche Tab. 2.9).

\footnotetext{
${ }^{2}$ Für die Zuordnung zu den Kerbfällen nach Eurocode 3 siehe Tab. 2.9.
} 


\section{Zusammenfassung}

Tab. 2.9 fasst die Kerbfälle der vier oben erwähnten Normen und Empfehlungen zusammen. Für die Kerbfälle in [DNV 2001] und [BS 7608:1993] werden die äquivalenten Kerbfälle gemäss der europäischen Konvention für Stahlbau und Eurocode 3 angegeben. Die Werte aus den Quellen [SIA 263] [DNV 2001] und [BS 7608:1993] sind sehr ähnlich. Hingegen wird die Schweissnaht ohne Badsicherung in [prEN 1993-1-9:2003] in eine wesentlich höhere Kerbfallkategorie eingestuft. Diese Einstufung gilt jedoch nur für den Bereich $t \leq 12.5 \mathrm{~mm}$ und ist für den Brückenbau mit weit höheren Wanddicken nicht anwendbar.

Zur Norm [prEN 1993-1-9:2003] stehen in [CIDECT 1991] Hintergrundinformationen zum besseren Verständnis und zur Interpretation der Angaben zum Kerbfall zur Verfügung.

\begin{tabular}{ccccccccc}
\hline Bezeichnung & \multicolumn{2}{c}{ SIA 263 } & \multicolumn{2}{c}{ Eurocode 3, Teil 9 } & \multicolumn{2}{c}{ DNV-RP-C203 } & \multicolumn{2}{c}{ BS 7608:1993 } \\
\hline Badsicherung & mit & ohne & mit & ohne & mit & ohne & mit & ohne \\
\hline nach Norm & 71 & - & - & $71 / 90^{1}$ & F & F3 & F & F2 \\
\hline nach EC 3 & 71 & - & - & $71 / 90^{1}$ & 71 & 56 & 68 & 60 \\
\hline
\end{tabular}

${ }^{1}$ Konstruktionsdetails mit $8 \mathrm{~mm}<t \leq 12.5 \mathrm{~mm}$

Tab. 2.9 : Kerbfalleinordnung nach Eurocode 3

\subsubsection{Berechnung der Spannungskonzentrationsfaktoren}

Da bei einseitig geschweissten Stumpfnähten die Aussenseite qualitativ besser ist und daher die Wöhlerkurve höher ausfällt als für die Wurzelseite, empfiehlt [DNV 2001] für Offshore-Bauwerke, bei Schweissnähten mit verschiedenen Wanddicken den Wanddickenübergang (Steigung 1:4) auf die Aussenseite des Rohres zu verlegen. Auf diese Weise ist der Spannungskonzentrationsfaktor (SCF) auf der Aussenseite > 1.0, auf der Innenseite < 1.0. Oft wird für den SCF auf der Innenseite der Wert 1.0 angesetzt. Im Brückenbau ist das Verlegen des Wanddickenübergangs auf die Aussenseite aus architektonischer Sicht nicht denkbar. Daher wird hier eine schlechtere Wöhlerkurve für die Innenseite mit einem SCF $>1.0$ kombiniert.

Wie erwähnt, gibt [DNV 2001] zur Berechnung des SCF in der Stumpfnaht eine Gleichung an, die die exzentrische Ausrichtung zwischen den Hohlprofilen berücksichtigt. Die Gleichung wird in [Lotsberg 1998] hergeleitet und gilt für SCF bei Stumpfnähten mit Wanddickenübergang auf der Innenseite:

$$
\mathrm{SCF}=1+\frac{6\left(\delta_{\mathrm{t}}+\delta_{\mathrm{m}}-\delta_{0}\right)}{t} \cdot \frac{1}{1+\left(\frac{T}{t}\right)^{2.5}} \cdot e^{-\alpha}
$$

mit

$$
\alpha=\frac{1.82 \cdot L}{\sqrt{D \cdot t}} \cdot \frac{1}{1+\left(\frac{T}{t}\right)^{2.5}}
$$

$t \quad$ kleinere Wanddicke der beiden Hohlprofile [mm]

$T$ grössere Wanddicke der beiden Hohlprofile [mm]

D Aussendurchmesser der Hohlprofile [mm] 
$L \quad$ Länge, auf welcher die Exzentrizität verläuft [mm]

$\delta_{\mathrm{t}}, \delta_{\mathrm{m}}, \delta_{0} \quad$ Exzentrizitäten, siehe Fig. $2.11[\mathrm{~mm}]$

In den Kerbfallkategorien der Tab. 2.7 ist bereits eine exzentrische Ausrichtung von $\delta_{0}=0.1 t$ inbegriffen. Wenn die exakte Exzentrizität berechnet wird, so wird $\delta_{0}$ von der Summe der exzentrischen Ausrichtung subtrahiert. Eine exzentrische Ausrichtung kann folgende Ursachen haben:

- verschiedene Radien bei gleicher Wanddicke der Hohlprofile, Fig. 2.11a)

- verschiedene Wanddicken bei gleichem Aussenradius der Hohlprofile, Fig. 2.11b)

- ovale Kreisquerschnitte, Fig. 2.11c)

- Verschiebung eines Kreisquerschnitts in Bezug auf die Achse des anderen Querschnitts, Fig. 2.11d)

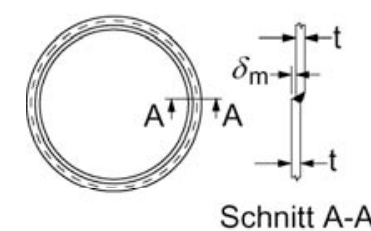

a)

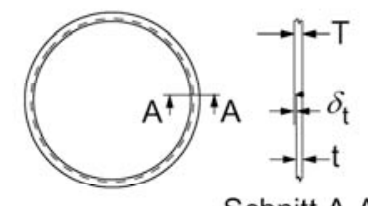

b)

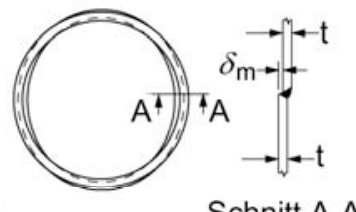

c)

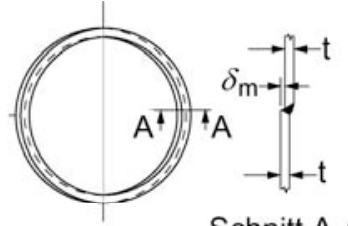

Schnitt A-A

d)

Fig. 2.11: Ursachen für exzentrische Anschlüsse (Auszug [DNV 2001])

\subsection{ZUSAMMENFASSUNG UND FOLGERUNGEN}

In diesem Kapitel wird zunächst die Entwicklung der Stahlgussknoten als Konstruktionsdetail in Offshore-Bauwerken und im Stahlhoch- und Brückenbau beschrieben. Nach einer allgemein gehaltenen Einführung in für Gussstücke typische Gussfehler werden die Prüfmethoden zum Nachweis dieser Fehler aufgeführt und die aktuelle Liefernorm [DIN 1690-2:1985] für Stahlgussbauteile erklärt.

Eine Mitte der Neunzigerjahre durchgeführte Studie zeigt die Beziehung zwischen Kosten und Qualität von Stahlgussteilen auf. Es kann Folgendes daraus geschlossen werden:

- Dank dieser Studie wird die Beziehung zwischen Kosten und Qualität für die Erzeugung von Stahlgussteilen quantitativ beschreiben, und es wird gezeigt, dass Kosten eingespart werden können, wenn die Qualität (und damit indirekt die Gütestufe) der Gussstücke entsprechend den Anforderung optimal gewählt wird.

Nach einem kurzen historischen Überblick werden die derzeit im Brückenbau angewandte Ermüdungsbemessung und der Zusammenhang zu den Qualitätsanforderungen - und somit zu den zulässigen Gussfehlern - nach der Liefernorm aufgezeigt. Es kann Folgendes daraus geschlossen werden:

- Derzeit werden generell für Stahlgussknoten im Brückenbau eine Mindestgütestufe 3 für den Knotenbereich und 1 bzw. 2 für die Anschweissenden gewählt. Nach dem Bemessungskonzept in [Seeger 1981] ergeben diese Gütestufen eine sehr hohe Ermüdungsfestigkeit von $\Delta \sigma_{\mathrm{C}}=163 \mathrm{MPa}$ (Gütestufe 1), $\Delta \sigma_{\mathrm{C}}=138 \mathrm{MPa}$ (Gütestufe 2) und $\Delta \sigma_{\mathrm{C}}=117 \mathrm{MPa}$ (Gütestufe 3) bei einer Anzahl Schwingungen von $N=2 \cdot 10^{6}$. 
Das Ermüdungsverhalten von Stahlgussknoten wird wesentlich von den Schweissnahtanschlüssen zwischen den Stahlgussknoten und den Hohlprofilen beeinflusst. Eine richtige Einschätzung der Ermüdungsfestigkeit der Stumpfnähte ist daher sehr wichtig. Aus diesem Grund wird hier eine Zusammenfassung aus einigen Normen zu Stumpfnähten gemacht. Es kann Folgendes daraus geschlossen werden:

- Die Angaben zur Ermüdungsfestigkeit von Stumpfnähten zwischen Hohlprofilen ohne Schweissbadsicherung sind nicht kohärent. Es wird je nach Norm Kerbfall 56 bis Kerbfall 90 angegeben. [SIA 263] enthält keine Angaben zu diesem Konstruktionsdetail.

- Die Angaben zur Ermüdungsfestigkeit von Stumpfnähten zwischen Hohlprofilen mit Schweissbadsicherung zeigen eine leicht höhere Ermüdungsfestigkeit im Vergleich zu Nähten ohne Schweissbadsicherung. In [prEN 1993-1-9:2003] werden für dieses Konstruktionsdetail keine Angaben gemacht.

\section{Fazit:}

Eine wirtschaftlich optimale Bemessung eines Stahlgussknotens ist dann gegeben, wenn der Ermüdungswiderstand des Gussknotens demjenigen der Anschlussnähte entspricht. In Anbetracht der niedrigen Ermüdungsfestigkeiten der Stumpfnähte ist zu vermuten, dass die derzeit gängigen Gütestufen für die realen Anforderungen bei der Anwendung in Brücken zu hoch angesetzt sind. 


\section{EXPERIMENTELLE UNTERSUCHUNGEN}

\subsection{EINLEITUNG}

Um das Ermüdungsverhalten von Stahlgussknoten als Komponenten eines Fachwerkträgers aus Stahlhohlprofilen zu untersuchen, werden grossmassstäbliche Versuche durchgeführt. Ziel der Untersuchungen ist es, für verschiedene Details in den Probekörpern die Anzahl Lastwechsel bis zum Bruch zu bestimmen. Dabei werden die Dehnungen in den Hohlprofilen, am Schweissnahtfuss der Verbindungen zwischen den Knotenstutzen und den Hohlprofilen sowie in den Gussknoten mittels Dehnmessstreifen gemessen, um den Spannungsverlauf im Gussknoten und die Beanspruchung der Schweissnähte ermitteln zu können.

Die Auswertung der Rissfortschrittsdaten soll aufzeigen, welche der beiden Komponenten Stumpfschweissnähte oder Stahlgussteil - massgebend für die Ermüdungsfestigkeit ist und erlaubt darüber hinaus eine erste Abschätzung der Ermüdungsfestigkeit der Stahlgussknoten. Dabei wird untersucht, ob und inwiefern die Gütestufe des Stahlgusses (Gussfehlergrössen an der Oberfläche und im Volumen), des Steifigkeitssprungs zwischen den Knoten und den anschliessenden Hohlprofilen und die Schweissnahtausbildung einen Einfluss auf die Ermüdungsfestigkeit des Fachwerkträgers haben.

\subsection{PROBEKÖRPER}

\subsubsection{Allgemeine Angaben}

Am Lehrstuhl für Stahlbau ICOM wurden bereits vor der vorliegenden Arbeit im Rahmen einer Dissertation numerische und experimentelle Untersuchungen zum Ermüdungsverhalten von geschweissten Knoten durchgeführt [Schumacher 2003]. Damit die Ergebnisse gegenübergestellt werden können, werden die Probekörper mit den Stahlgussknoten mit ähnlichen Abmassen hergestellt wie diejenigen dieser bereits durchgeführten experimentellen Untersuchungen.

Ein Probekörper für die Voruntersuchung, zwei Probekörper für die Serie A und drei für die Serie B werden getestet. Fig. 3.1 zeigt die Anordnung und die Abmessungen der Fachwerkträger für die Serie A. Für die Diagonalen und den Untergurt der Fachwerkträger werden Rundhohlprofile, für den Obergurt ein geschweisster Blechträger eingesetzt. Die vier Knoten im Untergurt der ebenen Fachwerkträger sind als Stahlgussknoten ausgebildet. Die Verbindungen zwischen den Diagonalen und dem Obergurt werden mittels Kopfplatten und Schraubverbindungen realisiert. Die Anordnung für die Fachwerkträger der Serie B ist vergleichbar, es sind jedoch Stahlgussknoten mit einer modifizierten Geometrie (Fig. 3.2) eingebunden und die Wanddicken der Diagonalen und des Untergurts sind angepasst.

Die Versuchskörper bestehen aus den zwei in der Mitte des Untergurts liegenden Stahlgussknoten und den anschliessenden Diagonalen und Untergurthohlprofilen. Sie sind mittels Schraubverbindungen in den Versuchsrahmen, bestehend aus dem Obergurt und auf beiden Seiten je zwei äusseren Diagonalen, eingebunden. Die Fachwerkträger werden einfach gelagert und in Feldmitte mittels zwei Hydraulikkolben mit einer Einzellast belastet. Durch die Belastung in Feldmitte und eine angemessene Wahl der Hohlprofilwanddicken werden die Probekörper stärker belastet als der Versuchsrahmen. Die Stahlgussknoten und die Schweissverbindungen zwischen den Knotenstutzen 
und den anschliessenden Hohlprofilen sind die kritischen Ermüdungsdetails. Nach abgeschlossenem Versuch wird der getestete Probekörper ausgebaut und der nächste in den unveränderten Versuchsrahmen eingebaut und verschraubt.

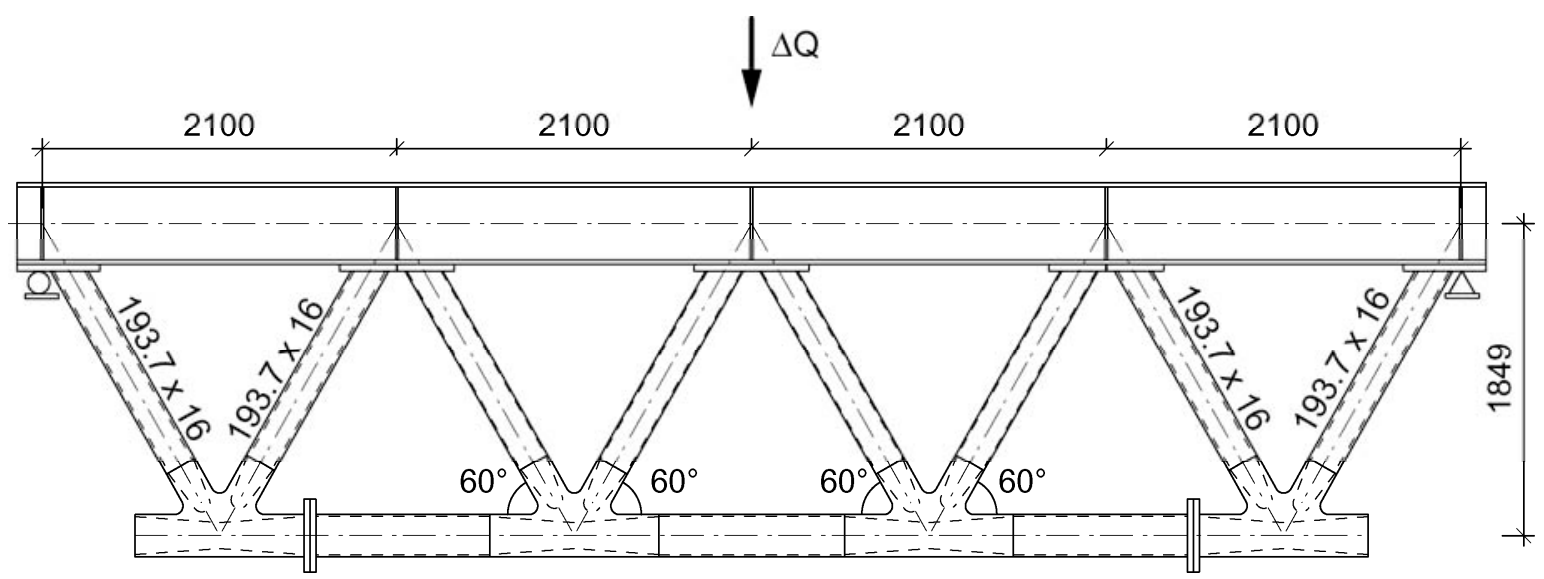

Fig. 3.1: Fachwerkträger, Anordnung und Abmessungen des Probekörpers (Mittelstück) und des Versuchsrahmens, Serie A

\subsubsection{Herstellung der Stahlgussknoten}

\subsubsection{Knotengeometrie}

Um den Zeit- und Kostenaufwand zu optimieren, wurde dem Lehrstuhl für Stahlbau (ICOM) von der Stahlgiesserei Friedrich Wilhelms-Hütte ein bereits bestehendes Holzmodell eines K-Knotens zur Verfügung gestellt. Diese Knotengeometrie wird für die Herstellung der Stahlgussknoten der Serie A verwendet. Fig. 3.2 zeigt die Längsschnitte der Stahlgussknoten beider Serien. Im Vergleich zur Geometrie der Serie A werden die Stutzen der Stahlgussknoten der Serie B auf rund 80\% verkürzt und die Wanddicken erhöht. Mit diesen Modifikationen soll der Einfluss der Geometriegestaltung der Knoten auf die durch den Steifigkeitssprung zwischen den Hohlprofilen und den Stahlgussknoten induzierten Sekundärmomente untersuchen werden.
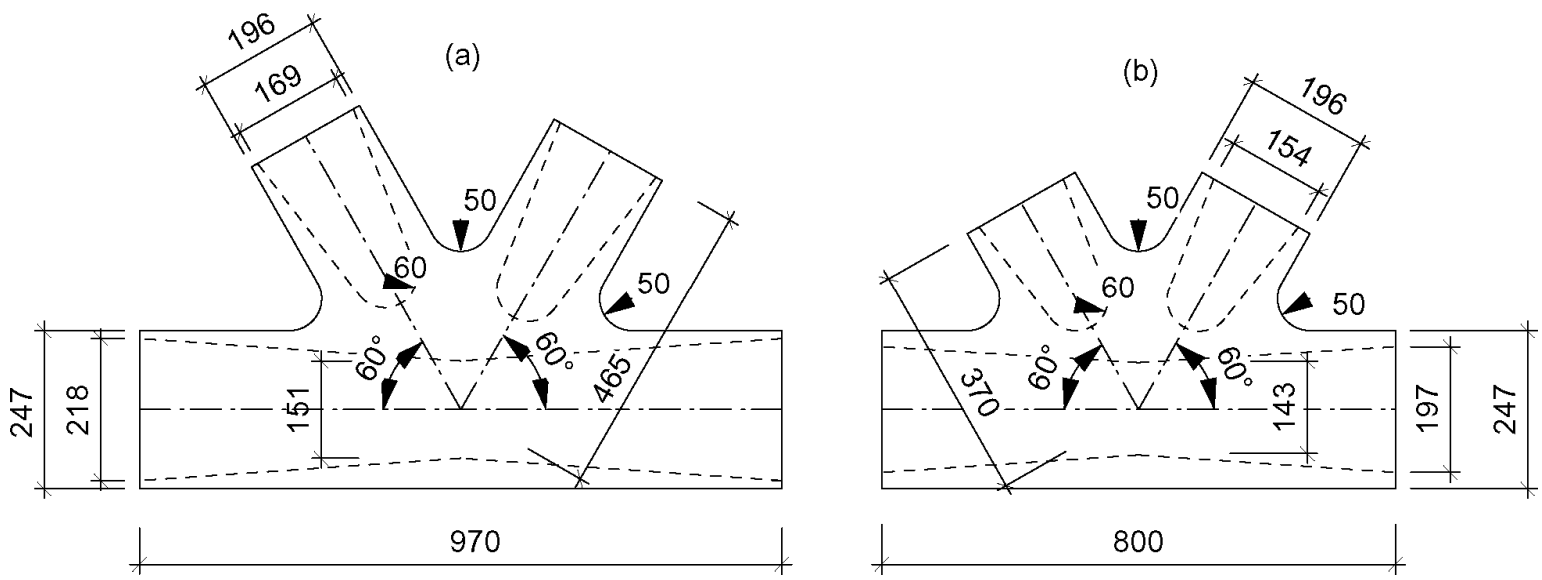

Fig. 3.2: Längsquerschnitt der Stahlgussknoten der Serie A (a) und der Serie B (b) 
Bei der Ausarbeitung der Knotengeometrie muss darauf geachtet werden, dass der Volumenschwund beim Abkühlen der Schmelze durch einen korrekt dimensionierten Speiser kompensiert wird. Um Erstarrungsrisse zu vermeiden, müssen die Wanddicken kontinuierlich in Richtung des Speisers mit einem Mindestwinkel von $4^{\circ}$ zunehmen und dürfen höchstens über eine Länge, die der dreifachen Wanddicke entspricht, konstant sein. Die Knotengeometrie wird generell immer in Zusammenarbeit mit der Giesserei festgelegt. In ihrer Verantwortung liegt es auch, den Speiser mittels einer Erstarrungssimulation korrekt zu dimensionieren.

\subsubsection{Werkstoff}

Für die Knoten wird der vergütete Stahlguss GS-20 Mn 5 V mit der Werkstoffnummer 1.1120 gemäss [DIN 17182:1992] gewählt. In Tab. 3.1 werden die genormten Grenzwerte für die chemischen Elemente wiedergegeben und den Werten aus den Werksanalysen gegenübergestellt. Die Werte wurden den mitgelieferten Abnahmeprüfzeugnissen 3.1B gemäss [EN 10204:1991/A1:1995] entnommen und erfüllen die Anforderungen.

\begin{tabular}{|c|c|c|c|c|c|c|c|c|c|}
\hline & & \multicolumn{8}{|c|}{ Massenanteil in \% } \\
\hline & & $\mathrm{C}$ & $\mathrm{Si}$ & $\mathrm{Mn}$ & $\mathrm{P}$ & $\mathrm{S}$ & $\mathrm{Cr}$ & Mo & $\mathrm{Ni}$ \\
\hline & & & $\leq$ & & $\leq$ & $\leq$ & $\leq$ & $\leq$ & $\leq$ \\
\hline \multicolumn{2}{|c|}{ DIN 17182} & 0.17 bis 0.23 & 0.60 & 1.0 bis 1.5 & 0.02 & 0.015 & 0.20 & 0.15 & 0.40 \\
\hline \multirow{2}{*}{$\begin{array}{l}\text { Werks- } \\
\text { analyse }\end{array}$} & Serie A & 0.18 & 0.36 & 1.21 & 0.01 & 0.003 & 0.09 & 0.09 & 0.35 \\
\hline & Serie B & 0.17 & 0.44 & 1.30 & 0.01 & 0.001 & 0.11 & 0.11 & 0.19 \\
\hline
\end{tabular}

Tab. 3.1: Chemische Zusammensetzung der Stahlgusssorte GS-20 Mn 5 nach [DIN 17182:1992] und nach den Abnahmeprüfzeugnissen $3.1 B$ aus dem Werk

Die mechanischen Eigenschaften des Stahlgusses GS-20 Mn 5 V gemäss [DIN 17182:1992] werden in Tab. 3.2 den Werten aus den bereits erwähnten Abnahmeprüfzeugnissen gegenübergestellt. Die Werte erfüllen ebenfalls die Anforderungen.

\begin{tabular}{cccccc}
\hline & $\begin{array}{c}\text { Wanddicke } \\
{[\mathrm{mm}]}\end{array}$ & $\begin{array}{c}0.2 \%-\text { Fliessgrenze } f_{\mathrm{y}} \\
{\left[\mathrm{N} / \mathrm{mm}^{2}\right]}\end{array}$ & $\begin{array}{c}\text { Zugfestigkeit } f_{\mathrm{u}} \\
{\left[\mathrm{N} / \mathrm{mm}^{2}\right]}\end{array}$ & $\begin{array}{c}\text { Bruchdehnung } \mathcal{E}_{\mathrm{u}} \\
{[\%]}\end{array}$ \\
\hline \multirow{2}{*}{ DIN 17 182 } & bis 50 & $\geq 360$ & $\geq 500$ bis 650 & $\geq 24$ \\
\hline \multirow{2}{*}{ Werkanalyse } & Serie A & & 476 & 592 & 24.2 \\
\cline { 2 - 6 } & Serie B & & 459 & 611 & 24.8 \\
\hline
\end{tabular}

Tab. 3.2: Mechanische Eigenschaften der Stahlgusssorte GS-20 Mn 5 nach [DIN 17182:1992] und nach den Abnahmeprüfzeugnissen $3.1 B$ aus dem Werk

In Tab. 3.3 werden die Ergebnisse der Kerbschlagarbeit für die Prüftemperaturen von $-30^{\circ} \mathrm{C}$ und Raumtemperatur für beide Chargen der Serie A und B den Normwerten gegenübergestellt. Die Werte für die Kerbschlagarbeit liegen mit $110 \mathrm{~J}$ und $122 \mathrm{~J}$ deutlich über den geforderten $27 \mathrm{~J}$. 


\begin{tabular}{|c|c|c|c|c|}
\hline & & $\begin{array}{l}\text { Wanddicke } \\
{[\mathrm{mm}]}\end{array}$ & $\begin{array}{l}\text { Prüftemperatur } T_{\text {Test }} \\
{\left[{ }^{\circ} \mathrm{C}\right]}\end{array}$ & $\begin{array}{c}\text { Kerbschlagarbeit } A_{\mathrm{v}} \\
{[\mathrm{J}]}\end{array}$ \\
\hline \multicolumn{2}{|l|}{ DIN 17182} & bis 50 & -30 & 27 \\
\hline \multirow{4}{*}{ Werkanalyse } & \multirow{2}{*}{ Serie A } & & 20 & 158 \\
\hline & & & -30 & 110 \\
\hline & \multirow{2}{*}{ Serie B } & & 20 & 172 \\
\hline & & & -30 & 122 \\
\hline
\end{tabular}

Tab. 3.3: Kerbschlagarbeit der vergüteten Stahlgusssorte GS-20 Mn 5 V nach [DIN 17182:1992] und nach den Abnahmeprüfzeugnissen $3.1 B$ aus dem Werk

Im Rahmen eines gemeinsames Forschungsprojekts ${ }^{3}$ mit der Versuchanstalt für Stahl, Holz und Steine der Universität Karlsruhe, wurde in einem externen Auftrag eine Masterkurve nach [ASTM E 1921-03 2003] für den Stahlguss GS-20 Mn 5 V erstellt. Die Masterkurve basiert auf $K_{\mathrm{Ic}}$-Werten, die wie folgt aus den $J$-Integral-Werten $J_{\text {Ic }}$ berechnet werden:

$$
K_{\mathrm{Ic}}=\sqrt{J_{\mathrm{Ic}} \frac{E}{1-v^{2}}}
$$

Ic kritischer Wert des J-Integrals bei dem es zur Rissausbreitung kommt; experimentell mit Hilfe der Risswiderstandskurve bestimmt

E Elastizitätsmodul, $E=210000 \mathrm{MPa}$

$v \quad$ Querdehnungszahl, $v=0.3$

Daraus ergibt sich bei $K_{\mathrm{Ic}}=100 \mathrm{MPa} \cdot \mathrm{m}^{0.5}=3160 \mathrm{~N} \cdot \mathrm{mm}^{-3 / 2}$ eine Bezugstemperatur $T_{0}=-155^{\circ} \mathrm{C}$. Die in Fig. 3.3 dargestellte Masterkurve aus dem Prüfbericht [IWT 2004] gilt für $25 \mathrm{~mm}$ dicke Proben. Bei ihrer Anwendung auf andere Materialdicken muss die Dickenkorrektur $(w / 25)^{1 / 4}$ angewendet werden.

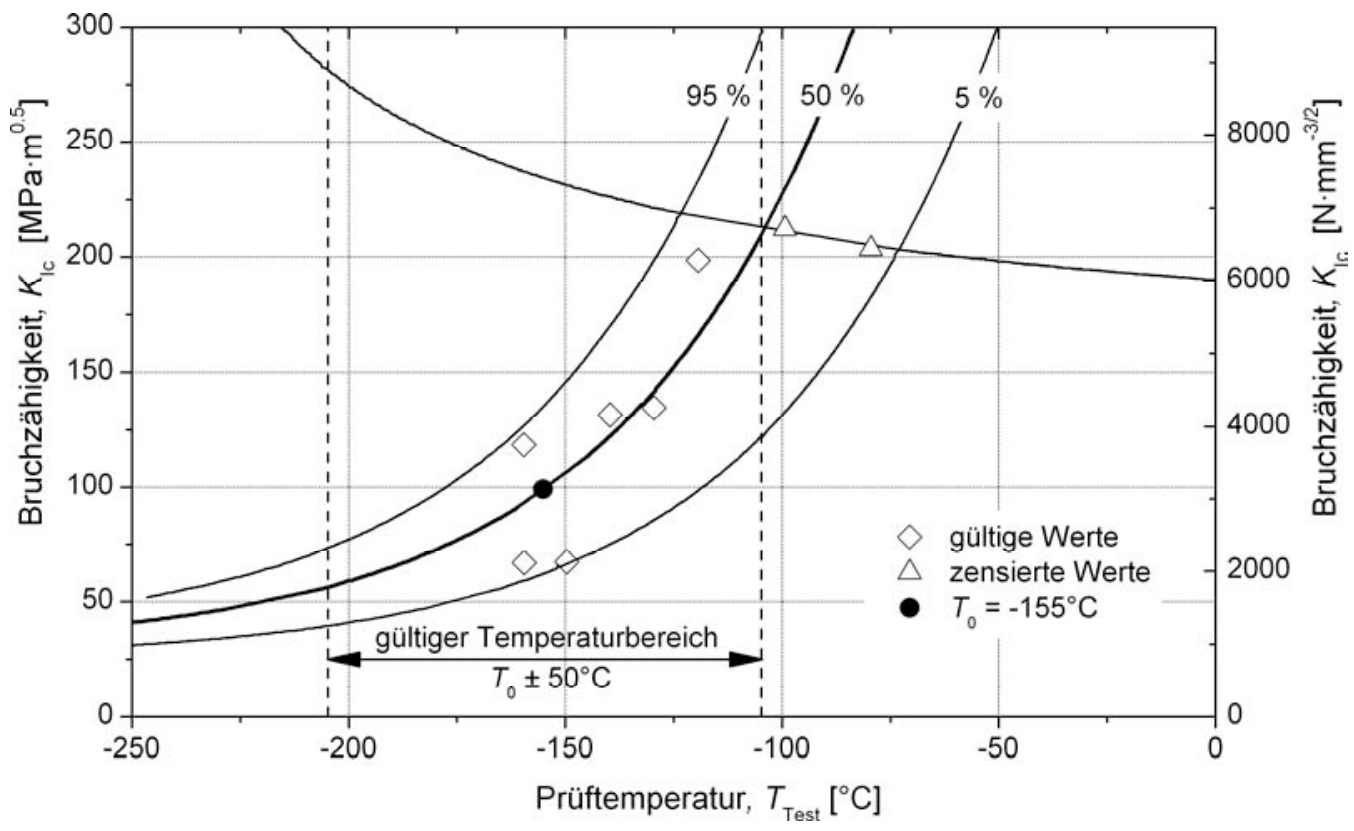

Fig. 3.3: Masterkurve für GS-20 Mn 5 V nach [IWT 2004]

\footnotetext{
${ }^{3}$ Forschungsvorhaben der FOSTA-Forschungsvereinigung Stahlanwendung e.V. P591 „Wirtschaftliches Bauen von Strassen- und Eisenbahnbrücken mit Stahlhohlprofilen“
} 
Für die Werte der Kerbschlagarbeit $A_{\mathrm{V}}$ in Tab. 3.3 kann mittels empirischen Formeln auf die Bruchzähigkeit $K_{\text {Ic }}$ geschlossen werden. Nach [Barsom et al. 1970] gilt für den Tieflage- und Temperaturübergangsbereich bei niedriger (quasi-statischer) Beanspruchungsgeschwindigkeit (Dehnrate $\dot{\varepsilon} \approx 10^{-1} / \mathrm{sec}$ ):

$$
\frac{K_{\mathrm{Ic}, \text { Test }}^{2}}{E}=0.222 \cdot A_{\mathrm{V}}^{3 / 2}
$$

$A_{\mathrm{V}} \quad$ Kerbschlagarbeit [J]

E Elastizitätsmodul, $E=210000 \mathrm{MPa}$

$K_{\text {Ic,Test }} \quad$ Bruchzähigkeit $\left[\mathrm{N} \cdot \mathrm{mm}^{-3 / 2}\right]$ bei Prüftemperatur $T_{\text {Test }}$

In [Sailors et al. 1972] wird ebenfalls eine empirische Formel zur Umrechnung angegeben:

$$
K_{\mathrm{Ic}, \mathrm{Test}}=466 \cdot \sqrt{A_{\mathrm{V}}}
$$

\begin{tabular}{|c|c|c|c|c|c|c|}
\hline & & \multirow{2}{*}{$\begin{array}{c}\text { Wanddicke } \\
\text { [mm] }\end{array}$} & \multirow{2}{*}{$\begin{array}{c}\text { Prüftemperatur } T_{\text {Test }} \\
{\left[{ }^{\circ} \mathrm{C}\right]}\end{array}$} & \multirow{2}{*}{$\begin{array}{c}\text { Kerbschlagarbeit } A_{\mathrm{V}} \\
{[\mathrm{J}]}\end{array}$} & \multicolumn{2}{|c|}{ Bruchzähigkeit $K_{\mathrm{Ic}}$} \\
\hline & & & & & $\begin{array}{c}\text { [Barsom et al. 1970] } \\
{\left[\mathrm{N} \cdot \mathrm{mm}^{-3 / 2}\right]}\end{array}$ & $\begin{array}{c}\text { [Sailors et al. 1972] } \\
{\left[\mathrm{N} \cdot \mathrm{mm}^{-3 / 2}\right]}\end{array}$ \\
\hline \multicolumn{2}{|c|}{ DIN 17182} & bis 50 & -30 & 27 & 2560 & 2400 \\
\hline \multirow{4}{*}{$\begin{array}{l}\text { Werks- } \\
\text { analyse }\end{array}$} & \multirow{2}{*}{ Serie A } & - & 20 & 158 & 9620 & 5810 \\
\hline & & - & -30 & 110 & 7330 & 4850 \\
\hline & \multirow{2}{*}{ Serie B } & - & 20 & 172 & 10250 & 6060 \\
\hline & & - & -30 & 122 & 7920 & 5110 \\
\hline
\end{tabular}

Tab. 3.4: Umrechung von der Kerbschlagarbeit $A_{\mathrm{V}}$ in Bruchzähigkeit $K_{\mathrm{Ic}}$

In [Sailors et al. 1972] wird bereits gezeigt, dass im Vergleich zur Gleichung (3.3) mit Gleichung (3.2) die $K_{\mathrm{Ic}}$ Werte bei niedriger Kerbschlagarbeit $A_{\mathrm{V}}$ unter- und bei hoher Kerbschlagarbeit überschätzt werden. Im Bereich von $A_{\mathrm{V}}=27 \mathrm{~J}$ liefern beide Gleichungen ähnliche Bruchzähigkeiten.

Demnach entspricht der Sollwert der Bruchzähigkeit bei einer Prüftemperatur von $T_{\text {Test }}=-30^{\circ} \mathrm{C}$ auf der sicheren Seite liegend $K_{\mathrm{Ic}}=2400 \mathrm{~N} \cdot \mathrm{mm}^{-3 / 2}$. Es ist aber offensichtlich, dass dies im Vergleich $\mathrm{zu}$ den Werten aus der Werksanalyse und der Masterkurve mit einer Bezugstemperatur $T_{0}=-155^{\circ} \mathrm{C}$ für $K_{\mathrm{Ic}}=3160 \mathrm{~N} \cdot \mathrm{mm}^{-3 / 2}$ ein sehr tiefer Wert ist.

Die Schweisseignung ist ein wichtiges Kriterium für die Wahl des Werkstoffs. Die Wirkung der Legierungselemente auf die (Kalt-)Rissneigung der aufgehärteten Zonen in einer Schweissnaht lässt sich zahlenmässig z. B. mit dem Kohlenstoffäquivalent angeben. Im Anhang C.2 der Norm [EN 1011-2:2001] wird für unlegierte Stähle, Feinkornbaustähle und niedriglegierte Stähle die Methode zur Vermeidung von Wasserstoffrissen beschrieben und Formel (3.4) für die Berechnung des Kohlenstoffäquivalents angegeben. Diese Grösse wird zur Bestimmung der Vorwärmetemperatur benutzt (Abschnitt 3.2.3):

$$
\mathrm{CE}=\mathrm{C}+\frac{\mathrm{Mn}}{6}+\frac{\mathrm{Cr}+\mathrm{Mo}+\mathrm{V}}{5}+\frac{\mathrm{Ni}+\mathrm{Cu}}{15}[\%]
$$

In Tab. 3.5 sind die Werte des Kohlenstoffäquivalents für die Chargen der Serie A und B zusammengefasst. Da die Werte nur knapp über dem Grenzwert von 0.4 liegen, ab dem ein Vorwärmen 
erforderlich ist, und Elektroden mit sehr geringem Wasserstoffgehalt gewählt werden, sind die Knotenstutzen ohne Vorwärmung schweissbar.

\begin{tabular}{lc}
\hline & CE [\%] \\
\hline Charge Serie A & 0.441 \\
\hline Charge Serie B & 0.443 \\
\hline
\end{tabular}

Tab. 3.5: Kohlenstoffäquivalent nach [EN 1011-2:2001]

\subsubsection{Erschmelzung}

Der Stahl wurde in einem Elektroofen erschmolzen. Während dem Erschmelzungsprozess wurde kontinuierlich die chemische Zusammensetzung des Stahlgusses gemäss der Norm [DIN 17182:1992] auf das Einhalten der vorgeschriebenen Gehaltwerte des Kohlenstoffs und der Legierungselemente kontrolliert und korrigiert. Der Stahl wurde dem VARP -Verfahren, einem von der Friedrich Wilhelms-Hütte entwickelten Vakuum-Argon-Raffinations-Prozess, unterzogen. Mit dieser Sekundärmetallurgie ist es möglich, niedrigste Gehalte an Schwefel, Stickstoff, Wasserstoff und Sauerstoff einzustellen, wodurch ein besonders reines und gut schweissbares Material erzeugt werden kann.

\subsubsection{Wärmebehandlung}

Für die Knoten wurde wie erwähnt ein vergüteter Stahlguss gewählt. Beim Vergüten werden die mechanisch-technologischen Werte eingestellt, wobei der Stahl zuerst gehärtet und anschliessend angelassen wird. Beim Härten wird der austenitisierte Stahl mit der von der chemischen Zusammensetzung abhängigen kritischen oberen Abkühlgeschwindigkeit abgekühlt, damit das Härtegefüge Martensit entsteht, welches sich durch eine hohe Festigkeit auszeichnet, jedoch sehr spröde ist. Durch die schnelle Abkühlung wird insbesondere die Diffusion des Kohlenstoffs erschwert, was seine Zwangslösung zur Folge hat. Der gehärtete Stahl ist wegen seiner extremen Härte und Sprödigkeit nicht verwendbar. Bei der anschliessenden, Anlassen genannten, Wärmebehandlung diffundiert der zwangsgelöste Kohlenstoff wieder aus. Festigkeit und Härte nehmen ab, die Zähigkeit jedoch stark zu. Tab. 3.6 zeigt die Wärmebehandlungen der Stahlgussknoten mit den entsprechenden Temperaturen und Abkühlbedingungen. Die Abkühlbehandlung findet in einem grossen Wasserbad statt.

\begin{tabular}{lcc}
\hline & \multicolumn{2}{c}{ Wärmebehandlung } \\
\cline { 2 - 3 } & Härten & Anlassen \\
\hline Aufheizen & $8 \mathrm{~h}$ & $7 \mathrm{~h}$ \\
\hline Haltetemperatur & $950^{\circ} \mathrm{C}$ & $600^{\circ} \mathrm{C}$ \\
\hline Haltezeit & $8 \mathrm{~h}$ & $8 \mathrm{~h}$ \\
\hline Abkühlbehandlung & Wasserbad & $\begin{array}{l}\text { - von } 600^{\circ} \text { auf } 530^{\circ} \text { im Ofen } 530^{\circ} \text { auf } 300^{\circ} \text { im Wasserbad }{ }^{1} \\
- \text { von } 300^{\circ} \text { auf } 20^{\circ} \text { an der Luft }\end{array}$ \\
\hline
\end{tabular}

${ }^{1}$ zur Vermeidung der Anlassversprödung

Tab. 3.6: Wärmenachbehandlung der Stahlgussknoten 


\subsubsection{Gütewahl nach DIN 1690 Teil 2 und zerstörungsfreie Prüfung}

Für die Gussstücke wurden folgende Gütestufen gemäss der Norm [DIN 1690-2:1985] (vergleiche Abschnitt 2.2.3) gefordert:

- S2 V2 für die Anschweissenden

- S2 V3 für den Restbereich des Knotens

Die Einhaltung der Gütestufen wurde für die äussere Beschaffenheit mit dem Magnetpulververfahren und der Farbeindringprüfung, für die innere Beschaffenheit mittels Ultraschallprüfung kontrolliert. Es wurde mit dem Lieferanten vereinbart, dass Gussfehler, die nicht der gewählten Gütestufe entsprachen, nicht repariert sondern mit Lage und Grösse schriftlich dokumentiert werden. Aus diesem Grund wurden die Knoten der Probekörper A2, B0 und B2 mit der Gütestufe S4V3 für den Restbereich des Knotens geliefert (Tab. 3.7 auf Seite 38).

\subsubsection{Herstellung der Fachwerkträger}

\subsubsection{Schweissarbeiten}

Für die Serie A wurden die Stumpfschweissnähte ohne, für die Serie B mit Schweissbadsicherung ausgebildet, um den Einfluss der Schweissbadsicherung auf das Ermüdungsverhaltens der Schweissnähte zu untersuchen.

Die Fabrikation der Fachwerkträger wurde an zwei renommierte Stahlbaufirmen in der Schweiz vergeben. Beide Firmen sind im Besitz des Betriebsausweises S1 nach der Norm [SIA 161/1]. Die Fabrikationspläne für die Serie B werden als Beispiel im Anhang A abgedruckt.

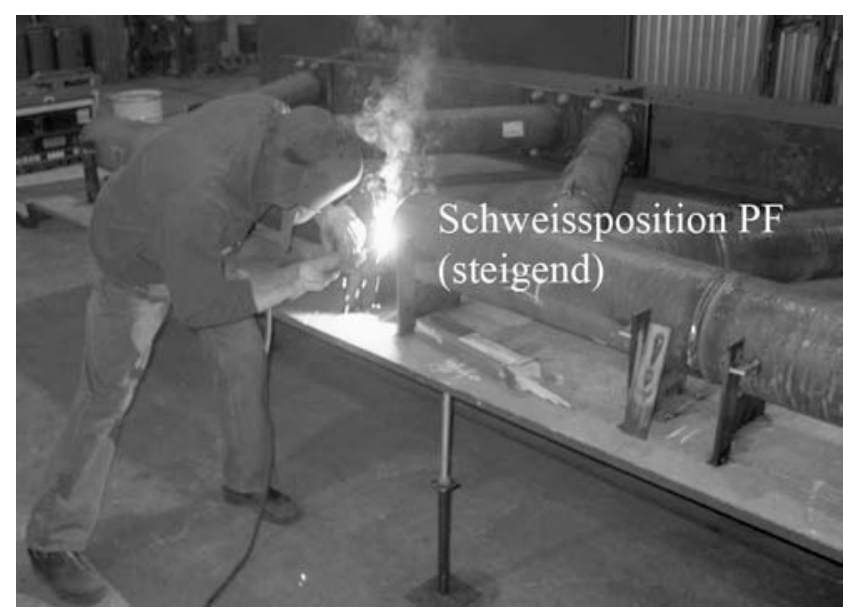

Fig. 3.4: Schweissen des Probekörpers

Für die Stumpfschweissnähte wurde die Bewertungsgruppe B nach [SIA 263] festgelegt. Die Anschweissenden waren ohne Vorwärmung schweissbar, jedoch mussten die Fugen schwitzwassertrocken sein und wurden daher auf $50-100^{\circ} \mathrm{C}$ trocken vorgewärmt. Zur Vermeidung von Wasserstoffrissen und um eine hohe Zähigkeit des Schweissbades zu garantieren, wurden Elektroden mit basischer Umhüllung des Typs Phönix 120K gewählt. Die Stumpfnähte wurden in der Position PF geschweisst. Die obere Hälfte der Stumpfschweissnaht wurde bei flach liegendem Probekörper ausgeführt (Fig. 3.4). Anschliessend wurde der Probekörper gedreht, um die zuvor nicht zugängliche Hälfte zu schweissen. 
In den nachfolgenden Abschnitten wird näher auf die Ausführung der Schweissnähte und die Fugenvorbereitung eingegangen.

\subsubsection{Fugenvorbereitung ohne Schweissbadsicherung (Serie A)}

Fig. 3.5 zeigt als Beispiel einer Schweissnahtvorbereitung ohne Schweissbadsicherung der Serie A schematisch das Schweissdetail zwischen Untergurtstutzen und Hohlprofil. Um ein gutes Durchschweissen mit dem Lichtbogenhandschweissverfahren (111) zu garantieren, müssen ein Wurzelabstand von $3 \mathrm{~mm}$ und eine Steghöhe von $2 \mathrm{~mm}$ eingehalten werden. Für das Ausfüllen der Schweissnaht mit dem Metall-Schutzgasschweissverfahren (135) ist ein Öffnungswinkel von $50^{\circ}$ notwendig. In Fig. 3.6 ist die Fabrikation einer Stumpfnaht dargestellt. Zunächst wurde der gesamte Probekörper mit Hilfe von gehefteten Blechen ausgerichtet (Fig. 3.6 a) und anschliessend mit Heftschweissnähten fixiert (Fig. 3.6 b).

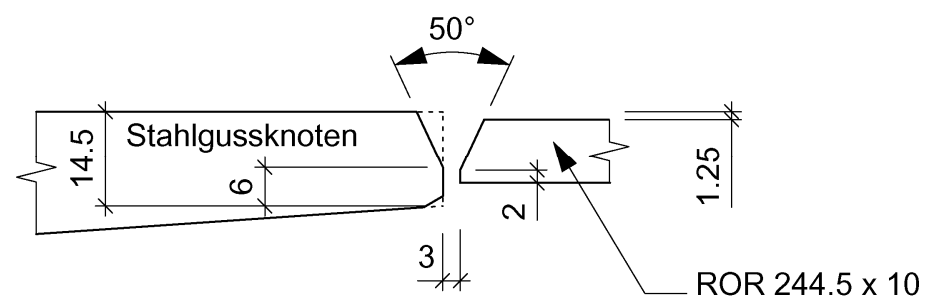

Fig. 3.5: Beispiel einer Schweissnahtvorbereitung ohne Schweissbadsicherung, Skizze Serie A

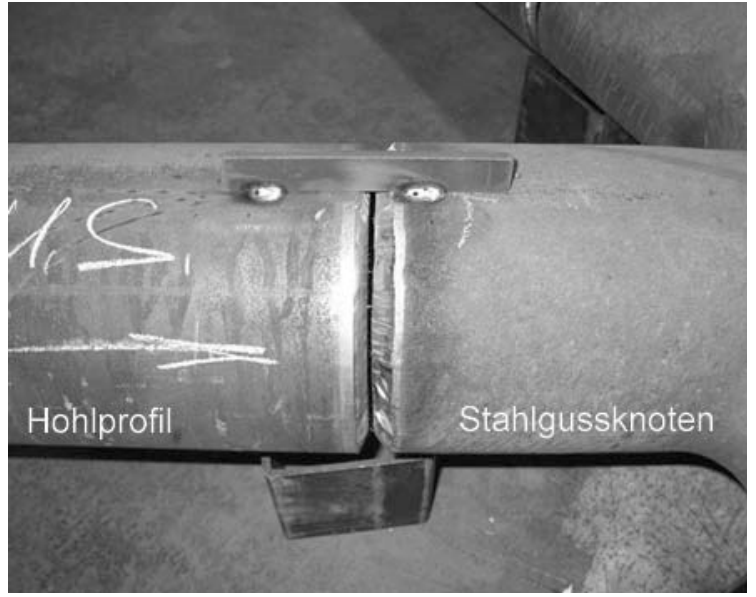

a) Ausrichten des Schweissnahtstumpfsstosses

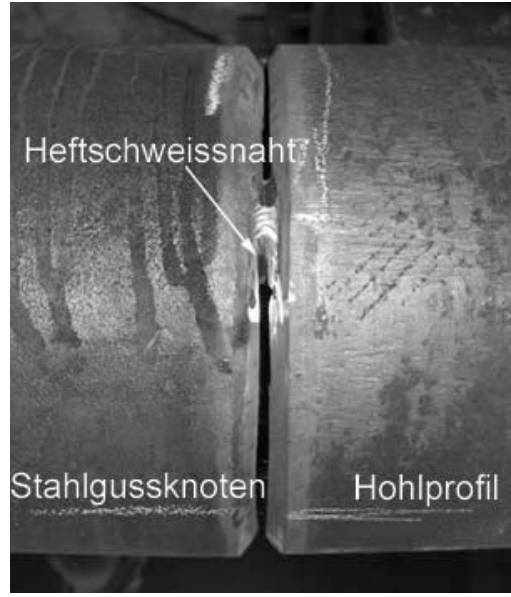

b) Geheftete Stumpfnaht

Fig. 3.6: Schweissnahtvorbereitung ohne Schweissbadsicherung, Fabrikation

\subsubsection{Fugenvorbereitung mit Schweissbadsicherung (Serie B)}

Fig. 3.7 zeigt als Beispiel einer Schweissnahtvorbereitung mit Schweissbadsicherung der Serie B schematisch das Schweissdetail zwischen Untergurtstutzen und Hohlprofil. Um die Toleranzen sehr gering zu halten, müssen die Innendurchmesser des Hohlprofils und des Anschweissendes des Knotenstutzens übereinstimmen. Die Innendurchmesser der gelieferten Hohlprofile wurden ausgemessen und die Anschweissenden der Knotenstutzen entsprechend angepasst. Die Badsicherung wurde aus einem Hohlprofil auf einen etwas kleineren Aussendurchmesser abgedreht, wobei die 
Wanddicke mindestens $5 \mathrm{~mm}$ betragen sollte, um ein Durchstechen der Elektrode zu vermeiden. Mit einer durchgehenden, verschliffenen und anschliessend zerstörungsfrei geprüften Schweissraupe (Fig. 3.8 a) wurde die Badsicherung an das Anschweissende angeschweisst. Nach dem Ausrichten des Probekörpers wurden die Stumpfnähte geheftet (Fig. 3.8 b). Der Abstand von $5 \mathrm{~mm}$ in der Schweissnahtwurzel bietet genügend Platz für die zweite Schweissraupe der ersten Lage. Für das Ausfüllen der Schweissnaht mit dem Lichtbogenhandschweissverfahren (111) wurde ein Öffnungswinkel von $50^{\circ}$ gewählt.

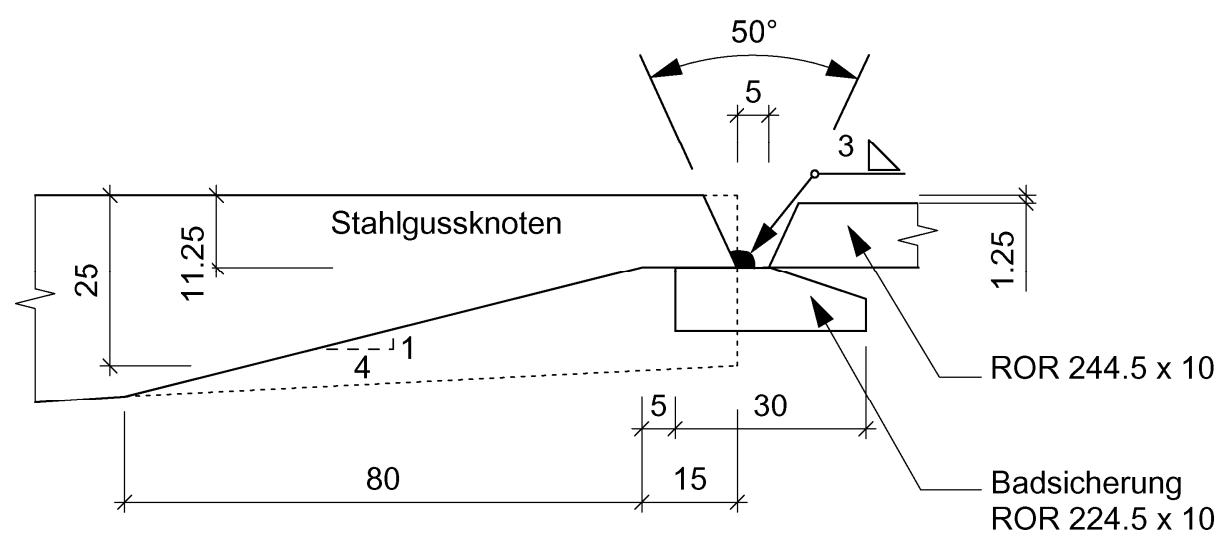

Fig. 3.7: Beispiel einer Schweissnahtvorbereitung mit Schweissbadsicherung, Skizze Serie B

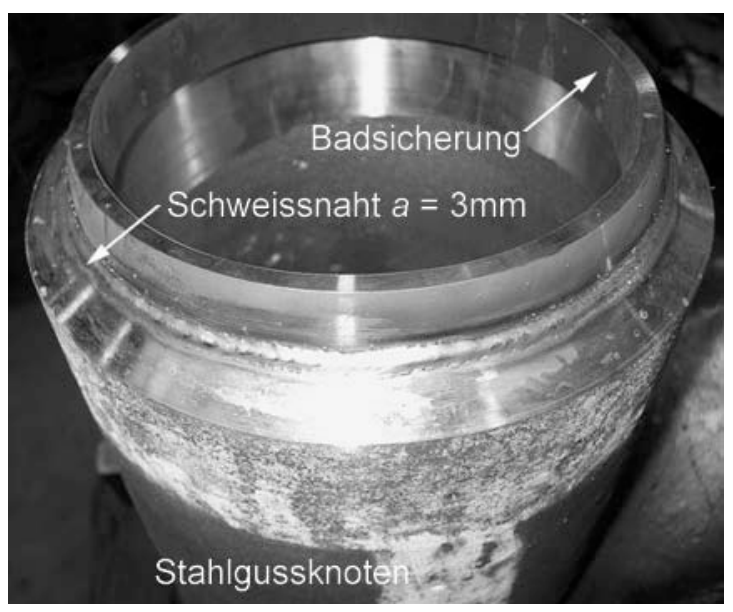

a) Badsicherung am Knotenstutzen, noch ungeschliffen

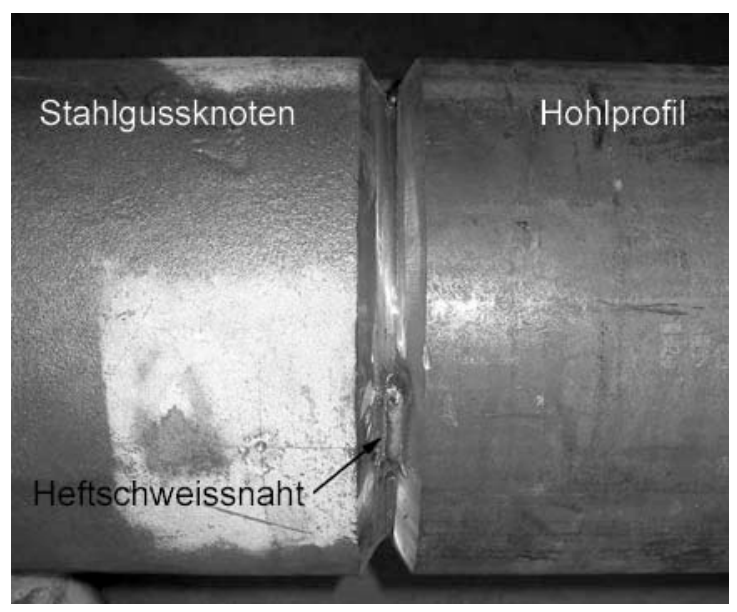

b) Geheftete Stumpfnaht, noch ungeschliffen

Fig. 3.8: Schweissnahtvorbereitung mit Schweissbadsicherung, Fabrikation

\subsection{VERSUCHSDURCHFÜHRUNG}

\subsubsection{Versuchsprogramm}

Anhand der zu untersuchenden Parameter wurde ein aus zwei Versuchsserien bestehendes Versuchsprogramm ausgearbeitet (Tab. 3.7). In der Serie A wurde der Einfluss der Stahlgussgüte, der Ausbildung der Schweissnähte ohne Badsicherung und der aussermittigen Anschlüsse der Hohlprofile (bedingt durch das Dickenverhältnis $\tau_{1} / \tau_{2}$ ) auf das Ermüdungsverhalten der Gussstücke 
untersucht. Die Schweissnähte des Probekörpers der Voruntersuchung waren teilweise stark fehlerbehaftet und ungenügend durchgeschweisst. Nach dem Auswerten der Versuchsergebnisse der Serie A wurden die Parameter für die Serie B festgelegt.

Im Wesentlichen wurden zwei Parameter für das Ermüdungsverhalten von Stahlgussknoten identifiziert: die Ausbildung der Schweissnähte, insbesondere die Qualität der Schweissnahtwurzel, und die Zusatzbelastung des Gussknotens und der Schweissnähte aus Sekundärbiegemomenten. Daher wird in der Serie B der Einfluss der unterschiedlichen Steifigkeiten des Gussknotens und der anschliessenden Hohlprofile auf die Spannungsverteilung im Knoten und in den Schweissnähten untersucht. Dieser Steifigkeitssprung wird sowohl durch eine kompaktere Ausbildung der Gussknoten (kürzere Knotenstutzen und grössere Wanddicken) als auch durch die Wahl grösserer Wanddicken der Hohlprofile variiert. Die Dickenverhältnisse $\tau_{1}$ und $\tau_{2}$ beziehen sich auf die noch unbearbeitete Wanddicke des Stutzens, ohne den Steigungswinkel von 1:4 zu berücksichtigen (vergleiche Fig. 3.7). Die Schweissnähte wurden für die Serie B quasi ohne Wanddickensprung und mit einer Schweissbadsicherung ausgebildet, um die Qualität der Schweissnahtwurzel und damit die Ermüdungsfestigkeit der Schweissnähte zu verbessern.

\begin{tabular}{|c|c|c|c|c|c|c|c|c|}
\hline \multirow[t]{2}{*}{$\begin{array}{l}\text { Serie } \\
\text { Probekör- } \\
\text { per Nr. }\end{array}$} & \multicolumn{2}{|c|}{$\begin{array}{l}\text { Stahlgussgüte } \\
\text { (DIN 1690-2) }\end{array}$} & \multicolumn{2}{|c|}{$\begin{array}{c}\text { Abmessungen der Rund- } \\
\text { hohlprofile } \\
\text { (Durchmesser / Dicke) }\end{array}$} & \multirow[t]{2}{*}{$\begin{array}{c}\text { Schweiss- } \\
\text { nahtart }\end{array}$} & \multicolumn{2}{|c|}{ Steifigkeitsparameter } & \multirow{2}{*}{$\begin{array}{c}\text { Diagona- } \\
\text { lenwinke } \\
\theta \\
{\left[^{\circ}\right]}\end{array}$} \\
\hline & $\begin{array}{l}\text { An- } \\
\text { schweiss- } \\
\text { enden }\end{array}$ & $\begin{array}{c}\text { Restbe- } \\
\text { reich }\end{array}$ & $\begin{array}{l}\text { Diagonale } \\
\text { [mm }]\end{array}$ & Untergurt & & $\begin{array}{c}\text { Stutzenlänge } \\
\text { (Untergurt / } \\
\text { Diagonale) } \\
{[\mathrm{mm}]} \\
\end{array}$ & $\begin{array}{l}\text { Dickenver- } \\
\text { hältnis } \\
\tau_{1} / \tau_{2} \\
{[-]} \\
\end{array}$ & \\
\hline Vorversuch & S2V2 & S3V3 & $193.7 / 8$ & $244.5 / 10$ & $\mathrm{FP}^{1}$ & $1000 / 480$ & $1.7 / 1.4$ & 60 \\
\hline A1 & $\mathrm{S} 2 \mathrm{~V} 2$ & S3V3 & $193.7 / 8$ & $244.5 / 10$ & $\mathrm{FP}$ & $970 / 465$ & $1.7 / 1.4$ & 60 \\
\hline A2 & S2V2 & S4V3 & $193.7 / 8$ & $244.5 / 10$ & FP & $970 / 465$ & $1.7 / 1.4$ & 60 \\
\hline B0 & S2V2 & S4V3 & $193.7 / 8$ & $244.5 / 10$ & $\mathrm{FP} / \mathrm{BR}$ & $800 / 370$ & $2.6 / 2.5$ & 60 \\
\hline B1 & S2V2 & S3V3 & $193.7 / 12.5$ & $244.5 / 17.5$ & $\mathrm{FP} / \mathrm{BR}$ & $800 / 370$ & $1.7 / 1.4$ & 60 \\
\hline B2 & $\mathrm{S} 2 \mathrm{~V} 2$ & S4V3 & $193.7 / 12.5$ & $244.5 / 17.5$ & $\mathrm{FP} / \mathrm{BR}$ & $800 / 370$ & $1.7 / 1.4$ & 60 \\
\hline
\end{tabular}

FP: durchgeschweisste Stumpfnaht (full penetration); ${ }^{1}$ fehlerbehaftete Stumpfnaht

BR: Badsicherung (backing ring)

$\tau_{1}=$ Wanddickenverhältnis Stutzen- zu Hohlprofildicke der Diagonale (Serie B: Stutzendicke ohne Steigungswinkel 1:4)

$\tau_{2}=$ Wanddickenverhältnis Stutzen- zu Hohlprofildicke des Untergurts (Serie B: idem wie bei $\tau_{1}$ )

Tab. 3.7: Versuchsprogramm

\subsubsection{Versuchsaufbau}

Fig. 3.9 zeigt den Versuchsaufbau und die Krafteinleitung auf dem Obergurt. Die Auflager an beiden Enden des Fachwerkträgers werden durch Betonblöcke realisiert. Um die erforderliche Höhe der Auflager zu erreichen, werden zusätzlich Hohlprofile unterlegt. Der Fachwerkträger ist einfach gelagert. Da er auf den Hohlprofilen aufliegt und nur die seitliche Verschiebung verhindert wird, ist die Längsverschiebung des Fachwerkträgers nahezu frei. Die Krafteinleitung geschieht mit Hilfe von zwei Hydraulikkolben, die mittels eines kurzen Profilträgers die Kraft mittig aufbringen. 


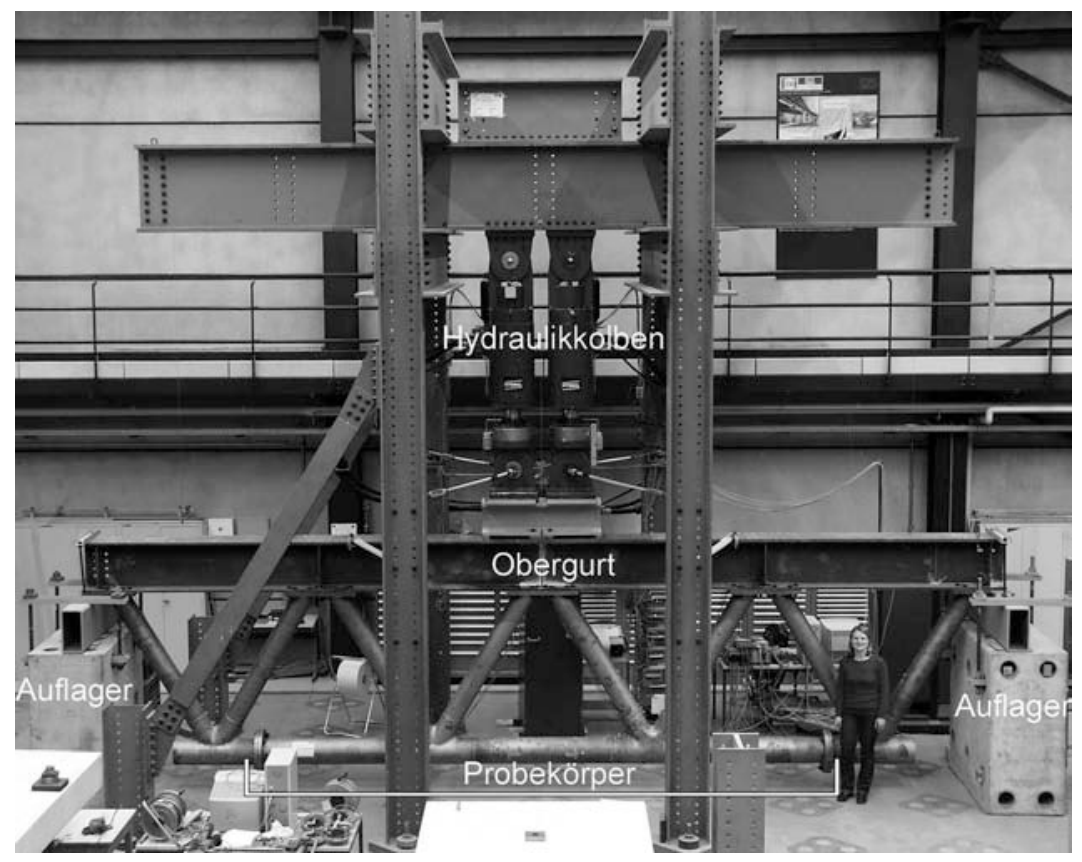

Fig. 3.9: Versuchsaufbau: Probekörper und Versuchsrahmen

\subsubsection{Statische Versuche}

Vor Beginn eines Ermüdungsversuchs wird der Probekörper zuerst statisch belastet, um das linear elastische Verhalten und das Messsystem zu überprüfen. Dabei wird die Last auf drei Niveaus zwischen der minimalen Ermüdungslast $Q_{\min }=100 \mathrm{kN}$ und der maximalen Ermüdungslast $Q_{\max }=1000 \mathrm{kN}$ hoch- und zurückgefahren. Auf jedem Lastniveau werden drei Messungen mit den auf dem Fachwerkträger aufgebrachten Dehnmessstreifen (DMS) durchgeführt. Die Auswertung der Messungen zeigt anschliessend, ob die DMS stabil und ohne grosse Messabweichungen arbeiten und ob die Dehnungen im Fachwerkträger linear vom Lastniveau abhängen. Nach den ersten ca. 20'000 Lastwechseln wird ein zweiter statischer Versuch durchgeführt.

Die Dehnungen in den Stahlgussknoten und auf den Hohlprofilen waren durchwegs proportional zum Lastniveau. Bei den Dehnmessstreifen in der Nähe eines Schweissnahtfusses wurde oft erst ab dem zweiten statischen Versuch ein lineares Verhalten beobachtet. Dies lässt auf das Vorhandensein von durch den Fabrikationsprozess entstandenen Schweisseigenspannungen schliessen.

Statische Versuche werden auch während eines Ermüdungsversuchs in regelmässigen Abständen durchgeführt. Dazu wird der Ermüdungsversuch angehalten und der Probekörper wird mit den drei Lastniveaus belastet, während Dehnungsmessungen durchgeführt werden. Änderungen der gemessenen Dehnungen können Hinweise auf mögliche Ermüdungsrisse in den Schweissnähten geben, insbesondere wenn der Ermüdungsriss von der Wurzelseite der Schweissnaht ausgeht und noch nicht visuell erkennbar ist.

\subsubsection{Ermüdungsversuche}

Nach dem ersten statischen Versuch wird für jeden Probekörper ein Ermüdungsversuch durchgeführt. Dabei wird der Rissfortschrittsverlauf beobachtet und die auf den Probekörper aufgebrachten Lastwechsel bis zum Bruch werden gezählt. 
Für den Ermüdungsversuch wird mit Hilfe von zwei Hydraulikkolben eine variable Last mit konstanter Schwingbreite von $\Delta Q=900 \mathrm{kN}$, einem Lastverhältnis von $Q_{\min } / Q_{\max }=0.1$ und einem sinusförmigen Verlauf mit einer Frequenz von $1.25 \mathrm{~Hz}$ auf den Fachwerkträger aufgebracht. Die zwei Hydraulikkolben arbeiten synchron. Um die Schwingbreite der variablen Last im Verlauf des Ermüdungsversuchs konstant zu halten, orientiert sich die Steuerung der Hydraulikkolben unabhängig von der Durchbiegung des Trägers an der aufzubringenden Last. Aus Sicherheitsgründen werden die Obergrenzen der Last und der Trägerdurchbiegung eng begrenzt. Ein Überschreiten dieser Grenzen führt zum sofortigen Versuchsstopp.

Die Schweissnähte werden regelmässig mit Oberflächenrissprüfungen (Magnetpulver- und Farbeindringprüfung) und statischen Dehnungsmessungen auf Rissentstehung und Risswachstum untersucht. Die Stahlgussknoten werden ebenfalls visuell beobachtet und durch Dehnungsmessungen auf Veränderungen ihres mechanischen Verhaltens hin überprüft. Während den Ermüdungsversuchen wurden jedoch nur Ermüdungsrisse in den Schweissnähten zwischen den Knotenstutzen und den anschliessenden Hohlprofilen beobachtet. Es wird festgelegt, dass eine Schweissnaht als gebrochen gilt, wenn der Durchriss mehr als die Hälfte des Hohlprofilumfangs beträgt, da ab diesem Rissstadium die Querschnittssteifigkeit rasch abnimmt.

Je nachdem in welcher Schweissnaht der erste Ermüdungsriss auftritt, wird diese repariert und der Ermüdungsversuch fortgesetzt. Bei den meisten Versuchskörpern wurde nur eine Reparatur durchgeführt und der Versuch nach dem Auftreten eines weiteren Ermüdungsrisses bis zum Bruch gefahren und dann abgebrochen.

Für die Schweissnahtreparatur muss der Probekörper aus dem Versuchsrahmen ausgebaut werden, um die Zugänglichkeit für die Schweissarbeiten zu gewährleisten. Da es sich beim Ermüdungsriss um einen durch die Wanddicke durchgehenden Riss handelt, wird auf ein Reparaturschweissen in der Schweissnaht verzichtet. Dazu müsste der Riss durch Ausschleifen der Schweissnaht entfernt und anschliessend diese Stelle wieder zugeschweisst werden, was auf Grund der verhinderten Schrumpfungen des Schweissnahtguts zu erheblichen Verformungen und Eigenspannungen führen würde. Ausserdem sollte die Bruchfläche unversehrt bleiben, damit sie nach Beendigung des Versuchs freigelegt und visuell beurteilt werden kann. Der Riss wird daher mit zwei Halbschalen abgedeckt, die zuerst an den Längskanten zusammengeschweisst und anschliessend an den beiden Enden auf die Aussenseite des Untergurts geschweisst werden. Um einen weichen Spannungsfluss in der Reparaturschweissnaht zwischen der Halbschale und dem Untergurt zu ermöglichen, werden die Endkanten der Halbschalen nach innen mit einem Winkel von $50^{\circ}$ abgekantet, der mit einer Schweissraupe aufgefüllt wird. Die Längskanten sind in einem Winkel von $25^{\circ}$ abgekantet. In Fig. 3.10 wird eine Reparaturstelle im Untergurt dargestellt. Die Oberfläche der Schweissnaht ist ausgeschliffen, um Einbrandkerben zu vermeiden.

Die vorgeschlagene Schweissnahtreparatur ist relativ kosten- und zeitaufwendig. Ein Schweisser benötigt durchschnittlich 110 Minuten pro Schweissnahtraupe und insgesamt 15 Stunden für die Reparatur mit Halbschalen, deren Innenkanten abgekantet sind. Dies ergibt einen Gesamtkostenaufwand von ungefähr 1250 CHF pro Reparatur (im Jahr 2003). 


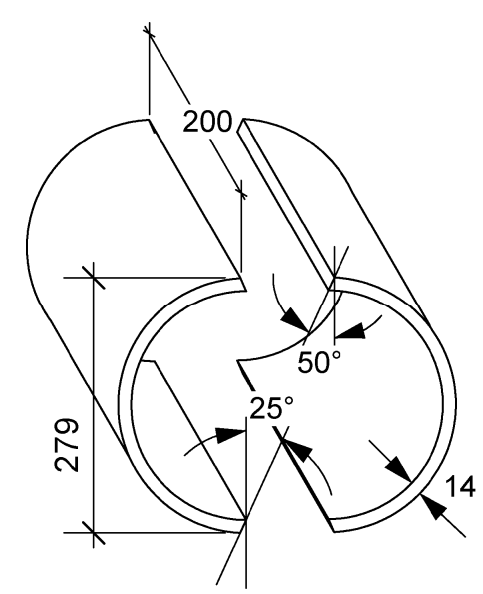

a) Halbschalen

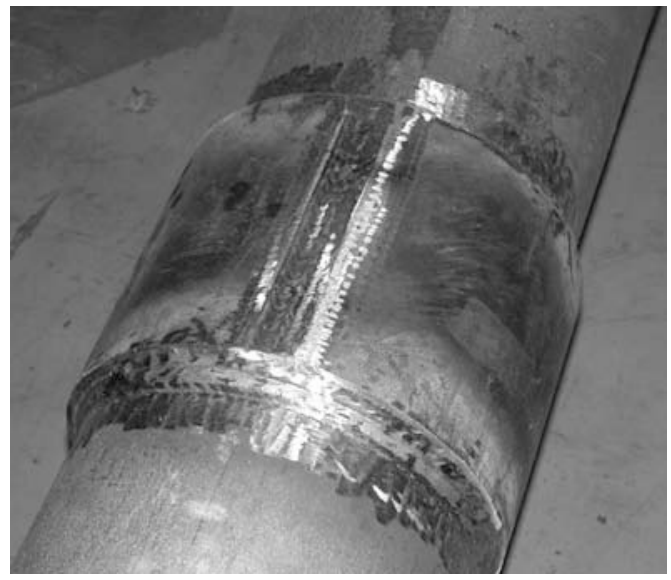

b) Abgeschliffene Schweissnähte der Reparatur

Fig. 3.10: Beispiel einer Schweissnahtreparatur im Untergurt

\subsection{MESSUNGEN}

\subsubsection{Messungen unter statischer Last}

Der vorliegenden Arbeit vorangehende experimentelle Untersuchungen zum Ermüdungsverhalten von geschweissten Knoten [Schumacher 2003] haben gezeigt, dass die Steuerung der Hydraulikkolben korrekte Werte für die aufgebrachte Last und für die Auslenkung der Kolben (= Durchbiegung des Trägers) liefert. Aus diesem Grund wird hier auf zusätzliche externe Messungen verzichtet.

Während der statischen Versuche werden die Oberflächendehnungen auf den Diagonalen und dem Untergurt der Probekörper gemessen. Fig. 3.11 zeigt die Anordnung der Dehnmessstreifen (DMS) im Fachwerkträger. Die DMS sind generell parallel zur Profilachse ausgerichtet, um die Dehnungen aus Normalkraft $\varepsilon_{\mathrm{ax}}$ und Biegemoment $\varepsilon_{\mathrm{b}}$ in den Fachwerkstäben zu messen. Damit im Untergurt Sekundärbiegemomente senkrecht zur Trägerebene überprüft werden können, werden in der Mitte des Untergurts zwei Querschnitte mit je vier DMS versehen. Der Abstand zwischen der Schweissnaht und den DMS von $1.5 \mathrm{D}$ bzw. $1.5 d$ wird eingehalten, um nicht lokale Sekundärbiegemomente durch Steifigkeitssprünge und/oder exzentrische Anschlüsse in die Messungen mit einzuschliessen.

Die Diagonalen und der Untergurt werden mit einachsigen, $10 \mathrm{~mm}$ langen DMS (elektrische Widerstandsmessung) versehen. Da in den aussermittigen Anschlüssen der Hohlprofile an die Stutzen der Gussknoten erhöhte Spannungen am Fuss der Schweissnaht zu erwarten sind, werden je nach Wanddicke des anschliessenden Hohlprofils 1.5 bis $4.0 \mathrm{~mm}$ lange DMS am Schweissnahtfuss sowohl auf der Hohlprofilseite als auch auf der Seite des Gussknotens angebracht. 


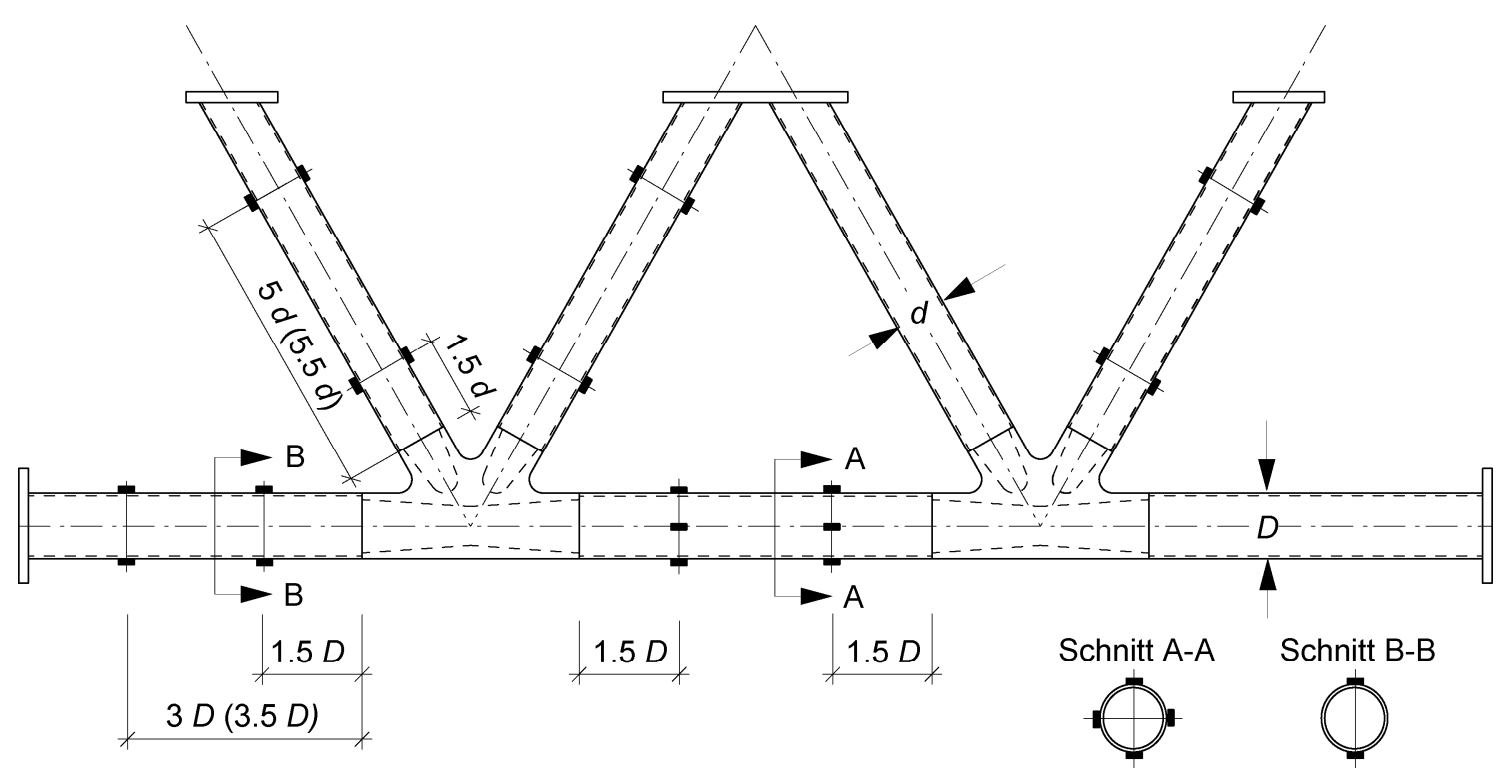

Fig. 3.11: Anordnung der Dehnmessstreifen auf den Hohlprofilen des Probekörpers der Serie A; Angaben in Klammern für Serie B

Die Stahlgussknoten sind in den Bereichen möglicher Spannungskonzentrationen - in den Ausrundungen zwischen den beiden Diagonalen und zwischen der Druckdiagonale und dem Untergurt - sowie auf der Untergurtseite mit DMS ausgestattet. Die DMS sind ebenfalls in der Längsebene des Probekörpers parallel zur Stutzenachse ausgerichtet, einachsig und $3.0 \mathrm{~mm}$ lang. Fig. 3.12 zeigt als Beispiel die Anordnung der Dehnmessstreifen für den Knoten Nr. 19 des Probekörpers A1.
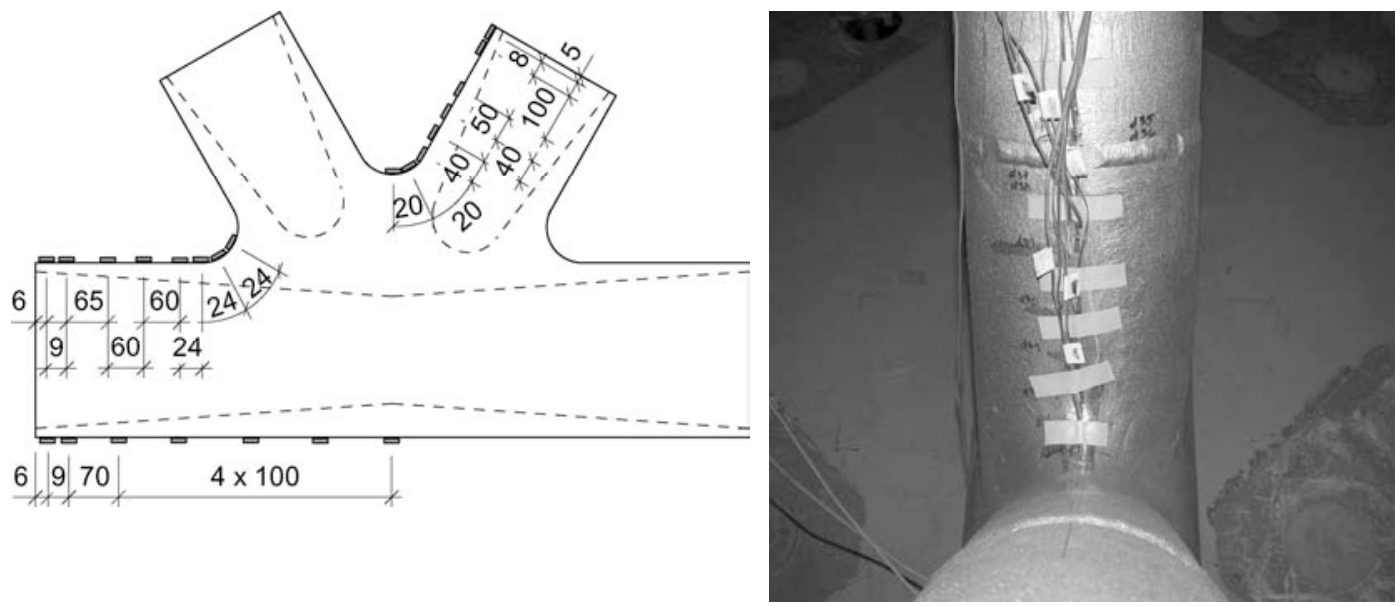

a) Anordnung der Dehnmessstreifen

b) Aufsicht auf eine Zugdiagonale

Fig. 3.12: Dehnmessstreifen im Stahlgussknoten Nr. 19, Probekörper A1

\subsubsection{Messungen unter Ermüdungslast}

Als ergänzende Kontrolle zu den Messwerten unter statischer Last werden an einigen Dehnmessstreifen Messungen unter Ermüdungslast durchgeführt. Die DMS sind zur Datenerfassung an ein digitales Mehrkanalmesssystem von HBM mit 8 Kanälen (Spider 8) angeschlossen. Die limitierte Anzahl Kanäle ermöglicht neben zwei Kanälen für die Hydraulikkolben den Anschluss von sechs 
DMS. Mit dem Spider 8 können je nach Bedarf in regelmässigen Abständen kontinuierlich während einer bestimmten Zeitspanne Messwerte aufgenommen werden. Der Vergleich der Messwerte zeigt, dass der Maximalwert der Dehnungsmessungen unter Ermüdungslast nur unwesentlich von demjenigen unter statischer Last abweicht. Damit können für die Erfassung der Spannungsschwingbreiten an den Messstellen im Probekörper die Messwerte unter statischer Last verwendet werden.

Es wurden ausschliesslich Ermüdungsrisse in den Schweissnähten zwischen den Knotenstutzen und den anschliessenden Hohlprofilen beobachtet. Da die Risse immer von der Wurzelseite der Schweissnaht ausgingen, konnten sie visuell erst bei Wanddurchbruch beobachtet werden. Am Schweissnahtfuss befindliche DMS zeigten bereits vor dem Durchbruch erhöhte Dehnungen an, die auf einen wachsenden Riss schliessen liessen. Nach dem Wanddurchbruch konnte ein Riss sehr gut visuell beobachtet werden. Da Risse ab dem Wanddurchbruch sehr schnell wachsen, stellte es sich als schwierig heraus, die genaue Anzahl Lastwechsel bei Durchbruch festzustellen.

Zum Erfassen des Risswachstums wird die Länge des Risses auf der Aussenseite der Schweissnaht in regelmässigen Abständen während des Ermüdungsversuchs mit dem Magnetpulververfahren genau vermessen und zusammen mit der Anzahl Lastwechsel vermerkt. Zur Erstellung der Wöhlerkurven wird die Anzahl Lastwechsel beim Bruch und die an der Rissstelle vorherrschende Spannungsschwingbreite verwendet.

\subsection{VERSUCHSERGEBNISSE UND AUSWERTUNG}

\subsubsection{Statische Versuche}

Auf Grund der hohen Steifigkeit der Stahlgussknoten werden die Diagonalen und der Untergurt nicht nur durch Axialkräfte, sondern auch durch Sekundärmomente, die entlang der Stabachse linear variieren, belastet. Die Schnittkräfte werden aus den Dehnungsmesswerten in den Fachwerkstäben ermittelt. Da für die weiteren Untersuchungen die Belastung der Schweissnähte und der Stahlgussknoten erforderlich ist, müssen die am Ende der Knotenstutzen angreifenden Sekundärmomente bestimmt werden. Dazu werden die an den DMS gemessenen Dehnungen, wie in Fig. 3.13 skizziert, an die Enden der Knotenstutzen extrapoliert. Mit zwei DMS kann indessen nicht nachgewiesen werden, dass die Sekundärmomente linear variieren. Dies wird mit Hilfe eines numerischen Modells des Probekörpers (siehe Abschnitt 4.2) überprüft und bestätigt.

Tab. 3.8 fasst die Durchschnittswerte für die Dehnungen in den Fachwerkstäben des jeweiligen Probekörpers unter der Last $Q_{\max }=1000 \mathrm{kN}$ zusammen. Die Dehnungen sind nach verursachenden Schnittkräften getrennt aufgeführt: $\varepsilon_{\mathrm{ax}}$ bezeichnet die Dehnung infolge der Axialkraft, $\varepsilon_{\mathrm{b}}$ diejenige infolge des Sekundärbiegemoments. Die Nominaldehnung $\varepsilon_{\text {nom }}$ ergibt sich aus der Superposition von $\varepsilon_{\mathrm{ax}}$ und $\varepsilon_{\mathrm{b}}$. Die Werte beziehen sich auf die in Fig. 3.13 mittels Pfeilen markierte Profilseite. 


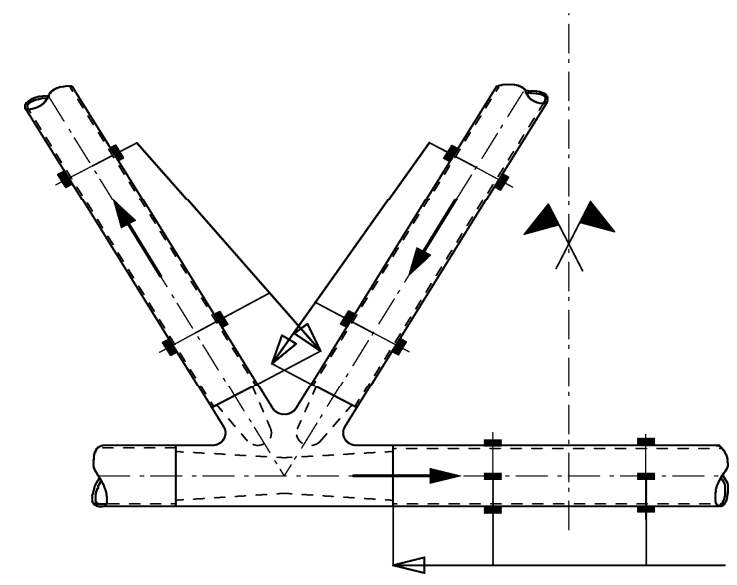

Fig. 3.13: Extrapolation der Messwerte an die Enden der Knotenstutzen

\begin{tabular}{|c|c|c|c|c|}
\hline \multirow[t]{2}{*}{ Fachwerkstab } & Dehnungen & (1) & (2) & (3) \\
\hline & & $\begin{array}{c}\text { Serie A } \\
(\mathrm{A} 1+\mathrm{A} 2) \\
{[\mu \mathrm{m} / \mathrm{m}]}\end{array}$ & $\begin{array}{c}\text { Serie B } \\
(\mathrm{B} 0) \\
{[\mu \mathrm{m} / \mathrm{m}]}\end{array}$ & $\begin{array}{c}\text { Serie B } \\
(\mathrm{B} 1+\mathrm{B} 2) \\
{[\mu \mathrm{m} / \mathrm{m}]}\end{array}$ \\
\hline \multirow{4}{*}{ Zugdiagonale } & $\varepsilon_{\mathrm{ax}}$ & 364 & 357 & 267 \\
\hline & $\varepsilon_{\mathrm{b}}$ & 214 & 57 & 126 \\
\hline & $\varepsilon_{\text {nom }}$ & 578 & 414 & 393 \\
\hline & $\varepsilon_{\mathrm{b}} / \varepsilon_{\text {nom }}$ & 0.370 & 0.138 & 0.321 \\
\hline \multirow{4}{*}{ Druckdiagonale } & $\varepsilon_{\mathrm{ax}}$ & -395 & -371 & -274 \\
\hline & $\varepsilon_{\mathrm{b}}$ & 67 & 29 & 10 \\
\hline & $\varepsilon_{\text {nom }}$ & -462 & -400 & -284 \\
\hline & $\varepsilon_{\mathrm{b}} / \varepsilon_{\text {nom }}$ & 0.145 & 0.073 & 0.035 \\
\hline \multirow{4}{*}{ Untergurt } & $\varepsilon_{\mathrm{ax}}$ & 612 & 600 & 376 \\
\hline & $\varepsilon_{\mathrm{b}}$ & 119 & 105 & 74 \\
\hline & $\varepsilon_{\text {nom }}$ & 731 & 705 & 450 \\
\hline & $\varepsilon_{\mathrm{b}} / \varepsilon_{\text {nom }}$ & 0.163 & 0.149 & 0.164 \\
\hline
\end{tabular}

Tab. 3.8: Dehnungen, extrapolierte Messwerte

Im Gegensatz zur Zugdiagonalen ist der Anteil von $\varepsilon_{\mathrm{b}}$ an den Nominaldehnungen $\varepsilon_{\text {nom }}$ für die Druckdiagonale und den Untergurt generell relativ niedrig. Dies liegt unter anderem daran, dass unter der mittig angesetzten Einzellast die durch die Querkraft induzierten Sekundärbiegemomente von der Trägermitte zum Auflager hin in den Fachwerkstäben zunehmen. Da die Zugdiagonale dem Auflager näher liegt, ist das Sekundärbiegemoment grösser als in der direkt unter der Einzellast liegenden Druckdiagonale und im Untergurt.

Die Verkürzung der Stutzenlängen hat zwei Auswirkungen. Einerseits verschiebt sich die Schweissnaht gegen den Achsenschnittpunkt zu, also in Richtung höherer Sekundärbiegemomente. Andererseits werden die Rundhohlprofile länger, ihre Biegesteifigkeit und damit die Sekundärbiegemomente nehmen ab. Der Vergleich von Spalte 1 und 2 in Tab. 3.8 zeigt, dass in den Diagonalen der zweite Effekt überwiegt, die Sekundärbiegemomente nehmen insgesamt ab. Im Untergurt heben sich die Einflüsse gegenseitig auf. Die Reduktion der Steifigkeit der Rundhohlprofile bei gleich bleibender Stutzenlänge führt erwartungsgemäss zu einer weiteren Abnahme der Sekundärbiegemo- 
mente (vergleiche Spalte 3 mit Spalte 2), ausser in der Druckdiagonale. Dies dürfte auf Messfehler zurückzuführen sein, die umso stärker ins Gewicht fallen, weil die Sekundärbiegemomente in der Druckdiagonale sehr klein sind. Änderungen der relativen Steifigkeit zwischen den Stahlgussknoten und den anschliessenden Hohlprofilen haben demnach einen grossen Einfluss auf die Beanspruchung der Schweissnähte der Diagonalen.

Auf Grund des im Wesentlichen einaxialen Spannungszustands können die Messdaten mit dem Hookschen Gesetz $\sigma=E \cdot \varepsilon$ in Hauptspannungen umgerechnet werden.

In den nachstehenden Figuren werden die Dehnungen in verschiedenen Bereichen im Stahlgussknoten dargestellt. Da sich die Knotengeometrie der Serie A und B hauptsächlich in der Stutzenlänge und der Wanddicke unterscheidet, ist der Dehnungsverlauf in den beiden Knotentypen unter gleicher Belastung vergleichbar. Entsprechend werden hier als Beispiel nur die Dehnungsverläufe der Knoten der Serie A gezeigt.

In Fig. 3.14 ist der Dehnungsverlauf auf der Innenseite der beiden Diagonalstutzen abgebildet. Auf der Abszisse wird der Abstand vom Scheitelpunkt zwischen den Diagonalstutzen bis zum Stutzenrand abgetragen, die Ordinate gibt die Nominaldehnungen wieder. Der Dehnungsverlauf weist eine Spitze in der Ausrundung auf der Seite der Zugdiagonalen auf. Auf der Seite der Druckdiagonalen reicht die Anzahl von DMS nicht aus, um dies darzustellen. Anschliessend nimmt der Dehnungsverlauf bei Annäherung an den Stutzenrand wegen der linearen Wanddickenabnahme quadratisch zu. Die Dehnungsverläufe der untersuchten Knoten zeigen keine grossen Abweichungen.

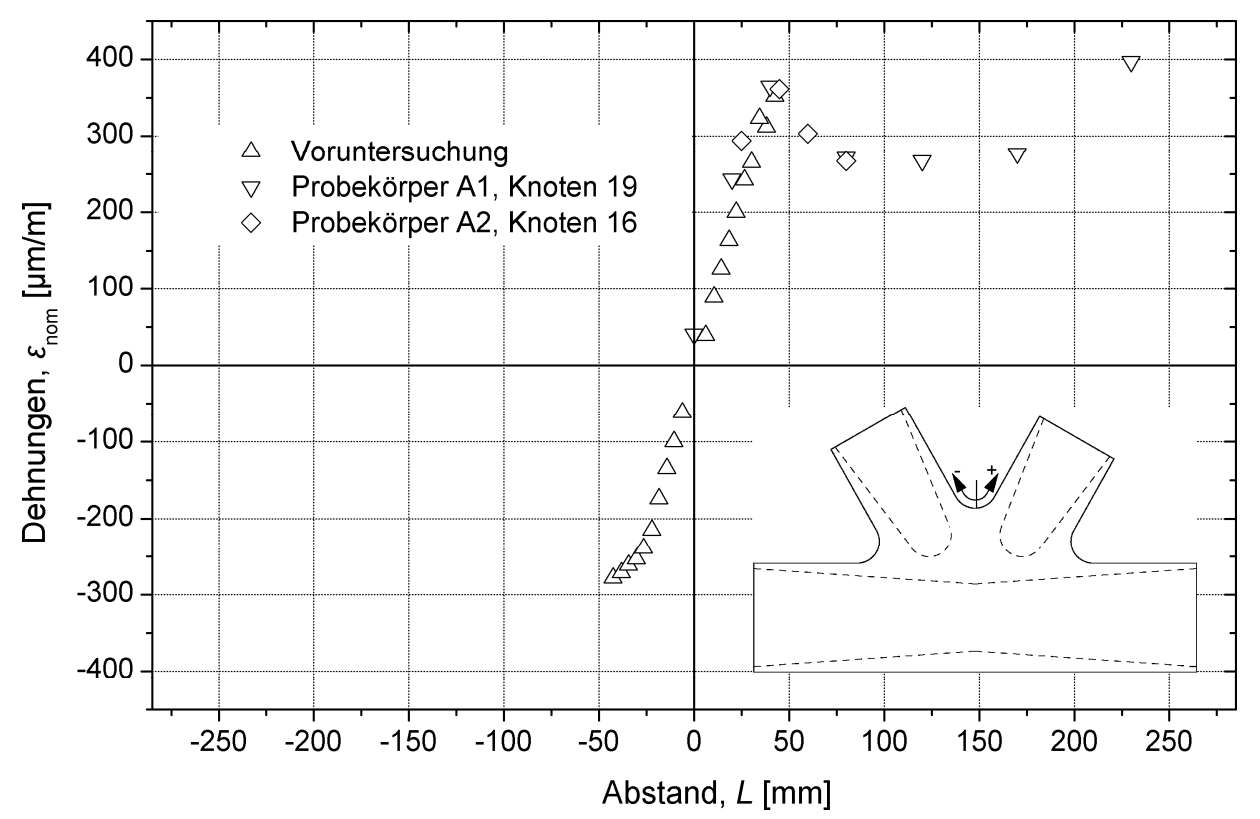

Fig. 3.14: Dehnungen auf der Innenseite der Diagonalstutzen, Serie A

In Fig. 3.15 wird der Dehnungsverlauf auf der Unterseite des Untergurtstutzens in Richtung der Mitte des Probekörpers (negativer Abstand) dargestellt. Da sich keine geometrischen Besonderheiten auf der Unterseite befinden, ist hier keine Dehnungsspitze vorzufinden. Die Dehnungen nehmen gegen das Stutzenende hin quadratisch zu, da die Wanddicke auf der Unterseite linear abnimmt. 
Fig. 3.16 zeigt den Dehnungsverlauf der Oberseite des Untergurtstutzens ab der Mitte der Ausrundung zwischen der Druckdiagonalen und dem Untergurtstutzen. Hier ist eine sehr schwache Dehnungsspitze in der Ausrundung mit anschliessendem quadratischen Anstieg der Dehnungszunahme zu erkennen.

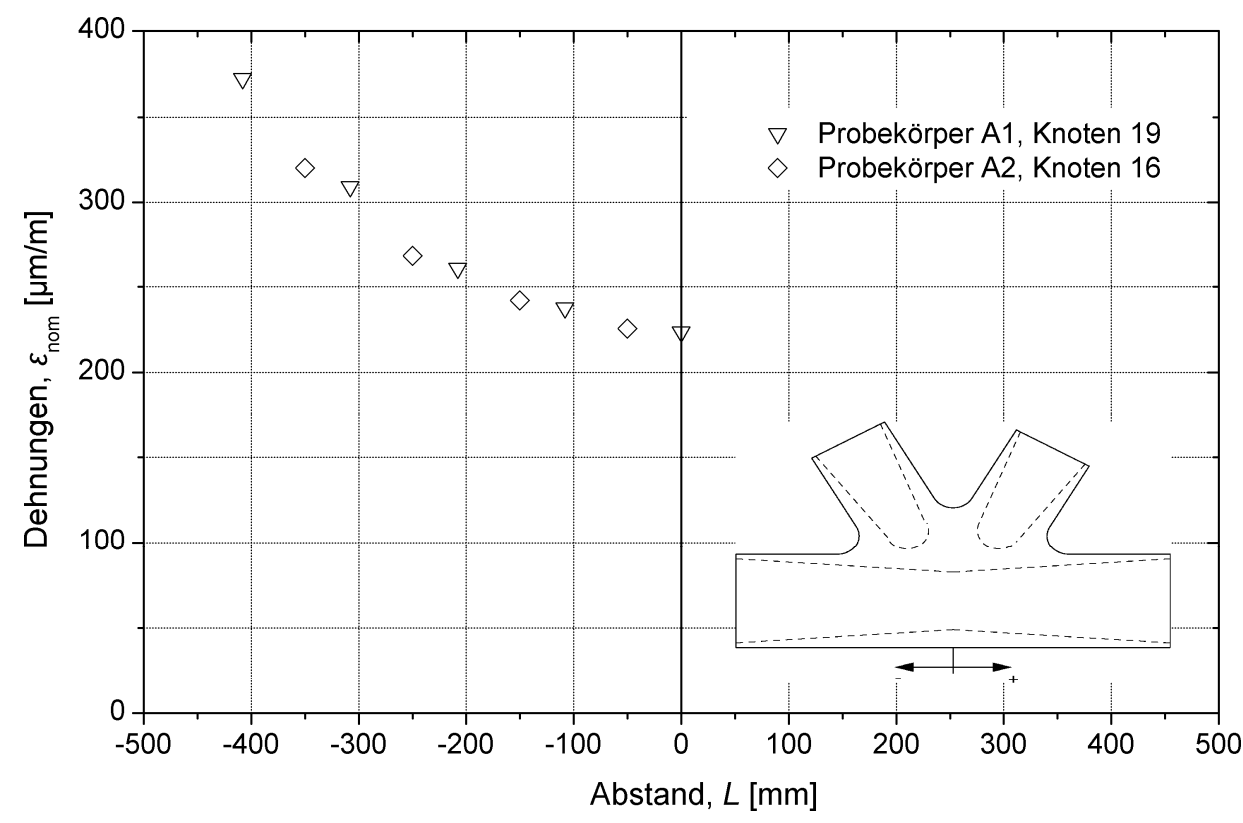

Fig. 3.15: Dehnungen auf Unterseite des Untergurtstutzens, Serie A

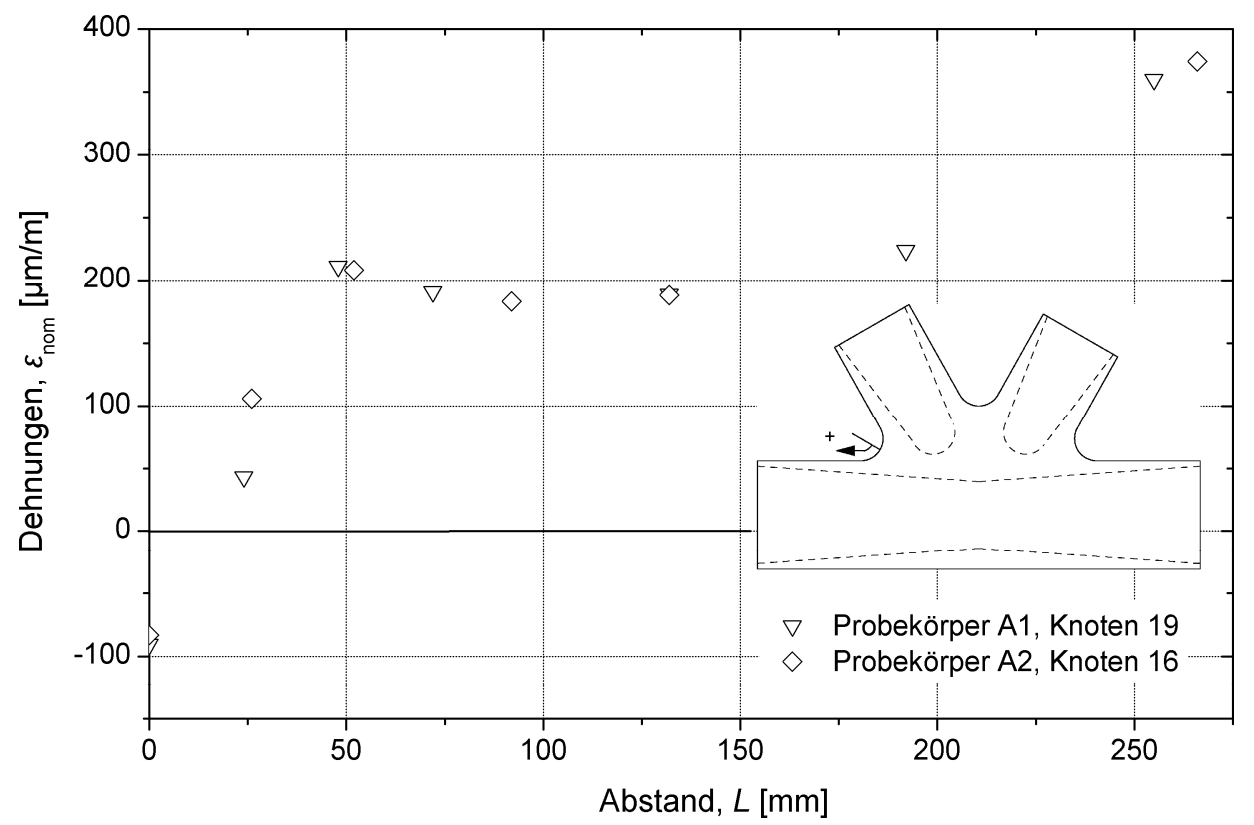

Fig. 3.16: Dehnungen auf der Oberseite des Untergurtstutzens, Serie A

\subsubsection{Ermüdungsversuche}

\subsubsection{Generelle Beurteilung}

Die Versuchsergebnisse haben sehr eindeutig gezeigt, dass für die gewählten Parameter und die in den Versuchen vorliegende Konfiguration die Schweissnähte massgebend sind für die Ermüdungs- 
festigkeit. In den Gusskomponenten wurden keinerlei Ermüdungsrisse beobachtet. Mit dem Nachweis von vorhandenen Ungänzen (Abschnitt 3.5.3) wird im Anschluss an die Ermüdungsversuche gezeigt, dass die Gusskomponenten von sehr guter Qualität waren und keine wesentlichen Ungänzen aufwiesen. Die Schweissnahtreparaturen weisen eine relative niedrige Ermüdungsfestigkeit auf.

\subsubsection{Rissfortschrittsdaten}

Alle in den Probekörpern beobachteten Ermüdungsrisse traten in den Stumpfschweissnähten zwischen den Untergurtstutzen und dem anschliessenden Hohlprofil auf. Diese Schweissnähte waren im Vergleich zu den Schweissnähten bei den Diagonalstutzen höher belastet. Zuerst traten die Risse an den höchstbeanspruchten Stellen 1 und 2 auf (Fig. 3.17). Im Fall des Probekörpers A1 wurden diese beiden Rissstellen repariert und der Ermüdungsversuch weitergeführt. Der nächste Ermüdungsriss trat dann an der Stelle 3 auf. Während der verschiedenen Ermüdungsversuche traten auch Risse in den Schweissnähten der Zugdiagonalen, die zum Versuchsrahmen gehören, auf (Fig. 3.1). Diese Ergebnisse werden ebenfalls in die Versuchsresultate aufgenommen.

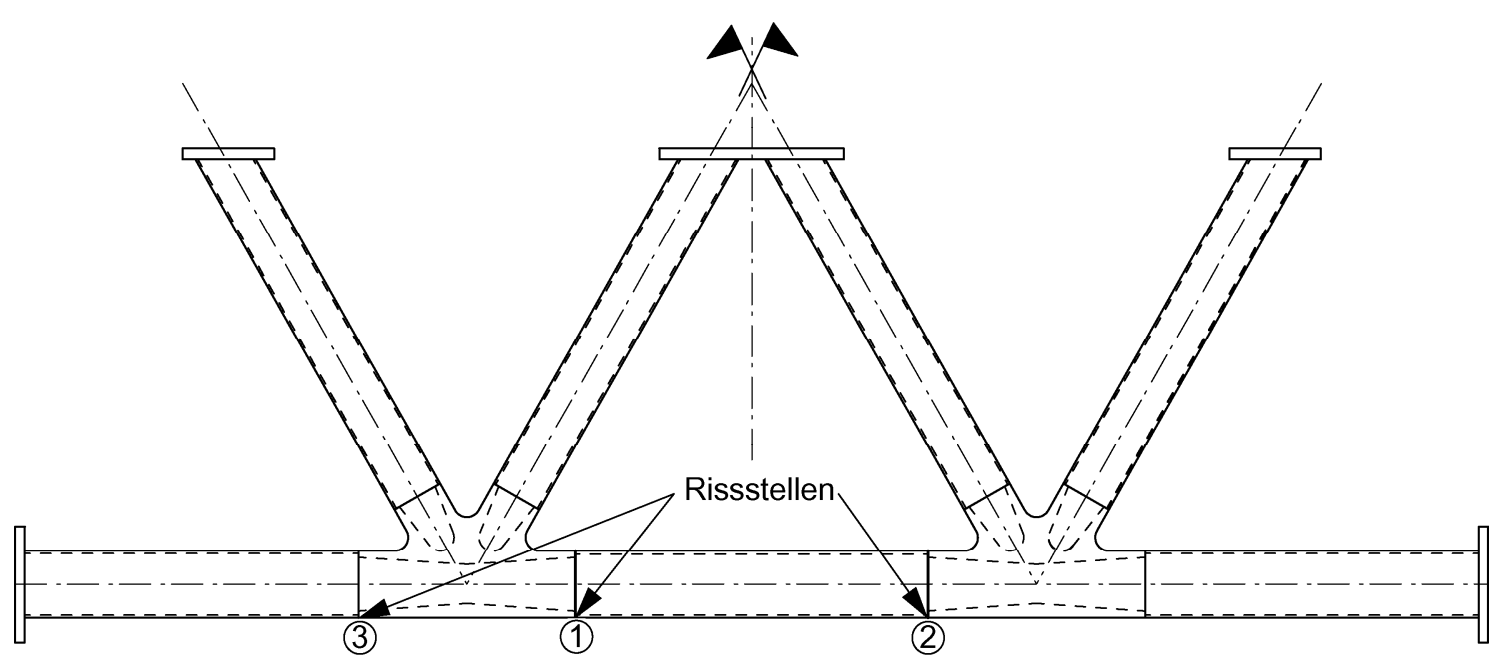

Fig. 3.17: Rissstellen im Probekörper A1
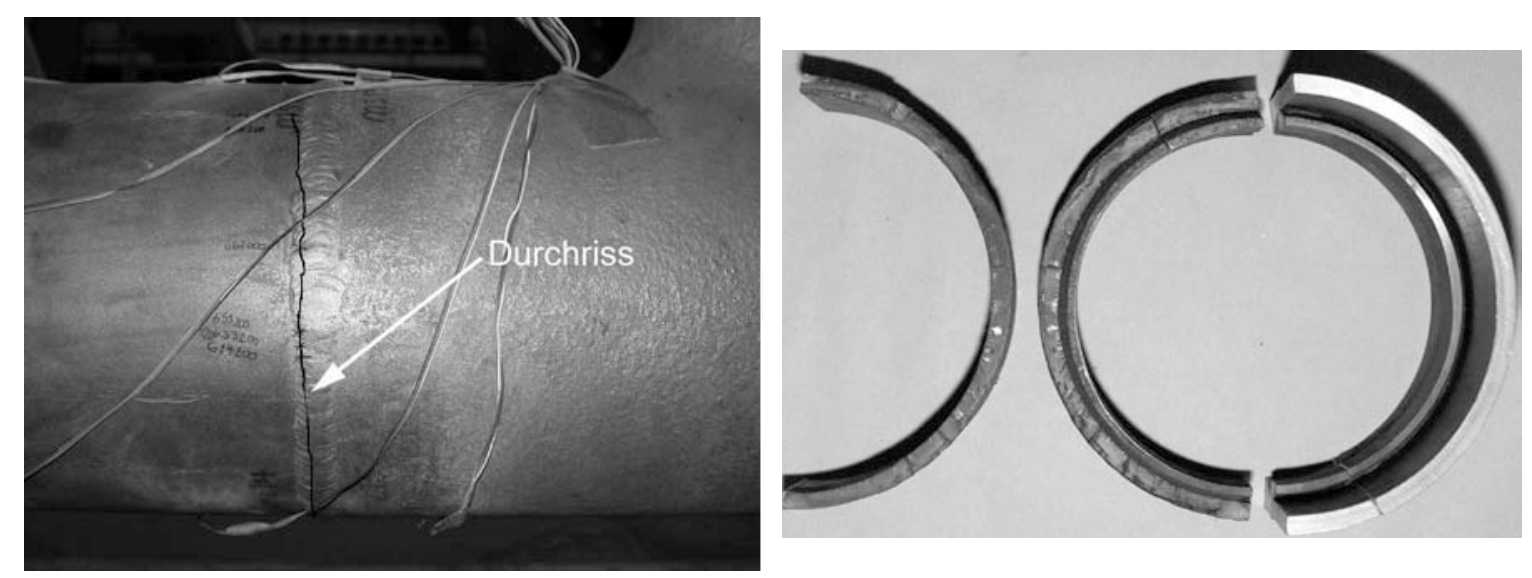

a) Stumpfnaht, Knoten 3, Probekörper B0

b) Rissbruchfläche, Knoten 3, Probekörper BO

Fig. 3.18: Beispiel eines Durchrisses in einer Stumpfnaht 
Die Rissinitiierung fand stets an der Wurzelseite in Schweissnahtmitte statt, die Risse breiteten sich also von der Hohlprofilinnenseite her aus. Sie konnten daher erst beim Wanddurchbruch, der auf der Unterseite des Untergurts auftrat, visuell beobachtet werden. Nach dem Durchbruch breiteten sich die Risse radial entlang der Schweissnahtmitte aus, im Lauf des Risswachstums dann auch entlang des Schweissnahtfusses oder entlang der Wärmeeinflusszone auf der Hohlprofilseite (Beispiel Fig. 3.18 a)).

Fig. 3.18 a) zeigt als Beispiel den Durchriss in der Schweissnaht im Untergurt bei Knoten 3 (Probekörper B0). Eine Rissfläche des Durchrisses ist in Fig. 3.18 b) abgebildet. Auf dem Bild ist auch die für die Serie B verwendete Schweissbadsicherung zu erkennen. Anhand der Fächer auf der Rissfläche ist zu erkennen, dass an mehreren Stellen entlang der Schweissnahtwurzel Risse initiierten und sich ausbreiteten.

Fig. 3.19 und Fig. 3.20 zeigen als Beispiel das Risswachstum in den in der Mitte des Untergurts liegenden Schweissnähten. Beim Probekörper A1 wurde zuerst der Riss beim Knoten 15 und anschliessend beim Knoten 19 (Stelle 1 und 2 in Fig. 3.17) entdeckt. Nach der Reparatur der beiden Risse (dies ist der Grund, warum der Riss bei Knoten 19 nur $100 \mathrm{~mm}$ lang ist) wurde der Versuch weitergeführt, bis ein Riss an der Stelle 3 auftrat. Dieser Riss ist in Fig. 3.19 nicht abgebildet.

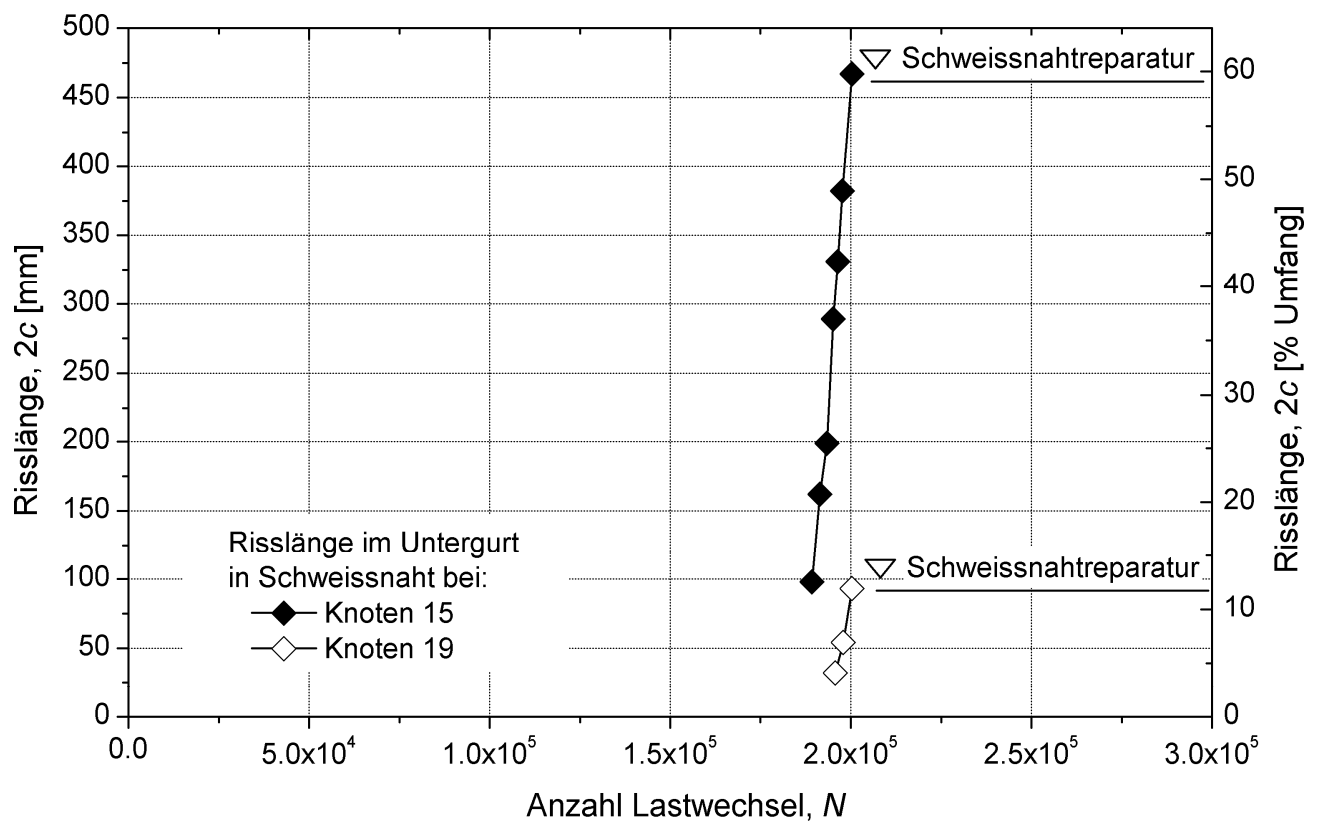

Fig. 3.19: Risswachstum Probekörper A1, Mitte Untergurt

Die Risslängen, bei denen die Risse zum ersten Mal visuell als Durchrisse entdeckt wurden, betragen zwischen 25 und $150 \mathrm{~mm}$. Es ist zu beobachten, dass die Risswachstumsgeschwindigkeit für den Durchriss sehr steil ansteigt. Daher konnte der exakte Zeitpunkt des Wanddurchbruchs während des Versuchs nur schwer beobachtet werden, was den oben erwähnten grossen Risslängenbereich bei der Entdeckung der Durchrisse erklärt. Die Anzahl Lastwechsel ab dem Zeitpunkt der Rissentdeckung bis zum Abbruch des Versuchs bei einer Risslänge von ungefähr 50 \% des Hohlprofilumfangs ist im Verhältnis zur gesamten Lebensdauer sehr gering und beträgt höchstens $10 \%$. 
Im Gegensatz zu den beiden in Fig. 3.19 dargestellten Rissen liegen in Fig. 3.20 die Zeitpunkte des Wanddurchbruchs der beiden beobachteten Durchrisse weit auseinander: Die Anzahl Lastwechsel bei der Rissentdeckung beträgt ca. das Doppelte. Der Durchriss bei Knoten 3 (Stelle 2 in Fig. 3.17) wurde repariert und anschliessend der Versuch weitergeführt bis der Durchriss bei Knoten 8 (Stelle 1 in Fig. 3.17) auftrat. Die unterschiedlichen Rissfortschrittsgeschwindigkeiten lassen auf einen weit kleineren Schweissnahtfehler als Auslöser des Ermüdungsrisses bei Knoten 8 schliessen.

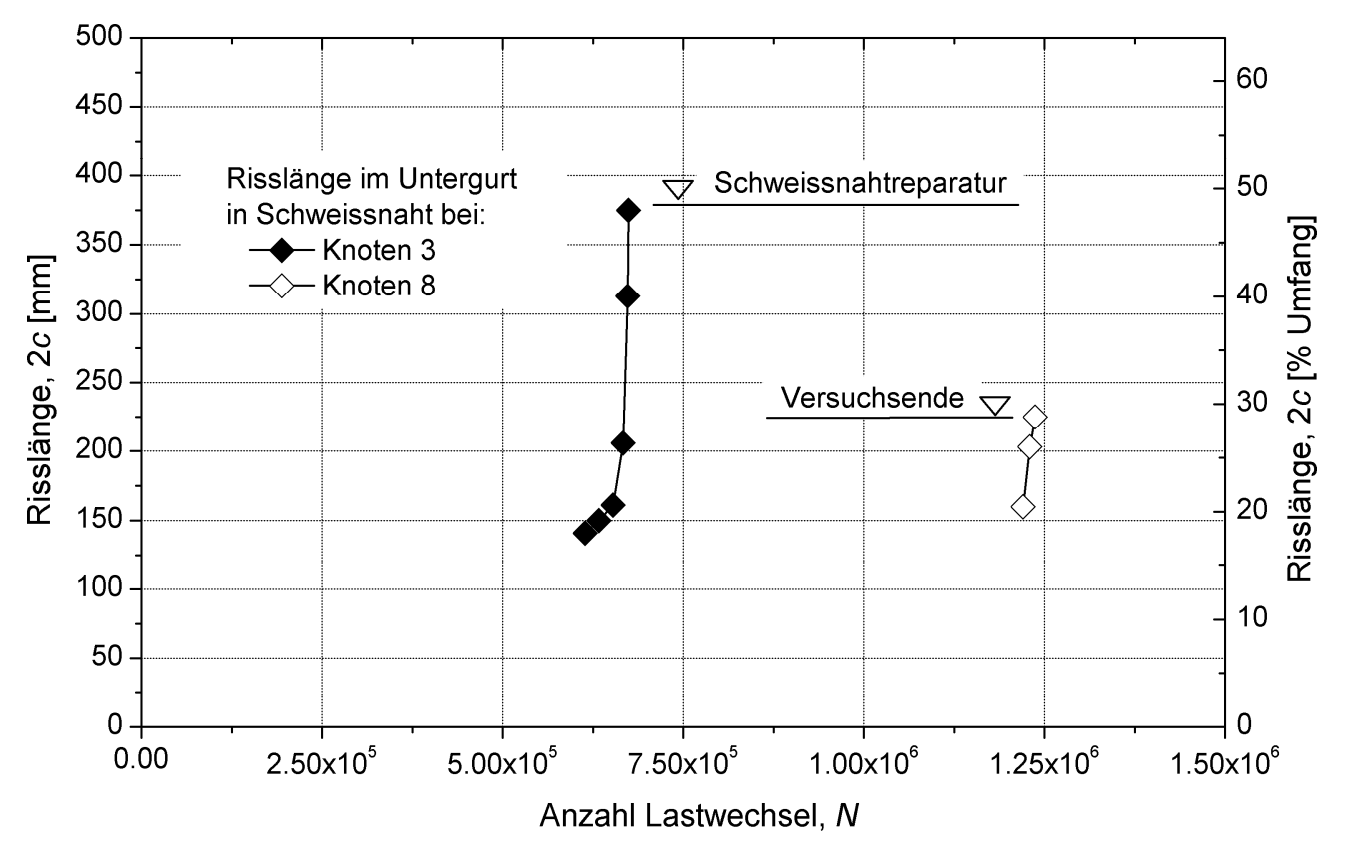

Fig. 3.20: Risswachstum Probekörper B0, Mitte Untergurt

\subsubsection{S-N Versuchsresultate}

Fig. 3.21 präsentiert die gesamten Ergebnisse aus den Ermüdungsversuchen der beiden Versuchsserien A und B sowie der Voruntersuchung in einem doppellogarithmischen Diagramm. Die tabellarische Auflistung der Versuchsdaten befindet sich im Anhang B. Die in der Schweissnaht auftretende Spannungsschwingbreite $\Delta \sigma_{\text {SCF }}$ wird aus den extrapolierten Dehnungsmesswerten (Tab. 3.8) und dem Lastverhältnis von $Q_{\min } / Q_{\max }=0.1$ abgeleitet. Dabei handelt es sich um eine Differenz aus Nominalspannungen. Um die Spannungskonzentrationen infolge der exzentrischen Anschlüsse mit zu berücksichtigen, werden die Spannungskonzentrationsfaktoren (stress concentration factor, SCF) in den Stumpfschweissnähten nach [DNV 2001] berechnet (Abschnitt 2.4.3). Die Werte sind in Tab. 3.9 zusammengefasst.

\begin{tabular}{lccccccc}
\hline & \multicolumn{3}{c}{ Probekörper A1 \& A2 } & \multicolumn{2}{c}{ Probekörper B0 } & \multicolumn{2}{c}{ Probekörper B1 \& B2 } \\
\hline Schweissdetail & $\begin{array}{c}\text { Untergurt } \\
\text { (Fig. 3.5) }\end{array}$ & Diagonale & $\begin{array}{c}\text { Diagonale Ver- } \\
\text { suchsrahmen }\end{array}$ & $\begin{array}{c}\text { Untergurt } \\
\text { (Fig. 3.7) }\end{array}$ & Diagonale & Untergurt & Diagonale \\
\hline SCF [-] & 1.17 & 1.26 & 1.02 & 1.16 & 1.18 & 1.10 & 1.12 \\
\hline
\end{tabular}

Tab. 3.9: Spannungskonzentrationsfaktoren in den Schweissnahtdetails 


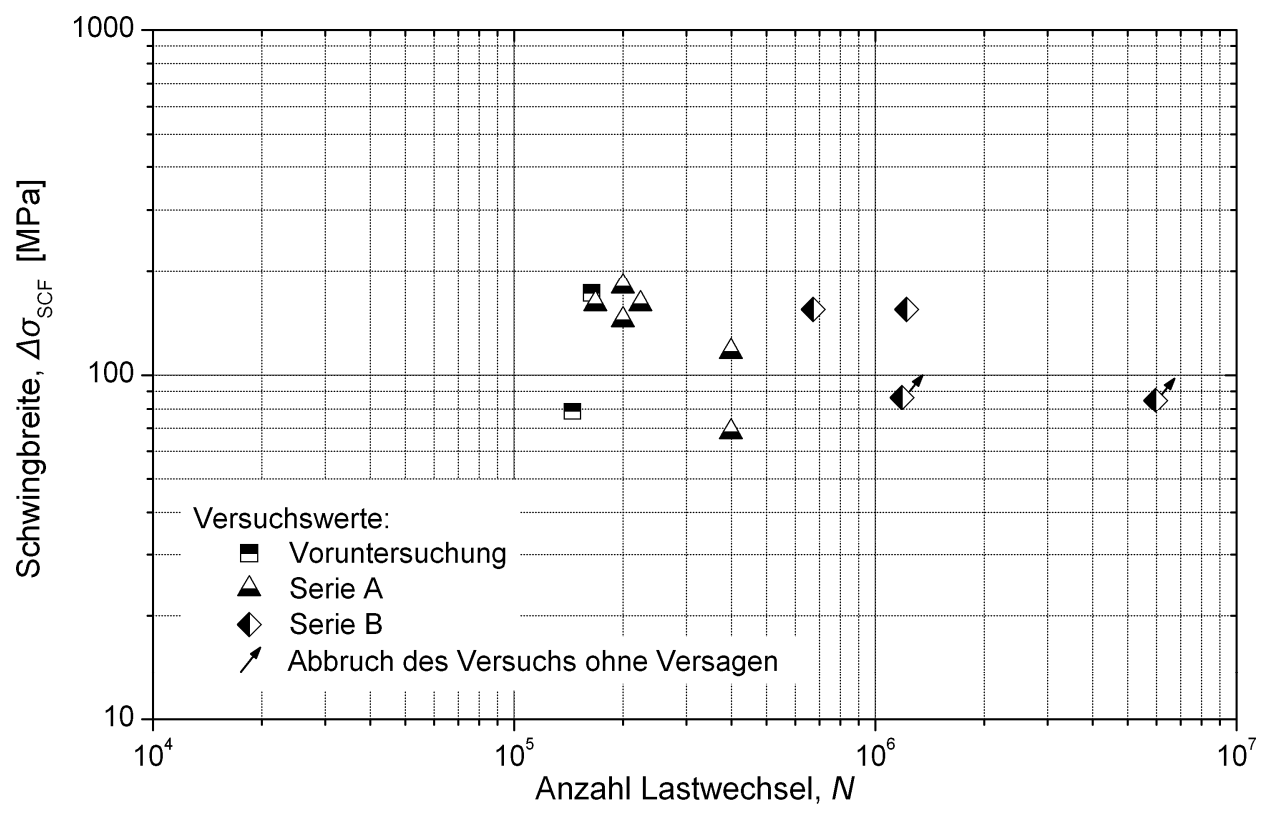

Fig. 3.21: Versuchsresultate, Übersicht

Aus den Versuchsergebnissen kann nun eine Ermüdungsfestigkeitskurve ermittelt werden. Sie ist eine Parallele zur Regressionsgeraden der Messwerte mit einer auf $m=3$ festgelegten Steigung und einem horizontalen Abstand, der zweimal der Standardabweichung $s_{\mathrm{N}}$ entspricht.

Da für die statistische Auswertung der Messwerte nur eine kleine Stichprobe pro Versuchsserie vorhanden ist, widerspiegelt die Standardabweichung der Messwerte nicht die zu erwartende Standardabweichung einer grösseren Stichprobe. Daher wird für die Standardabweichung $s_{\mathrm{N}}$ ein Wert aus der Literatur übernommen. In [ECCS-CECM-EKS 1987] wird für geschweisste Träger ein Schätzwert zwischen 0.15 und 0.21 angegeben. Die Standardabweichung $s_{\mathrm{N}}$ hängt von der Streuung der Grösse, Form und Dichte der Kerben, von der örtlichen Spannungskonzentration, der Grösse des Konstruktionsdetails, der Spannungsrichtung, den metallurgischen Einflüssen, den Eigenspannungen, der Ermüdungsrissform und dem Schweissvorgang ab. Für eine konservative Auswertung wird hier der Wert 0.20 gewählt, woraus ein horizontaler Abstand zwischen der Ermüdungsfestigkeitskurve und der Regressionsgeraden von $2 \cdot s_{\mathrm{N}}=0.4$ resultiert.

Zur Auswertung werden die Versuchsergebnisse in verschiedenen Gruppen betrachtet. Da die Schweissdetails aus der Voruntersuchung und der Versuchsserie A die gleichen Abmessungen $(t=10 \mathrm{~mm})$ haben und ohne Badsicherung ausgebildet sind, werden diese zusammen in Fig. 3.22 betrachtet. Für die Stumpfnähte ohne Schweissbadsicherung ergeben die Versuchsergebnisse eine Ermüdungsfestigkeitskurve mit $\Delta \sigma_{\mathrm{C}}=54 \mathrm{MPa}$. Zur Orientierung und als Vergleich werden Ermüdungsfestigkeitskurven für Stumpfnähte ohne Schweissbadsicherung nach den in Abschnitt 2.4.2 zitierten Normen abgebildet. Die Ermüdungsfestigkeitskurve mit $\Delta \sigma_{\mathrm{C}}=54 \mathrm{MPa}$ entspricht in etwa den Kerbfällen F3 und F2 nach [DNV 2001] und [BS 7608:1993]. Der Kerbfall 90 nach [prEN 19931-9:2003] liegt hingegen weit darüber.

Die Ergebnisse der fehlerbehafteten Stumpfnähte, die in der Versuchsserie A und in den Voruntersuchungen vorkamen, werden gesondert in Fig. 3.23 angegeben. Die fehlerbehafteten Nähte befanden sich in den Zugdiagonalen des Versuchsrahmens. Obwohl für die Schweissnähte die Bewertungsgruppe B nach [SIA 263] verlangt und mit Ultraschalluntersuchungen überprüft wurde, er- 
füllte die Qualität der Wurzellage bei diesen Schweissnähten die Anforderungen nicht. Die fehlerbehafteten Nähte wiesen Bindefehler von $6 \mathrm{~mm}$ (Serie A) und $8 \mathrm{~mm}$ (Voruntersuchung) auf. Die Fehler wurden erst bei der Bruchflächenauswertung festgestellt.

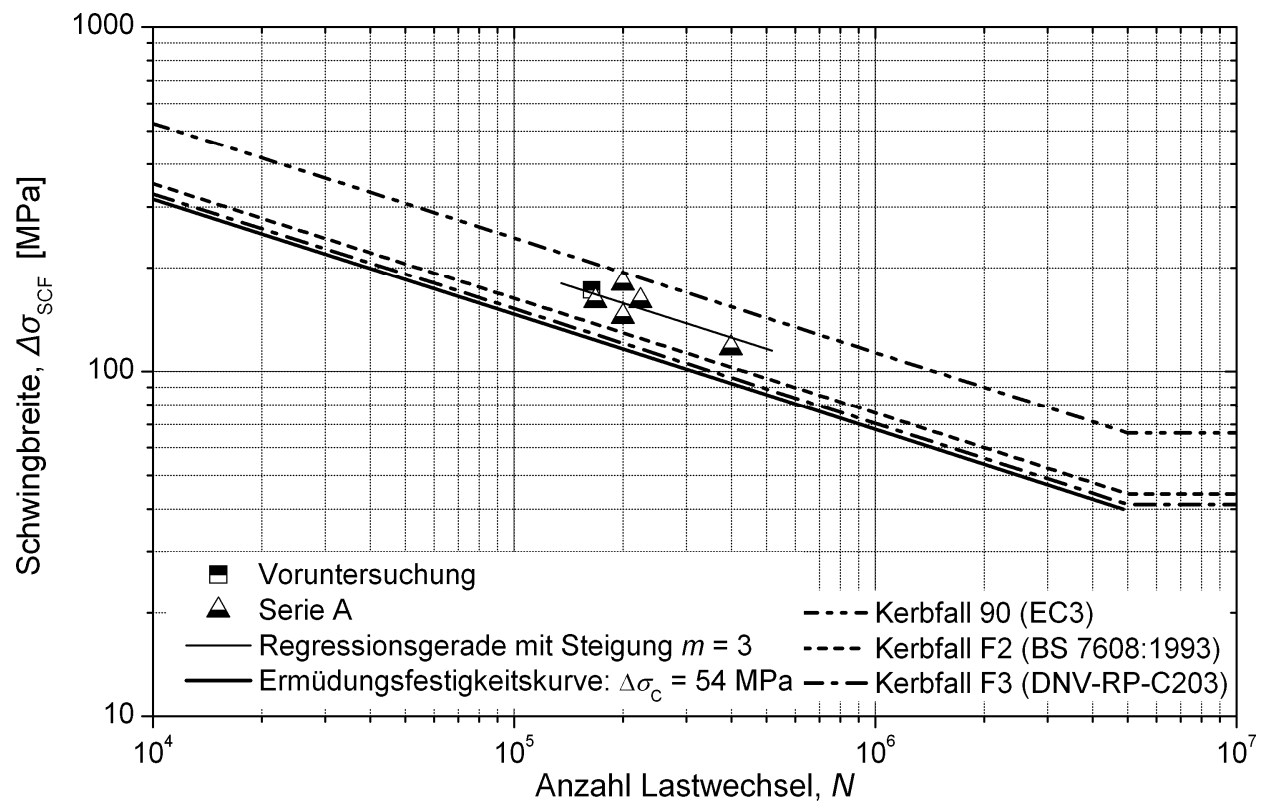

Fig. 3.22: Versuchsresultate aus der Voruntersuchung und der Serie A, ohne Badsicherung

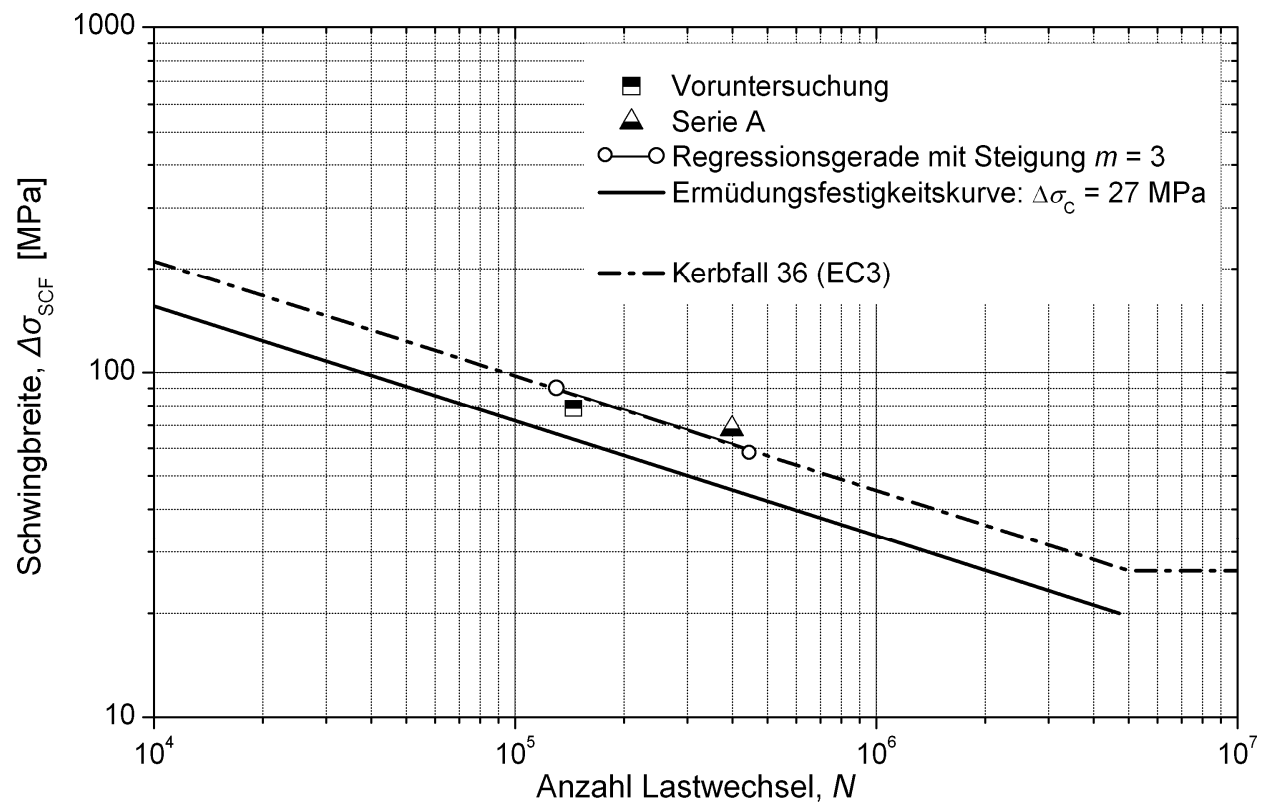

Fig. 3.23: Versuchsresultate aus der Voruntersuchung und der Serie A, ohne Badsicherung, fehlerbehaftete Nähte

Für die fehlerbehafteten Nähte ohne Schweissbadsicherung ergibt sich eine Ermüdungsfestigkeitskurve mit $\Delta \sigma_{\mathrm{C}}=27 \mathrm{MPa}$. Diese liegt unter dem niedrigsten Kerbfall 36 nach EC3 [prEN 1993-19:2003], der für einseitig geschweisste Stumpfstösse zwischen ebenen Blechen, bei denen mit zerstörungsfreien Prüfungen eine nicht durchgeschweisste Naht nachgewiesen wird, gilt. Da es sich hier 
jedoch um eine sehr kleine Stichprobe von Versuchsergebnissen handelt, hat diese Auswertung nur eine beschränkte Aussagekraft.

In Fig. 3.24 sind die Versuchsergebnisse der Serie B abgebildet. Obwohl bei dieser Serie die Wanddicken der Probekörper variieren (B0: $t=10 \mathrm{~mm}$, B1 und B2: $t=17.5 \mathrm{~mm}$ ), sind die Nähte mit einer Badsicherung ähnlich ausgebildet. Die Badsicherung führte durch eine gute Aufschweissung zu einer weit besseren Qualität der Wurzellage. Der Ermüdungsversuch für den Probekörper B2 wurde bei $6 \cdot 10^{6}$ Lastwechseln eingestellt, ohne dass ein Ermüdungsbruch beobachtet werden konnte. Der zuletzt durchgeführte Versuch des Probekörpers B1 musste auf Grund sehr vieler aufgetretener Schäden im Versuchsrahmen bereits nach 1.2 $10^{6}$ Lastwechseln abgebrochen werden. Fig. 3.24 zeigt daher für die Stumpfnähte in den Untergurten dieser beiden Probekörper nur die Ergebnisse bei Abbruch des Versuchs ohne Versagen. Für die Stumpfnähte mit Schweissbadsicherung ergibt sich eine Ermüdungsfestigkeitskurve mit $\Delta \sigma_{\mathrm{C}}=87 \mathrm{MPa}$. Zur Orientierung und als Vergleich werden Ermüdungsfestigkeitskurven für Stumpfnähte mit Schweissbadsicherung nach den in Abschnitt 2.4.2 zitierten Normen abgebildet. Die Ermüdungsfestigkeitskurve mit $\Delta \sigma_{\mathrm{C}}=87 \mathrm{MPa}$ liegt etwas über den Kerbfällen F, die in [DNV 2001] und [BS 7608:1993] angegeben sind. Auch hier wird auf die beschränkte Aussagekraft der Auswertung auf Grund der sehr wenigen Versuchsergebnisse hingewiesen.

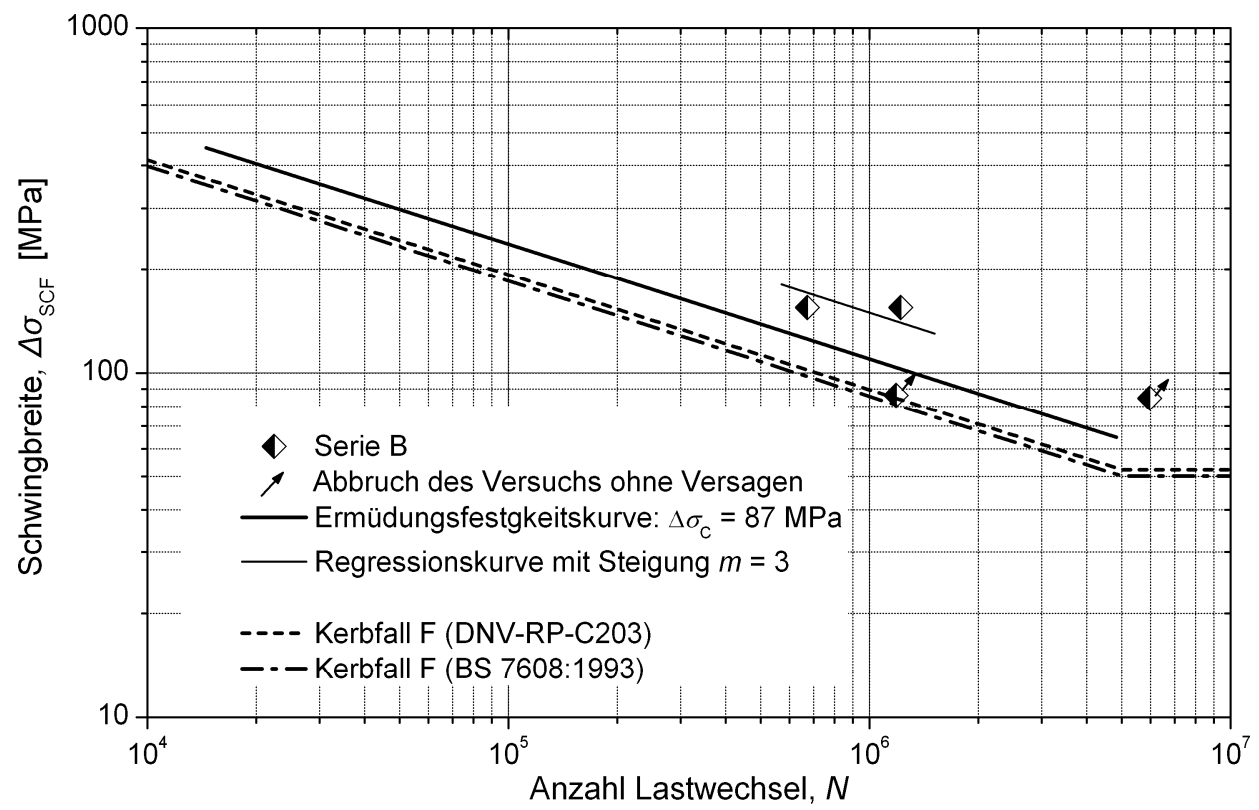

Fig. 3.24: Versuchsresultate der Serie B, mit Badsicherung

Die Auswertung der Versuchsergebnisse zeigt, dass Stumpfnähte mit Badsicherung $\left(\Delta \sigma_{\mathrm{C}}=87 \mathrm{MPa}\right)$ gegenüber denjenigen ohne Badsicherung $\left(\Delta \sigma_{\mathrm{C}}=54 \mathrm{MPa}\right)$ eine höhere Ermüdungsfestigkeit haben. Daraus wird geschlossen, dass die durch die Badsicherung eingeführte Kerbwirkung an der Wurzelseite weniger aggressiv ist als diejenige von Bindefehlern bei Stumpfnähten ohne Badsicherung. Diese Tendenz wird auch durch die den Normen [DNV 2001] [BS 7608:1993] entsprechenden Kerbfälle bestätigt.

In Fig. 3.25 werden für die Reparaturen der Schweissnähte die Ergebnisse aus den Ermüdungsversuchen zusammengestellt. Allerdings können nur beschränkte Aussagen bezüglich der Ermüdungs- 
festigkeit der reparierten Schweissnähte gemacht werden. Als Vergleich und Orientierung wird die Ermüdungsfestigkeitskurve für den Kerbfall 50 nach EC3 [prEN 1993-1-9:2003] für das Konstruktionsdetail mit Gurtlamellen auf Walzprofilen angegeben.

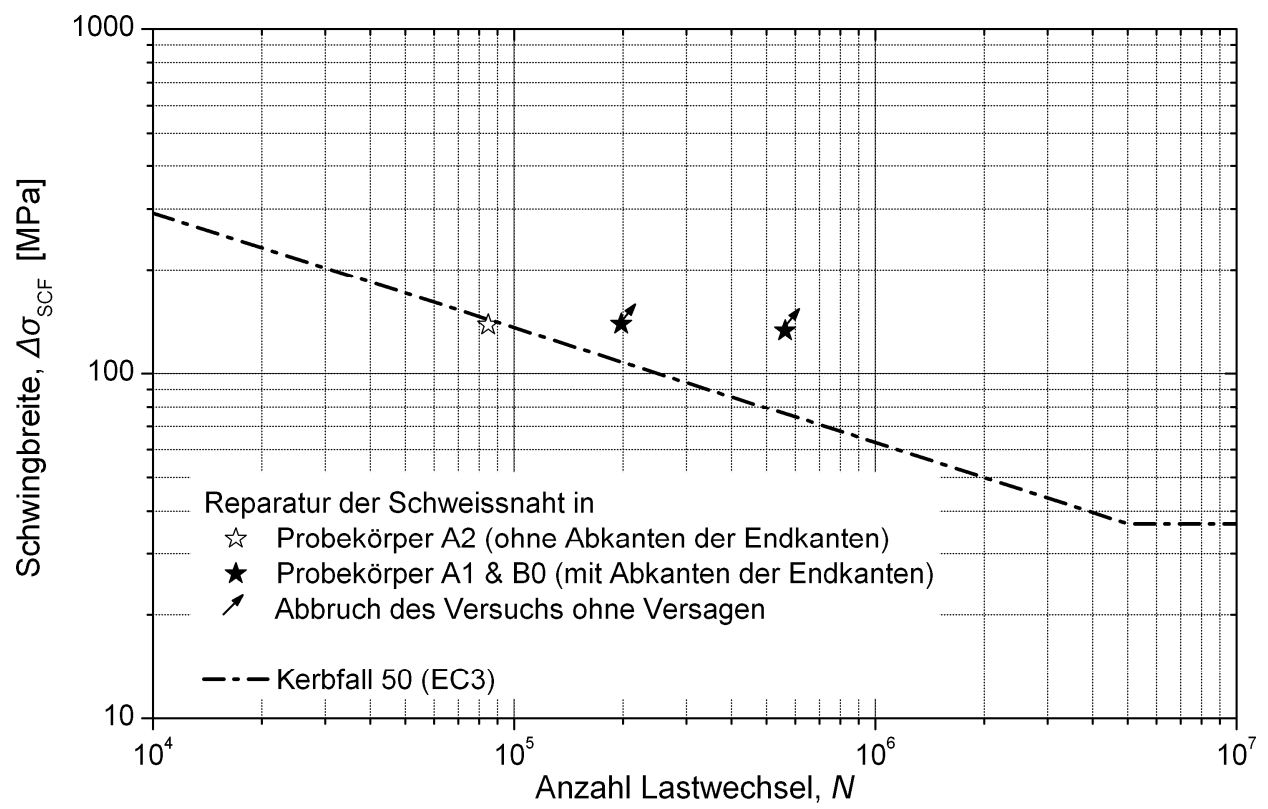

Fig. 3.25: Resultate zu den reparierten Schweissnähten

Der Probekörper A2 wurde zunächst an der Stelle 1 (Fig. 3.17) mit Halbschalen, deren Endkanten nicht nach innen abgekantet waren, repariert. Nach dem Auftreten eines Ermüdungsrisses an der Stelle 2 wurde diese ebenfalls repariert. Da sich die Reparatur ohne Abkanten der Endkanten nicht als sehr ermüdungsfest erwies, konnte der Versuch nach der zweiten Reparatur nicht lange weitergefahren werden bis es zum Bruch der ersten Reparaturstelle kam. Der Ermüdungsriss ging von der Schweissnahtwurzel einer Endkante aus und breitete sich sehr rasch radial aus.

Für die Reparaturen der Schweissnähte im Untergurt der Probekörper A1 und B0 wurden die Endkanten der Halbschalen abgekantet. Für diese beiden Probekörper wurden die Versuche abgebrochen, bevor es zum Bruch der Reparaturen kam. Aus diesem Grund sind die Ergebnisse in Fig. 3.25 bei Abbruch des Versuchs ohne Versagen gekennzeichnet. Durch das Abkanten der Endkanten scheint die Ermüdungsfestigkeit der Reparaturstellen höher zu liegen.

\subsubsection{Vergleich mit externen Versuchsresultaten}

Im Rahmen des Baus des Lehrter Bahnhofs in Berlin wurden unter der Leitung der Versuchanstalt für Stahl, Holz und Steine der Universität Karlsruhe Versuche zum Tragverhalten von geschweissten Verbindungen aus Stahlguss (GS-20 Mn 5 V) und Stahl (S355 J2) durchgeführt [Mang et al. 2001]. Es wurden Ermüdungsversuche an Kleinproben und Grossbauteilen unter einem Lastverhältnis $Q_{\min } / Q_{\max }=0.1$ durchgeführt. Die Kleinproben bestanden aus ebenen, geschweissten, $25 \mathrm{~mm}$ bzw. $40 \mathrm{~mm}$ dicken Platten, die teils mit, teils ohne Badsicherung ausgebildet waren (Fig. 3.26). Die Grossbauteilversuche wurden an geschweissten Hohlprofilen durchgeführt, deren Durchmesser $267 \mathrm{~mm}$ und deren Wanddicke $20 \mathrm{~mm}$ betrugen. Die Grossbauteile wurden ebenfalls teils mit und teils ohne Badsicherung ausgebildet. In Fig. 3.27 ist das Schweissdetail mit Badsicherung, die als angegossene Nase am Gussstück ausgebildet ist, dargestellt. 
In Fig. 3.26 werden die Ergebnisse der Ermüdungsversuche an Kleinproben aus [Mang et al. 2001] mit der jeweiligen Regressionsgerade (hier mit variabler Steigung) sowie die Versuchsresultate der vorliegenden Arbeit in doppellogarithmischer Auftragung dargestellt.

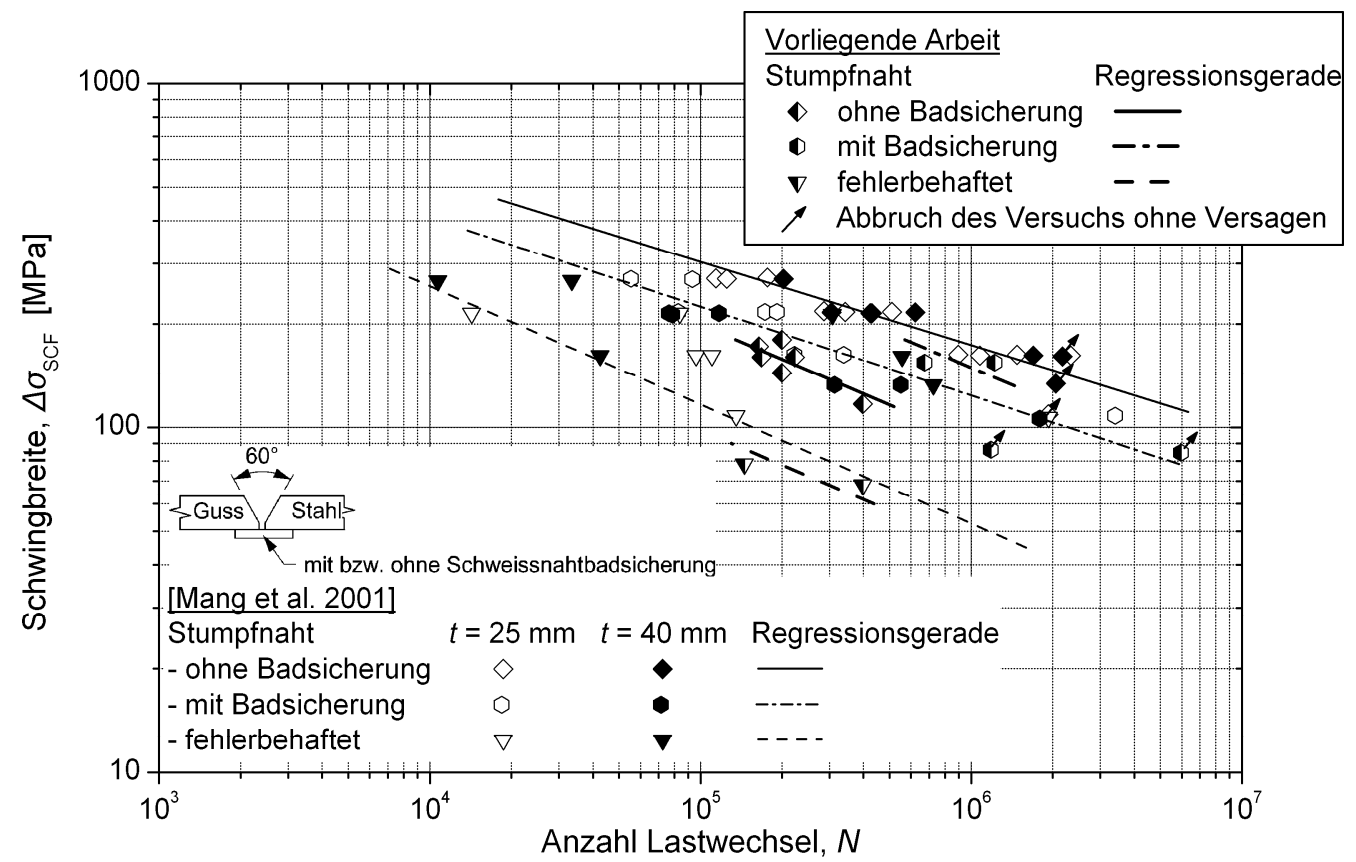

Fig. 3.26: Versuchsresultate der vorliegenden Arbeit im Vergleich zu Kleinproben aus [Mang et al. 2001]

Es zeigt sich, dass bei den Kleinproben - im Gegensatz zu den Versuchsresultaten der vorliegenden Arbeit - die Ergebnisse für die Stumpfnähte ohne Badsicherung etwas höher liegen als diejenigen für die Schweissnähte mit Badsicherung. Wie aus dem Diagramm deutlich wird, liegen die vorliegenden Versuchsresultate „ohne Badsicherung“ sehr deutlich unter den Ergebnissen aus [Mang et al. 2001]. Dies könnte einerseits daran liegen, dass die Schweissnahtqualität der Kleinproben wesentlich besser ist als diejenige der vorliegenden Probekörper. Die Ermüdungsfestigkeit von Stumpfschweissnähten ohne Badsicherung hängt sehr stark von der Qualität der Schweissnahtwurzel und damit von den handwerklichen Fertigkeiten des Schweissers ab. Die Streuung der Versuchsresultate kann entsprechend gross sein. Aus diesem Grund verlangt die Deutschen Bahn AG grundsätzlich die Ausführung der Stumpfnähte mit Badsicherung [Deutsche Bundesbahn 1993]. Die grosse Diskrepanz der Ergebnisse könnte auch mit der unterschiedlichen Verteilung der Schweisseigenspannungen erklärt werden. Da die Kleinproben aus ebenen, geschweissten, $25 \mathrm{~mm}$ bzw. $40 \mathrm{~mm}$ dicken Platten bestehen, können die Schweisseigenspannungen in Wanddickenrichtung eine andere Verteilung haben als bei einem Stumpfstoss zwischen zwei Rundhohlprofilen. Es ist möglich, dass bei einem Lastverhältnis $Q_{\min } / Q_{\max }=0.1$ ein Riss an der Schweissnahtwurzel infolge von Druckeigenspannungen während eines Teils der Spannungsamplitude keine Zugspannung erfährt. In diesem Fall ist der Spannungsintensitätsfaktor $K_{\mathrm{op}}(a)$, bei dem die Risslippen tatsächlich geöffnet werden, grösser als $K_{\min }(a)$ und die effektive Differenz des Spannungsintensitätsfaktors $\Delta K_{\text {eff }}(a)$, die zum Risswachstum führt, nur $K_{\max }(a)-K_{\text {op }}(a)$. Dieser kleinere Wert von $\Delta K_{\text {eff }}(a)$ könnte ein Grund für den Unterschied zwischen den vorliegenden Versuchsresultaten und den Ergebnissen aus [Mang et al. 2001] sein. Druckspannungen im Wurzelbereich der Kleinproben 
könnten auch infolge von Sekundärbiegemomenten aus Exzentrizitäten in der Schweissnaht entstehen.

Die vorliegenden Resultate „mit Badsicherung“ fallen im Vergleich zu den Ergebnissen in [Mang et al. 2001] etwas besser aus. Die fehlerbehafteten Nähte ergeben in beiden Fällen sehr schlechte Ergebnisse.

Die in [Mang et al. 2001] aufgeführten Ergebnisse der Grossbauteilversuche beruhen auf Nominalspannungen in den Stahlhohlprofilen. Um die Ergebnisse mit den vorliegenden Untersuchungen vergleichen zu können, wird die durch den aussermittigen Anschluss hervorgerufenen Spannungskonzentrationen in der Stumpfnaht mitberücksichtigt. Dazu wird der Spannungskonzentrationsfaktor (SCF) nach [DNV 2001] (Abschnitt 2.4.3) berechnet; er beträgt für das in Fig. 3.27 dargestellte Schweissnahtdetail mit Badsicherung 1.29. Die mit dem SCF korrigierten Ergebnisse aus [Mang et al. 2001] werden in Fig. 3.27 dargestellt. Die Auswertung der Versuchsergebnisse aus [Mang et al. 2001] entsprechend der zu Beginn des Abschnitts erklärten Vorgehensweise zeigt, dass in der vorliegenden Untersuchung die Schweissnähte mit Badsicherung eine höhere Ermüdungsfestigkeit aufweisen. Allerdings muss berücksichtigt werden, dass nur zwei Versuchergebnisse vorliegen und daher keine abschliessende Aussage gemacht werden kann. Zur Orientierung und als Vergleich werden Ermüdungsfestigkeitskurven für Stumpfnähte mit Schweissbadsicherung nach den in Abschnitt 2.4.2 zitierten Normen abgebildet. Die Ermüdungsfestigkeitskurve mit $\Delta \sigma_{\mathrm{C}}=63$ $\mathrm{MPa}$ für [Mang et al. 2001] liegt etwas unter den Kerbfällen F, die in [DNV 2001] und [BS 7608:1993] angegeben sind.

Die Versuchsresultate ohne Badsicherung liegen leicht über den Ergebnissen aus [Mang et al. 2001]. Da es sich dort jedoch um Ergebnisse bei Abbruch des Versuchs ohne Versagen handelt, ist ein direkter Vergleich nicht möglich.

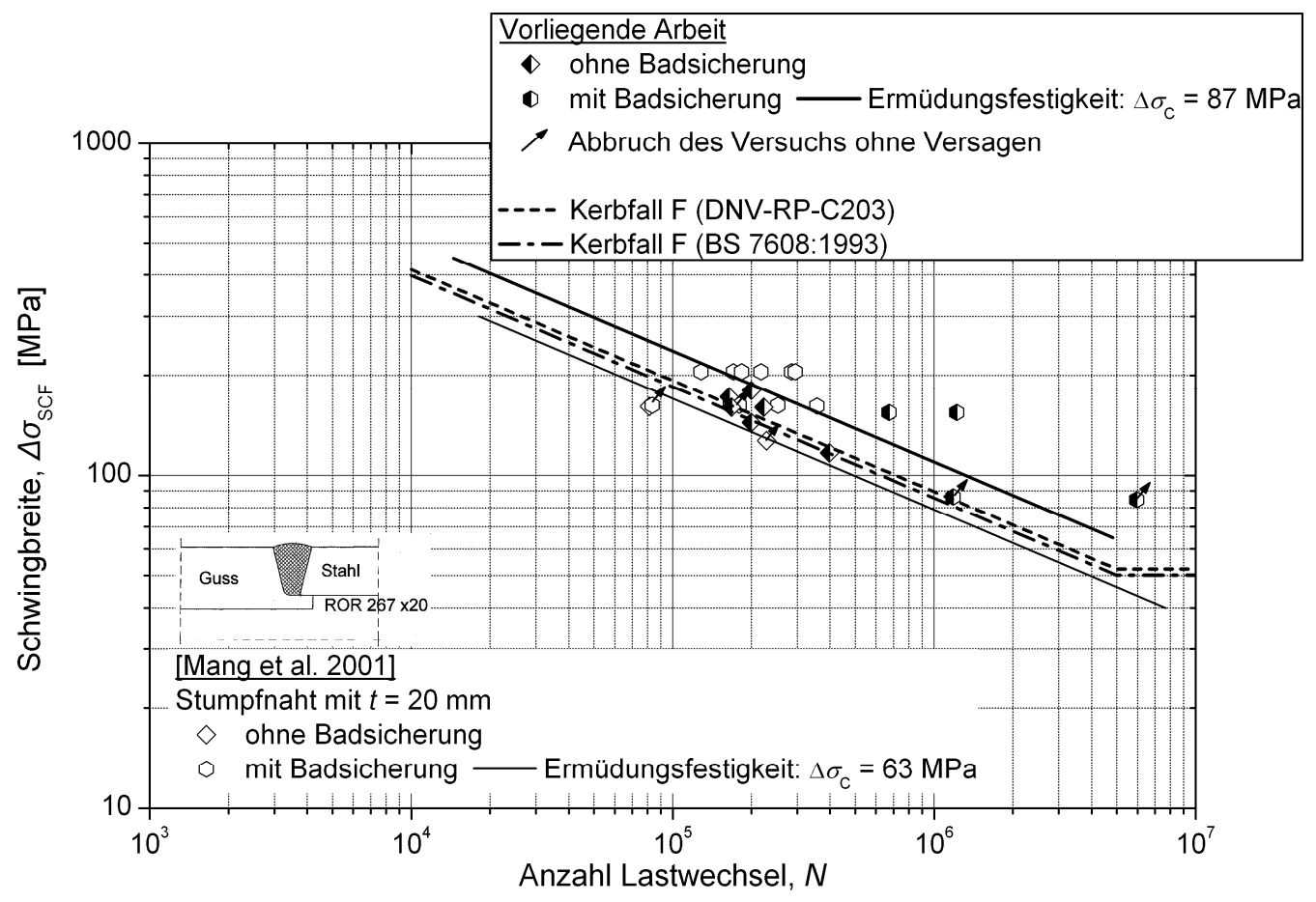

Fig. 3.27: Versuchsresultate der vorliegenden Arbeit im Vergleich zu den Grossbauteilversuchen aus [Mang et al. 2001] 
Fazit:

In Abschnitt 3.5.2.3 wurde gezeigt, dass die Auswertung der vorliegenden Versuchsergebnisse in der Tendenz ähnliche Kerbfälle liefert wie sie in den Normen [DNV 2001] [BS 7608:1993] aufgeführt werden. Für Stumpfnähte mit Badsicherung liegt die Ermüdungsfestigkeit in den Versuchen des Lehrstuhls für Stahlbau ICOM über denjenigen der Grossbauteilversuche der Versuchsanstalt für Stahl, Holz und Steine der Universität Karlsruhe. Für Stumpfnähte ohne Badsicherung liegen die Resultate der Kleinbauteilversuche aus Karlsruhe hingegen über den Ergebnissen der vorliegenden Untersuchung. Die fehlerbehafteten Nähte ergeben in beiden Untersuchungen eine sehr niedrige Ermüdungsfestigkeit.

Diese Diskrepanz zeigt, dass bezüglich der Ermüdungsfestigkeit von Stumpfnähten zwischen Hohlprofilen noch Forschungsbedarf besteht. Im Rahmen eines gemeinsames Forschungsprojekts ${ }^{4}$ mit der Versuchanstalt für Stahl, Holz und Steine der Universität Karlsruhe, welches Ende 2006 abgeschlossen sein wird, wird der Einfluss der Ausbildung der Stumpfnähte auf die Ermüdungsfestigkeit eingehender untersucht.

\subsection{3 Überprüfung der Stahlgussknoten auf Gussfehler und Ermüdungsrisse}

Da während den Ermüdungsversuchen keine Risse in den Stahlgussknoten auftraten, wurden zwei willkürlich ausgewählte Knoten der Versuchsserie A und B nochmals zusätzlich zu den bereits durch die Stahlgiesserei durchgeführten Prüfungen auf vorhandene Gussfehler und eventuelle Ermüdungsrisse hin überprüft. Dazu wurden die Gussknoten zunächst in mehrere Teile zerschnitten und die Schnittflächen teilweise poliert (siehe Beispiel in Fig. 3.28). Es wurden keine visuell erkennbaren Gussfehler oder Ermüdungsrisse entdeckt.

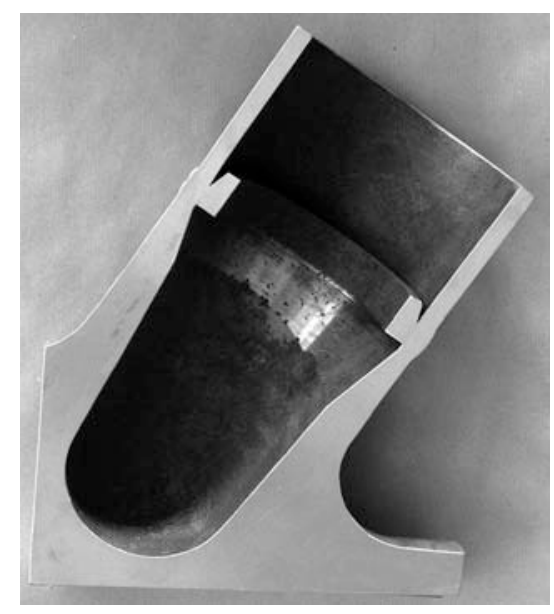

Fig. 3.28: Polierte Schnittfläche eines Knotens der Serie B

Drei Schnittteile von verschiedenen Knotenstutzen wurden anschliessend im Rahmen eines externen Auftrags mit Ultraschall überprüft [N.D.T. SA 2004] [N.D.T. SA 2004.1]. Diese Prüfungen ergaben, dass die Knotenstutzen der Gütestufe 1 und teilweise der Stufe 2 nach [DIN 1690-2:1985] entsprechen, also über der geforderte Gütestufe 3 (Tab. 3.7) liegen.

\footnotetext{
${ }^{4}$ Forschungsvorhaben der FOSTA-Forschungsvereinigung Stahlanwendung e.V. P591 „Wirtschaftliches Bauen von Strassen- und Eisenbahnbrücken mit Stahlhohlprofilen“
} 
Zusätzlich wurden aus einem Schnittteil zehn Zugproben entnommen, um eventuelle Gussfehler durch einen Sprödbruch freizulegen. Die Zugproben wurden in flüssigem Stickstoff während mindestens 10 min abgekühlt (Fig. 3.29 a)) und anschliessend in den Versuchsstand eingespannt. Die Zugproben wurden mit einer Geschwindigkeit von $2 \mathrm{~mm} / \mathrm{sec}$ belastet, um ein Aufwärmen der Probekörper während des Zugversuchs zu verhindern und einen Sprödbruch zu provozieren. Fig. 3.29 b) zeigt eine gebrochene Zugprobe. Der Sprödbruch zeichnet sich dadurch aus, dass die Bruchstelle keine Einschnürung aufweist und die Bruchflächen senkrecht zur Zugrichtung liegen.

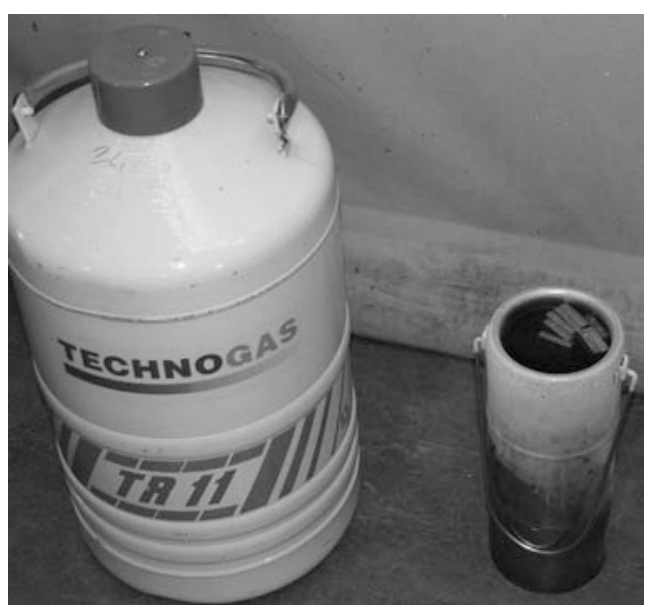

a) Abkühlung in flüssigem Stickstoff $\mathrm{N}_{2}$

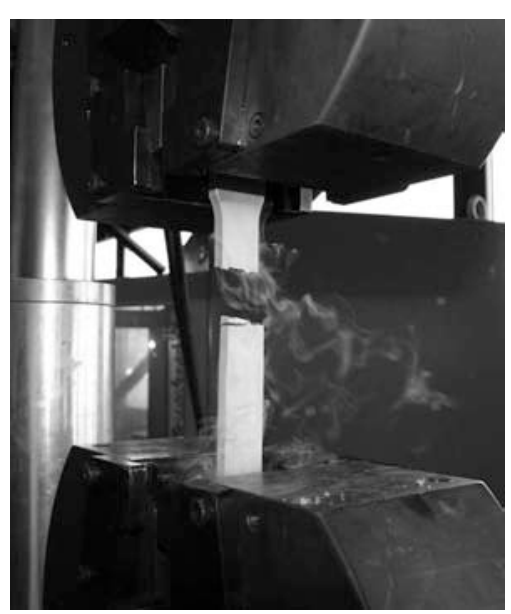

b) Gebrochene Zugprobe

Fig. 3.29: Versuchsanordnung der Zugversuche

Auf einigen Bruchflächen konnten Gussfehler ausfindig gemacht werden. Dabei handelt es sich bei den meisten Fehlern um kleine Schlackeneinschlüsse und Schlauchporen mit Schlackeneinschlüssen. In Fig. 3.30 werden drei Bruchflächen als Beispiel dargestellt. Der Probekörper 105.1 weist mehrere kleine Fehler auf; der grösste ist eine Schlauchpore mit einer Länge von ungefähr $2.7 \mathrm{~mm}$. Auf der Bruchfläche des Probekörpers 113.1 sind Schlauchporen bis zu $1.7 \mathrm{~mm}$ Länge zu sehen. Die dritte Bruchfläche zeigt einen Schlackeneinschluss mit einer Ausdehnung von $2.5 \mathrm{~mm}$ auf $1 \mathrm{~mm}$. Angaben zur Tiefenausdehnung können leider anhand der Bruchfläche nicht gemacht werden.

Die gefundenen Fehler fallen alle unter die in [Stahl-Eisen-Prüfblatt 1922:1985] definierte Registriergrenze für einen Kreisscheibenreflektor von $3 \mathrm{~mm}$ und sind somit nicht registrierpflichtig.
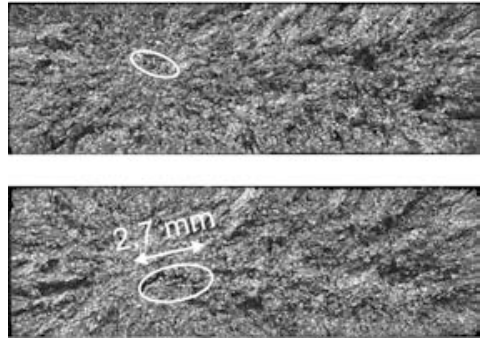

a) Probekörper 105.1

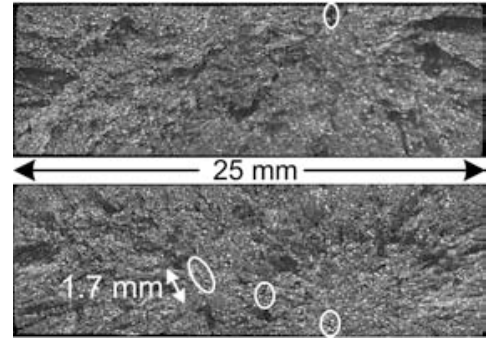

b) Probekörper 113.1

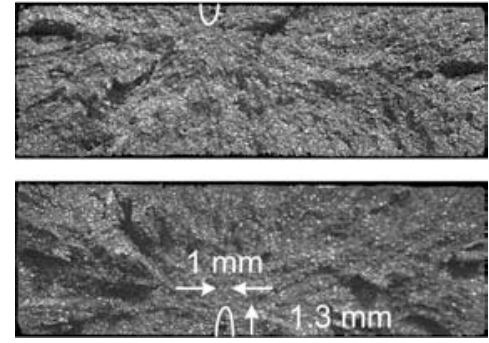

c) Probekörper 115.1

Fig. 3.30: Bruchflächen mit Gussfehlern 


\subsection{ZUSAMMENFASSUNG UND FOLGERUNGEN}

Die Auswertung der Messergebnisse unter statischer Belastung ergibt Folgendes:

- Der Anteil der Dehnung infolge des Sekundärbiegemoments $\varepsilon_{\mathrm{b}}$ an den Nominaldehnungen $\varepsilon_{\text {nom }}$ ist - im Gegensatz zur Zugdiagonale - für die Druckdiagonale und den Untergurt relativ niedrig. Eine Änderung des Sekundärbiegemoments hat somit nur in der Zugdiagonale einen grossen Einfluss auf die Nominaldehnungen $\varepsilon_{\text {nom. }}$.

- Die Verkürzung der Stutzenlängen hat zwei Auswirkungen. Einerseits verschiebt sich die Schweissnaht gegen den Achsenschnittpunkt zu, also in Richtung höherer Sekundärbiegemomente. Andererseits werden die Rundhohlprofile länger, ihre Biegesteifigkeit und damit die Sekundärbiegemomente nehmen ab. In den Diagonalen überwiegt der zweite Effekt, im Untergurt heben sich die Einflüsse gegenseitig auf.

- Die Reduktion der Steifigkeit der Rundhohlprofile bei gleich bleibender Stutzenlänge führt zu einer weiteren Abnahme der Sekundärbiegemomente.

Es wird daher empfohlen, bei der Wahl der Knotenform die Knotenstutzen mit minimaler Länge auszuführen. Dadurch werden die Sekundärbiegemomente in den Diagonalen reduziert und Herstellungskosten (Materialkosten) eingespart.

Die Auswertung der Resultate zum Risswachstum aus den Ermüdungsversuchen ergibt Folgendes:

- Die Rissinitiierung fand stets an der Wurzelseite in Schweissnahtmitte statt, die Risse breiteten sich also von der Hohlprofilinnenseite her aus. Nach dem Wanddurchbruch breiteten sie sich zunächst radial entlang der Schweissnahtmitte und im weiteren Verlauf des Risswachstums entlang des Schweissnahtfusses oder entlang der Wärmeeinflusszone auf der Hohlprofilseite aus.

- Auf Grund des schnellen Risswachstums ab Wanddurchbruch war es schwierig, dessen exakten Zeitpunkt während des Versuches festzustellen. Die Anzahl Lastwechsel ab dem Zeitpunkt der Rissentdeckung bis zum Abbruch des Versuchs bei einer Risslänge von ungefähr $50 \%$ des Hohlprofilumfangs ist im Verhältnis zur gesamten Lebensdauer sehr gering und beträgt höchstens etwa $10 \%$.

Die Versuchsergebnisse haben sehr eindeutig gezeigt, dass für die gewählten Parameter und die in den Versuchen vorliegende Konfiguration die Schweissnähte massgebend sind für die Ermüdungsfestigkeit. In den Gusskomponenten wurden keinerlei Ermüdungsrisse beobachtet. Bei der Ermittlung der Kerbfälle wurden die Spannungskonzentrationen in den Schweissnähten rechnerisch mitberücksichtigt. Die Bestimmung der Ermüdungsfestigkeit beruht auf eine sehr kleine Anzahl $(<10)$ Versuchsergebnisse. Aus diesem Grund sind weitere Versuchsergebnisse erforderlich. Die Auswertung der Resultate ergibt:

- Für die Stumpfnähte ohne Schweissbadsicherung ergibt sich eine Ermüdungsfestigkeit mit $\Delta \sigma_{\mathrm{C}}=54 \mathrm{MPa}$.

- Für die Stumpfnähte mit Schweissbadsicherung ergibt sich eine Ermüdungsfestigkeit mit $\Delta \sigma_{\mathrm{C}}=87 \mathrm{MPa}$.

- Für fehlerbehaftete Nähte ohne Schweissbadsicherung wird eine Ermüdungsfestigkeit mit $\Delta \sigma_{\mathrm{C}}=27 \mathrm{MPa}$ gemessen.

- Im Vergleich zu den Versuchsergebnissen der Grossbauteilversuche der Versuchsanstalt für Stahl, Holz und Steine an der Universität Karlsruhe ergeben die Versuche der vorliegenden Ar- 
beit für Stumpfnähte mit Badsicherung eine höhere Ermüdungsfestigkeit. Diese Tendenz wird in bestehenden Normen bestätigt.

Mit dem Nachweis von vorhandenen Ungänzen wird im Anschluss an die Ermüdungsversuche gezeigt, dass die Gusskomponenten von sehr guter Qualität waren und keine wesentlichen Ungänzen aufwiesen. Nachträglich durchgeführte Ultraschalluntersuchungen und Zugversuche an aus den getesteten Stahlgussknoten ausgearbeiteten Kleinproben zeigen:

- Die untersuchten Knotenstutzen entsprechen der Gütestufe 1 und teilweise der Stufe 2 nach [DIN 1690-2:1985], liegen also über der geforderten Gütestufe 3.

- Auf den Bruchflächen der Zugproben konnten mit einem Sprödbruch Gussfehler freigelegt werden. Dabei handelt es sich um Schlackeneinschlüsse, Gasblasen und Schlauchporen mit Schlackeneinschlüssen. Die Grösse der Fehler liegt unter der Registriergrenze von 3 mm für Ultraschalluntersuchungen nach [Stahl-Eisen-Prüfblatt 1922:1985].

Die Versuchsergebnisse haben sehr eindeutig gezeigt, dass für die vorliegende Konfiguration der Versuchskörper die Stumpfschweissnähte für die globale Ermüdungsfestigkeit massgebend sind. Weil für eine wirtschaftlich optimal Bemessung der Stahlgussknoten ihr Ermüdungswiderstand demjenigen der Schweissnahtanschlüsse entsprechen soll, werden sich die weiteren numerischen und analytischen Untersuchungen auf die Ermüdungsfestigkeit der Stahlgussknoten konzentrieren. Der Einfluss der Ausbildung der Stumpfschweissnähte auf die Ermüdungsfestigkeit wird zeitgleich mit der vorliegenden Arbeit im Rahmen eines gemeinsamen Forschungsprojekts ${ }^{5}$ mit der Versuchanstalt für Stahl, Holz und Steine der Universität Karlsruhe, welches Ende 2006 abgeschlossen sein wird, eingehender untersucht.

\footnotetext{
${ }^{5}$ Forschungsvorhaben der FOSTA-Forschungsvereinigung Stahlanwendung e.V. P591 „Wirtschaftliches Bauen von Strassen- und Ei-
} senbahnbrücken mit Stahlhohlprofilen“ 



\section{MODELLBILDUNG VON STAHLGUSSKNOTEN}

\subsection{VORGEHEN}

Die im Kapitel 3 beschriebenen experimentellen Untersuchungen geben Auskunft über den Dehnungs- und Spannungsverlauf unter statischer Last in den Stahlgussknoten und den anschliessenden Hohlprofilen. Dabei zeigt sich, dass das Ermüdungsverhalten der Probekörper massgeblich durch die Ermüdungsfestigkeit der Schweissnähte zwischen den Stahlgussknoten und den anschliessenden Rundhohlprofilen beeinflusst wird. Sowohl zerstörungsfreie als auch zerstörende Untersuchungen an getesteten Stahlgussknoten bestätigen deren sehr gute und wesentlich über der bestellten liegende Herstellungsqualität. Es können keine für das Ermüdungsverhalten relevanten Gussfehler festgestellt werden, was auch die hohe Ermüdungsfestigkeit des Stahlgusses erklärt.

Um nun trotz fehlender Versuchsergebnisse die maximal zulässigen Anfangsgrössen von Gussfehlern in Abhängigkeit der erforderlichen Ermüdungsfestigkeit eines Stahlgussknotens definieren zu können, wird ein auf der Randelementmethode (Boundary Element Method, BEM) basierendes numerisches Modell des Knotens erstellt. So können Gussfehler im Stahlgussknoten eingefügt und ihr Wachstum simuliert werden. Die Anwendbarkeit des BE-Modells wird einerseits anhand des Spannungsverlaufs im Stahlgussknoten und andererseits durch Nachrechnung des in den Versuchen beobachteten Risswachstums in den Schweissnähten überprüft.

Zur Überprüfung des Spannungsverlaufs im BE-Modell werden Ergebnisse von Berechnungen mit der Methode der finiten Elemente (Finite Element Method, FEM) und der in den Versuchen gemessene Dehnungs- bzw. Spannungsverlauf verwendet. Da das FE-Knotenmodell direkt in einen Fachwerkträger eingebunden werden kann, werden die Lagerungsbedingungen des Stahlgussknotens im Probekörper wirklichkeitsnah simuliert und die an den Stahlgussknoten angreifenden Schnittkräfte können berechnet werden. Die Software für die Randelementmethode erlaubt keine Lagerung des BE-Knotenmodells in einem Fachwerkträger, sondern nur die direkte Lagerung eines isolierten Knotenmodells. Aus diesem Grund werden für das BE-Modell die an den Knoten angreifenden Schnittkräfte aus dem FE-Modell übernommen und die Spannungen mit den FEBerechnungen und den Versuchsergebnissen validiert.

Das in den Versuchen beobachtete Risswachstum in den Schweissnähten wird mittels BEBerechnungen simuliert, um die Anwendbarkeit der BE-Methode für das Risswachstum zu überprüfen.

\subsection{MODELLIERUNG MIT FINITEN ELEMENTEN (FE)}

\subsubsection{Besonderheiten}

Die Stahlgussknoten weisen bedingt durch die Ausrundungen zwischen den Diagonal- und Untergurtstutzen eine komplizierte, dreidimensionale Geometrie auf (Fig. 3.2). Für die exakte Modellierung der Knotengeometrie wird daher auf Grund seiner intelligenten Werkzeuge der Preprozessor des kommerziell erhältlichen Finite Element Programms I-Deas [SDRC 1999] herangezogen. Damit ist der Verschnitt der Vollprofile der Diagonalen mit demjenigen des Untergurts, das Aushöhlen der Stutzen und das Verlegen der Ausrundungen zwischen den Diagonalen sowie zwischen den Diagonalen und dem Untergurt möglich. Auf Grund der Längssymmetrie der Stahlgussknoten 
wird nur eine Längshälfte des Knotens modelliert. Fig. 4.1 und Fig. 4.2 zeigen die Innen- und Aussenansicht des FE-Knotenmodells mit der Geometrie der Serie A, wobei der dunkel eingefärbte Bereich den Stahlgussknoten und die heller eingefärbten Bereiche die anschliessenden Hohlprofile darstellen. Letztere werden auf Grund des Steifigkeitssprungs zwischen den Knoten und den Hohlprofilen ebenfalls modelliert. Bei den Schweissnähten der Stahlgussknoten der Serie A wird der Wanddickensprung zwischen Hohlprofil und Knotenstutzen linear über eine Länge von $10 \mathrm{~mm}$ verlaufend realisiert, die Details der in Fig. 3.5 dargstellten Geometrie werden nicht berücksichtigt.

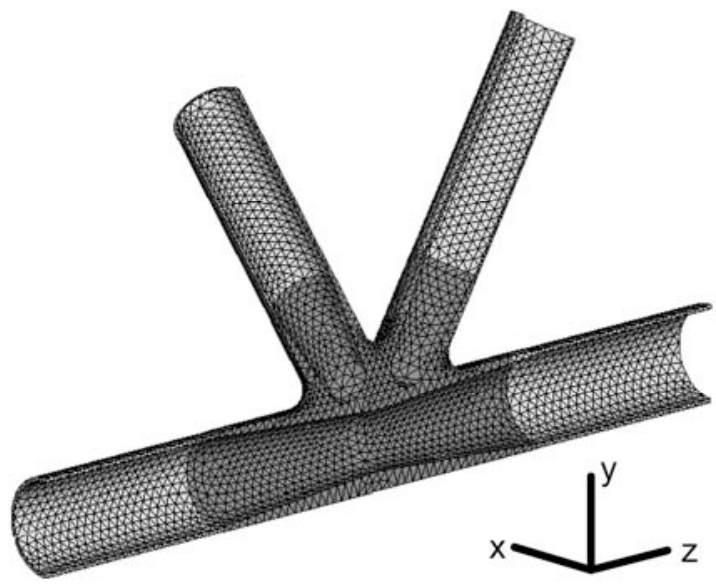

Fig. 4.1: FE-Modell Serie A, Innenansicht

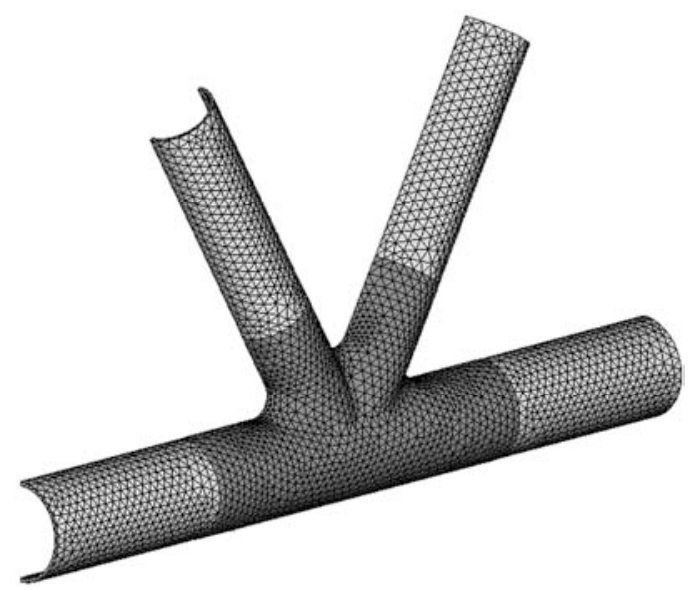

Fig. 4.2: FE-Modell Serie A, Aussenansicht

Auf Grund der komplizierten Geometrie kann das Knotenmodell nur frei mit tetraederförmigen Volumenelementen vernetzt werden. In den Ausrundungen in der $y z$-Ebene, wo Spannungskonzentrationen zu erwarten sind, wird die Anzahl der Elementkanten auf 10 festgelegt, da durchgeführte Berechnungen ergaben, dass ab 10 Elementen keine bessere Genauigkeit mehr resultiert.

Die FE-Berechnungen werden mit dem kommerziellen Finite Elemente Programm ABAQUS [ABAQUS 2000] durchgeführt. Für die Vernetzung wird das quadratische (zweiter Ordnung), 10Knoten ABAQUS Volumenelement C3D10 gewählt, weil es im Vergleich zu gleichgrossen linearen Volumenelementen insbesondere bei den Ausrundungen eine bessere Volumenanpassung und genauere Resultate liefert. Da eine linear elastische Berechnung durchgeführt wird, müssen nur der Elastizitätsmodul $E$ und die Querdehnungszahl $v$ festgelegt werden. Es kommen die Werte $E=210000 \mathrm{MPa}$ und $v=0.3$ zur Anwendung.

Das Knotenmodell wird analog zu den experimentell untersuchten Probekörpern in einen Fachwerkträger eingebunden (Fig. 4.3). Die Fachwerkstäbe werden mit 3-Knoten Balkenelementen mit quadratischer Interpolation (B32) modelliert. Der Fachwerkträger ist einfach gelagert und in Feldmitte mit einer Einzelkraft $Q$ belastet. Für eine Längshälfte des Knotens entspricht die Einzelkraft der Hälfte der maximalen Ermüdungslast $Q=0.5 \cdot Q_{\max }=500 \mathrm{kN}$. Lediglich der erste Ring von Volumenelementen unterliegt durch die Überleitung der Schnittkräfte von den Balkenelementen zum Knotenmodell an den Hohlprofilenden einer überhöhten Verformung, so dass keine weiteren Anpassungen der Randbedingungen der Hohlprofilenden nötig sind. Fig. 4.4 zeigt die für die Symmetrieebene $X=0$ des Knotenmodells erforderlichen Randedingungen: die Verschiebung $u$ in $x$ Richtung sowie die Rotationen $\Phi_{2}$ und $\Phi_{3}$ um die $y$ bzw. $z$-Achse werden in den auf der $y z$-Ebene (dunkel eingefärbt) liegenden Knoten blockiert. 


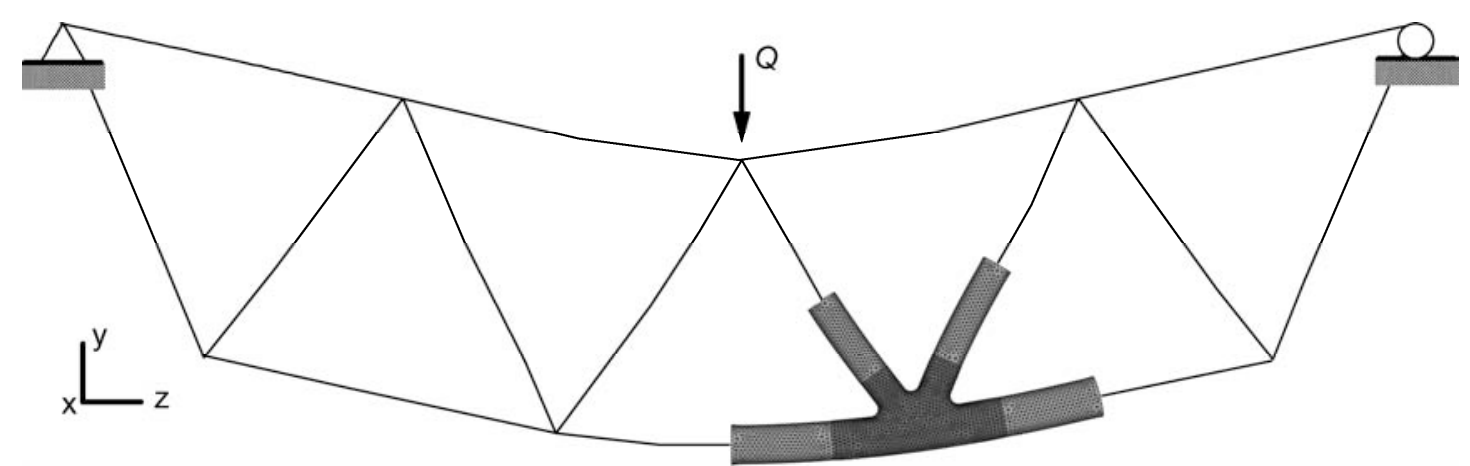

Fig. 4.3: FE-Modell, in Stabfachwerkträger eingebunden

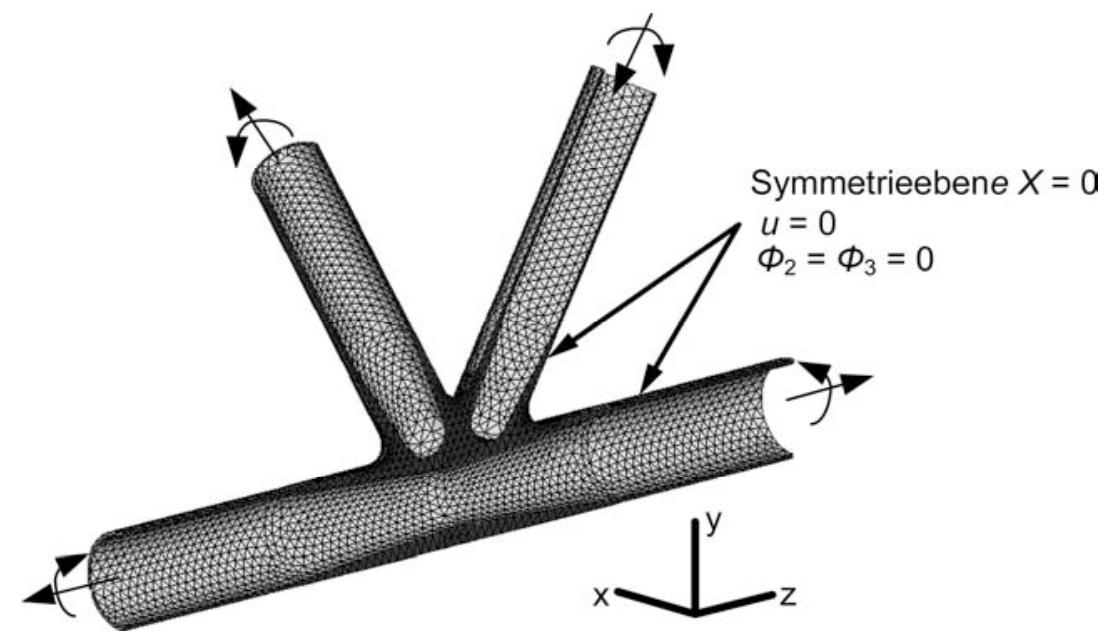

Fig. 4.4: Randbedingungen des isolierten FE-Modells

\subsubsection{Validierung}

Die Validierung des FE-Modells bezieht sich auf das vorangehenden Abschnitt beschriebene Knotenmodell für die Serie A und besteht im Wesentlichen darin, das simulierte Verhalten des im Fachwerkträger eingebunden Stahlgussknotens unter statischer Last mit den Messdaten zu vergleichen. Die zum Vergleich herangezogene Grösse ist der Dehnungs- bzw. Spannungsverlauf in der $y z$-Ebene im Stahlgussknoten und in den anschliessenden Hohlprofilen.

Fig. 4.5 zeigt den Verlauf der maximalen und minimalen Hauptspannungen zwischen den beiden Diagonalen. Es ist zu erkennen, dass in der Druckdiagonale die minimalen und in der Zugdiagonale die maximalen Hauptspannungen vorherrschend sind. Auf Grund des im Wesentlichen einaxialen Spannungszustands können die Messdaten zum Dehnungsverlauf (Fig. 3.14) mit dem Hookschen Gesetz $\sigma=\varepsilon \cdot E$ in Hauptspannungen umgerechnet werden.

Die Spannungen in den Hohlprofilen (hell eingefärbter Bereich des dargestellten Knotens) variieren linear. Da die Normalkraft in den Fachwerkstäben konstant ist, lässt dies auf das Vorhandensein von Sekundärmomente schliessen.

Beim Übergang vom Gussknoten zu den Hohlprofilen steigen die Spannungen auf Grund des Wanddickensprungs (Vergleiche Tab. 3.7) an. Da das FE-Modell nicht für die präzise Simulation des Spannungsverlaufs nahe der Schweissnaht ausgelegt wurde, sind hier Abweichungen zwischen Messwerten und Berechungen vorhanden. 


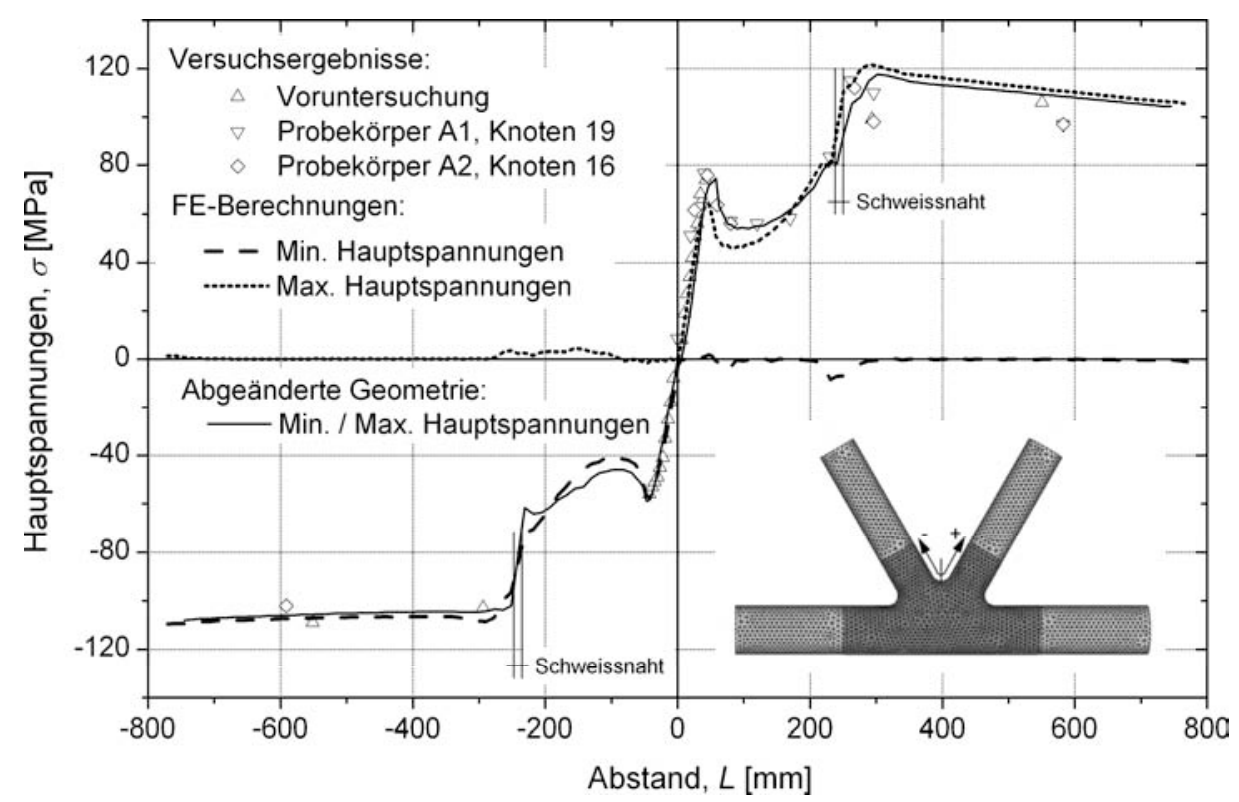

Fig. 4.5: Hauptspannungsverlauf zwischen den Diagonalen, Messungen und Berechnungen, Serie A

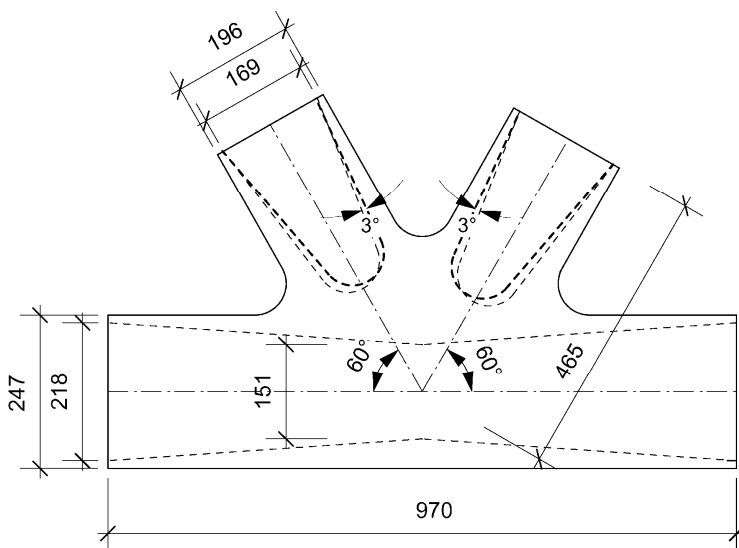

a) Verdrehung der Innenhohlräume der Diagonalstutzen

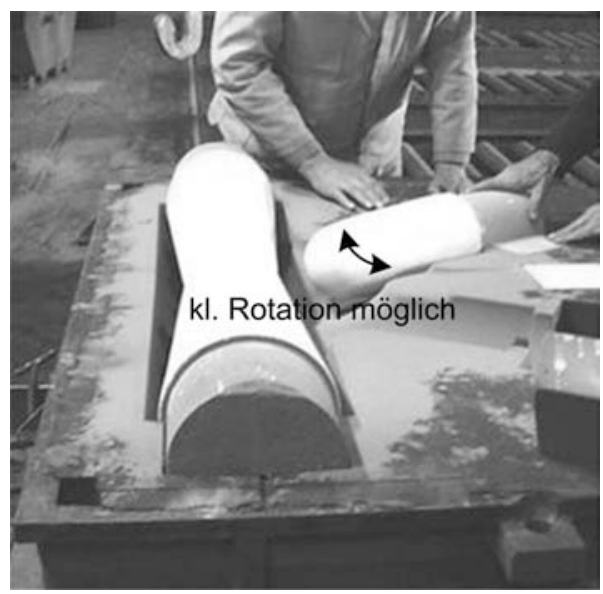

b) Mögliche Rotation der Kernstücke, die die Hohlräume der Diagonalen bilden

Fig. 4.6: Abgeänderte Knotengeometrie, Knoten Serie A

Der rechnerische Spannungsverlauf zeigt in der Ausrundung zwischen den Diagonalen Spannungskonzentrationen, die auch mit den Dehnmessstreifen gemessen wurden. Allerdings liegen im Bereich der Spannungskonzentration die Hauptspannungen aus den Versuchen über den Berechnungen. Untersuchungen haben gezeigt, dass bereits bei einer leichten Verdrehung der Innenhohlräume um $3^{\circ}$ (Fig. 4.6) der rechnerische Spannungsverlauf an der Spitze um ungefähr $30 \%$ steigt und nun weitgehend dem Spannungsverlauf aus den Versuchen entspricht. Eine Verdrehung der Hohlräume kann beim Giessvorgang eintreten, da die Kernstücke nur am Stutzenende fixiert werden können (Fig. 4.6 b).

Als weiterer Vergleich wird in Fig. 4.7 der Hauptspannungsverlauf auf der Unterseite des Untergurtstutzens wiedergegeben. Auch hier liegt näherungsweise ein einaxialer Spannungszustand vor und die Dehnungen aus Fig. 3.15 können mit dem Hookschen Gesetz in Spannungen umgerechnet werden. Im Knotenbereich und in den Hohlprofilen ist die Übereinstimmung sehr gut. 


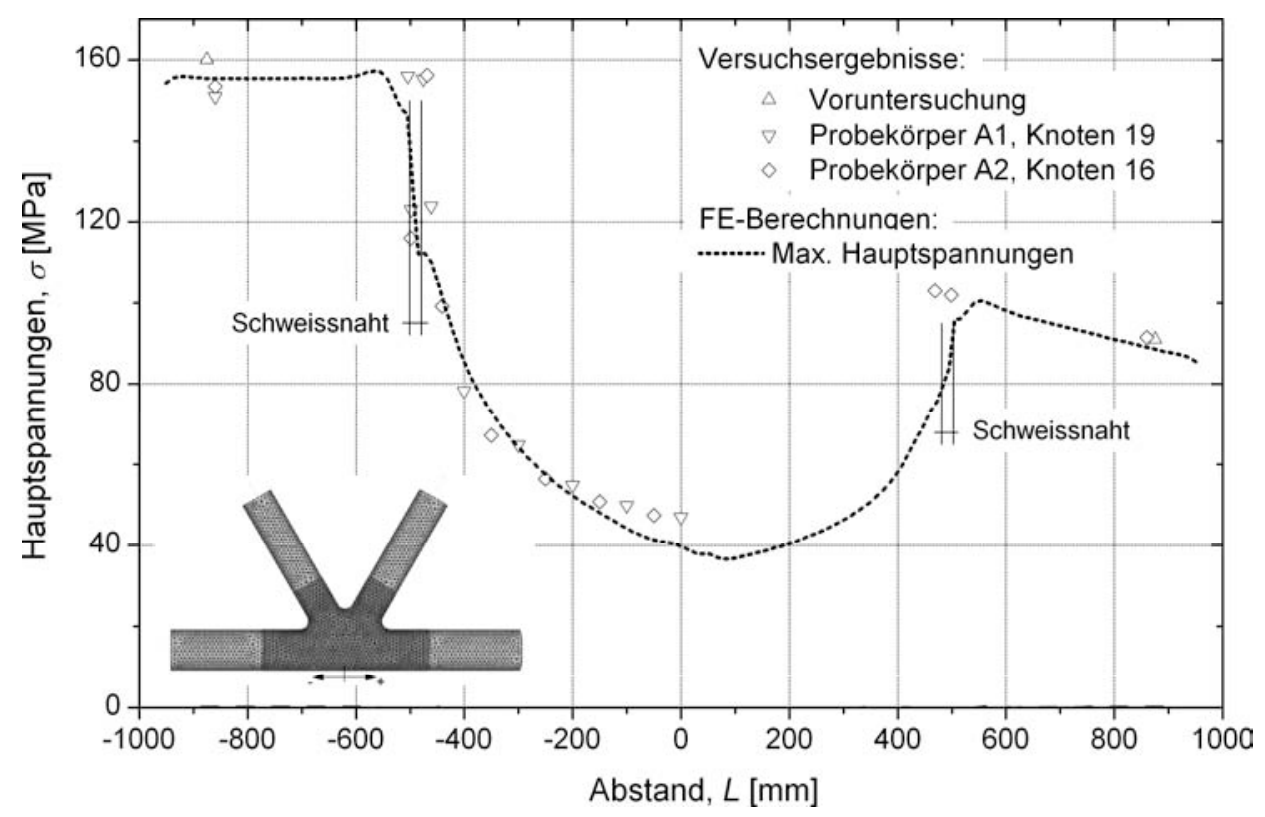

Fig. 4.7: Hauptspannungsverlauf auf der Unterseite des Untergurts, Messungen und Berechnungen, Serie A

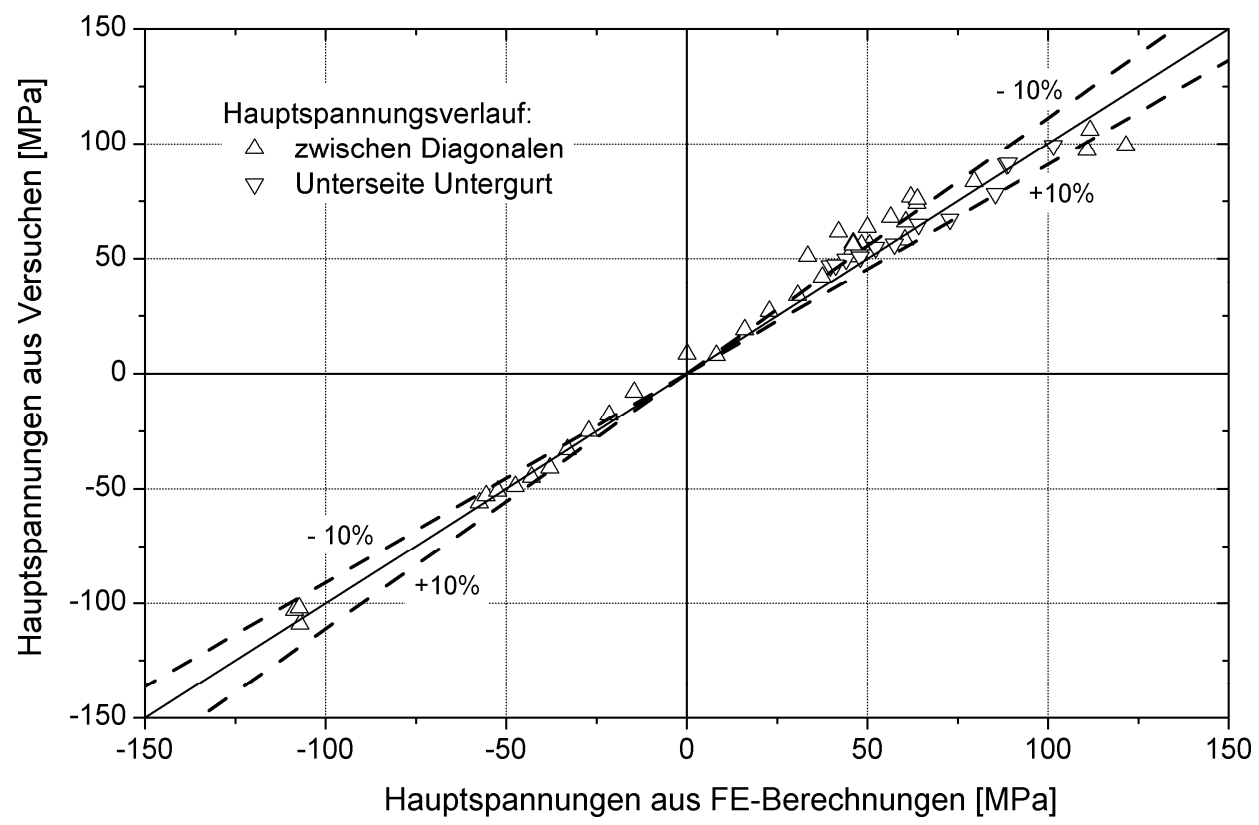

Fig. 4.8: Hauptspannungen aus FE-Berechnungen zu Hauptspannungen aus Versuchen

Abschliessend werden in Fig. 4.8 für alle beschriebenen Spannungsverläufe die Hauptspannungen aus den FE-Berechnungen den Versuchswerten gegenübergestellt. Die Schweissnahtbereiche werden in Fig. 4.8 nicht mit aufgenommen, weil das Knotenmodell wie erwähnt nicht für ihre präzise Simulation ausgelegte wurde. Obwohl für die meisten Wertepaare die Abweichungen unter $\pm 10 \%$ liegen, kommen in der Ausrundung zwischen den beiden Diagonalen auch grössere Abweichungen vor. Der Grund dafür dürfte die Abweichung der tatsächlichen von der nominellen Geometrie der Stahlgussknoten sein. 


\subsection{MODELLIERUNG MIT RANDELEMENTEN (BE)}

\subsubsection{Die Methode der Randelemente}

Der wesentliche Unterschied der Randelementmethode im Vergleich zur Methode der Finiten Elemente besteht darin, bei einem Modell lediglich dessen Rand und nicht dessen Inneres zu diskretisieren. Dreidimensionale Körper werden mit Flächenelementen diskretisiert, bei zweidimensionalen Problemen umranden Linienelemente das Modell. Die Randelementmethode reduziert also das Elementnetz und damit das zu lösende Gleichungssystem um eine Dimension. Die Generierung eines Modells ist übersichtlicher und insbesondere Risswachstumssimulationen sind wesentlich einfacher zu handhaben. Das Prinzip der Randelementmethode wird beispielsweise in [Hartmann 1989] umfassend beschrieben und soll nachfolgend nur in den Grundzügen erläutert werden.

Die einfachste Anwendung des Prinzips der Randelemente ist eine lineare Funktion, deren Randwerte bekannt sind. Diese Randwerte bestimmen den Verlauf der Funktion, da dieser durch Einsetzen der Randwerte in die Gleichung der linearen Funktion eindeutig bestimmt werden kann. Die Randwerte bei einem Bauteil sind die Endverschiebungen und Endkräfte. Sind alle Endverschiebungen und -kräfte sowie die äussere Belastung auf dem Rand eines Bauteils bekannt, können die Dehnungen und Spannungen im Inneren des Bauteils eindeutig bestimmt werden, wenn die jeweilige so genannte Einflussfunktion bekannt ist. Auf Grund von Lagerbedingungen kann aber nur ein Teil der Endverschiebungen und -kräfte ohne Rechenaufwand bestimmt werden, die übrigen Randwerte sind unbekannt. Die unbekannten Randwerte können mittels Kopplungsbedingungen auf dem Rand bestimmt werden, die mit Hilfe des Satzes von Betti aufgestellt werden. Dieser besagt, dass die Verschiebungsarbeit im Knoten i eines Bauteils infolge einer Einheitskraft in Knoten $k$ gleich der Verschiebungsarbeit im Knoten $k$ infolge einer Einheitskraft im Knoten $i$ ist:

$$
A_{i, k}=A_{k, i}
$$

Mit anderen Worten sind die gegenseitigen äusseren Arbeiten zweier Gleichgewichtszustände gleich gross. Die Anzahl der unbekannten Randwerte bestimmt die Anzahl der nötigen Gleichgewichtszustände, die zur Lösung der unbekannten Randwerte notwendig sind. Steht diese Anzahl Gleichgewichtszustände zur Verfügung, kann das Gleichungssystem gelöst werden. Da der Satz von Betti auf linearen Zusammenhängen beruht, ist das Anwendungsgebiet der Randelementmethode auf linear elastische Probleme beschränkt.

Für die elementarsten statischen Systeme können die Gleichgewichtszustände noch relativ einfach aufgestellt werden, bei mehrdimensionalen Problemen wird dies jedoch sehr aufwendig. Daher wird die benötigte Anzahl von Gleichgewichtszuständen aus der so genannten Grundlösung - der Verformungsfigur eines unendlichen elastischen Mediums unter dem Angriff einer Einzelkraft entnommen. Mit der Grundlösung steht ein unendlicher Vorrat an Gleichgewichtszuständen zur Verfügung. Nachdem alle Grössen sortiert sind, ergibt sich letztendlich die folgende Formulierung des Gleichungssystems:

$$
[\mathrm{K}] \cdot \overrightarrow{\mathrm{u}}=\overrightarrow{\mathrm{f}}+\overrightarrow{\mathrm{p}}
$$

[K] Steifigkeitsmatrix mit der Dimension $k \times k$

$\overrightarrow{\mathrm{u}} \quad$ Vektor der Endverschiebungen

$\overrightarrow{\mathrm{f}} \quad$ Vektor der Endkräfte

$\overrightarrow{\mathrm{p}} \quad$ Festhaltekräfte; $\overrightarrow{\mathrm{p}}=0$ wenn die äusseren Kräfte nur an den Enden angreifen 
Nach dem Einsetzen der durch die Lagerbedingungen bekannten Endverschiebungen und -kräfte in die Gleichungen (4.2) können die unbekannten Randwerte berechnet werden. Sind alle Randwerte bekannt, ist wie eingangs erwähnt die Einflussfunktion vollständig definiert.

Im Gegensatz zur Methode der Finiten Elemente ist die Steifigkeitsmatrix der Randelementmethode voll besetzt, was einen erhöhten Rechenaufwand zur Lösung des Gleichungssystems zur Folge hat. Dies wird jedoch teilweise durch die kleinere Dimension der Steifigkeitsmatrix kompensiert.

Das in der vorliegenden Arbeit verwendete kommerziell erhältliche Programm BEASY [BEASY 2003] basiert auf der Randelementmethode. Es ist insbesondere für Ermüdungs- und Rissfortschrittsuntersuchungen, allgemeine mechanische und akustische Probleme sowie für Berechnungen zur durch elektrische und magnetische Felder induzierten Korrosion geeignet. Entwicklung und Forschung werden in enger Zusammenarbeit mit dem Wessex Institute of Technology (WIT) realisiert, welches weltweit für seine Forschung und seine Leistungen im Gebiet der Technologie und Entwicklung von Software für die Anwendung der Randelementmethode bekannt ist.

Für dreidimensionale Rissfortschrittsuntersuchungen kommt im Programm BEASY die duale Randelementmethode zur Anwendung. Dank der dualen Randelementmethode kann ein Riss mittels einer mit Elementen vernetzte Fläche (im Falle eines zweidimensionalen Problems mit einer Linie) modelliert werden. Da sich ausser auf der Rissfläche keine Elemente im Modellinnern befinden, entfällt die bei FE-Modellen nötige aufwendige Anpassung der Vernetzung in der Rissumgebung. Die zweite Rissfläche wird automatisch mit „dualen“, die Netzpunkte der ersten Rissfläche benutzenden, Elementen vernetzt. Im Rahmen der vorliegenden Arbeit wird die duale Randelementmethode nicht erläutert, weitere Informationen können der spezialisierten Literatur [Mi et al. 1992] [Portela et al. 1993] [Mi et al. 1994] entnommen werden. Die duale Randelementmethode wird auch für das Risswachstum von mehreren Rissen [Cisilino et al. 2004] angewandt.

Das Programm BEASY benutzt eine automatische Rissfortschrittsberechnung zur Ermittlung der Spannungsintensitätsfaktoren (SIF). Mit dieser Methode werden die Initialrisse inkrementell ausgedehnt und die SIF für jedes Inkrement bestimmt. Zur Ermittlung der SIF an der Rissfront wird das crack opening displacement Konzept herangezogen [BEASY 2003]. Die Risswachstumsrichtung wird mit dem minimum strain energy density Kriterium bestimmt [Sih et al. 1974]. Dieses Kriterium besagt, dass sich jeder Punkt auf der Rissfront immer in diejenige Richtung ausdehnt, in der die Dehnungsenergiedichte $S$, die auf einer kugelförmigen, den Punkt umgebenden Oberfläche definiert ist, ihr Minimum $S_{\min }$ annimmt. Zur Rissausdehnung kommt es jedoch nur dann, wenn die kleinste Dehnungsenergiedichte $S_{\text {min }}$ einen kritischen Wert $S_{\text {krit }}$ erreicht. Die Rissausdehnungslänge $\Delta a$ entlang der Rissfront wird proportional zu $S_{\min }$ angenommen. Somit ist entlang der neuen Rissfront nicht die Rissausdehnung sondern das Verhältnis $S_{\min } / \Delta a$ konstant. Demnach erreicht die Ausdehnungslänge $\Delta a$ ihr Maximum $\Delta a_{\max }$ im selben Punkt entlang der Rissfront, wo auch $S_{\min }$ am grössten ist. Da das Programm BEASY als maximale Ausdehnung $\Delta a_{\max }$ die Elementlänge an der Rissspitze auswählt, wird der Riss von der Rissspitze aus jeweils um eine Elementlänge verlängert.

Das Programm BEASY wurde im Bereich der Ermüdungs- und Rissfortschrittsuntersuchungen zum Beispiel im Schiffbau [Fricke et al. 2004], im Schienenbau [Mellings et al. 2005] und in der Forschung zur Vermeidung von Sprödbruch [Kühn 2005] bereits erfolgreich angewendet. 


\subsubsection{Besonderheiten}

Wie bereits für die Erstellung des FE-Modells im Abschnitt 4.2.1 beschrieben, wurde auch für das BE-Knotenmodell die Geometrie mit Hilfe des Preprozessors des kommerziellen Finite Element Programms I-Deas [SDRC 1999] erstellt und anschliessend in BEASY [BEASY 2003] importiert. Fig. 4.9 und Fig. 4.10 zeigen die Innen- und Aussenansicht des BE-Modells mit der Knotengeometrie der Serie A. Im Gegensatz zum FE-Modell werden die anschliessenden Hohlprofile hier nicht modelliert, die Schnittkräfte am Übergang zwischen Knoten und Hohlprofilen werden aus dem FEModell gelesen und direkt an den Knotenstutzen als Spannungen angesetzt. Hingegen wird auch hier die Längssymmetrie ausgenutzt und nur eine Längshälfte des Knotens modelliert.

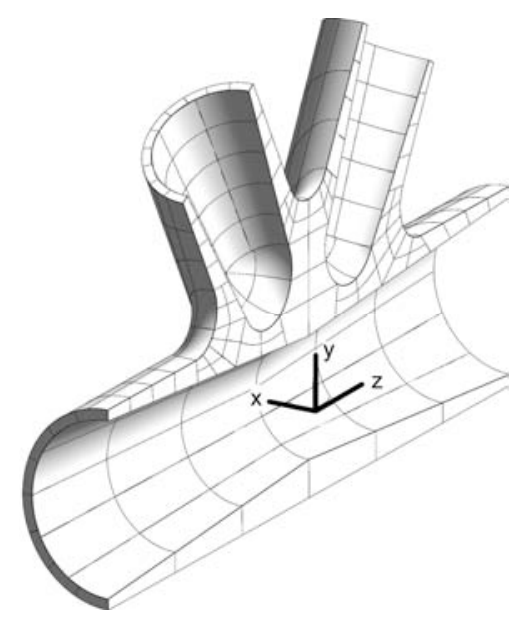

Fig. 4.9: BE-Modell Serie A, Innenansicht

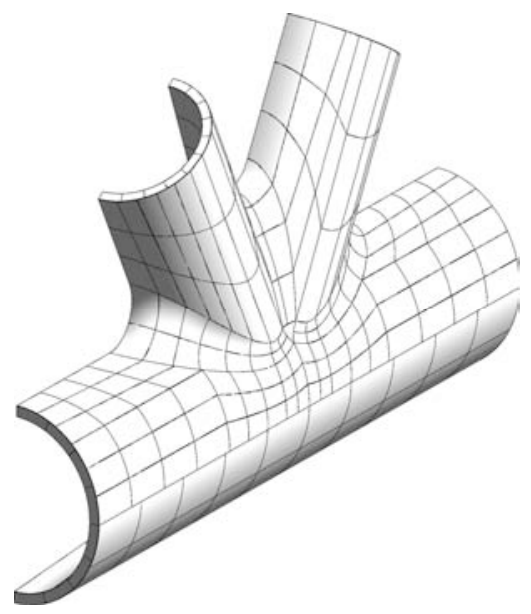

Fig. 4.10: BE-Modell Serie A, Aussenansicht

Das BE-Knotenmodell muss nur auf der Oberfläche mit Elementen vernetzt werden. Um die Vernetzung mit Flächenelementen kontrolliert durchführen zu können, wird die Oberfläche in geometrisch bedingte, grössere oder kleinere vierseitige Teilflächen unterteilt. Diese Teilflächen werden anschliessend manuell mit vierseitigen Flächenelementen Q38 mit reduzierter quadratischer Ordnung vernetzt. Diese Elemente besitzen neun in einem 3 x 3 Gitter angeordnete Netzpunkte, mit denen die Position und die Krümmung des Elements definiert werden. Die Elementgeometrie wird also durch eine quadratische Funktion angenähert.

Die Netzpunkte können unterschiedlich mit Knotenpunkten besetzt sein, welche die Verschiebungen in $x$-, $y$-, und $z$-Richtung $(u, v, w)$ als Freiheitsgrade haben. Die Spannungen werden an den Knotenpunkten berechnet. Mit neun Knotenpunkten, wenn also jeder Netzpunkt mit einem Knotenpunkt besetzt ist, ist das Element von quadratischer Ordnung. Sind nur die Elementecken mit Knotenpunkten besetzt, handelt es sich um ein Element von linearer Ordnung. Dem Element mit reduzierter quadratischer Ordnung fehlt die Besetzung des mittleren Netzpunktes. Da die Rechenzeit proportional zum Quadrat der Knotenpunkte ist, wird durch eine reduzierte Ordnung der Elemente die Rechenzeit wesentlich verkürzt.

Wenn bei zwei angrenzenden Elementen dieselben auf der Kante liegenden Knotenpunkte benutzt werden, spricht man von kontinuierlichen Knoten. Ist dies nicht der Fall, handelt es sich um diskontinuierliche Knotenpunkte. Der Vorteil der diskontinuierlichen Knotenpunkte besteht darin, dass die Kantenlängen der angrenzenden Elemente nicht übereinstimmen müssen. Dies reduziert 
den Aufwand für die Vernetzung des Modells erheblich, insbesondere wenn bei einer in einem Bereich des Modells kleineren Vernetzung kein kontinuierliche Übergang zu grösseren Elementen eingehalten werden muss, wie es im Falle eines FE-Modells der Fall wäre. So kann zum Beispiel der Einfluss der Vernetzung in den Ausrundungen zwischen den Knotenstutzen auf die Genauigkeit der Rechenergebnisse untersucht werden, indem nur sehr lokal die Elementdichte verändert wird und ohne dass das angrenzende Elementnetz angepasst werden muss. Der Nachteil der diskontinuierlichen Knotenpunkte ist die grosse Anzahl von Knotenpunkten, die die Rechenzeit erheblich verlängert.

Für linear elastische Berechnung wurde der Elastizitätsmodul $E$ und die Querdehnungszahl $v$ mit den Werten $E=210000 \mathrm{MPa}$ und $v=0.3$ festgelegt.

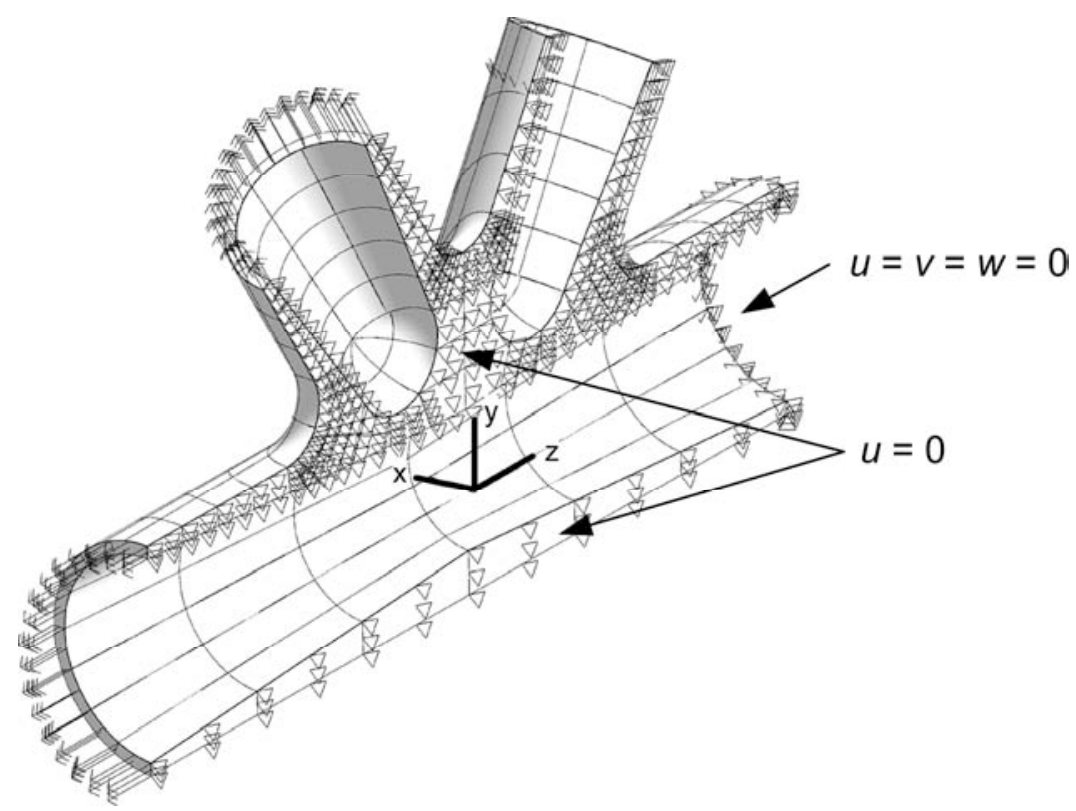

Fig. 4.11: Randbedingungen des BE-Knotenmodells

Wie bereits erwähnt, kann das BE-Knotenmodell nicht analog zu den experimentell untersuchten Probekörpern in einen Fachwerkträger eingebunden werden, da es in der Randelementmethode keine Balkenelemente gibt. Aus diesem Grund wird im BE-Modell der isolierte Knoten analysiert. Fig. 4.11 zeigt die für die Symmetrieebene des Knotenmodells erforderlichen Lagerungsbedingungen. Die Verschiebung in $x$-Richtung wird blockiert $(u=0)$, die Einspannung des Untergurtstutzens wird die durch die Blockierung der Verschiebungen in $x$-, $y$-, und $z$-Richtung $(u=v=w=0)$ erreicht. Die an die Knotenstutzen angreifenden Schnittkräfte werden als Spannungen auf die Flächen der Stutzenenden aufgebracht.

\subsubsection{Validierung}

\subsubsection{Vorgehen}

Die Validierung des BE-Modells bezieht sich auf das im vorangehenden Abschnitt beschriebene Knotenmodell für die Serie A und besteht im Wesentlichen darin, das simulierte Verhalten unter statischer Last mit den Resultaten des bereits validierten FE-Modells und den Messdaten zu vergleichen. Da die Querkräfte im Verhältnis zu den Axialkräften sehr klein sind, jedoch geometrie- und 
softwarebedingt mit hohem Aufwand ins Modell eingetragen werden müssten, werden sie gleich null gesetzt. Die Werte der Axialkräfte und Biegemomente werden leicht angepasst, um das Gleichgewicht des Knotens weiterhin zu garantieren.

Die von den Probekörpern abweichenden Lagerungsbedingungen des BE-Modells müssen kontrolliert werden. Die Kontrolle der Lagerungsbedingungen ist auch im Hinblick auf die weiteren Untersuchungen im Kapitel 5 wichtig, wo die Stahlgussknoten als Teil einer Fachwerkbrücke untersucht werden. Die zum Vergleich herangezogene Grösse ist der Dehnungs- bzw. Spannungsverlauf in der $y z$-Ebene des Knotens.

Abschliessend wird das in den experimentellen Untersuchungen beobachtete Risswachstum in den Stumpfschweissnähten mit dem Programm BEASY simuliert und mit den Messdaten verglichen, um die Plausibilität der Rechenergebnisse zu überprüfen.

\subsubsection{Spannungen im Knoten}

Das BE-Modell wird überprüft, indem es mit den Versuchsergebnissen aus der Serie A und den Berechnungen aus dem FE-Modell verglichen wird. Für den Spannungsverlauf zwischen den Diagonalen zeigt Fig. 4.12 eine gute Übereinstimmung. Die Hauptspannungen aus den BE-Berechnungen sind nur bis zu den Stutzenenden dargestellt, weil das BE-Modell nur den Stahlgussknoten ohne anschliessende Hohlprofile berücksichtigt.

Die zwei Spannungskonzentrationen in der Ausrundung zwischen den Diagonalen werden im BEModell gut wiedergegeben. Untersuchungen haben gezeigt, dass ab 20 Elementen entlang der Ausrundung zwischen den Diagonalen die Genauigkeit der Resultate nicht mehr zunimmt.

Fig. 4.13 zeigt den Spannungsvergleich auf der Unterseite des Untergurtstutzens. Auch hier ergibt sich eine gute Übereinstimmung zwischen dem FE-Modell, dem BE-Modell und den Versuchsergebnissen. 


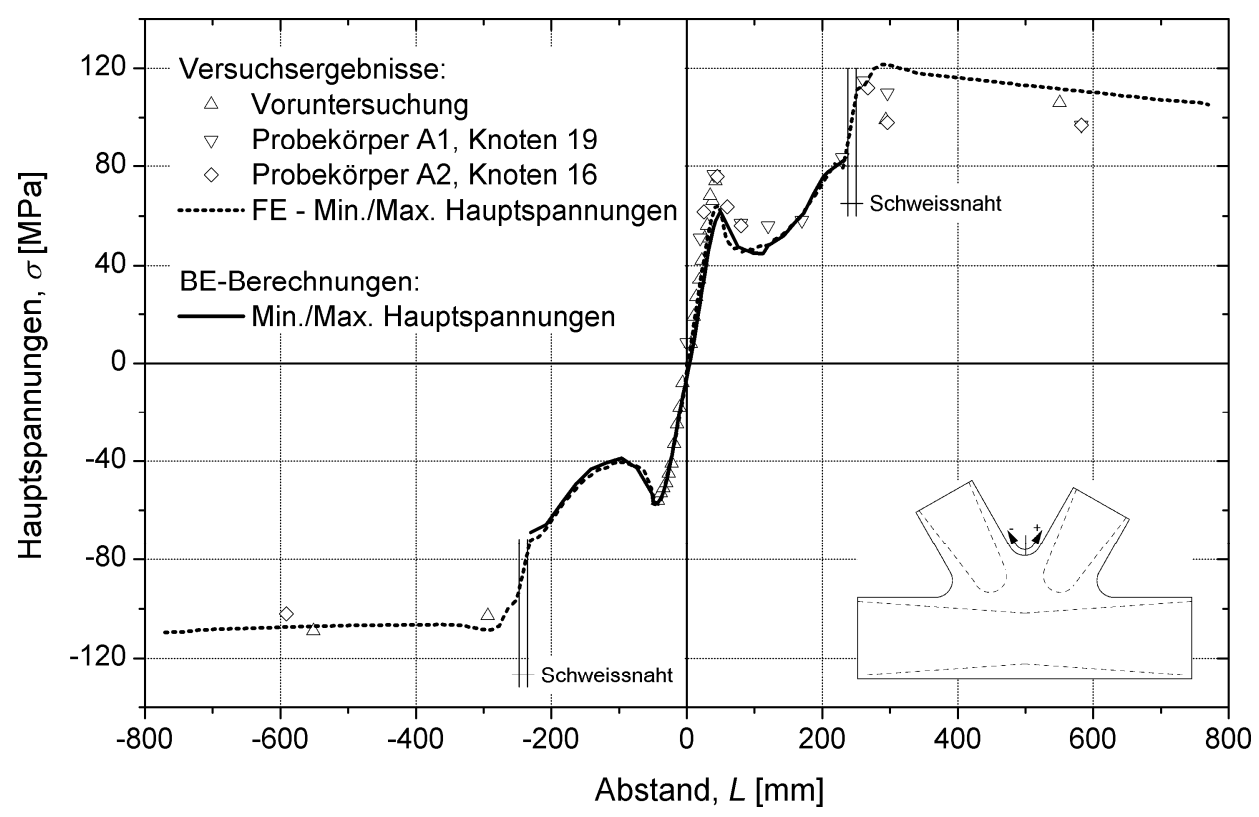

Fig. 4.12: Hauptspannungsverlauf zwischen den Diagonalstutzen, Messungen, FE- und BEBerechnungen

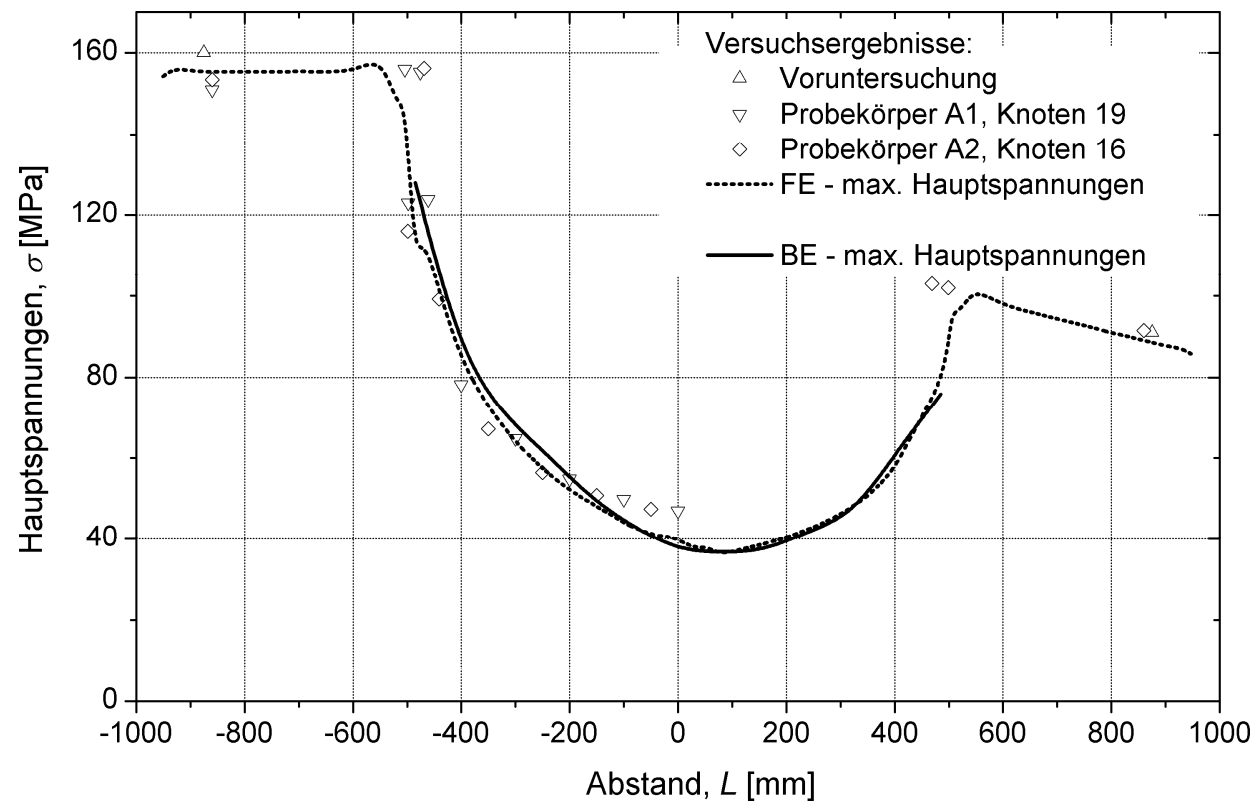

Fig. 4.13: Hauptspannungsverlauf auf der Unterseite des Untergurtstutzen, Messungen, FE- und BEBerechnungen

\subsubsection{Rissfortschrittsberechnung}

Um die Plausibilität der Rissfortschrittssimulation mit dem BE-Modell zu überprüfen, werden die Ergebnisse mit dem beobachteten Risswachstum in den Schweissnähten verglichen. Wie bereits in Abschnitt 3.5.2 beschrieben, konnten die Risse in den Stumpfschweissnähten erst bei Wanddurchbruch visuell beobachtet werden. Erste Anzeichen für einen von der Schweissnahtwurzel ausgehenden Riss konnten nur mittels der nahe der Schweissnaht aufgebrachten Dehnmessstreifen festgestellt werden. Da in den Versuchen kein Risswachstum in den Stahlgussknoten selber auftrat, kann dort die Simulation nicht validiert werden. 
Für die Simulation des Risswachstums in der Schweissnaht werden folgende Annahmen getroffen:

- Die Rissentstehungsphase wird vernachlässigt.

- Für die Rissfortschrittsberechnungen wird die linear-elastische Bruchmechanik angewendet.

- Der Anfangsriss ist ein wurzelseitig liegender, halbkreisförmiger Oberflächenriss mit einer Risstiefe $a_{0}$ und einer Risslänge $2 c_{0}$-Riss $\left(a_{0}=c_{0 \text {-Riss }}\right)$.

- Der Übergang vom Oberflächenriss zum Durchriss wird nach [BS 7910:1999] bei einer maximalen Risstiefe $a_{\max }$ festgelegt, die $95 \%$ der Wanddicke $t$ entspricht $\left(a_{\max }=0.95 t\right)$.

- Nach dem Übergang vom Oberflächenriss zum Durchriss beträgt nach [BS 7910:1999] dessen Anfangslänge $2 c_{0, \mathrm{D}-\mathrm{Riss}}=2 \cdot 0.95 t+0.2 t$.

Das Risswachstum in der Stumpfschweissnaht wird in einem Hohlprofil mit den Abmessungen des Untergurts der Probekörper simuliert, dessen Durchmesser $244.5 \mathrm{~mm}$ und dessen Wanddicke $10 \mathrm{~mm}$ beträgt. Die genaue Konfiguration der Schweissnaht (vergleiche Fig. 3.5) wird nicht modelliert. Durch diese Vereinfachung werden die durch den exzentrischen Anschluss induzierten lokalen Sekundärbiegemomente - und somit die Spannungskonzentrationen - vernachlässigt. Die Spannungskonzentrationen werden stattdessen durch die Multiplikation der Nominalspannungen mit dem Spannungskonzentrationsfaktor (siehe Tab. 3.9) berücksichtigt.

Für die Simulation des Risswachstums im Probekörper A1 (Fig. 3.19) werden die im Untergurt gemessenen und an die Knotenstutzen extrapolierten Dehnungen aus Tab. 3.8 übernommen und in Spannungen umgerechnet. Vom Hohlprofil wird analog zum BE-Knotenmodell nur eine Längshälfte modelliert und mit den in Fig. 4.11 dargestellten Randbedingungen versehen. Die Verschiebung in $x$-Richtung auf der Symmetrieebene wird blockiert $(u=0)$, die Einspannung des Untergurtstutzens wird durch die Blockierung der Verschiebungen in $x$-, $y$-, und $z$-Richtung $(u=v=w=0)$ erreicht. Fig. $4.14 \mathrm{a})$ und b) zeigen die Risskonfigurationen für den bereits fortgeschrittenen Oberflächenriss und den Durchriss.

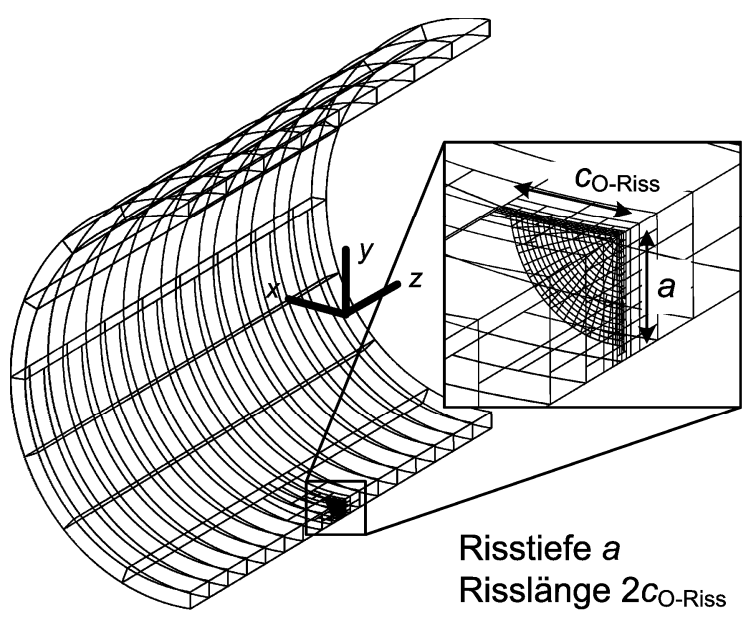

a) Oberflächenriss auf der Innenseite

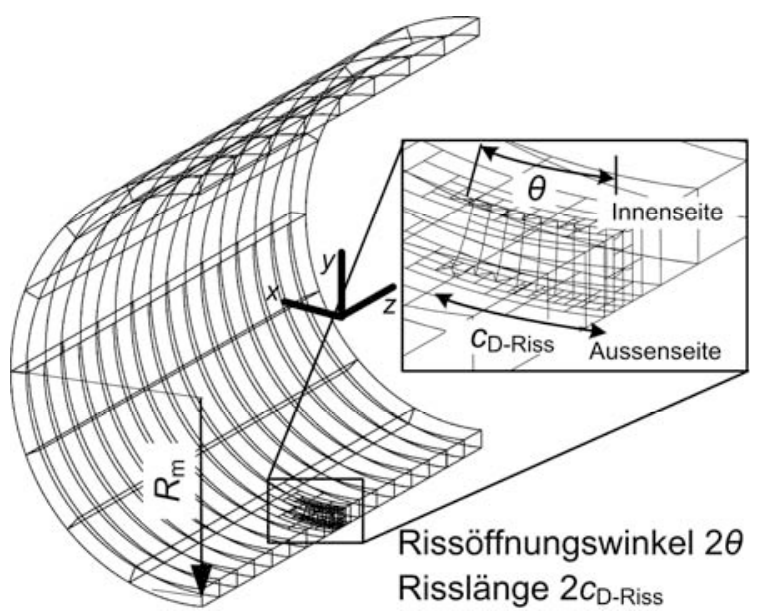

b) Durchriss

Fig. 4.14: Risskonfiguration in der Stumpfschweissnaht

Die automatische Rissfortschrittsberechnung mit BEASY besteht darin, den Initialriss inkrementell auszudehnen und für jedes Inkrement die SIF $K_{\mathrm{I}}, K_{\mathrm{II}}$ und $K_{\mathrm{III}}$ der Rissfront zu bestimmen. Für den vorliegenden Fall ist nur $K_{\mathrm{I}}$ massgebend, die beiden anderen SIF sind vernachlässigbar. Fig. 4.15 
zeigt die Spannungsintensitätsfaktoren an der Rissfront des Oberflächenrisses, wobei zwischen den Faktoren $K_{\mathrm{I}}(a)$ in der Risstiefe und $K_{\mathrm{I}}(c)$ am Rissrand unterschieden wird, da diese für eine bestimmte Rissfront unterschiedliche Werte annehmen. Die Werte $K_{\mathrm{I}}(a)$ in der Risstiefe liegen jeweils eindeutig unter denen am Rissrand. Erst bei der Annährung der Risstiefe an die Wanddicke $(t=10 \mathrm{~mm})$ steigt der SIF $K_{\mathrm{I}}(a)$ auf den Wert $K_{\mathrm{I}}(c)$ an. Es stellt sich also eine Risskonfiguration ein, bei der die halbe Risslänge $c$ grösser ist als die Risstiefe $a$ (Fig. 4.16).

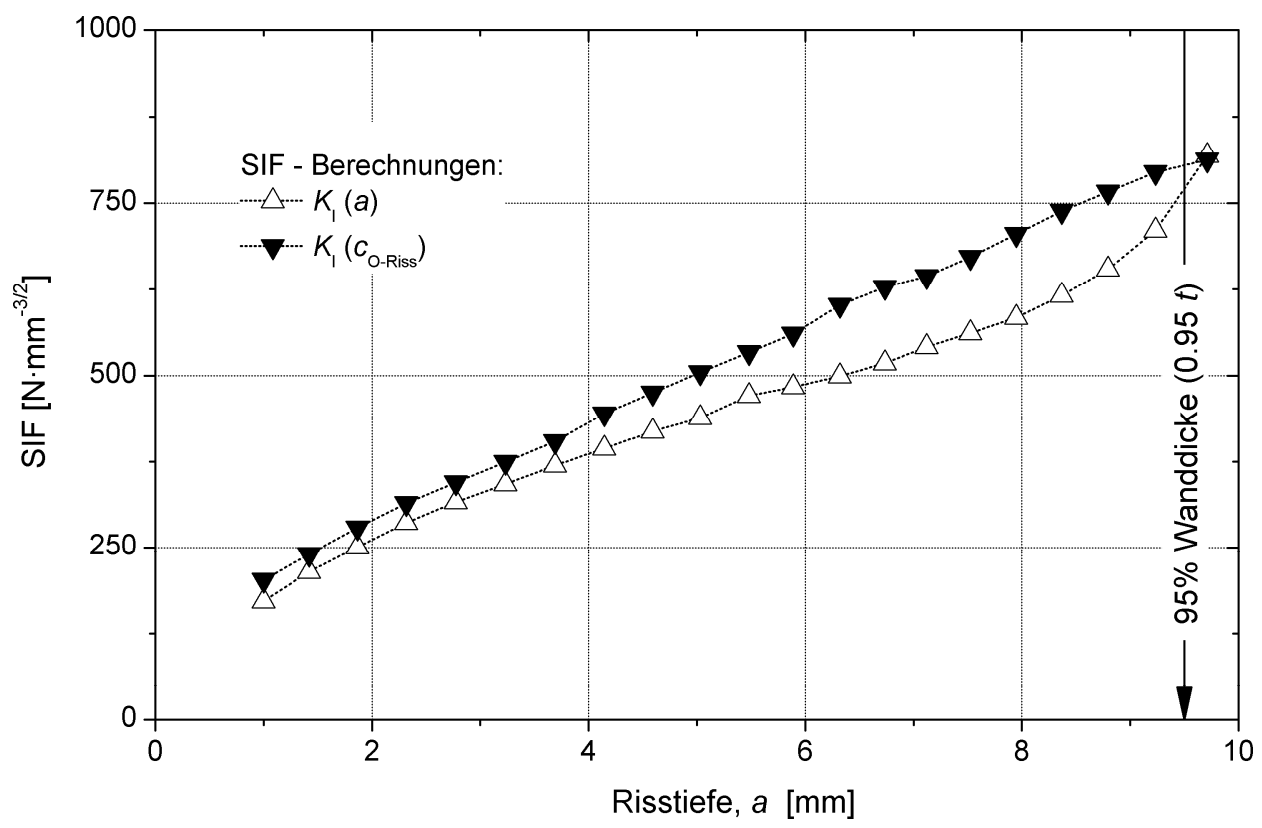

Fig. 4.15: SIF $K_{\mathrm{I}}$ für den Oberflächenriss nach BE-Berechnungen

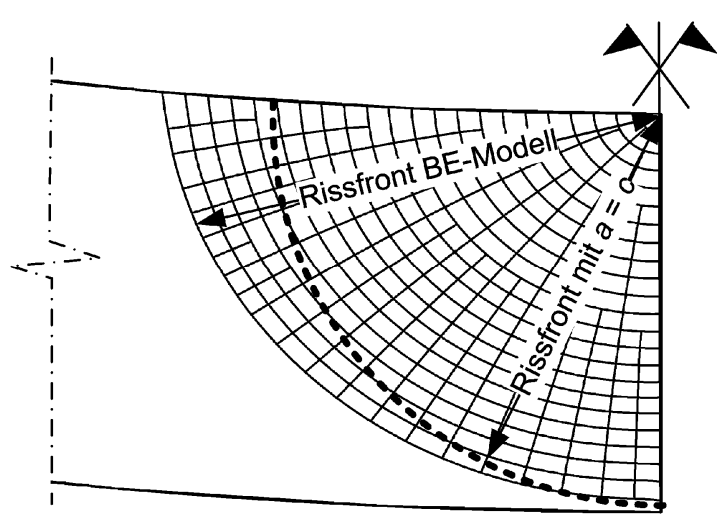

Fig. 4.16: Rissfront des Oberflächenrisses 


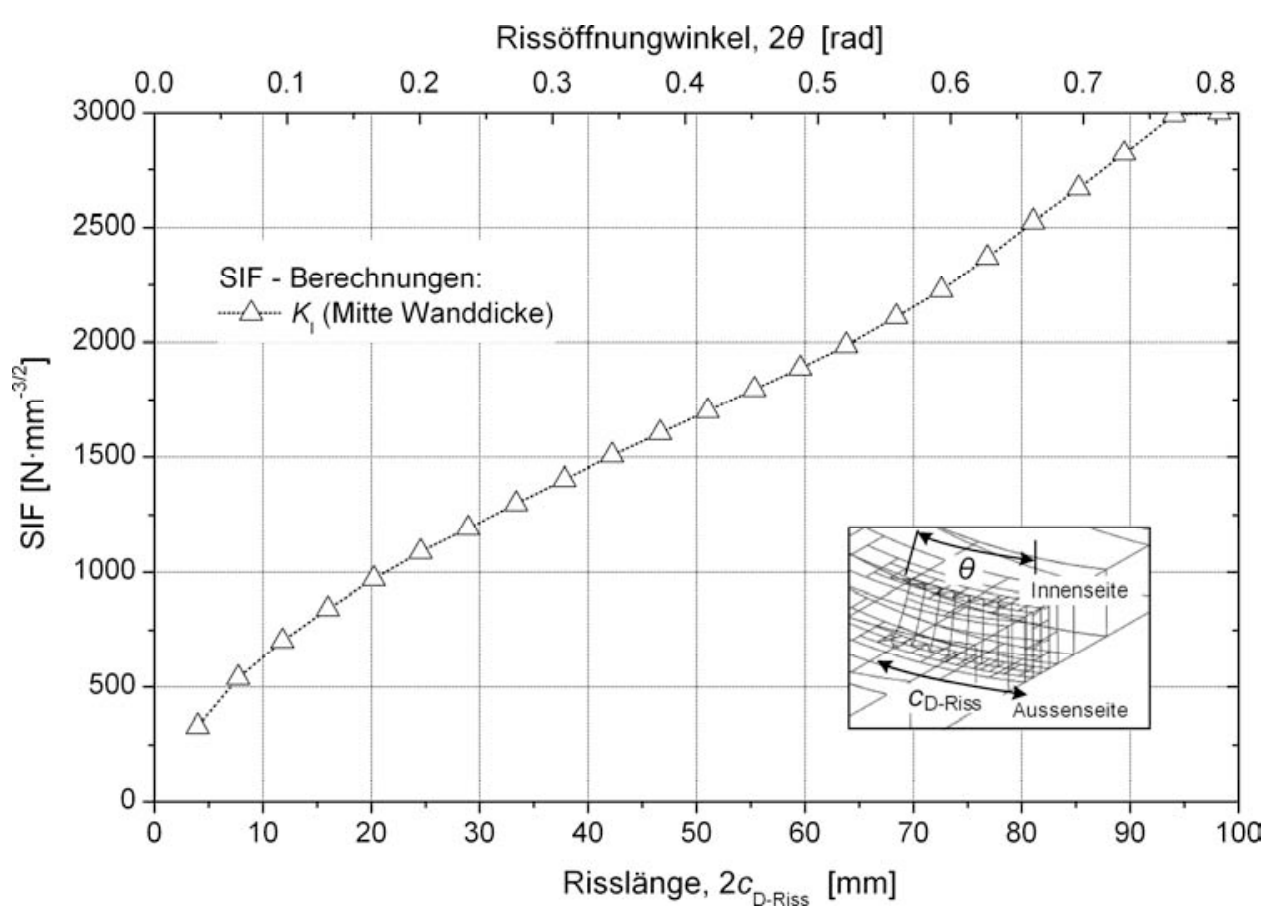

Fig. 4.17: SIF $K_{\mathrm{I}}$ für den Durchriss nach BE-Berechnungen

Fig. 4.17 illustriert den aus den BE-Berechnungen resultierenden SIF $K_{\mathrm{I}}$ auf der Rissfrontmitte für den Durchriss. Die SIF an der Rissfront sind auf der Innenseite etwas höher und auf der Aussenseite etwas niedriger als in der Rissfrontmitte. Für die Rissfortschrittsberechnungen wurden daher die mittleren Werte in der Rissfrontmitte benutzt.

Für das Wachstum des Oberflächenrisses wird die Paris-Erdogan Gleichung nach [Paris 1960] benutzt:

$$
\frac{\mathrm{d} a}{\mathrm{~d} N}=C \cdot \Delta K(a)^{m}
$$

a Risstiefe im tiefsten Punkt des Risses

$N \quad$ Anzahl Lastwechsel

$\Delta K(a) \quad$ Differenz des Spannungsintensitätsfaktors bei Risstiefe $a$

$C, m \quad$ Rissfortschrittskonstanten mit $C=2 \cdot 10^{-13}(\mathrm{~mm} /$ Lastwechsel $) \cdot\left(\mathrm{N} \cdot \mathrm{mm}^{-3 / 2}\right)^{-m}, m=3$

Das Wachstum des Durchrisses wird analog mit $\mathrm{d} \theta / \mathrm{d} N$ angesetzt, wobei über den halben Rissöffnungswinkel integriert wird:

$$
\frac{\mathrm{d} \theta}{\mathrm{d} N}=\frac{C \cdot \Delta K(2 \theta)^{m}}{R_{\mathrm{m}}}
$$

$\theta \quad$ halber Rissöffnungswinkel des Durchrisses (Fig. $4.14 \mathrm{~b}$ )

$\Delta K(2 \theta) \quad$ Differenz des Spannungsintensitätsfaktors auf der Rissfrontmitte bei Rissöffnungswinkel $2 \theta$

$R_{\mathrm{m}} \quad$ mittlerer Radius (Fig. $4.14 \mathrm{~b}$ ))

Wird das Wachstum des Oberflächenrisses nach den oben erwähnten Annahmen mit demjenigen des Durchrisses kombiniert, kann das gesamte Risswachstum in der Schweissnaht berechnet werden: 


$$
N=\int_{a_{0}}^{a_{\max }} \frac{\mathrm{d} a}{C \cdot \Delta K(a)^{m}}+\int_{\theta_{0}}^{\theta_{\max }} \frac{R \cdot \mathrm{d} \theta}{C \cdot \Delta K(2 \theta)^{m}}
$$

$$
\begin{array}{ll}
a_{0} & \begin{array}{l}
\text { Anfangsfehlergrösse } \\
a_{\max }
\end{array} \\
\theta_{0} & \begin{array}{l}
\text { maximale Risstiefe vor dem Übergang vom Oberflächenriss zum Durchriss; } \\
a_{\max }=0.95 t
\end{array} \\
& \text { halber Rissöffnungswinkel nach dem Übergang vom Oberflächenriss zum Durchriss } \\
& \text { bei einer Anfangsrisslänge des Durchrisses von } 2 c_{0, \mathrm{D}-\text {-Riss }}=2 \cdot 0.95 t+0.2 t \\
\theta_{\max } & \text { maximaler halber Rissöffnungswinkel }
\end{array}
$$

Da die BE-Berechnungen nur pro Inkrement des Risswachstums die SIF liefert, muss das Integral der Gleichung (4.5) diskret berechnet werden. Die Resultate werden in Fig. 4.18 dargestellt, wobei auf der Ordinate nicht wie üblich die Risstiefe, sondern die Risslänge $2 c$ aufgetragen wird, um den Oberflächenriss mit dem Durchriss kombinieren zu können.

Die Berechnungen haben gezeigt, dass das berechnete Risswachstum mit einer Anfangsfehlergrösse $a_{0}$ von ungefähr $4.5 \mathrm{~mm}$ am nahesten an den Messdaten liegt. Für einen Schweissfehler einer nicht durchgeschweissten Schweissnaht ist der Wert von $4.5 \mathrm{~mm}$ plausibel. Die Form der Risswachstumskurven bestätigt den in den Versuchen beobachteten steilen Anstieg des Wachstums, sobald der Oberflächenriss in den Durchriss übergangen ist. Bei einer Länge des Durchrisses von ungefähr $100 \mathrm{~mm}$ wurden die BE-Berechnungen eingestellt, da die Kurvenform bereits klar zu erkennen ist.

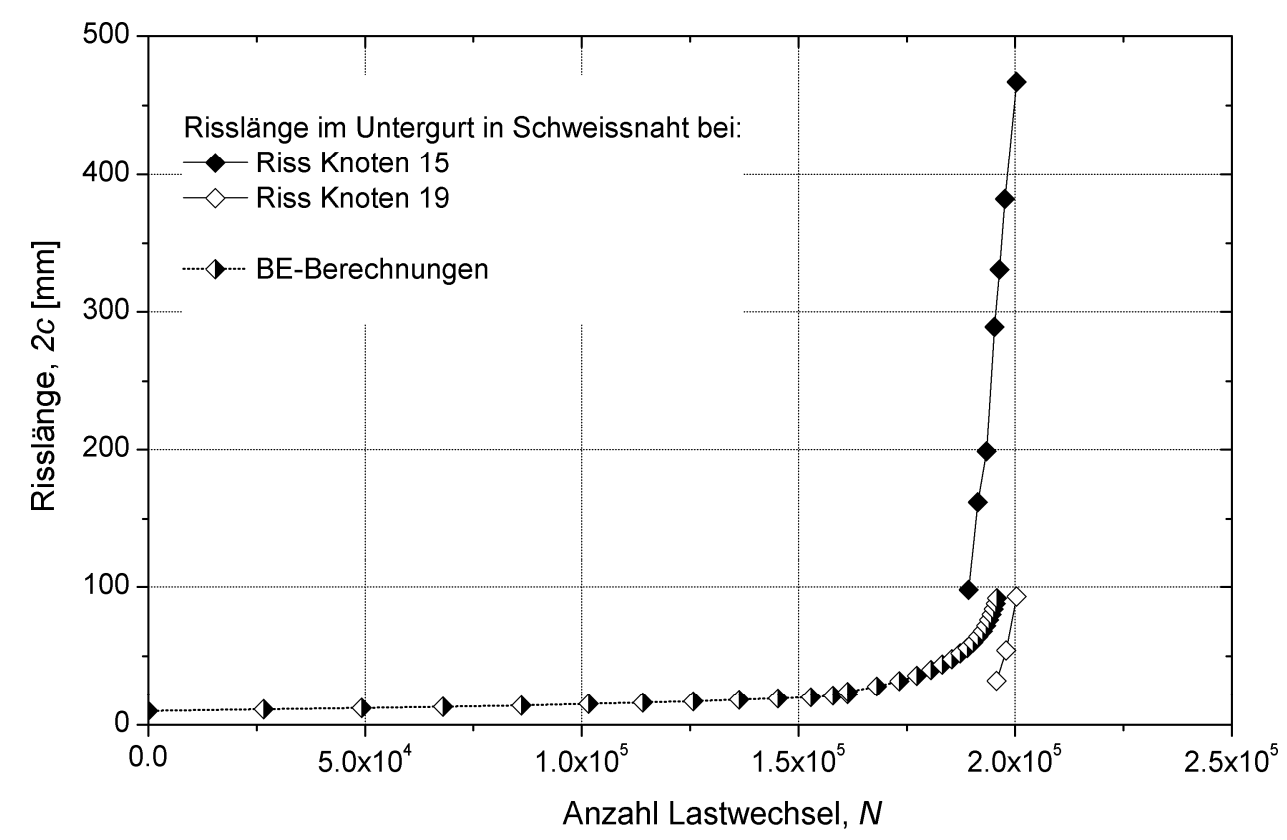

Fig. 4.18: Risswachstum: Vergleich von BE-Berechnungen mit den Messdaten für den Probekörper A1

\subsection{FOLGERUNGEN}

Um die maximal zulässigen Anfangsgrössen von Gussfehlern in Abhängigkeit von der erforderlichen Ermüdungsfestigkeit eines Stahlgussknotens definieren zu können, wird ein auf der Randelementmethode (Boundary Element Method, BEM) basierendes numerisches Modell des Knotens erstellt. So können Gussfehler im Stahlgussknoten eingefügt und ihr Wachstum simuliert werden. 
Die Anwendbarkeit des BE-Modells wird einerseits durch den Spannungsverlaufs im Stahlgussknoten und andererseits durch Nachrechnung des in den Versuchen beobachteten Risswachstums in den Schweissnähten überprüft. Auf Grund fehlender Versuchsergebnisse zum Risswachstumsverhalten von Stahlgussfehlern kann die Simulation des Risswachstums im Knoten selber nicht überprüft werden. Zur Überprüfung des Spannungsverlaufs im BE-Modell werden Berechnungen mit der Methode der finiten Elemente (Finite Element Method, FEM) sowie der in Versuchen gemessene Dehnungs- bzw. Spannungsverlauf verwendet.

Zur Modellierung mit finiten Elementen können folgende Aussagen gemacht werden:

- Der Spannungsverlauf im Stahlgussknoten kann mit dem im Rohrfachwerkträger eingebunden FE-Knotenmodell gut simuliert werden. Die Abweichungen zwischen Messdaten und Rechenergebnissen liegen grösstenteils unter $10 \%$.

- Eine kleine Abweichung der Knotengeometrie, zum Beispiel eine Verdrehung der Innenhohlräume der Diagonalstutzen um $3^{\circ}$, führt zu ungefähr 30 \% höheren Spannungen im Bereich der Spannungsspitzen zwischen den Diagonalen.

- Bei den Schweissnähten wurde der Wanddickensprung zwischen Hohlprofil und Knotenstutzen linear über eine Länge von $10 \mathrm{~mm}$ verlaufend realisiert, die genaue Geometrie mit dem exzentrischen Anschluss wurde nicht berücksichtigt. Aus diesem Grund sind die berechneten Spannungen im Schweissnahtbereich nicht repräsentativ.

Zur Modellierung mit Randelementen können folgende Aussagen gemacht werden:

- Die Ergebnisse der Spannungsberechnungen mit dem BE-Knotenmodell und den gewählten Lagerungsbedingungen stimmen gut mit den Messdaten und den FE-Berechnungen überein.

- Das beobachtete Risswachstum in den Schweissnähten eines Probekörpers kann plausibel nachgerechnet werden. Dazu wird das Risswachstum eines Oberflächenrisses mit dem eines Durchrisses kombiniert. 


\section{QUALITÄTSANFORDERUNGEN AM BEISPIEL EINER FACHWERKBRÜCKE}

\subsection{VORGEHEN}

Die Qualität von Stahlgussknoten ist in der gängigen Norm [DIN 1690-2:1985] in fünf Gütestufen eingeteilt. Für jede Gütestufe sind im Materialgefüge und an der Oberfläche zulässige Gussfehler definiert, die sich nach Lage und Grösse unterscheiden. Mit der Wahl einer hohen Gütestufe kann die Ermüdungsbemessung nicht abgedeckt werden, da zwischen Gütestufe und Ermüdungsfestigkeit keine anerkannte Relation gegeben ist. Aufgabe ist es daher nun, in Abhängigkeit von der erforderlichen Ermüdungsfestigkeit eines Stahlgussknotens die maximal zulässigen Anfangsgrössen von Gussfehlern zu definieren. Das allgemeine Vorgehen wird am Beispiel einer Modellfachwerkbrücke erklärt und in Fig. 5.1 schematisch dargestellt.

Anhand von Angaben bereits bestehender Fachwerkbrücken mit Stahlgussknoten wird zunächst eine für die Kombination von Hohlprofilen und Stahlgussknoten typische Fachwerkbrücke entworfen. Für die weiteren Untersuchungen werden zwei unterschiedlich beanspruchte Stahlgussknoten in Feldmitte und nahe dem Auflager gewählt, da unter Verkehrslast (statische Belastung) beim ersten Knoten die Untergurtstutzen und beim zweiten die Diagonalstutzen stark auf Zug belastet sind.

Die zur Rückrechnung auf die maximal zulässige Anfangsfehlergrösse benötigte Differenz des Spannungsintensitätsfaktors $\Delta K_{i}$ für Stahlgussfehler, die sich an verschiedenen Stellen $i$ im Knoten befinden, werden die SIF und die Rissgeometrie mittels der automatischen Rissfortschrittsberechnung des Programms BEASY unter der Ermüdungslast nach [SIA 261] ermittelt. Dabei wird ein Ersatzfehler eingeführt, der das Ermüdungsverhalten der verschiedenen Stahlgussfehler sicher abdeckt. Eine mögliche Vereinfachung dieses Vorgehens besteht darin, den Gussfehler mit einem Oberflächen- oder Innenriss in einem unendlichen Halbraum anzunähern und die Rückrechnung anhand eines mit einem konstanten Korrekturfaktor formulierten Spannungsintensitätsfaktors durchzuführen. Im Kapitel 6 wird auf diese Vereinfachung näher eingegangen.

Die massgebende Fehlergrösse $a_{\text {mass }, i}$ an der Stelle $i$ für die Rückrechnung auf die maximal zulässige Fehlergrösse $a_{0, i}$ wird wie folgt festgelegt: Ein Wanddurchbruch des Risses wird als nicht tolerierbar eingestuft. Nach Abzug einer Marge von $10 \%$ folgt daraus das erste Kriterium, nämlich dass $a_{\text {mass }, i}$ kleiner sein muss als $90 \%$ der Wanddicke $w$. Das zweite Kriterium folgt direkt aus dem Sprödbruchnachweis (siehe folgender Abschnitt), indem $a_{\text {mass }, i}$ kleiner sein muss als die kritische Risstiefe für Sprödbruch $a_{\text {krit }}$ Die massgebende Fehlergrösse ist also $a_{\text {mass }, i}=\min \left(0.9 w ; a_{\text {krit }}\right)$. Um die kritische Fehlergrösse für Sprödbruch im Failure Assessment Diagramm (FAD) bestimmen zu können, sind die SIF in der Risstiefe $K_{\mathrm{ULS}}^{\mathrm{a}}(a)$ und am Rissrand $K_{\mathrm{ULS}}^{\mathrm{c}}(a)$ für die massgebende Rissgeometrie unter statischer Belastung (ULS) nach [SIA 261] notwendig, die mittels des Programms BEASY ermittelt werden.

Die von der massgebende Fehlergrösse ausgehende Rückrechnung auf die maximal zulässige Anfangsfehlergrösse $a_{0, i}$ erfolgt mit der Paris-Erdogan Gleichung nach [Paris 1960] und berücksichtigt eine Lebensdauer von $2 \cdot 10^{6}$ Lastwechseln, was nach der Norm [SIA 261] einer Nutzungsdauer von 70 Jahren entspricht. 


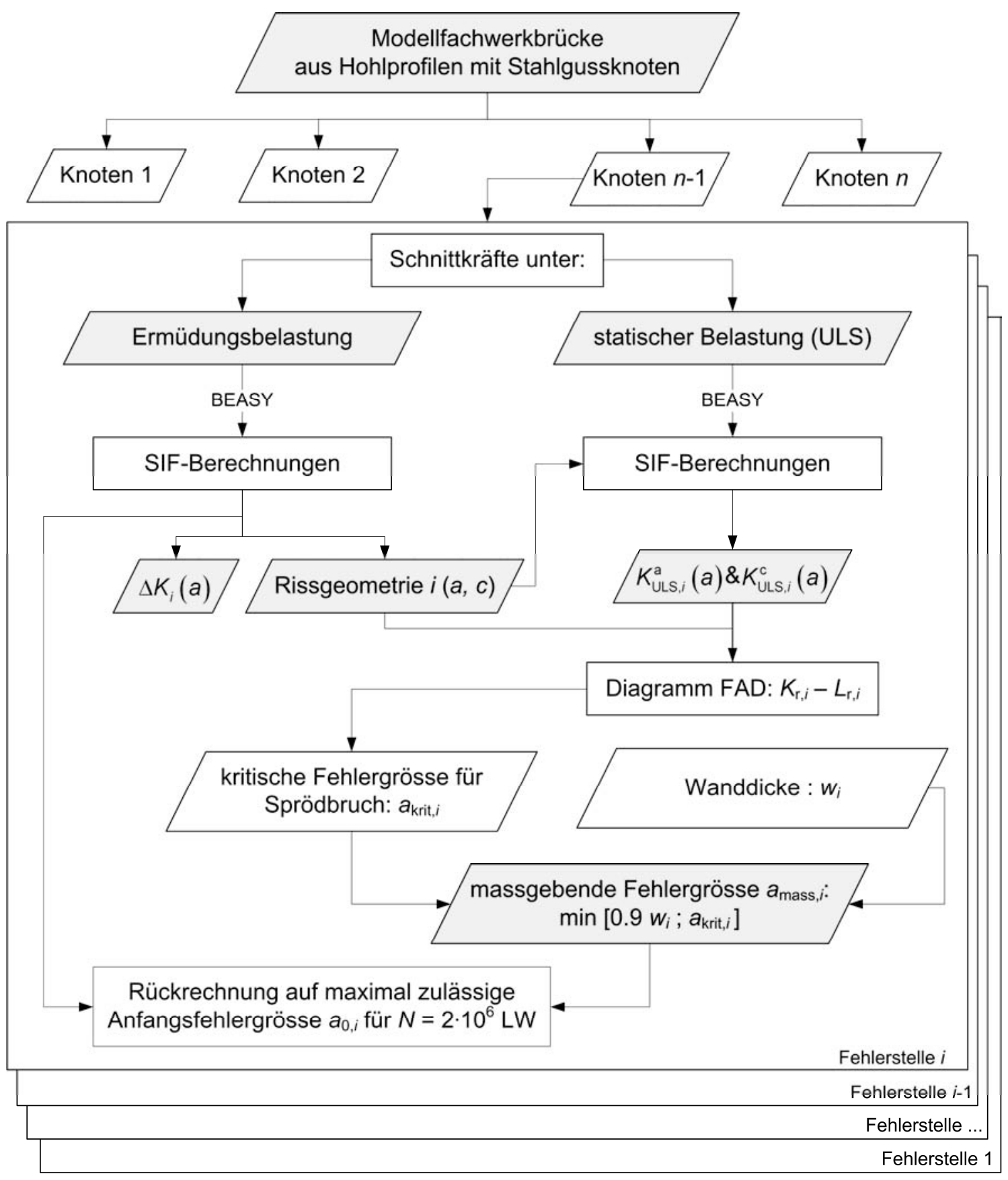

Fig. 5.1: Schematische Darstellung des Vorgehens

\subsection{MODELLFACHWERKBRÜCKE}

\subsubsection{Beschreibung}

In Abschnitt 2.1.2 findet sich eine Übersicht bestehender Fachwerkbrücken mit Stahlgussknoten. Zusätzlich sollen hier für einen besseren Überblick über Fachwerkbrücken mit Hohlprofilen auch einige Brücken mit direkt geschweissten Knoten [Dauner 1998] [Dauner et al. 1998] [Stucki et al. 2000] [Schumacher et al. 2003] herangezogen werden. Die Angaben zu den Brücken wurden bereits grösstenteils in [Kuhlmann et al. 2002] zusammengefasst, in Tab. 5.1 sind sie nochmals dargestellt. 


\begin{tabular}{lccccccc}
\hline Name & Dättwil & Lully & $\begin{array}{c}\text { Aar- } \\
\text { wangen }\end{array}$ & $\begin{array}{c}\text { Nesen- } \\
\text { bachtal }\end{array}$ & $\begin{array}{c}\text { Korntal- } \\
\text { Mün- } \\
\text { chingen }\end{array}$ & $\begin{array}{c}\text { Ooster- } \\
\text { beek }\end{array}$ & $\begin{array}{c}\text { Modell- } \\
\text { brücke }\end{array}$ \\
\hline Knotenausbildung & geschweisst & geschweisst & geschweisst & Guss & Guss & Guss & Guss \\
\hline Standort & CH & CH & CH & D & D & NL & - \\
\hline Baujahr & 2001 & 1997 & 1997 & 1999 & 2002 & 2002 & - \\
\hline Nutzung & Autobahn & Autobahn & Hauptstrasse & Hauptstrasse & Hauptstrasse & Eisenbahn & Hauptstrasse \\
\hline Fahrbahnplattenbreite $[\mathrm{m}]$ & 13.25 & 13.25 & 8.30 & 11.5 & 12 & 12.2 & 11 \\
\hline Fachwerkhöhe $[\mathrm{m}]$ & 3.3 & 2.9 & 1.77 & 2.28 & 2.6 & 4.24 & 2.38 \\
\hline Spannweite $[\mathrm{m}]$ & $38 / 26$ & 43 & 48 & $25 / 50 / 36$ & $32 / 41$ & 57.7 & 40 \\
\hline Schlankheit & $1 / 8-1 / 12$ & $1 / 15$ & $1 / 24$ & $1 / 11-1 / 22$ & $1 / 12-16$ & $1 / 14$ & $1 / 16.8$ \\
\hline Knotenform & $\mathrm{KK}$ & $\mathrm{KK}$ & $\mathrm{K}$ & $\mathrm{KK}$ & $\mathrm{KK}$ & $\mathrm{K}$ & $\mathrm{K}$ \\
\hline Längswinkel $\theta\left[^{\circ}\right]$ & 60 & 60 & 45 & 46 & 60 & 54 & 55 \\
\hline Querwinkel $\phi\left[^{\circ}\right]$ & 69 & 69 & - & 102 & 90 & - & - \\
\hline Diagonalen $d / t$ & $267 / 11-25$ & $267 / 11-25$ & $194 / 20-28$ & $194 / 20-28$ & $267 / 28-45$ & $406 / 30$ & $273 / 10-25$ \\
\hline Untergurt $D / T$ & $508 / 50$ & $508 / 25-50$ & $406 / 36-50$ & $324 / 16-80$ & $457 / 45-65$ & $610 / 50-80$ & $457 / 12.5-36$ \\
\hline$\beta=d / D$ & 0.53 & 0.53 & 0.48 & 0.60 & 0.58 & 0.67 & 0.60 \\
\hline
\end{tabular}

Tab. 5.1 : Übersicht Fachwerkbrücken aus Hohlprofilen mit Kreisquerschnitt

Obwohl diese Brücken sowohl in der Grösse als auch in der Gestaltung wesentlich von einander abweichen, wurde versucht, anhand dieser bestehenden Brücken eine „typische“ Rohrfachwerkbrücke mit Stahlgussknoten zu definieren, für welche Untersuchungen zu den maximal zulässigen Gussfehlergrössen durchgeführt werden sollen. Diese Modellfachwerkbrücke wurde mit geringfügigen Anpassungen auch für Untersuchungen an nachbehandelten, direkt geschweissten Knoten verwendet [Walbridge 2005]. Die Kenndaten des Brückenbeispiels (Fig. 5.2) können wie folgt zusammengefasst werden und sind in Tab. 5.1 den bestehenden Brücken gegenübergestellt:

- Hauptverkehrsbrücke über 3 Felder mit einer Spannweite von $40 \mathrm{~m}$.

- Offener Querschnitt mit zwei parallelen, ebenen Fachwerkträgern aus Hohlprofilen mit Kreisquerschnitt und einer $11 \mathrm{~m}$ breiten Fahrbahnplatte für zwei Fahrbahnspuren.

- Die Fahrbahnplatte misst $300 \mathrm{~mm}$ über den Fachwerkträgern und nimmt an den Enden auf $250 \mathrm{~mm}$ ab, woraus sich eine mittlere Dicke von $280 \mathrm{~mm}$ ergibt.

- Die Stahlgussknoten sind als ebene K-Knoten mit einem Winkel der Diagonalen von $\theta=55^{\circ}$ ausgebildet.

- Für den festgelegten Winkel $\theta$ und 13 Knoten im Untergurt (12 Unterteilungen) pro Feld ergeben sich eine Fachwerkträgerhöhe von $2380 \mathrm{~mm}$ und somit eine Schlankheit (definiert durch das Verhältnis der Trägerhöhe zur Spannweite) von $1 / 16.8$.

- Die Aussendurchmesser des Untergurts und der Diagonalen wurden mit 457 mm bzw. 273 mm festgelegt. Somit liegt das Verhältnis des Aussendurchmessers der Diagonalen $d$ zum Aussendurchmesser des Untergurts $D$ bei $\beta=d / D=0.60$. 

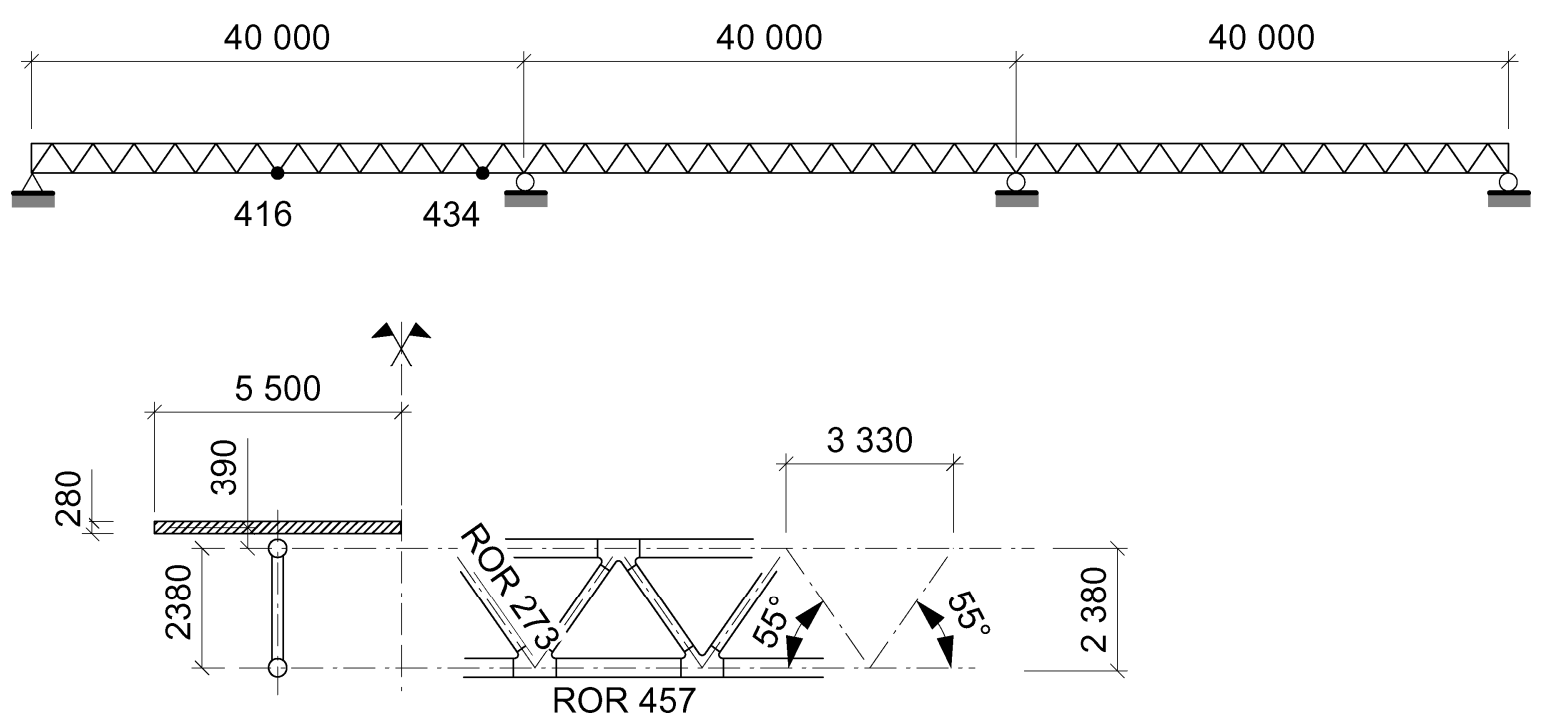

Fig. 5.2: Modellfachwerkbrücke, statisches Modell

\subsubsection{Form der Stahlgussknoten für die Modellfachwerkbrücke}

Der Längsschnitt der gewählten Knotenform für die Modellfachwerkbrücke wird in Fig. 5.3 schematisch dargestellt. Der Wahl der Geometrie liegen folgende Annahmen und giesstechnischen Regeln zu Grunde:

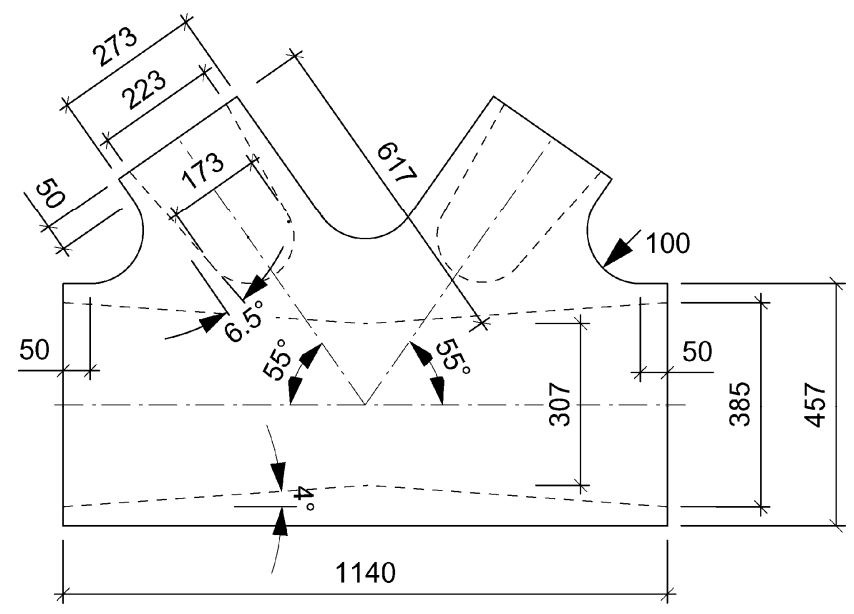

Fig. 5.3: Knotenform für die Modellfachwerkbrücke

- Für die gesamte Modellfachwerkbrücke wird eine einzige Knotenform festgelegt.

- Die Wanddicken an den Enden der Stutzen entsprechen der maximalen Wanddicke im Untergurt $(T=36 \mathrm{~mm})$ respektive in den Diagonalen $(t=25 \mathrm{~mm})$.

- Die Fugenvorbereitung an den Stutzenenden mit der je nach Brückenposition entsprechend angepassten Wanddicke wird nicht in die Knotenform integriert. Somit kann bei der Herstellung der Gussknoten für alle Knoten dasselbe Modell ${ }^{6}$ benutzt werden und nur die Stutzenenden müssen mit speziell angefertigten Ansätzen ergänzt werden.

\footnotetext{
${ }^{6}$ Für die Herstellung von Gussteilen wird ein Modell (meist aus Holz) des Gussteils hergestellt, mit welchem die Negativform des Gussteils in den Formstoff gepresst wird.
} 
- Die Stutzenlängen im Untergurt und für die Diagonalen werden auf ein Minimum beschränkt. Die minimale Länge ergibt sich durch die Ausrundungen zwischen den Stutzen mit einem Radius von $100 \mathrm{~mm}$ und dem Abstand von $50 \mathrm{~mm}$ von der Ausrundung bis zu den Anschweissenden. Dieser Abstand ist notwendig, damit die Schweissnähte nicht in Bereiche von Spannungskonzentrationen fallen (vergleiche Fig. 4.5).

- Die Bildung von Lunkern (siehe Abschnitt 2.2.1) wird durch eine Wanddickenzunahme mit einem Mindestwinkel von $4^{\circ}$ verhindert.

\subsubsection{Modellierung der Stahlgussknoten im Stabmodell der Brücke}

Im Stabmodell der Brücke werden auch die Stahlgussknoten durch Stäbe modelliert. Aus der gewählten Knotenform (Fig. 5.3) werden für den Untergurtstutzen und die Diagonalstutzen die entsprechenden, in Fig. 5.4 dargestellten, Ersatzstäbe herausgelesen, wobei die variablen Wanddicken beibehalten werden. Die Ersatzstäbe des Knotens sind im Stabmodell der Brücke eingespannt.

Die Gültigkeit dieser vereinfachten Modellierung wurde an den Probekörpern überprüft. Die Stahlgussknoten der Versuchsserie wurden durch Ersatzstäbe im Stabmodell des Probekörpers modelliert. Der Vergleich der Nominalspannungen in den Fachwerkstäben an den Stellen der Dehnmessstreifen zeigt mit den Rechenwerten eine sehr gute Übereinstimmung.

Die Auswertung der Versuchsergebnisse in Abschnitt 3.5.1 hat gezeigt, dass die Verkürzung der Stutzenlängen zwei Auswirkungen hat. Einerseits verschiebt sich die Schweissnaht gegen den Achsenschnittpunkt zu, also in Richtung höherer Sekundärbiegemomente. Andererseits werden die Rundhohlprofile länger, ihre Biegesteifigkeit und damit die Sekundärbiegemomente nehmen ab. Im Fall der Modellfachwerkbrücke werden die Knotenstutzen mit minimaler Länge ausgeführt, da in den Diagonalen der erste Effekt überwiegt, dies also der ungünstigste Fall ist. Ausserdem werden durch kürzere Stutzenlängen Knotengewicht und damit Herstellungskosten eingespart. Im Untergurt heben sich die zwei Effekte weitgehend auf.

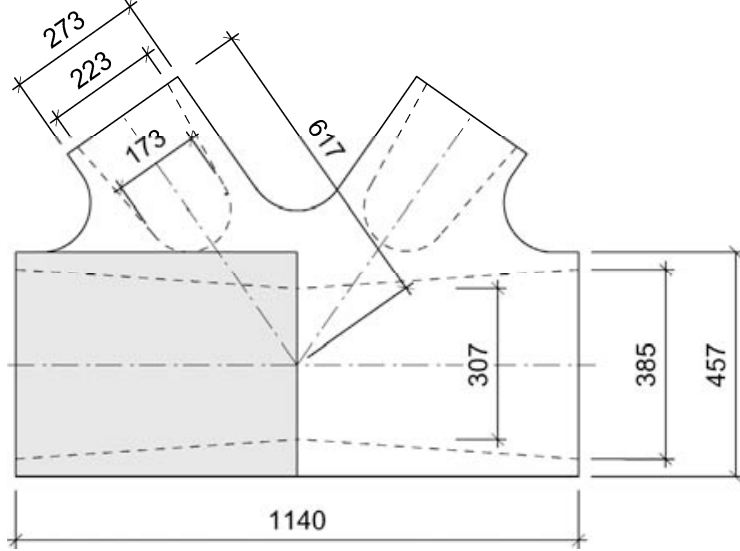

a) Untergurt

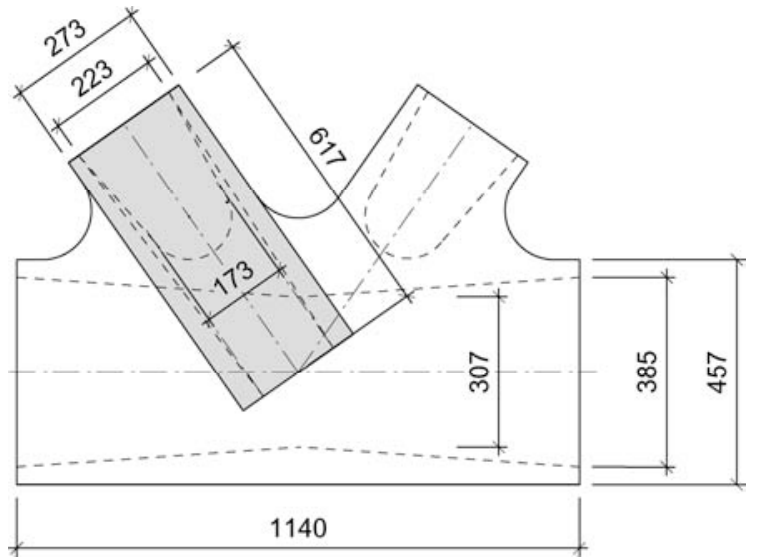

b) Diagonale

Fig. 5.4: Modellierung der Stahlgussknoten im Stabmodell der Brücke 


\subsubsection{Statische Bemessung}

Die Brücke wird hinsichtlich der Tragsicherheit und der Gebrauchstauglichkeit nach den aktuellen Schweizer Normen [SIA 261] und [SIA 263] bemessen. Der Einfachheit halber und um des Brückenmodell so allgemein wie möglich zu gestalten, werden nur das Eigengewicht und die Verkehrslasten berücksichtigt; Schnee- und Windlasten, Erdbeben und Temperatureinflüsse werden nicht betrachtet.

\section{Berechnungsannahmen}

- In Feldmitte beträgt der Bewehrungsgehalt der Fahrbahnplatte $1.0 \%$, über den Auflagern $1.5 \%$.

- Über den Auflagern ist der zugbeanspruchte Stahlbeton nicht tragend, die Zugbeanspruchung wird ausschliesslich durch die Bewehrung aufgenommen.

- Das Verhältnis des Elastizitätsmoduls des Stahls $E_{\text {Stahl }} z u$ demjenigen des Stahlbetons $E_{\text {Beton }}$ wird mit $E_{\text {stahl }} / E_{\text {Beton }}=10$ angenommen.

- Die Quereinflusslinie für die Verkehrslasten nimmt den Wert 0.9 über dem einen Längsträger und 0.1 über dem anderen an.

- Die Fahrbahnplatte wird während dem Bau durch ein Gerüst getragen und belastet somit die Längsträger während der Bauphase nicht.

\section{Ständige Einwirkungen}

Nach [SIA 260] wird für die ständigen Einwirkungen ein Lastbeiwert $\gamma_{\mathrm{F}}$ von 1.35 bei ungünstig wirkenden und 0.80 bei günstig wirkenden Lasten angenommen. Folgende ständige Einwirkungen werden berücksichtigt:

- Eigengewicht der Fachwerkträger (ohne Eigengewicht von Querstreben)

- Eigengewicht der Fahrbahnplatte, inklusive der Geländer

- 100 mm dicker Fahrbahnbelag.

\section{Veränderliche Einwirkungen}

Nach [SIA 260] wird für die Verkehrslasten ein Lastbeiwert $\gamma_{\mathrm{F}}$ von 1.50 angenommen. Es wird das Verkehrslastmodell nach [SIA 261] herangezogen. Vier Lastfälle werden für die statische Bemessung untersucht, wobei die Lasten so angesetzt werden, dass für das mittlere Feld entweder das Biegemoment oder die Querkraft in Feldmitte oder über dem Auflager am grössten ist.

\subsubsection{Ermüdungsbemessung}

Für die Ermüdungsbemessung werden zunächst nur die Stumpfschweissnähte zwischen den Hohlprofilen und den Stutzen der Stahlgussknoten betrachtet. Aus der statischen Bemessung und der Ermüdungsbemessung ergeben sich die nötigen Wanddicken der Hohlprofile, mit denen anschliessend die Knotenform definiert wird.

\section{Berechnungsannahmen}

- Für die Ermüdungslast wird das Lastmodell nach [SIA 261] herangezogen.

- Nach [SIA 260] wird für die Ermüdungslast ein Lastbeiwert $\gamma_{\mathrm{F}}$ von 1.00 angenommen.

- Der Widerstandsbeiwert der Ermüdungsfestigkeit $\gamma_{\mathrm{Mf}}$ wird nach [SIA 263] mit 1.15 angesetzt.

- Die Nutzungsdauer der Brücke beträgt nach [SIA 261] 70 Jahre.

- Über die Brücke verläuft eine Hauptverkehrsstrasse. 
- Die Stumpfschweissnähte sind in Anlehnung an die Fugenvorbereitung der Versuchsserie B mit einer Schweissbadsicherung (Fig. 3.5) ohne exzentrischen Anschluss ausgebildet.

- Die Ermüdungsfestigkeit der Stumpfschweissnähte entspricht $\Delta \sigma_{\mathrm{C}}=87 \mathrm{MPa}$ (siehe Abschnitt 3.5.2.3).

- Der Kerbfall wird nach [SIA 263] mit dem Faktor $k_{\mathrm{s}}=(25 / t)^{0.2}$ reduziert, sollte die Wanddicke $t>25 \mathrm{~mm}$ betragen.

- Für die Ermüdungsbemessung wird angenommen, dass zwischen den Längsträgern keine Interaktion besteht und die auf eine Brückenhälfte wirkende Ermüdungslast somit zu 100\% von einem Längsträger aufgenommen wird.

- Der schadensäquivalente Betriebslastfaktor $\lambda$ nach [SIA 263] besteht aus dem Produkt der Betriebslast-Teilfaktoren $\lambda_{1}, \lambda_{3}$ und $\lambda_{4}$ :

Für Brücken von Hauptverkehrsstrassen mit einer Einflusslänge von $40 \mathrm{~m}$ hat der Betriebslast-Teilfaktor $\lambda_{1}$ den Wert 1.4.

Für eine Nutzungsdauer von 70 Jahren beträgt der Betriebslast-Teilfaktor $\lambda_{3}=1.0$.

Der Betriebslast-Teilfaktor $\lambda_{4}$ berücksichtigt mehrere Fahrspuren auf einem Tragwerk. Da auf einen Längsträger nur der Verkehr einer Fahrspur einwirkt, gilt der Wert 1.0.

Der schadensäquivalente Betriebslastfaktor $\lambda$ beträgt somit 1.4.

\subsubsection{Ergebnisse aus statischer Bemessung und Ermüdungsbemessung}

Die Berechnungen wurden an einem Stabmodell mit Hilfe des Programms RSTAB [RSTAB 2003] durchgeführt. Die Modellierung der Stahlgussknoten wird in den Abschnitten 5.2.2 und 5.2.3 erklärt. Bei jeder Iteration wurde die Geometrie der Stahlgussknoten neu angepasst.

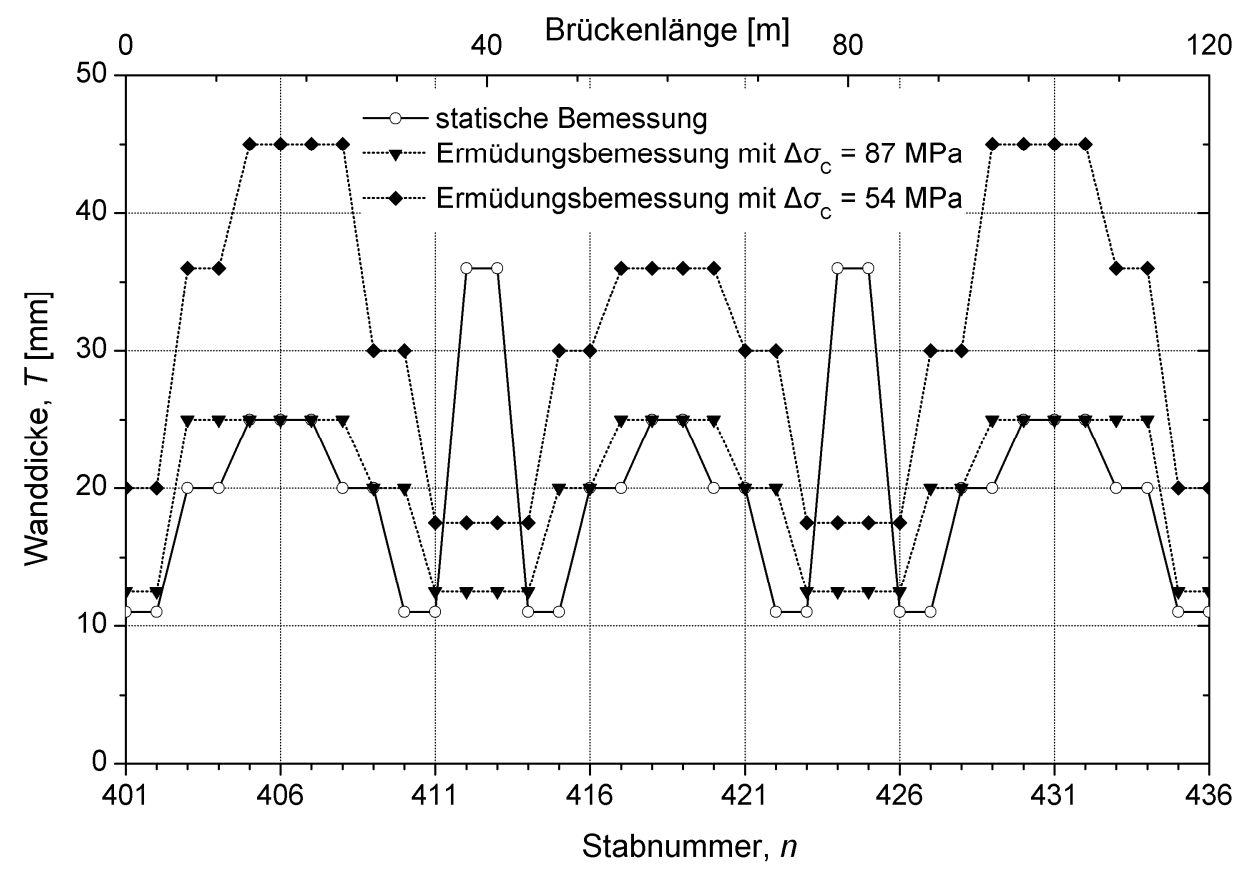

Fig. 5.5: Wanddicken für die Hohlprofile im Untergurt $(D=457 \mathrm{~mm})$

In Fig. 5.5 und Fig. 5.6 werden die Ergebnisse für den Untergurt und die Diagonalen dargestellt. Die Abszisse zeigt jeweils die Stabnummern und die Brückenlänge, die Ordinate die Wanddicke. Der Vergleich der Ergebnisse aus den statischen Berechnungen mit denjenigen der Ermüdungsbe- 
messung zeigt sowohl für den Untergurt als auch für die Diagonalen, dass bei Annahme einer Ermüdungsfestigkeit mit $\Delta \sigma_{\mathrm{C}}=87 \mathrm{MPa}$ die Wanddicken der Hohlprofile nicht wesentlich erhöht werden müssen.

Hingegen würden bei einer den Versuchsergebnissen für die Serie A (Abschnitt 3.5.2.3) entsprechenden Ermüdungsfestigkeit mit $\Delta \sigma_{\mathrm{C}}=54 \mathrm{MPa}$ die erforderlichen Wanddicken auf Grund der Ermüdungsbemessung massiv ansteigen. Für die weiteren Untersuchungen werden die Ergebnisse für $\Delta \sigma_{\mathrm{C}}=87 \mathrm{MPa}$ beibehalten. Die gewählten Wanddicken für den Untergurt und die Diagonalen ergeben sich als Maximum der Ergebnisse der statischen Bemessung und der Ermüdungsbemessung.

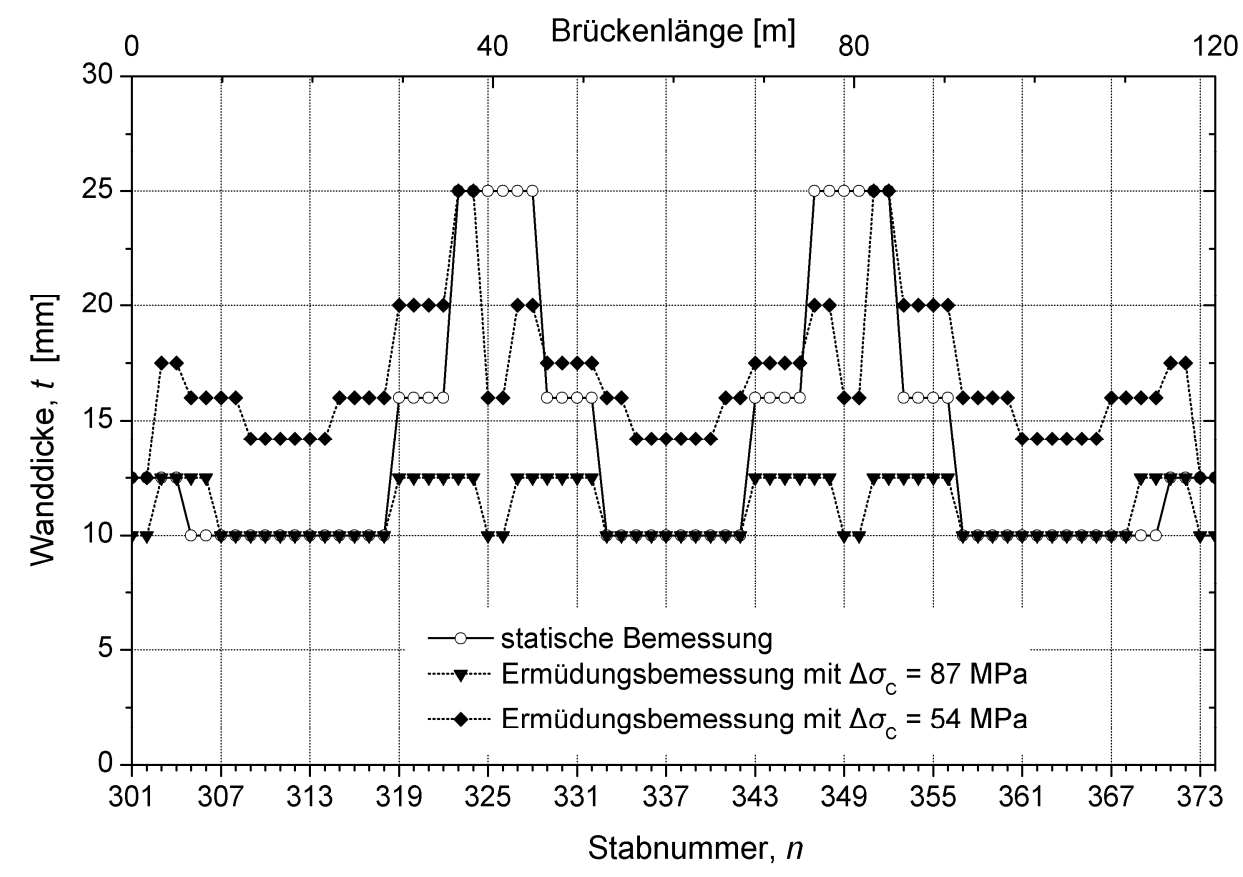

Fig. 5.6: Wanddicken für die Hohlprofile in den Diagonalen $(d=273 \mathrm{~mm})$

\subsection{BELASTUNGSZUSTÄNDE DER STAHLGUSSKNOTEN}

\subsubsection{Wahl der zu untersuchenden Knoten}

Für die Wahl der zu untersuchenden Knoten wird die Ermüdungsbelastung als Kriterium herangezogen. Die zwei zu untersuchenden Knoten sollen sich durch eine sehr hohe Zugkraft entweder im Untergurtstutzen oder in einem der Diagonalstutzen auszeichnen.

Für jeden Knoten in der Modellfachwerkbrücke werden für alle möglichen Positionen der Ermüdungslast entlang der Brücke aus dem Stabmodell die maximale Zugkraft im rechten Untergurtstutzen sowie im Diagonalstutzen und die dazugehörigen Axialkräfte in den anderen Stutzen bestimmt. Die Umhüllende der Maximalwerte der Zugkraft im rechten Untergurtstutzen ist in Fig. 5.7 dargestellt; der Maximalwert tritt im Knoten 416 auf. Im Diagonalstutzen (Fig. 5.8) tritt das Maximum im Knoten 434 auf. Wie aus den beiden Figuren ersichtlich ist, unterscheiden sich die Maximalwerte der Zugkraft im Untergurt und in der Diagonale nur geringfügig. Die Position der beiden Knoten innerhalb der Brücke ist auch aus Fig. 5.2 ersichtlich. 


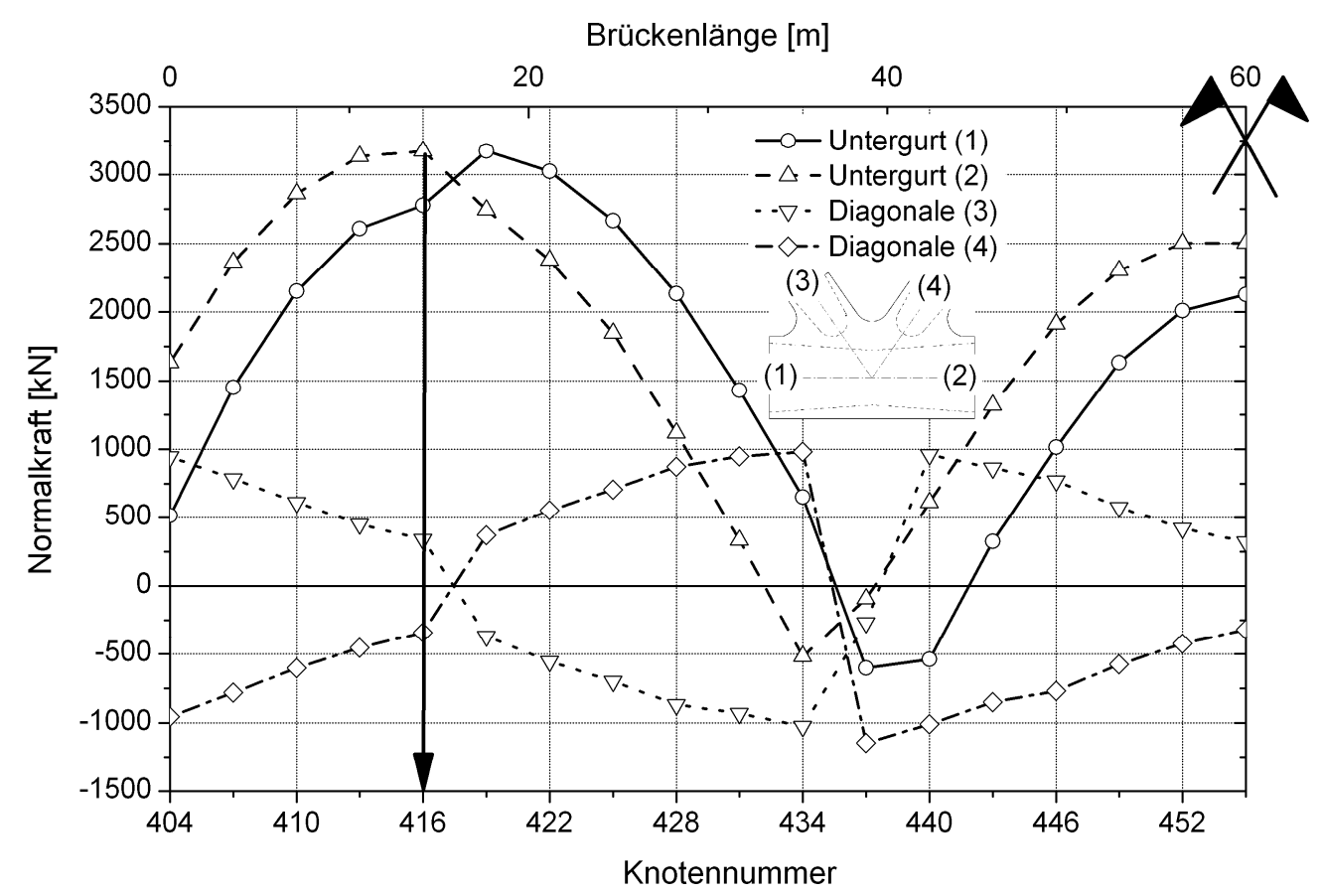

Fig. 5.7: Umhüllende der Zugkraft im rechten Untergurtstutzen

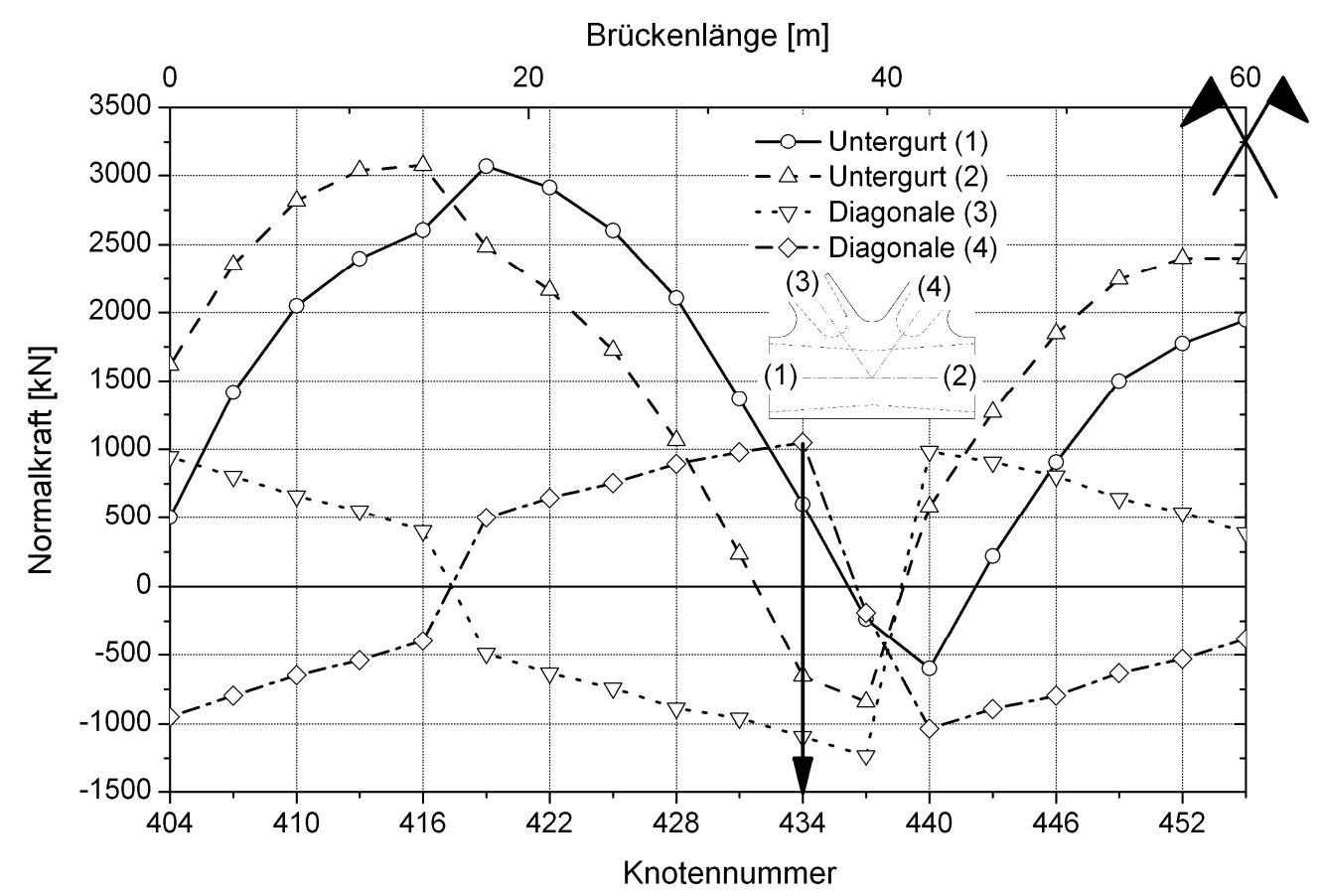

Fig. 5.8: Umhüllende der Zugkraft im Diagonalstutzen

\subsubsection{Statische Belastung (ULS)}

Für den Sprödbruchnachweis des mit einem Ermüdungsriss behafteten Stahlgussknotens bei einer Temperatur während der Nutzung von $-30^{\circ} \mathrm{C}$ wird die statische Belastung unter Verkehrslast als Leiteinwirkung sowie das Eigengewicht herangezogen. Bei dieser Bemessungssituation handelt es sich um eine konservative Wahl, da im Zusammenhang mit dem Sprödbruchnachweis bei $-30^{\circ} \mathrm{C}$ eigentlich die Bauteiltemperatur die Leiteinwirkung darstellt, der dann die Verkehrslast als Begleiteinwirkung zugeordnet ist. 
Die Schnittkräfte für die gewählten Knoten 416 und 434 aus dem Stabmodell der Modellfachwerkbrücke sind in Tab. 5.2 zusammengefasst. Die massgebende Bemessungssituation zeichnet sich für den Knoten 416 durch die hohe Zugbelastung in den Untergurtstutzen aus, für den Knoten 434 durch die hohe Zug- und Druckbelastung in den Diagonalstutzen.

\begin{tabular}{lcccccc}
\hline & & & & & & \\
Knoten 434 \\
\hline Schnittkräfte & $\mathrm{N}$ & $\mathrm{Q}-3$ & $\mathrm{M}-2$ & $\mathrm{~N}$ & $\mathrm{Q}-3$ & $\mathrm{M}-2$ \\
{$[1)$} & {$[\mathrm{kN}]$} & {$[\mathrm{kN}]$} & {$[\mathrm{kNm}]$} & {$[\mathrm{kN}]$} & {$[\mathrm{kN}]$} & {$[\mathrm{kNm}]$} \\
\hline Untergurt (1) & 7601.6 & 2.8 & 117.2 & -3960.4 & -17 & 45.1 \\
\hline Untergurt (2) & 7510.1 & -3.9 & 120.3 & -7912.8 & 250 & 0.6 \\
\hline Diagonale (3) & -67.2 & 9.9 & -4.0 & -3509.1 & 81.3 & 68.0 \\
\hline Diagonale (4) & 90.6 & 11.1 & 20.6 & 3214.3 & 33.1 & 57.5 \\
\hline
\end{tabular}

Tab. 5.2 : Schnittkräfte für die Knoten 416 und 434

Da die Querkräfte im Verhältnis zu den Axialkräften sehr klein sind, jedoch geometrie- und softwarebedingt mit hohem Aufwand ins Modell eingetragen werden müssten, werden sie vernachlässigt. Die Normalkräfte und Biegemomente werden leicht korrigiert (nicht in Tab. 5.2 dargestellt), damit das statische Gleichgewicht der Knoten erhalten bleibt.

\subsubsection{Ermüdungsbelastung}

Für die Simulation des Rissfortschritts in den Stahlgussknoten wird die Ermüdungslast nach [SIA 261] verwendet (siehe Abschnitt 5.2.5). Die infolge der Überfahrt der Ermüdungslast über die Brücke an die Stahlgussknoten angreifenden Normalkräfte variieren nicht in Phase, sondern erreichen ihre Extremwerte bei unterschiedlichen Lastpositionen. Daher ist nicht offensichtlich, welche Laststellungen für eine bestimmte Fehlerstelle im Stahlgussknoten zu den minimalen bzw. maximalen Spannungsintensitätsfaktoren (SIF) $K_{\min }$ bzw. $K_{\max }$ führen, anhand derer die für die Simulation des Rissfortschritts benötigte Differenz $\Delta K$ zu berechnen ist. In diesem Abschnitt wird daher näher auf die Ermüdungsbelastung der Stahlgussknoten eingegangen.

In Fig. 5.9 und Fig. 5.10 sind die Einflusslinien der Normalkräfte infolge Ermüdungslast und Eigengewicht für die Knoten 416 und 434 dargestellt. Für den Knoten 416 liegen an den stets durch Zugkraft beanspruchten Untergurtstutzen die Maxima bei Laststellungen in der Nähe der Knotenposition in der Brücke, während an den Diagonalstutzen die Normalkraft zwischen Druck- und Zugkraft wechselt. Im Knoten 434 hingegen sind die Untergurtstutzen fast ausschliesslich auf Druck beansprucht. In den Diagonalstutzen variieren die Normalkräfte nicht wie beim Knoten 416, sondern die Zug- bzw. Druckkraft im jeweiligen Diagonalstutzen nimmt mit der Annäherung der Ermüdungslast an den Knoten stark zu, um sich danach auf ein niedriges Niveau einzufinden. 


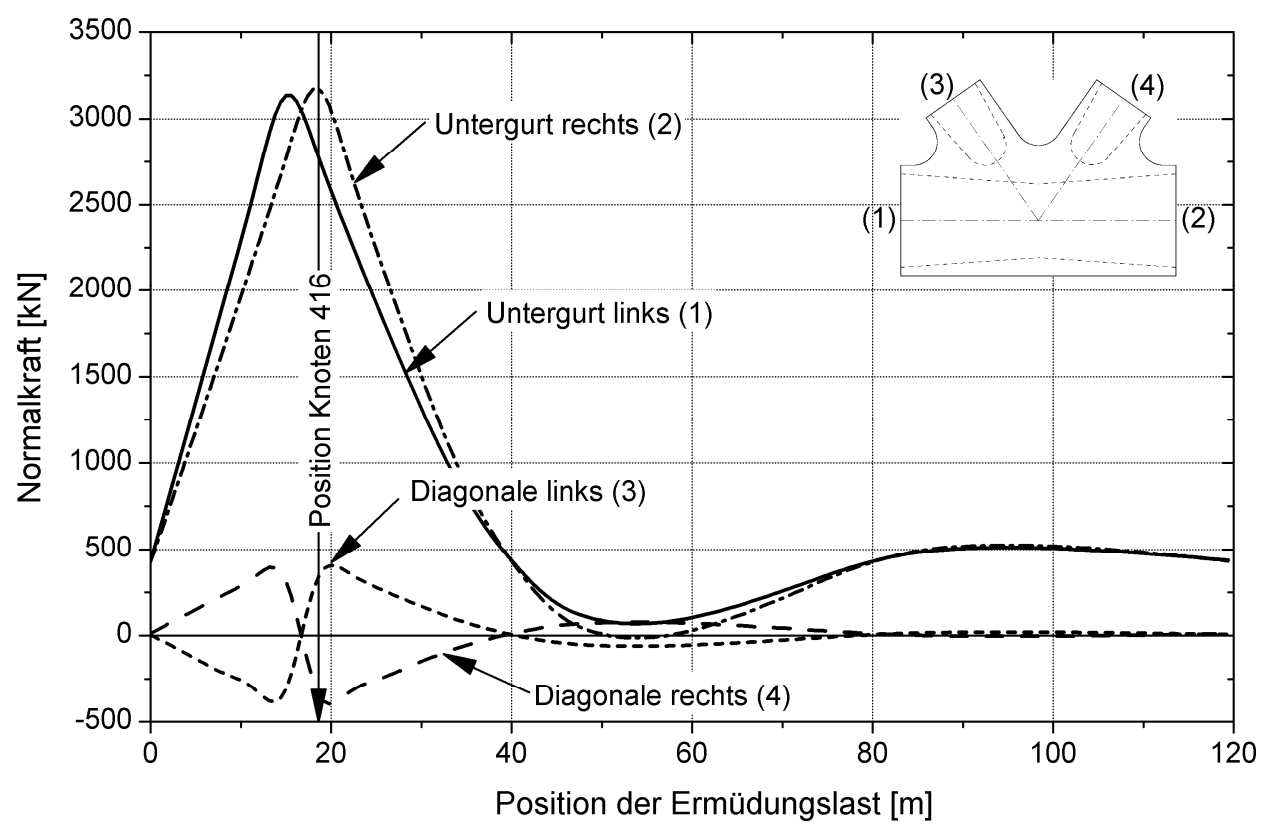

Fig. 5.9: Einflusslinien der an den Knoten 416 angreifenden Axialkräfte

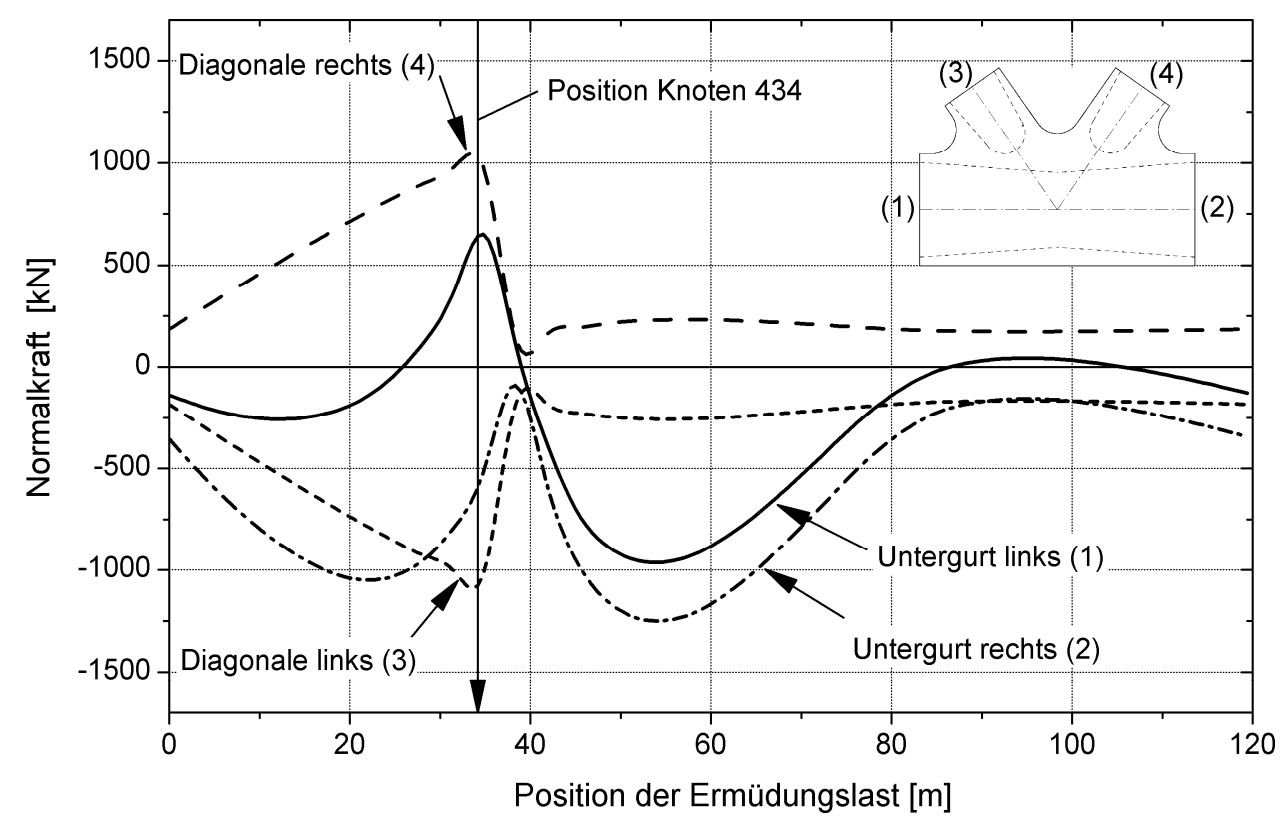

Fig. 5.10: Einflusslinien der an den Knoten 434 angreifenden Axialkräfte

Für die Simulation des Rissfortschritts müsste für ein einmaliges Überfahren der Ermüdungslast die zeitliche Veränderung der Belastung an allen Stutzenenden berücksichtigt werden. Nur so kann die Variation des für den SIF an der Rissfront massgebenden räumlichen Spannungszustands korrekt berücksichtigt werden. Mit dem Programm BEASY sind Variationen der Schnittkräfte an allen Stutzenenden nicht durchführbar. Daher wird für die Ermüdungsbelastung der Knoten eine Vereinfachung angestrebt. Sie soll darin bestehen, dass für jede zu untersuchende Fehlerstelle zwei Belastungszustände definiert werden, unter welchen die minimalen und maximalen SIF auftreten und die somit die effektiv auftretenden Spannungszustände gut abdecken. 
Um den Einfluss des variierenden Spannungszustandes auf die SIF zu untersuchen, werden die in Fig. 5.11 schematisch dargestellten 9 Fehlerstellen im Knoten ausgewählt. Für den Knoten 416 werden alle Fehlerstellen untersucht. Durch die Wärmebehandlung der Stahlgussknoten werden giesstechnisch bedingte Eigenspannungen zwar abgebaut, bei der Fabrikation des Fachwerkträgers können aber neue Eigenspannungen erzeugt werden. Obwohl die Untergurtstutzen im Knoten 434 unter Ermüdungslast ausschliesslich einer Druckbelastung ausgesetzt sind, kann Risswachstum nicht ausgeschlossen werden. Da für den Knoten 434 jedoch die Variation der Axialkraft im Untergurt im Vergleich zum Knoten 416 viel kleiner ist (vergleiche Fig. 5.9 und Fig. 5.10), ist das Risswachstum in den Untergurtstutzen des Knotens 416 für die Lebensdauer der Brücke massgebend. Aus diesem Grund werden für den Knoten 434 nur die Stellen 7-9 untersucht.

Durch die Wahl dieser Fehlerstellen werden sechs für zerstörungsfreie Untersuchungen gut zugängliche Stellen untersucht: 1-4, 8 und 9. Die Stellen 5-7 sind für Magnetpulver- oder Farbeindringprüfung schwer zugänglich und auch mit Ultraschall sehr schwierig zu untersuchen, da hier die Rückfallechos durch eine fehlende parallele Wand nicht reflektiert sondern im Knoten verstreut werden.

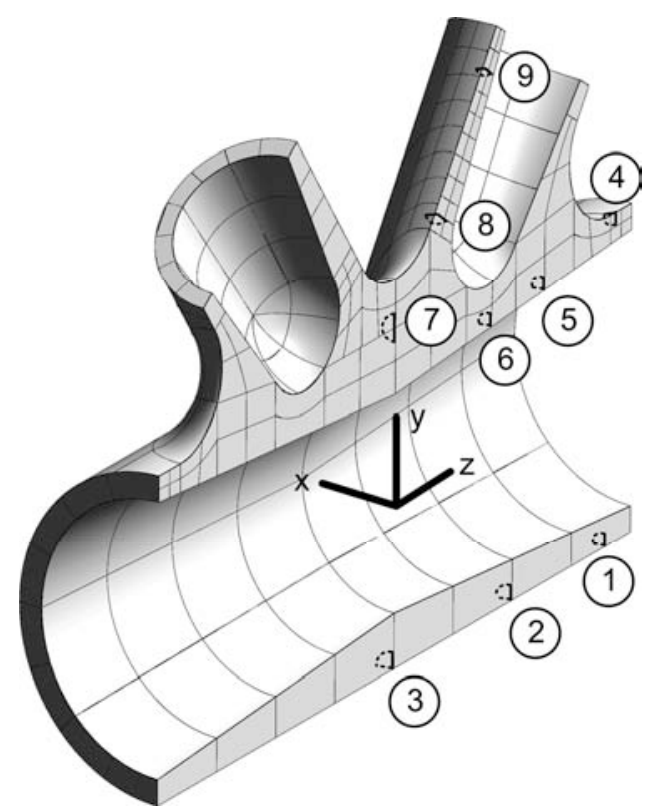

Fig. 5.11: Untersuchte Fehlerstellen im Knoten (Knoten 416: Nr. 1-9, Knoten 434: Nr. 7-9)

Für die Risswachstumssimulation eines von einer Fehlerstelle ausgehenden Risses ist die Differenz des Spannungsintensitätsfaktors $\Delta K$ notwendig, die aus den minimalen und maximalen SIF $\left(K_{\min }\right.$ bzw. $K_{\max }$ ) berechnet wird. Zur Bestimmung dieser SIF wird einheitlich für alle zu untersuchenden Fehlerstellen mit Ausnahme der Stelle 7 einheitlich ein halbkreisförmiger Oberflächenriss $(a=c)$ mit einer Rissgrösse $a=1.0 \mathrm{~mm}$ angenommen. An der Fehlerstelle 7 wird ein kreisförmiger Innenriss, ebenfalls mit einer Rissgrösse $a=1.0 \mathrm{~mm}$, eingesetzt. Infolge einer einmaligen Überfahrt der Ermüdungslast über die Brücke werden die SIF $K_{\mathrm{I}}, K_{\mathrm{II}}$ und $K_{\mathrm{III}}$ an der jeweiligen Fehlerstelle aus den Schnittkräften des Stabmodells der Fachwerkbrücke (Fig. 5.9 und Fig. 5.10) berechnet. Zwecks Vereinfachung der Berechnungen im BEASY-Modell werden die sehr kleinen Querkräfte vernachläs- 
sigt. Durch die Einführung eines einheitlichen Oberflächenrisses können die SIF qualitativ miteinander verglichen werden. Nachfolgend wird auf die einzelnen Fehlerstellen genauer eingegangen.

\section{Knoten 416: Fehlerstelle 1}

Da die Einflusslinien der SIF für die Fehlerstellen 1 bis 3 sehr ähnlich sind, wird hier nur die Fehlerstelle 1 dargestellt. Anhand von Fig. 5.12 kann Folgendes festgestellt werden:

- Es herrscht ein einachsiger Spannungszustand, da $K_{\text {II }}$ und $K_{\text {III }}$ annähernd null sind.

- $K_{\mathrm{I}}$ wird hauptsächlich durch die Zugkraft im Untergurt beeinflusst und variiert um den durch das Eigengewicht hervorgerufenen Wert $K_{\mathrm{I} \text {,Eigengewicht }}$.

- Der zum Maximum $K_{\mathrm{I}, \text { max }}$ gehörende Belastungszustand ist die Position der Ermüdungslast bei $18.6 \mathrm{~m}$; für $K_{\mathrm{I}, \min }$ ist es die Position bei $54 \mathrm{~m}$. Da $K_{\mathrm{I}, \min }$ im Verhältnis zu $K_{\mathrm{I}, \max }$ sehr klein ist und aus diesem Grund die Risswachstumssimulation mit nur einem Lastfall durchgeführt werden soll, wird $K_{\mathrm{I}, \mathrm{min}}$ mit einem Multiplikationsfaktor $\kappa$ wie folgt berücksichtigt:

$$
\kappa=\frac{K_{\mathrm{I}, \max }-K_{\mathrm{I}, \min }}{K_{\mathrm{I}, \max }}
$$

Daraus ergibt sich die Differenz $\Delta K_{\mathrm{I}}=\kappa \cdot K_{\mathrm{I}, \max }$ mit $\kappa=0.98$.

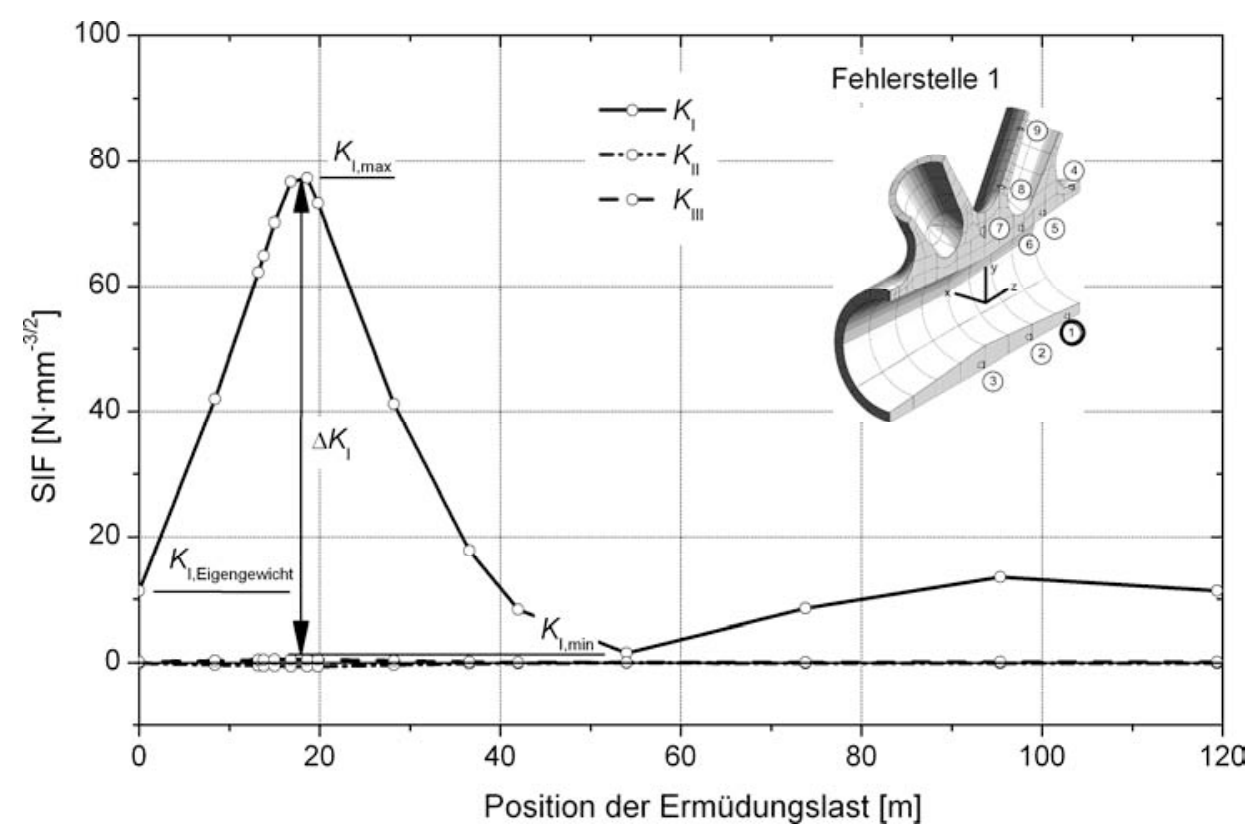

Fig. 5.12: Einflusslinien für $K_{\mathrm{I}}, K_{\mathrm{II}}$ und $K_{\mathrm{III}}$ für die Fehlerstelle 1, Knoten 416 


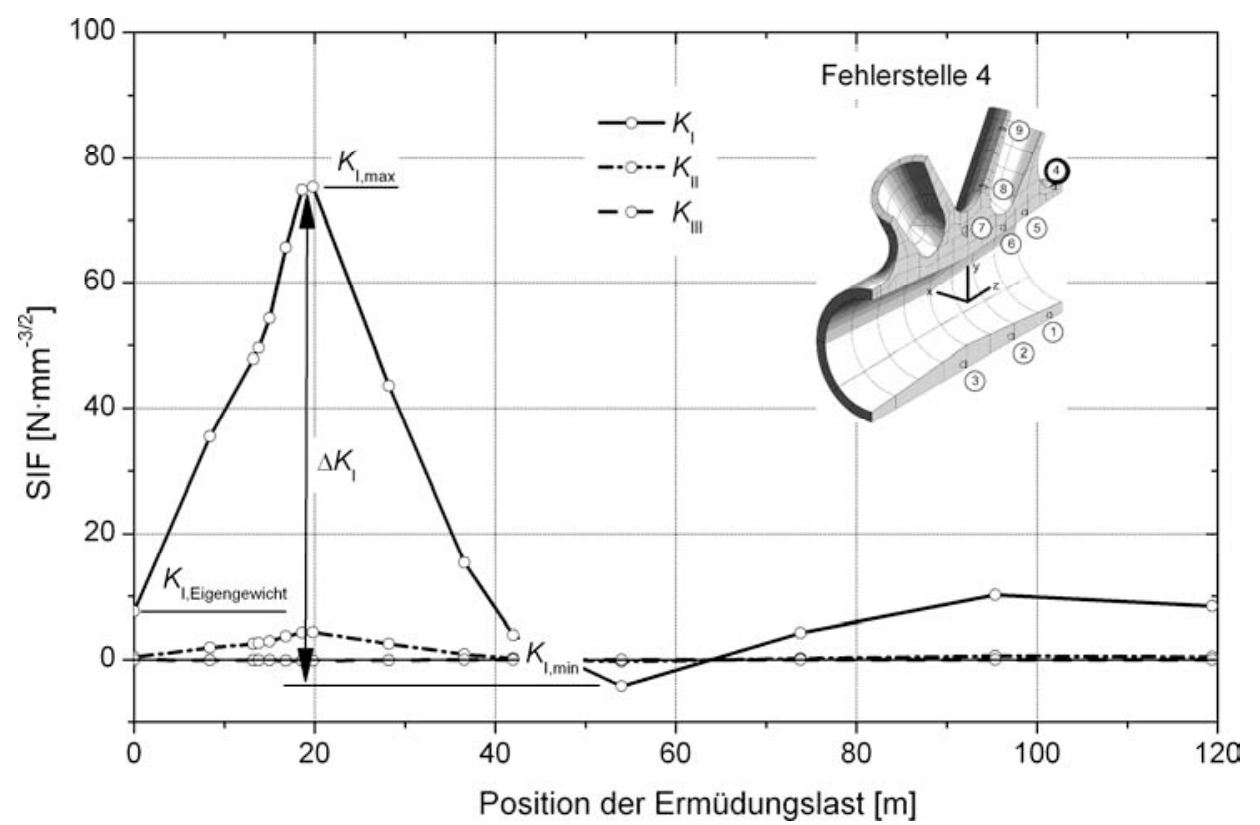

Fig. 5.13: Einflusslinien für $K_{\mathrm{I}}, K_{\mathrm{II}}$ und $K_{\mathrm{III}}$ für Fehlerstelle 4, Knoten 416

\section{Knoten 416: Fehlerstelle 4}

Da die Einflusslinien der SIF für die Fehlerstellen 4 bis 6 sehr ähnlich sind, wird hier nur die Fehlerstelle 4 dargestellt. Anhand von Fig. 5.13 kann Folgendes festgestellt werden:

- Es herrscht ein einachsiger Spannungszustand, da $K_{\text {II }}$ und $K_{\text {III }}$ annährend null sind.

- $K_{\mathrm{I}}$ wird hauptsächlich durch die Zugkraft im Untergurt beeinflusst.

- Der zum Maximum $K_{\mathrm{I}, \max }$ gehörende Belastungszustand ist die Position der Ermüdungslast bei $18.6 \mathrm{~m}$; für $K_{\mathrm{I} \text {,min }}$ ist es die Position bei $54 \mathrm{~m}$. Da auch für diese Stelle $K_{\mathrm{I}, \min }$ im Verhältnis zu $K_{\mathrm{I}, \max }$ sehr klein ist und aus diesem Grund die Risswachstumssimulation mit nur einem Lastfall durchgeführt soll, wird $K_{\mathrm{I}, \text { min }}$ ebenfalls mit einem Multiplikationsfaktor $\kappa$ nach Gleichung (5.1) berücksichtigt. Daraus ergibt sich die Differenz $\Delta K_{\mathrm{I}}=\kappa \cdot K_{\mathrm{I}, \max }=1.06$.

\section{Knoten 416: Fehlerstelle 7}

In Fig. 5.14 werden die Einflusslinien für die SIF an der Fehlerstelle 7 dargestellt. Folgendes kann festgestellt werden:

- Für einen vertikalen Riss (Neigung $0^{\circ}$ ) herrscht ein mehrachsiger Spannungszustand, der sich durch die SIF $K_{\mathrm{I}}$ und $K_{\text {III }}$ auszeichnet. Beim Wert von $K_{\text {III }}$ sind deutlich die Einflüsse der Normalkräfte in den Diagonalstutzen zu erkennen. $K_{\mathrm{I}}$ wird hauptsächlich durch die Zugkraft im Untergurt beeinflusst.

- Die SIF sind im Vergleich zu den Fehlerstellen 1 und 4 sehr niedrig.

- Die maximale Hauptspannung ist senkrecht zum Riss, wenn dieser um $-25^{\circ}$ gedreht ist.

- Der zum Maximum $K_{\mathrm{I}, \max }$ gehörende Belastungszustand bei der Rissstellung mit $-25^{\circ}$ ist die Position der Ermüdungslast bei $13.8 \mathrm{~m}$; für $K_{\mathrm{I}, \text { min }}$ ist es die Position bei $54 \mathrm{~m}$. Da auch für diese Stelle $K_{\mathrm{I}, \text { min }}$ im Verhältnis zu $K_{\mathrm{I} \text {,max }}$ sehr klein ist und aus diesem Grund die Risswachstumssimulation mit nur einem Lastfall durchgeführt soll, wird $K_{\mathrm{I}, \mathrm{min}}$ wieder mit einem Multiplikationsfaktor $\kappa$ nach Gleichung (5.1) berücksichtigt. Daraus ergibt sich die Differenz $\Delta K_{\mathrm{I}}=\kappa \cdot K_{\mathrm{I}, \max }=0.94$. 


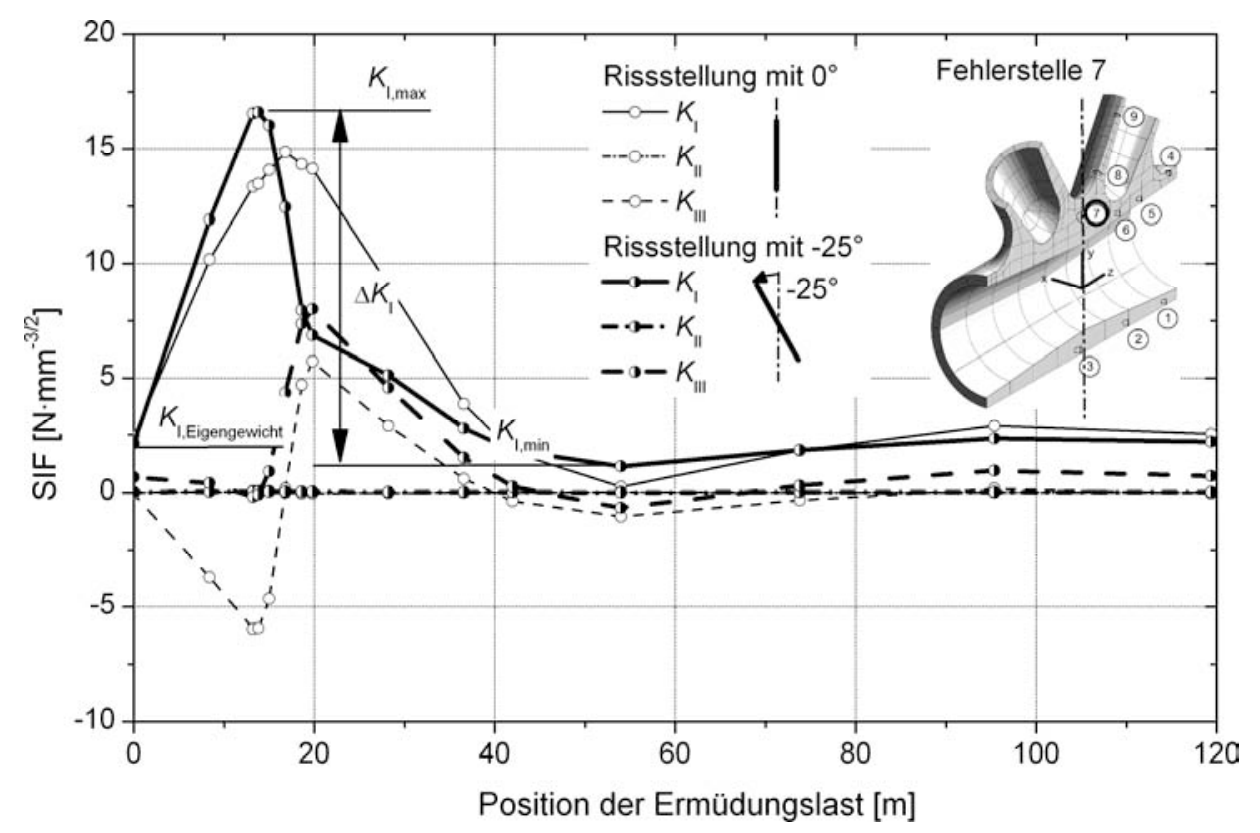

Fig. 5.14: Einflusslinien für $K_{\mathrm{I}} K_{\text {II }}$ und $K_{\text {III }}$ für Fehlerstelle 7, Knoten 416

\section{Knoten 416: Fehlerstelle 8}

Da die Einflusslinien der SIF für die Fehlerstellen 8 und 9 sehr ähnlich sind, wird hier nur die Fehlerstelle 8 dargestellt. Anhand von Fig. 5.15 kann Folgendes festgestellt werden:

- Es herrscht ein einachsiger Spannungszustand, da die SIF $K_{\text {II }}$ und $K_{\text {III }}$ annährend null sind.

- $K_{\mathrm{I}}$ wird hauptsächlich durch die Zug- und Druckkraft im Diagonalstutzen beeinflusst. Im Gegensatz zum Untergurtstutzen sind in diesem Knoten die Normalkräfte in den Diagonalstutzen infolge Eigengewicht und damit auch $K_{\mathrm{I} \text {,Eigengewicht }}$ vernachlässigbar klein.

- Der Belastungszustand $L C 1$ für $K_{\mathrm{I}, \text { max }}$ ist die Position der Ermüdungslast bei $13.8 \mathrm{~m}$; für $K_{\mathrm{I}, \mathrm{min}}$ ist der Belastungszustand LC2 die Position der Ermüdungslast bei $19.8 \mathrm{~m}$. Auf Grund möglicher Eigenspannungen im Knoten trägt $K_{\mathrm{I} \text {,min }}$ ebenfalls zum Risswachstum bei. Darum werden die zwei Belastungszustände $L C 1$ und $L C 2$ verwendet, um die Differenz des Spannungsintensitätsfaktors zu bestimmen, woraus sich $\Delta K_{\mathrm{I}}=K_{\mathrm{I}, \max }-K_{\mathrm{I}, \min }$ ergibt.

\section{Knoten 434: Fehlerstelle 7}

In Fig. 5.16 werden die Einflusslinien für die SIF $K_{\mathrm{I}}, K_{\mathrm{II}}$ und $K_{\mathrm{III}}$ für die Fehlerstelle 7 dargestellt. Folgendes kann festgestellt werden:

- Für eine vertikale Rissstellung (Neigung $0^{\circ}$ ) herrscht ein mehrachsiger Spannungszustand, der sich durch negative SIF $K_{\mathrm{I}}$ und insbesondere $K_{\text {III }}$ auszeichnet.

- Die SIF sind im Vergleich zur Fehlerstelle 8 (siehe unten) sehr niedrig.

- Die maximale Hauptspannung wirkt senkrecht zum Riss, wenn dieser um $-45^{\circ}$ gedreht ist.

Der zum Maximum $K_{\mathrm{I}, \max }$ gehörende Belastungszustand bei der Rissstellung mit $-45^{\circ}$ ist die Position der Ermüdungslast bei $33.6 \mathrm{~m}$; für $K_{\mathrm{I}, \mathrm{min}}$ ist es die Position bei $39.6 \mathrm{~m}$.

Da $K_{\mathrm{I}, \min }=0 \mathrm{~N} \cdot \mathrm{mm}^{-3 / 2}$ ergibt sich $\Delta K_{\mathrm{I}}=K_{\mathrm{I}, \text { max }}$. 


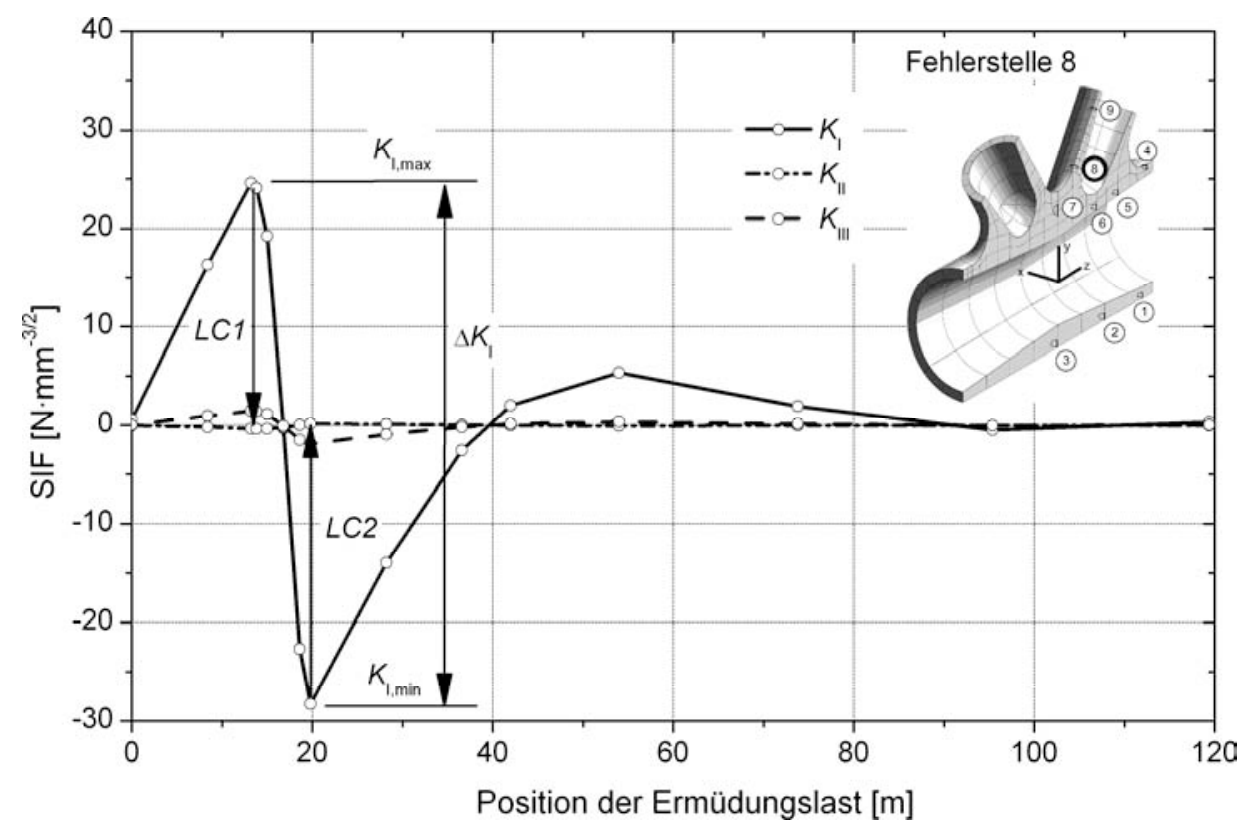

Fig. 5.15: Einflusslinien für $K_{\mathrm{I}}, K_{\mathrm{II}}$ und $K_{\mathrm{III}}$ für Fehlerstelle 8, Knoten 416

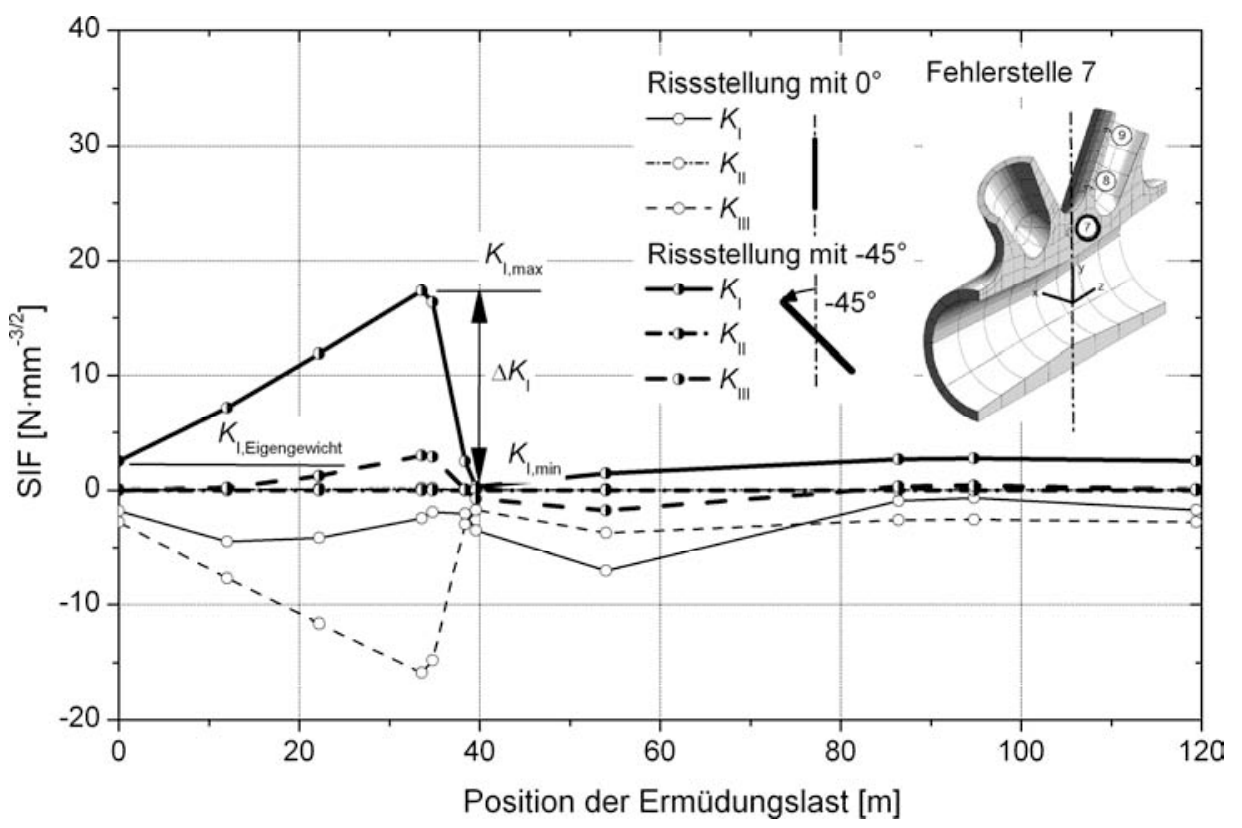

Fig. 5.16: Einflusslinien für $K_{\mathrm{I}}, K_{\mathrm{II}}$ und $K_{\mathrm{III}}$ für Fehlerstelle 7, Knoten 434

\section{Knoten 434: Fehlerstelle 8}

Da die Einflusslinien der SIF für die Fehlerstellen 8 und 9 sehr ähnlich sind, wird hier nur die Fehlerstelle 8 dargestellt. Anhand von Fig. 5.17 kann Folgendes festgestellt werden:

- Es herrscht ein einachsiger Spannungszustand, da die SIF $K_{\text {II }}$ und $K_{\text {III }}$ annährend null sind.

- $K_{\mathrm{I}}$ wird hauptsächlich durch die Zugkraft im Diagonalstutzen beeinflusst. Da sich der Knoten 434 nahe beim Auflager befindet, werden durch das Eigengewicht in den Diagonalstutzen Normalkräfte und damit $K_{\mathrm{I} \text {,Eigengewicht }}$ hervorgerufen.

- Der zum Maximum $K_{\mathrm{I}, \max }$ gehörende Belastungszustand ist die Position der Ermüdungslast bei $33.6 \mathrm{~m}$; für $K_{\mathrm{I}, \min }$ ist es die Position bei $39.6 \mathrm{~m}$. Da für diese Stelle $K_{\mathrm{I}, \min }$ im Verhältnis zu $K_{\mathrm{I}, \max }$ 
sehr klein ist und aus diesem Grund die Risswachstumssimulation mit nur einem Lastfall durchgeführt soll, wird $K_{\mathrm{I}, \mathrm{min}}$ mit dem Multiplikationsfaktor $\kappa$ nach Gleichung (5.1) berücksichtigt. Daraus ergibt sich die Differenz $\Delta K_{\mathrm{I}}=\kappa \cdot K_{\mathrm{I}, \max }=0.93$.

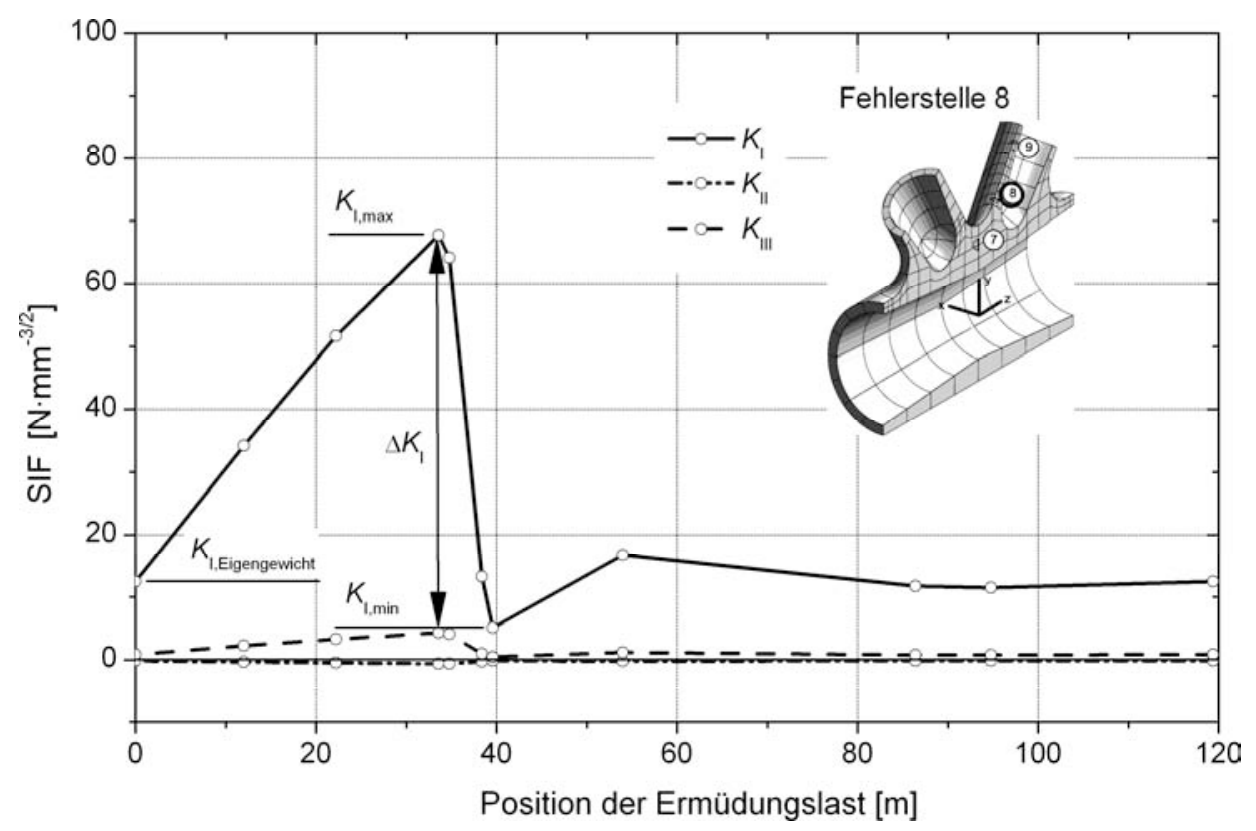

Fig. 5.17: Einflusslinien für $K_{\mathrm{I}}, K_{\mathrm{II}}$ und $K_{\mathrm{III}}$ für Fehlerstelle 8, Knoten 434

\subsection{RÜCKRECHNUNG AUF MAXIMAL ZULÄSSIGE ANFANGSFEHLERGRÖSSE}

\subsubsection{Berechnungsannahmen}

- Lebensdauer: Die Nutzungsdauer der Stahlgussknoten soll analog zu den Schweissnähten zwischen den Knotenstutzen und den Hohlprofilen 70 Jahre nach [SIA 261] betragen. Deshalb wird analog zu den Schweissnähten für die Stahlgussknoten ebenfalls eine charakteristische Lebensdauer von $N_{\text {tot }}=2 \cdot 10^{6}$ Lastwechseln gewählt.

- Langriss-Bruchmechanik: Das Kurzrissstadium des Ermüdungsvorgangs wird bei der Rückrechnung nicht mitberücksichtigt, sondern die Ersatzfehler für die Gussfehler werden als Makrorisse oder so genannte Langrisse modelliert und ihr Wachstum wird entsprechend simuliert (Abschnitt 2.3.1.4).

- Deterministische Berechnung: Sämtliche Berechnungen werden deterministisch durchgeführt, auf probabilistische Betrachtungen wird im Rahmen dieser Arbeit verzichtet. Für die Einwirkungen werden die charakteristischen Werte mit den dazugehörigen Lastbeiwerten nach der Norm [SIA 261] angesetzt. Für die Materialkennwerte des Stahlgusses werden die charakteristischen Werte für den GS-20 Mn 5 nach [DIN 17182:1992] herangezogen. Die Rissfortschrittsparameter $C$ und $m$ für ferritisch-perlitische Stähle, zu denen auch der Stahlguss GS-20 Mn 5 zählt, werden nach [Barsom 1971] (in [Barsom et al. 1999] übernommen) angesetzt. Diese Werte entsprechen einer konservativen Auswertung von Versuchsresultaten.

- Bruchzähigkeit: Schlag- oder stossartige Beanspruchungen der Stahlgussknoten werden ausgeschlossen. Die für den Sprödbruch massgebende Bruchzähigkeit ist somit die quasi-statische Bruchzähigkeit. 


\subsubsection{Vorgehen}

Das Prinzip der Rückrechnung wird in Fig. 5.18 schematisch dargestellt. Die einzelnen Etappen sind nachfolgend beschrieben.
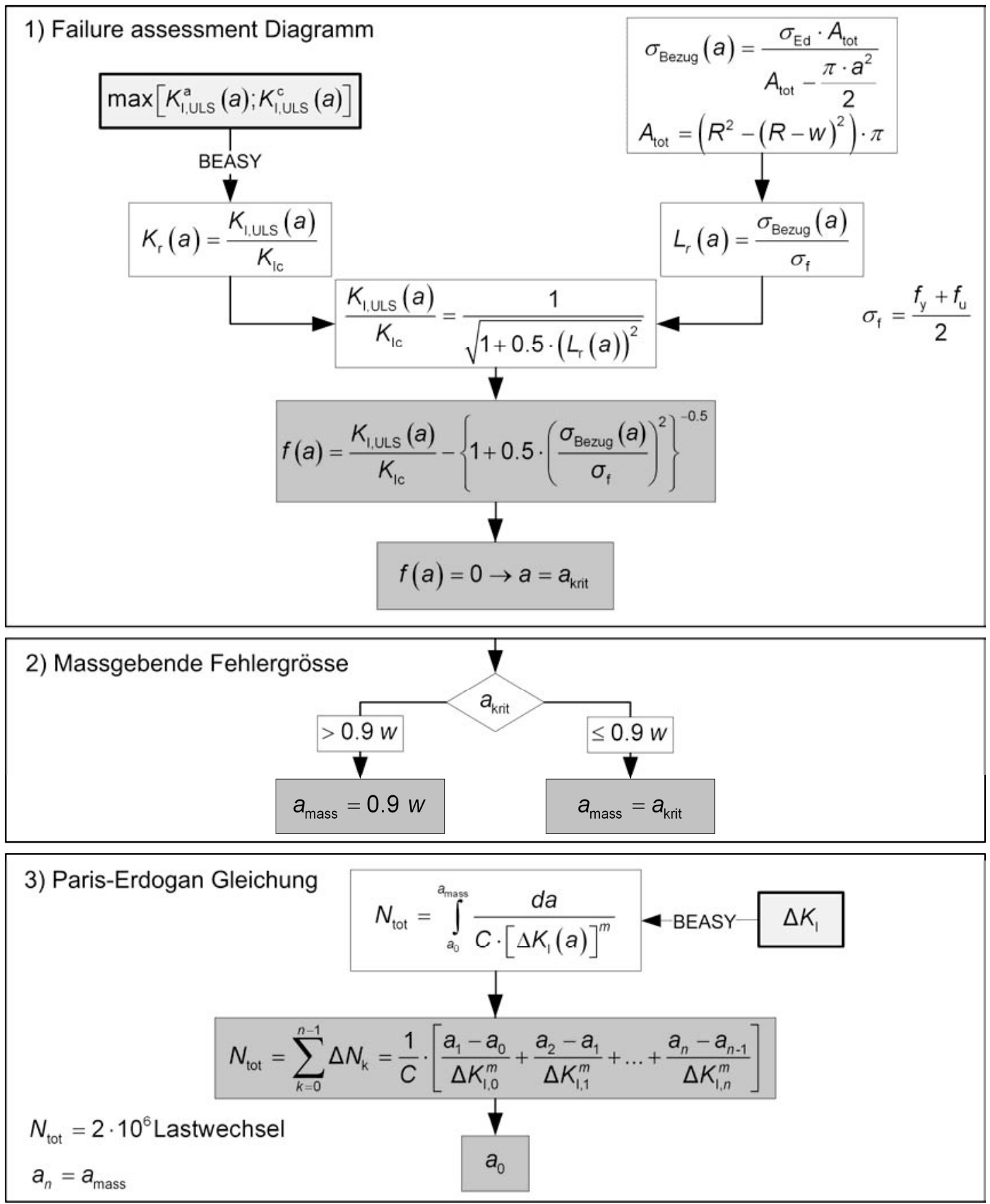

Fig. 5.18: Prinzip der Rückrechnung

\section{1) Failure Assessment Diagramm}

Ein Kriterium für die massgebende Fehlergrösse (siehe folgender Abschnitt) ist die kritische Fehlergrösse $a_{\text {krit, }}$ bei der das Bauteil durch Sprödbruch versagt. Sie entspricht der Risstiefe, bei der unter statischer Belastung (ULS) der Spannungsintensitätsfaktor an der wachsenden Rissfront einer Fehlerstelle die Bruchzähigkeit des Stahlgusses erreicht: 


$$
K_{\mathrm{I}, \mathrm{ULS}}\left(a_{\text {krit }}\right)=K_{\mathrm{Ic}}
$$

$K_{\text {Ic }} \quad$ Bruchzähigkeit des Stahlgusses bei $T=-30^{\circ} \mathrm{C}, K_{\mathrm{Ic}}=2400 \mathrm{~N} \cdot \mathrm{mm}^{-3 / 2}($ Abschnitt 3.2.2)

$K_{\mathrm{I}, \mathrm{ULS}}\left(a_{\text {krit }}\right) \quad$ SIF unter statischer Belastung bei der kritischen Risstiefe $a_{\text {krit }}$

Der SIF unter statischer Belastung bei der für Sprödbruch kritischen Risstiefe $a_{\text {krit }}$ ist der maximale SIF entlang der Rissfront. Die zwei Extremwerte bei einem Oberflächenriss sind die Werte am Rissrand $K_{\mathrm{I}, \mathrm{ULS}}^{\mathrm{c}}\left(a_{\text {krit }}\right)$ und in der Risstiefe $K_{\mathrm{I}, \mathrm{ULS}}^{\mathrm{a}}\left(a_{\text {krit }}\right)$. Daraus ergibt sich:

$$
K_{\mathrm{I}, \mathrm{ULS}}\left(a_{\text {krit }}\right)=\max \left[K_{\mathrm{I}, \mathrm{ULS}}^{\mathrm{a}}\left(a_{\text {krit }}\right) ; K_{\mathrm{I}, \mathrm{ULS}}^{\mathrm{c}}\left(a_{\text {krit }}\right)\right]
$$

Die Bruchzähigkeit des Materials hängt vom Spannungszustand um die Rissspitze herum ab. Am Rissrand auf der Oberfläche herrscht ein ebener Spannungszustand, der gegen die Risstiefe hin in einen ebenen Dehnungszustand übergeht. Je ausgeprägter der dreiaxiale Spannungszustand (ebener Dehnungszustand) wird, desto kleiner wird die plastische Zone um die Rissspitze und desto mehr nähert sich die Bruchzähigkeit des Materials ihrem Minimum $K_{\mathrm{Ic}}$. Auf der Oberfläche liegt also ein ebener Spannungszustand, eine grosse plastische Zone um den Rissrand und eine Bruchzähigkeit, die höher ist als $K_{\text {Ic }}$ vor. Entsprechend ist Gleichung (5.3) konservativ, wenn der maximale SIF am Rissrand auftritt und dieser mit der Bruchzähigkeit $K_{\mathrm{Ic}}$ für einen ebenen Dehnungszustand verglichen wird.

Wenn die Tragfähigkeit des Querschnitts und der Spannungsfluss im abgeschwächten Querschnitt durch den vorhandenen Riss beeinträchtigt werden, muss die kritische Fehlergrösse für Sprödbruch im Failure Assessment Diagramm (FAD) [Milne et al. 1986] [BS 7910:1999] bestimmt werden, um einem eventuellen plastischen Bruch Rechnung zu tragen. Die Grenzkurve für Werkstoffe mit ausgeprägter Fliessgrenze sowie Lüdersdehnung wird folgendermassen umschrieben (Fig. 5.19):

$$
K_{\mathrm{r}}=\left\{\begin{array}{ccc}
K_{\mathrm{r}}=\frac{1}{\sqrt{1+0.5 \cdot L_{\mathrm{r}}^{2}}} & \text { für } & L_{\mathrm{r}} \leq 1.0 \\
0 & \text { für } & L_{\mathrm{r}}>1.0
\end{array}\right.
$$

Die Bewertung der Bauteilsicherheit beruht auf dem bruchmechanischen Konzept. Die Bauteilbeanspruchung wird durch die zwei normierten Grössen charakterisiert:

$$
L_{\mathrm{r}}=\frac{\sigma_{\text {Bezug }}(a)}{\sigma_{\mathrm{f}}}
$$

$\sigma_{\text {Bezug }}(a) \quad$ Nominalspannung im Kreisquerschnitt mit Riss (Querschnitt abzüglich der Rissfläche mit $a / c=1.0$ )

$\sigma_{\mathrm{f}} \quad$ Mittelwert aus Fliessgrenze $f_{\mathrm{y}}$ und Zugfestigkeit $f_{\mathrm{u}}$

$$
K_{\mathrm{r}}=\frac{K_{\mathrm{I}}}{K_{\mathrm{Ic}}}
$$

$K_{\mathrm{I}} \quad$ Spannungsintensitätsfaktor; unter statischer Last $K_{\mathrm{I}}=K_{\mathrm{I}, \mathrm{ULS}}$ nach Gleichung (5.3)

$K_{\text {Ic }} \quad$ Bruchzähigkeit

Das Versagen des Bauteils tritt ein, wenn bei einer gegebenen Belastung der Punkt mit den Koordinaten $\left(L_{\mathrm{r}}, K_{\mathrm{r}}\right)$ auf oder ausserhalb der Grenzkurve liegt. Je nach Zunahme der Spannung $\sigma$, der Streckgrenze $f_{\mathrm{y}}$, der Risstiefe $a$ und der Bruchzähigkeit $K_{\mathrm{Ic}}$ verschiebt sich der Koordinatenpunkt $\left(L_{\mathrm{r}}, K_{\mathrm{r}}\right)$ in die entsprechende Richtung (Fig. 5.19). Sind keine Eigenspannungen im Bauteil vorhanden, liegt für ein unbelastetes Bauteil der Koordinatenpunkt bei $(0,0)$. 


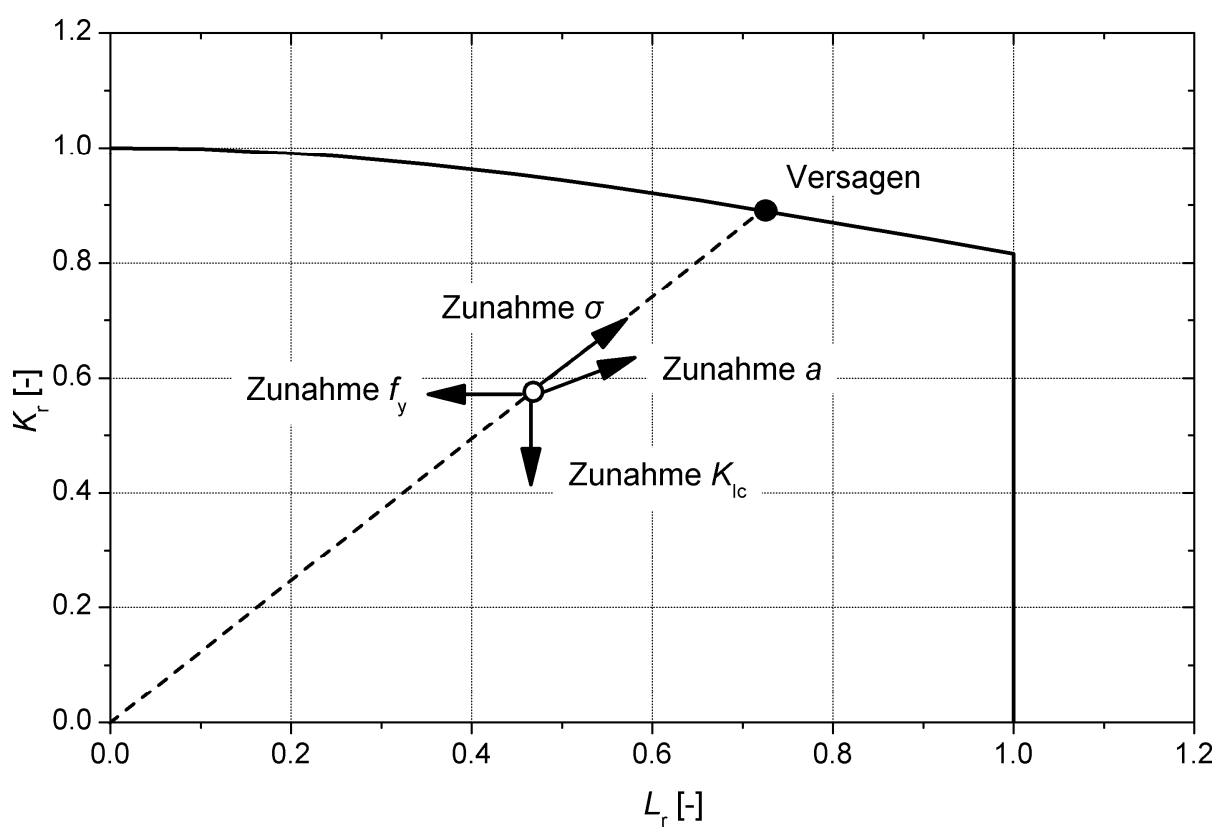

Fig. 5.19: Bruchsicherheitsbewertung auf der Basis des FAD

\section{2) Massgebende Fehlergrösse}

Die massgebende Fehlergrösse $a_{\text {mass }, i}$ an der Stelle $i$ für die Rückrechnung auf die maximal zulässige Anfangsfehlergrösse $a_{0, i}$ wird wie folgt festgelegt: Ein Wanddurchbruch des Risses wird als nicht tolerierbar eingestuft. Nach Abzug einer Marge von $10 \%$ folgt daraus das erste Kriterium, nämlich dass $a_{\text {mass }, i}$ kleiner sein muss als $90 \%$ der Wanddicke $w$. Das zweite Kriterium folgt direkt aus dem Sprödbruchnachweis (siehe folgender Abschnitt), indem $a_{\text {mass }, i}$ kleiner sein muss als die kritische Risstiefe für Sprödbruch $a_{\text {krit. }}$ Die massgebende Fehlergrösse ist also $a_{\text {mass }, i}=\min \left(0.9 w ; a_{\text {krit }}\right)$.

Das beobachtete Risswachstum in den Schweissnähten der Versuchskörper (siehe Abschnitt 3.5.2) bestätigt die Tatsache, dass ab dem Eintreten eines Durchrisses die Risswachstumsgeschwindigkeit sehr steil ansteigt. Die Wachstumsphase der beobachteten Durchrisse trägt höchstens 10 \% zur gesamten Lebensdauer bei, bevor es zu Bruch kommt. Rissfortschrittsberechnungen mit BEASY an einer Schweissnaht zeigen zwar in Abschnitt 4.3.3 einen höheren Anteil von ungefähr $17 \%$ an der Lebensdauer, im weiteren Vorgehen wird dieser jedoch auf der sicheren Seite liegend nicht mitberücksichtigt.

\section{3) Paris-Erdogan Gleichung (linear elastische Bruchmechanik)}

Für die Rückrechnung auf die maximal zulässige Anfangsfehlergrösse $a_{0, i}$ wird die Paris-Erdogan Gleichung nach [Paris 1960] benutzt:

$$
\frac{\mathrm{d} a}{\mathrm{~d} N}=C \cdot \Delta K_{\mathrm{I}}(a)^{m}
$$

a Risstiefe im tiefsten Punkt des Risses

$N \quad$ Anzahl Lastwechsel; $N=2 \cdot 10^{6}$ (entspricht einer Nutzungsdauer der Stahlgussknoten von 70 Jahre nach [SIA 261])

$\Delta K_{\mathrm{I}}(a) \quad$ Differenz des Spannungsintensitätsfaktors bei Risstiefe $a$

$C, m \quad$ Rissfortschrittsparameter mit $C=2 \cdot 10^{-13}(\mathrm{~mm} /$ Lastwechsel $) \cdot\left(\mathrm{N} \cdot \mathrm{mm}^{-3 / 2}\right)^{-m}, m=3$

(siehe Abschnitt 2.3.1.3) 
Die Anfangsfehlergrösse $a_{0, i}$ an der Fehlerstelle $i$ lässt sich aus dem Integral ableiten:

$$
N=\int_{a_{0, i}}^{a_{\text {mass } i}} \frac{\mathrm{d} a}{C \cdot\left(\Delta K_{\mathrm{I}}(a)\right)^{m}}
$$

$a_{\text {mass }, i} \quad$ massgebende Fehlergrösse an der Fehlerstelle $i\left(a_{\text {mass }, i}=\min \left[0.9 w_{i} ; a_{\text {krit }, i}\right]\right)$

Auf Grund der diskreten Rechenergebnisse für $\Delta K_{\mathrm{I}}(a)$ aus BEASY kann das Integral in Gleichung (5.8) nicht direkt, sondern ebenfalls nur diskret gelöst werden (siehe Fig. 5.18, dritter Schritt).

Risse, deren $\Delta K(a)$ unter dem Schwellenwert $\Delta K_{\text {th }}$ liegt, wachsen nicht. Wenn $\Delta K(a)<\Delta K_{\text {th }}$ gilt also

$$
\frac{\mathrm{d} a}{\mathrm{~d} N}=0 .
$$

In [Lampman et al. 1996] sind für C-Mn Stahlguss Werte für $\Delta K_{\text {th }}$ zwischen $120 \mathrm{~N} \cdot \mathrm{mm}^{-3 / 2}$ und $260 \mathrm{~N} \cdot \mathrm{mm}^{-3 / 2}$ angegeben. Für die Rückrechnung auf die Anfangsfehlergrösse $a_{0, i}$ wird hier die konservative Annahme von $\Delta K_{\text {th }}=0 \mathrm{~N} \cdot \mathrm{mm}^{-3 / 2}$ gemacht. Damit trägt jede Differenz des Spannungsintensitätsfaktors $\Delta K(a)$ zum Risswachstum bei.

Wenn $K_{\min }(a)$ kleiner ist als der Spannungsintensitätsfaktor $K_{\mathrm{op}}(a)$, bei dem die Risslippen tatsächlich geöffnet werden, ist die effektive Differenz des Spannungsintensitätsfaktors $\Delta K_{\text {eff }}(a)$, die zum Risswachstum führt, nur $K_{\max }(a)-K_{\mathrm{op}}(a)$ (Rissschliesseffekt). Es gilt also allgemein

$$
\Delta K(a)=\Delta K_{\mathrm{eff}}(a)=K_{\max }(a)-\max \left[K_{\mathrm{op}}(a) ; K_{\min }(a)\right] .
$$

Durch die Wärmebehandlung der Stahlgussknoten werden giesstechnisch bedingte Eigenspannungen zwar abgebaut, bei der Fabrikation des Fachwerkträgers können aber neue Eigenspannungen erzeugt werden. Auf Grund der möglicherweise vorhandenen Zugeigenspannungen kann $K_{\min }(a)$ grösser sein als $K_{\mathrm{op}}(a)$. Da der Zustand der (Zug-)Eigenspannung schwierig abzuschätzen ist, wird für die weiteren Untersuchungen die konservative Annahme gemacht, dass die effektive Differenz $\Delta K_{\text {eff }}(a)=K_{\max }(a)-K_{\min }(a)$ ist.

Die plastischen Zonen um die Rissspitzen an der Oberfläche, wo ein ebener Spannungszustand herrscht, können durch einen kreisförmigen Bereich mit dem Radius $r_{\mathrm{y}}$ beschrieben werden. Der Radius beträgt für den ebenen Dehnungszustand nach [McClintock et al. 1965]:

$$
r_{\mathrm{y}}=\frac{1}{6 \pi}\left(\frac{K_{\mathrm{I}}}{f_{\mathrm{y}}}\right)^{2}
$$

$r_{\mathrm{y}} \quad$ Radius der plastischen Zone im Bereich der Rissfront

$f_{\mathrm{y}} \quad$ Fliessgrenze; $f_{\mathrm{y}}=355 \mathrm{MPa}$

Unter Berücksichtigung der plastischen Zone um die Rissspitze ergibt sich die effektive Risstiefe

$$
a_{\mathrm{eff}}=a+r_{\mathrm{y}}
$$

und daraus die Differenz des effektiven Spannungsintensitätsfaktors $K_{\mathrm{I}}\left(a_{\mathrm{eff}}\right)$ :

$$
\Delta K_{\mathrm{I}}\left(a_{\mathrm{eff}}\right)=Y\left(a_{\mathrm{eff}}\right) \cdot \sigma \sqrt{\pi \cdot a_{\mathrm{eff}}}
$$

Die maximal zulässige Anfangsfehlergrösse $a_{0, i}$ müsste also um die plastische Zone korrigiert werden. Die SIF $K_{\mathrm{I}}$ zur Anfangsfehlergrösse $a_{0, i}$ (Tab. 5.3) sind jedoch so niedrig (vergleiche Fig. 5.21 und Fig. 5.22), dass der Radius der plastischen Zone maximal $0.14 \mathrm{~mm}$ beträgt (für Stelle 4, mit 
$a_{0}=17.7 \mathrm{~mm}$ und $\Delta K_{\mathrm{I}}=580 \mathrm{~N} \cdot \mathrm{mm}^{-3 / 2}$ ). In Anbetracht der aus den Berechnungen resultierenden hohen zulässigen Anfangsfehlergrössen und der beschränkten Genauigkeit der Simulationen ist die Korrektur vernachlässigbar.

\subsubsection{Simulation des Risswachstums}

\subsubsection{Vorgehen}

Die ausgewählten Fehlerstellen (Fig. 5.11) werden einzeln als Risse mit einer willkürlich gewählten Anfangsfehlergrösse, die generell etwa $5 \%$ der Wanddicke entspricht, in das BE-Modell eingefügt. Bei der automatischen Risswachstumssimulation werden die Initialrisse inkrementell ausgedehnt und die SIF für jedes Inkrement bestimmt. Für jedes Inkrement wird eine neue Eingabedatei (Angaben zu Knotengeometrie, Riss mit neuer Rissfront und Belastung) erstellt. Zunächst wird das Risswachstum unter der Ermüdungsbelastung simuliert, anschliessend werden für den Sprödbruchnachweis die Spannungsintensitätsfaktoren (SIF) für die bereits berechneten Rissfronten unter statischer Last bestimmt. Dazu werden in den erstellten Eingabedateien der einzelnen Inkremente die Knotenbelastung geändert und anschliessend die abgeänderten Eingabedateien neu berechnet. Anhand der Rechenergebnisse können anschliessend die Rückrechnungen gemäss Abschnitt 5.4.2 vorgenommen werden.

\subsubsection{Stahlgussfehler}

Für die SIF-Berechnungen werden die Gussfehler als halbkreisförmige Oberflächenrisse $(a / c=1.0)$ angenommen. Dabei handelt es sich in zweifacher Hinsicht um eine konservative Annahme. Einerseits verhalten sich Risse im Vergleich zu anderen Fehlerarten wie Lunker, Poren und Schlackeneinschlüsse (siehe Abschnitt 2.2.1) in Bezug auf das Rissfortschrittsverhalten aggressiver. Anderseits wächst ein Oberflächenriss im Vergleich zu einem Innenriss mit gleicher Rissgrösse auf Grund der höheren Spannungsintensitätsfaktoren in der Risstiefe schneller.

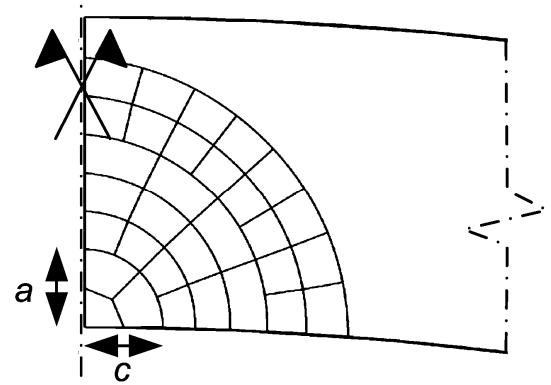

a) Halbkreisförmige Rissfront $(a=2 c)$

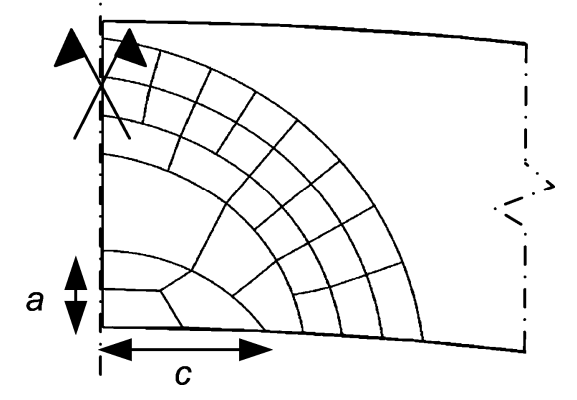

b) Halbelliptische Rissfront $(2 a=c)$

Fig. 5.20: Vergleich Rissfortschritt mit halbkreisförmiger und halbelliptischer Rissfront in einem Rohrquerschnitt

Die Wahl eines halbkreisförmigen statt eines halbelliptischen Oberflächenrisses ( $a / c \leq 1.0)$ beruht auf folgenden Überlegungen: Bei den Simulationen des Wachstums eines halbkreisförmigen Oberflächenrisses zeigt sich, dass das anfängliche Verhältnis von $a / c=1.0$ nur auf durchschnittlich 0.9 abfällt (Fig. 5.20 a). Ein halbelliptischer Oberflächenriss wächst anfangs schnell in Richtung der Risstiefe $a$, bis sich die Rissfront ebenfalls auf das Verhältnis von 0.9 einpendelt und dann so weiter wächst (Fig. 5.20 b). Wird für einen halbelliptischen Anfangsriss die Rissfront durch die entspre- 
chende halbkreisförmige Rissfront ersetzt, kann dieser mit dem halbkreisförmigen Riss abgedeckt werden.

Es wird hier nochmals darauf hingewiesen, dass nach der Norm [DIN 1690-2:1985] Risse für alle Gütestufen grundsätzliche nicht zulässig sind, sofern nicht durch bruchmechanische Untersuchungen ihre Unbedenklichkeit nachgewiesen wird. Wenn im Weiteren von Rissen die Rede ist, so handelt es sich immer um Ersatzfehler, die alle anderen Gussfehler wie Lunker, Gasporen, Schlauchporen und Schlackeneinschlüsse sicher abdecken.

\subsubsection{Ergebnisse der SIF-Berechnungen unter Ermüdungsbelastung}

Die Auswertung der Rechenergebnisse hat gezeigt, dass die Spannungsintensitätsfaktoren (SIF) in der Risstiefe $K_{\mathrm{I}}^{\mathrm{a}}$ in der Regel niedriger sind als die SIF am Rissrand $K_{\mathrm{I}}^{\mathrm{c}}$. Aus diesem Grund tendiert die Rissform mit dem anfänglichen Verhältnis $a / c=1.0$ auf ein Verhältnis von durchschnittlich 0.9. Da für die Rückrechnung auf die maximal zulässige Anfangsfehlergrösse die SIF unter Ermüdungsbelastung in der Risstiefe $K_{\mathrm{I}}^{\mathrm{a}}$ erforderlich sind, werden nachfolgend nur diese dargestellt.

In Fig. 5.21 werden die SIF $K_{\mathrm{I}, \max }$ unter der in Abschnitt 5.3.3 angegebenen Ermüdungsbelastung für alle Fehlerstellen des Knotens 416 zusammengestellt. Es ist zu erkennen, dass für die Fehlerstellen 1-5 im Vergleich zu den Stellen 6-9 die SIF höher ausfallen. Da bereits aus den Fig. 5.12, Fig. 5.13 und Fig. 5.15 ersichtlich ist, dass $K_{\text {II }}$ und $K_{\text {III }}$ annährend null sind, wird hier auf ihre Darstellung verzichtet. Die Berechnung der Differenz des Spannungsintensitätsfaktors $\Delta K_{\mathrm{I}}$ wird für alle Fehlerstellen in Abschnitt 5.3.3 erläutert.

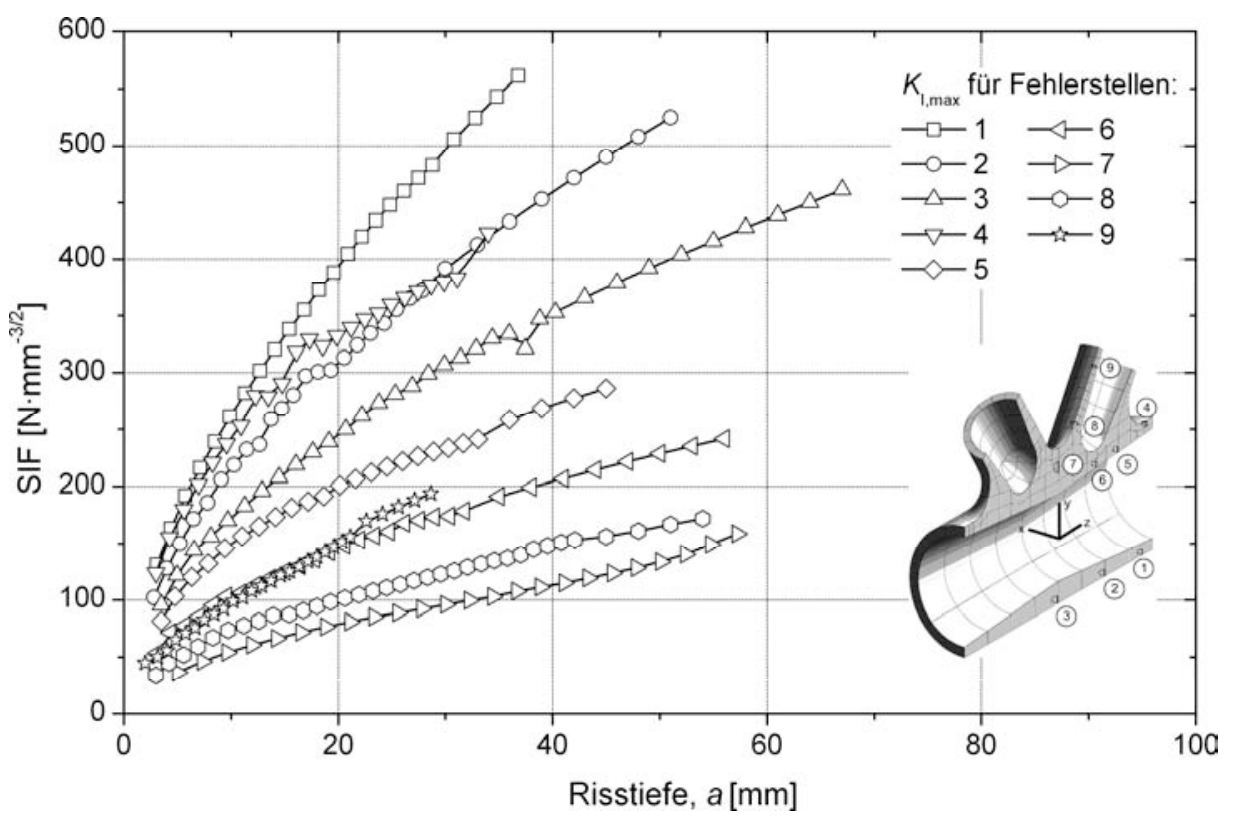

Fig. 5.21: Zusammenstellung von $K_{\mathrm{I}, \max }$ für alle Fehlerstellen im Knoten 416, unter Ermüdungslast

Wie bereits in Abschnitt 5.3.3 erwähnt, werden für den Knoten 434 nur die Fehlerstellen 7 - 9 betrachtet, da für diesen Knoten die Variation der Axialkraft im Untergurt im Vergleich zum Knoten 416 viel kleiner ist (vergleiche Fig. 5.9 und Fig. 5.10) und somit das Risswachstum in den Untergurtstutzen des Knotens 416 für die Lebensdauer der Brücke massgebend ist. Fig. 5.22 fasst für die drei Fehlerstellen die SIF $K_{\mathrm{I}, \text { max }}$ zusammen. Auch hier wird auf die Darstellung von $K_{\text {II }}$ und $K_{\text {III }}$ verzichtet, da bereits in Fig. 5.17 gezeigt wird, dass diese vernachlässigbar klein sind. 


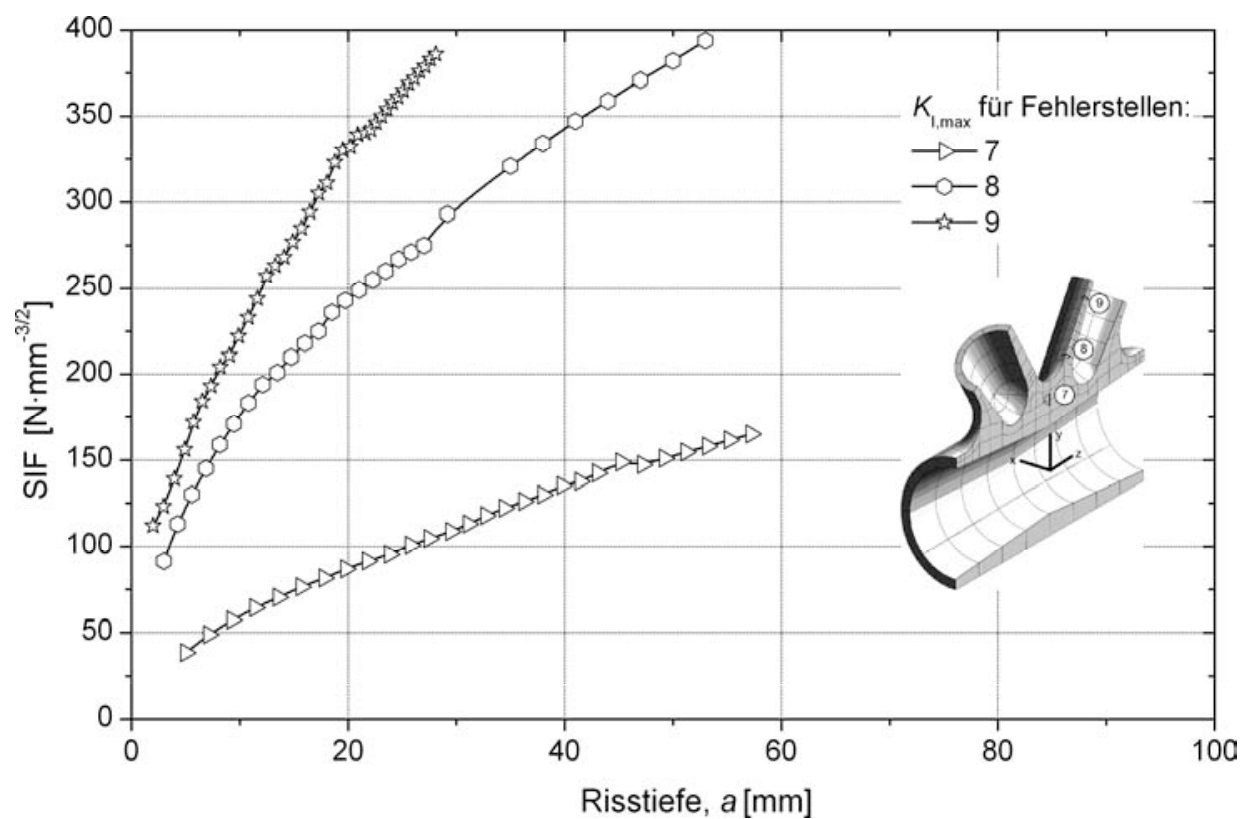

Fig. 5.22: Zusammenstellung von $K_{\mathrm{I}, \max }$ für alle Fehlerstellen im Knoten 434, unter Ermüdungslast

Für die Fehlerstelle 7 werden in Fig. 5.23 für beide Knoten nochmals alle SIF zusammengestellt. In Fig. 5.14 und Fig. 5.16 wird gezeigt, dass mit einer Drehung des Risses gegenüber der Vertikalen die SIF $K_{\text {III }}$ ab- und die SIF $K_{\mathrm{I}}$ zunehmen. Fig. 5.23 zeigt deutlich, dass bei einer Rissdrehung von $-25^{\circ}$ im Knoten 416 und $-45^{\circ}$ im Knoten 434 ein einachsiger Spannungszustand herrscht.

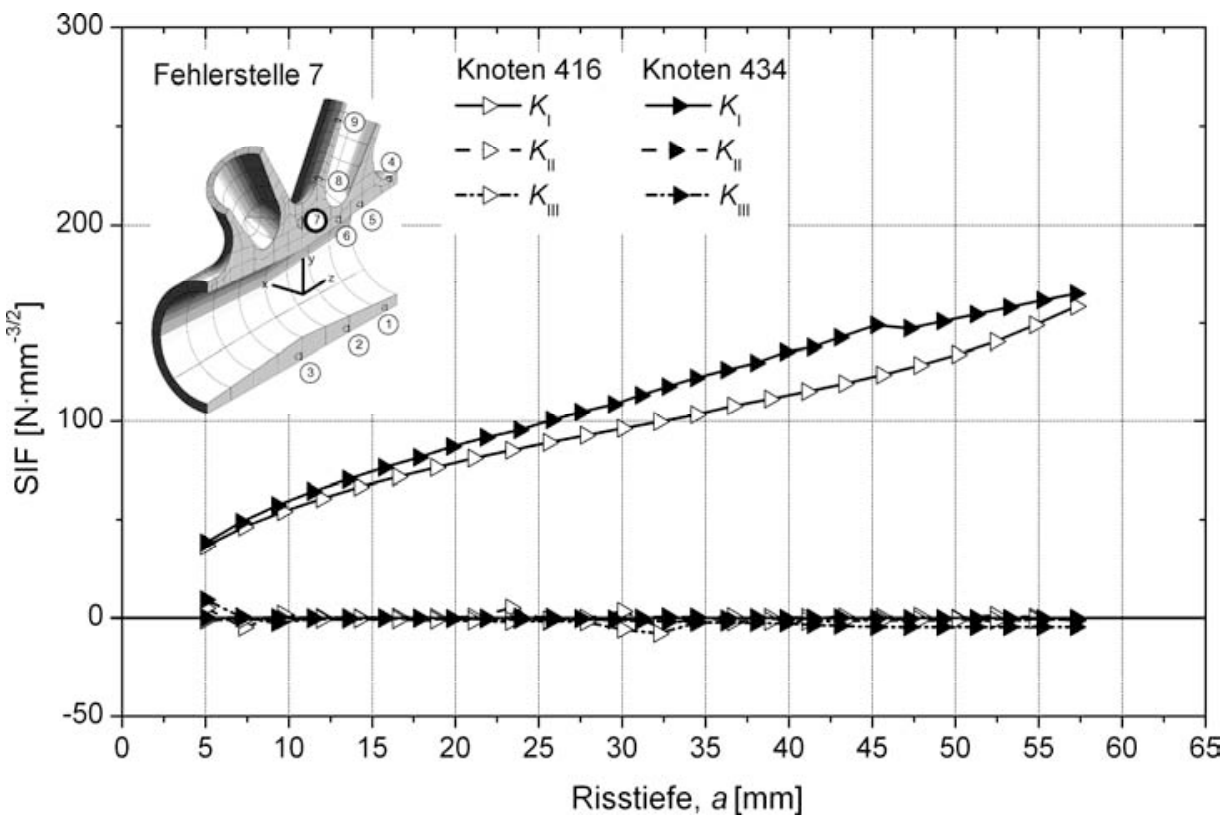

Fig. 5.23: $K_{\mathrm{I}}, K_{\mathrm{II}}$ und $K_{\mathrm{III}}$ für Fehlerstelle 7 unter Ermüdungslast

In Fig. 5.24 schliesslich werden die SIF $K_{\mathrm{I}, \max }$ und $K_{\mathrm{I}, \text { min }}$ für die Fehlerstelle 8 der Knoten 416 und 434 zusammengefasst. Für den Knoten 416 werden zwei Belastungszustände $L C 1$ und LC2 für die Rissfortschrittssimulation herangezogen (Fig. 5.15), während für den Knoten 434 nur ein Belastungszustand gewählt wird (Fig. 5.17). 


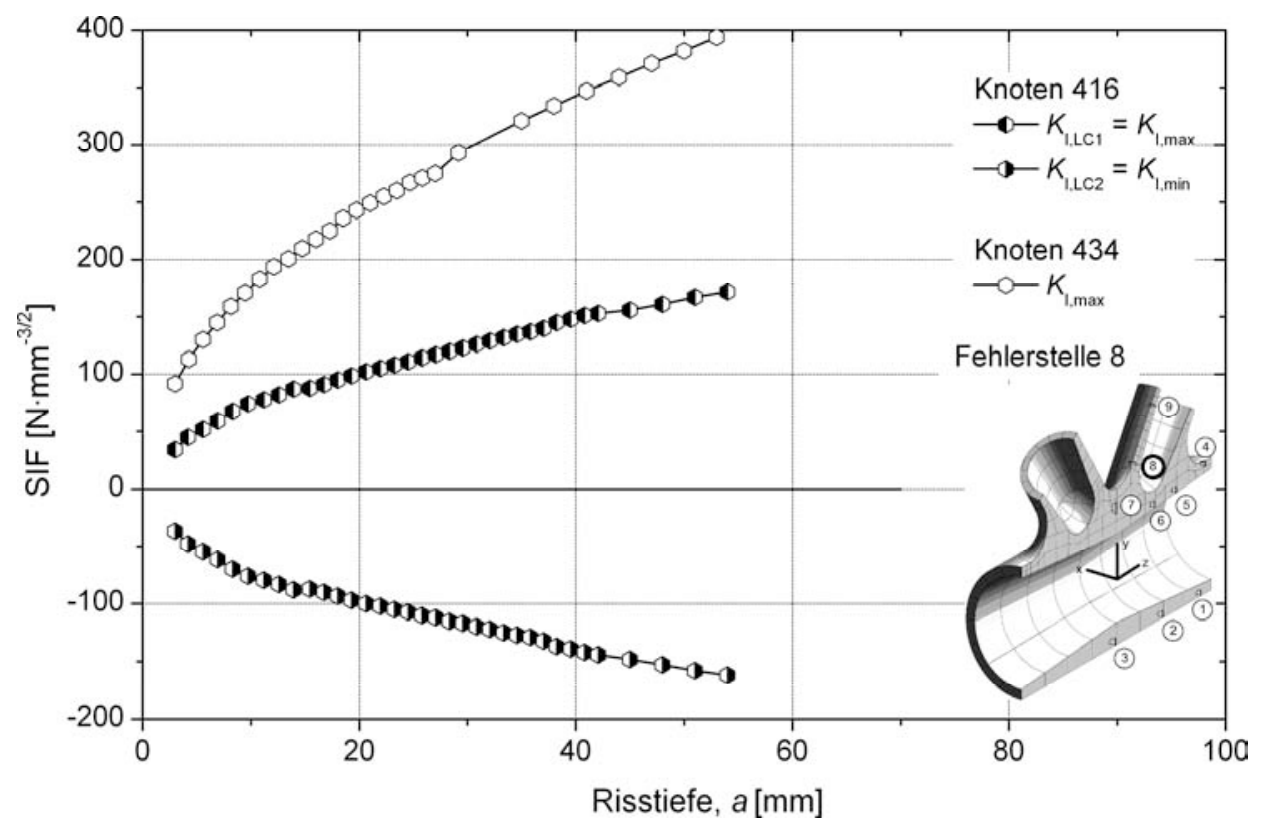

Fig. 5.24: $K_{\mathrm{I}, \max }$ und $K_{\mathrm{I}, \min }$ für Fehlerstelle 8 unter Ermüdungslast

\subsubsection{Ergebnisse der SIF unter statische Belastung (ULS)}

Für die unter der Ermüdungsbelastung berechneten Rissfronten der einzelnen Fehlerstellen wird nun der Spannungsintensitätsfaktor $K_{\text {ULS }}$ unter statischer Last (aus Tab. 5.2) berechnet, um damit den Sprödbruchnachweis (Abschnitt 5.4.2) durchführen zu können. Die Berechnungen zeigen, dass unter statischer Last der Spannungszustand ebenfalls einachsig ist, da $K_{\text {II,ULS }}$ und $K_{\text {III,ULS }}$ annähernd null sind. Die Richtung der maximalen Hauptspannung ist dieselbe wie unter Ermüdungslast. Aus diesem Grund werden in den nachstehenden Figuren nur die SIF $K_{\mathrm{I}, \mathrm{ULS}}$ abgebildet.

In Fig. 5.25 und Fig. 5.26 sind die SIF für die Knoten 416 und 434 dargestellt. Hierbei handelt es sich um die Werte am Rissrand $K_{\mathrm{I}, \mathrm{ULS}}^{\mathrm{c}}$, da diese im Vergleich zu den Werten in der Risstiefe $K_{\mathrm{I}, \mathrm{ULS}}^{\mathrm{a}}$ höher und deshalb für den Sprödbruch ausschlaggebend sind. Da, wie aus Tab. 5.2 ersichtlich ist, für den Knoten 416 die Schnittkräfte an den Diagonalstutzen unter statischer Last niedriger ausfallen als unter der Ermüdungslast (Fig. 5.9), werden für den Sprödbruchnachweis an den Fehlerstellen 8 und 9 die SIF unter Ermüdungslast verwendet.

Fig. 5.25 und Fig. 5.26 zeigen, dass die Spannungsintensitätsfaktoren unter statischer Belastung $K_{\mathrm{I}, \mathrm{ULS}}^{\mathrm{c}}$ weit unter der charakteristischen Bruchzähigkeit des Stahlgusses $K_{\mathrm{Ic}}$ (Abschnitt 3.2.2.2) zu liegen kommen. Unter statischer Belastung ist für den Knoten 416 der Untergurt an den Stutzenenden mit 50 \% der Fliessgrenze und für den Knoten 434 die Diagonale mit $60 \%$ der Fliessgrenze auf Zug beansprucht. Auf Grund des niedrigen Spannungsniveaus im Knoten unter der statischen Belastung steigen die Hauptspannungen im Restquerschnitt aller Stellen zwar an, liegen jedoch weit unter der Fliessgrenze $f_{\mathrm{y}}$.

Für den vorliegenden Anwendungsfall ist demnach die massgebende Fehlergrösse $a_{\text {mass, } i}$ für alle Stellen $i$ nicht durch Sprödbruch sondern durch das Kriterium der maximalen Risstiefe von $90 \%$ der Wanddicke $w_{i}$ gegeben. Tab. 5.3 gibt die Wanddicken bei den untersuchten Fehlerstellen an. Bei den Fehlerstellen 1-4, 8 und 9 ist die maximale Risstiefe gleich $90 \%$ der Wanddicke des Rohrstutzens. Für die Fehlerstelle 5 und 6 werden 90 \% der Wanddicke des Rohrstutzens angesetzt, ohne die zusätzliche geometrisch bedingte Wandverdickung im Knoten zu berücksichtigen. An der Stelle 
7 entspricht die maximale Risstiefe $90 \%$ des Radius der Kugel, deren Mittelpunkt an der Stelle 7 liegt und die Aussenhaut des Knotens berührt.

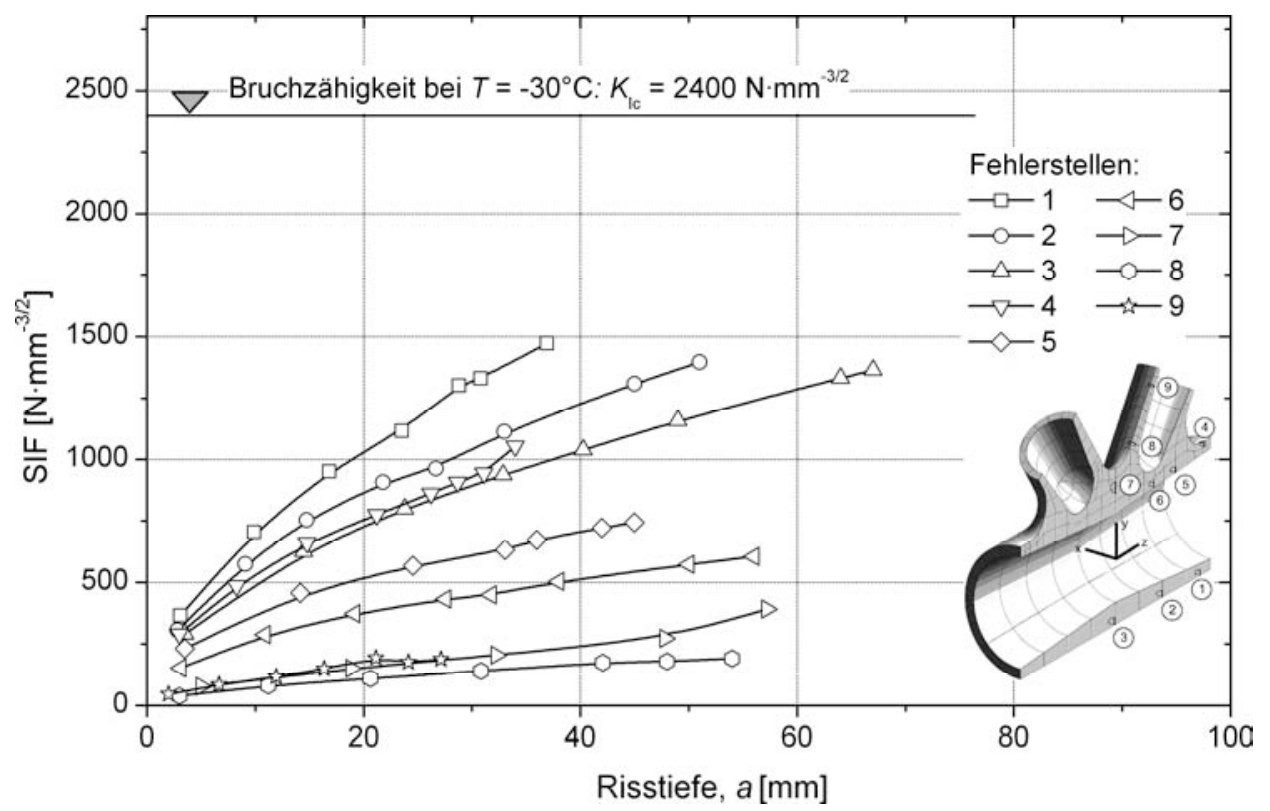

Fig. 5.25: SIF $K_{\mathrm{I}, \mathrm{ULS}}^{\mathrm{c}}$ am Rissrand für alle Fehlerstellen in Knoten 416, unter statischer Last (ULS)

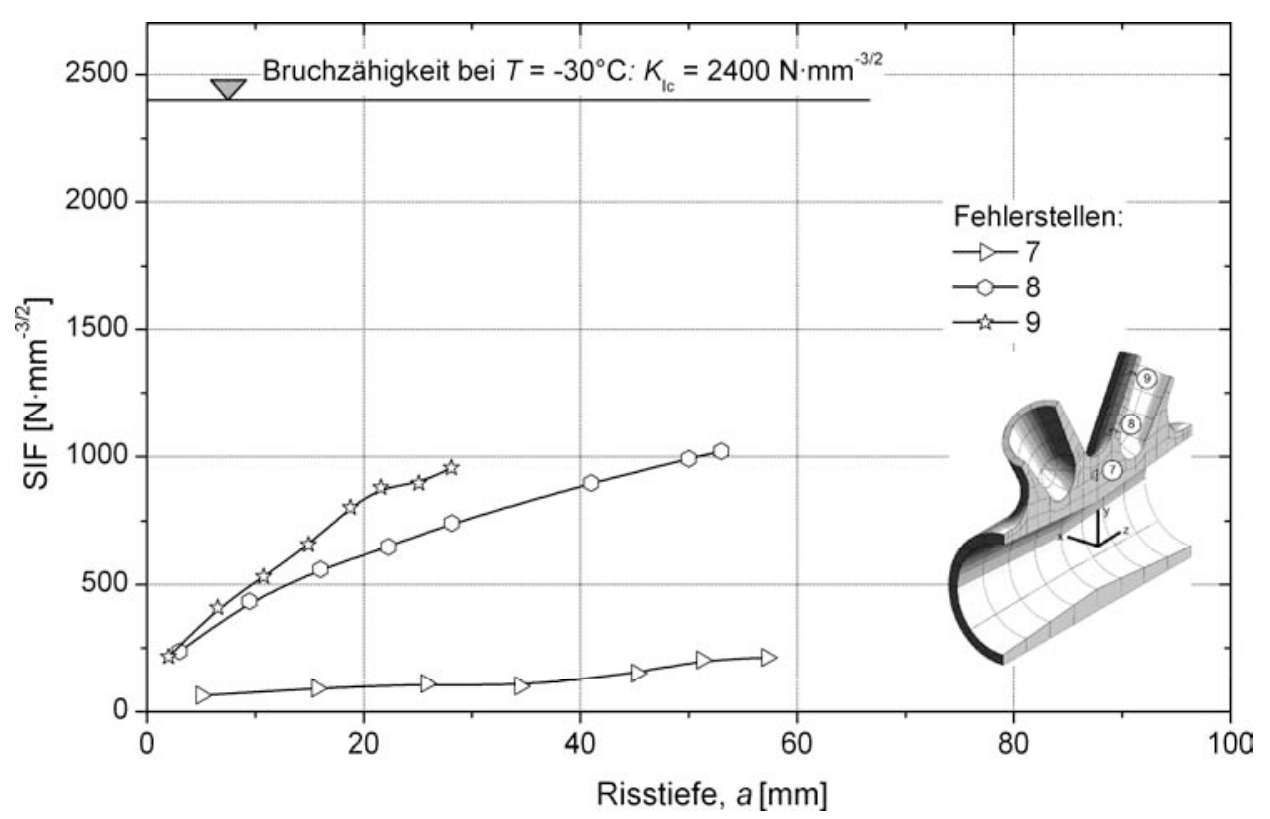

Fig. 5.26: SIF $K_{\mathrm{I}, \mathrm{ULS}}^{\mathrm{c}}$ am Rissrand für alle Fehlerstellen in Knoten 434, unter statischer Last (ULS) 


\subsection{ERGEBNISSE}

Die Ergebnisse zu den maximal zulässigen Anfangsfehlergrössen $a_{0, i}$ sind in Tab. 5.3 zusammengefasst. Die Werte $a_{0, i, \mathrm{BE}}$ wurden anhand der mit BEASY berechneten SIF nach dem in Abschnitt 5.4.2 beschriebenen Vorgehen ermittelt.

\begin{tabular}{cccccc}
\hline & \multicolumn{5}{c}{ Maximal zulässige Anfangsfehlergrösse $a_{0, i}[\mathrm{~mm}]$} \\
\hline Fehlerstelle $i$ & $\begin{array}{c}\text { Wanddicke } w_{i} \\
{[\mathrm{~mm}]}\end{array}$ & \multicolumn{3}{c}{ Knoten 416} & \multicolumn{2}{c}{ Knoten 434} \\
\cline { 3 - 6 } & 41.0 & 11.5 & 28 & - & - \\
\hline 1 & 55.9 & 22.2 & 40 & - & - \\
\hline 2 & 75.0 & 41.7 & 56 & - & - \\
\hline 3 & 38.7 & 14.1 & 36 & - & - \\
\hline 4 & 49.0 & 34.9 & 71 & - & - \\
\hline 5 & 61.9 & 49.8 & 81 & - & - \\
\hline 6 & 63.5 & 55.9 & 88 & 55.2 & 87 \\
\hline 7 & 59.1 & 41.1 & 70 & 37.8 & 64 \\
\hline 8 & 31.6 & 16.1 & 51 & 15.8 & 50 \\
\hline 9 & & & & $a_{0, i} / w_{i}[\%]$ & mit BE \\
\hline
\end{tabular}

Tab. 5.3: Maximal zulässige Anfangsfehlergrössen für die verschiedenen Fehlerstellen im Knoten 416 und Knoten 434

Folgende Beobachtungen können gemacht werden:

- Die maximal zulässigen Anfangsfehlergrössen sind im Verhältnis zur Wanddicke an der jeweiligen Fehlerstelle sehr gross. Die kleinste zulässige Fehlergrösse beträgt $28 \% w_{1}$ und befindet sich an der Fehlerstelle 1.

- Für die Fehlerstelle 7 sind sehr grosse Anfangsfehler - bis zu 88 \% der Wanddicke - zulässig.

- In Tab. 2.2 sind für die Gütestufen 2 bis 4 Gussfehler (= Reflektionsstellen) von maximal $10 \%$ der Wanddicke für die beiden Randzonen und $15 \%$ der Wanddicke für die Kernzone zugelassen. Für die Gütestufe 5 werden für Rand- und Kernzonen 15 bzw. 25 \% der Wanddicke zugelassen. Wenn nun die maximal zulässige Fehlergrösse von $28 \% w_{1}$ an der Fehlerstelle 1 nach [BS 7910:1999] durch zwei sich in den beiden Randzonen befindliche Oberflächenfehler und einen Innenfehler in der Kernzone ersetzt wird, haben die zulässigen Fehler in den Randzonen ungefähr eine Grösse von $10 \%$, in der Kernzone von $17 \% w_{1}$. Die zulässigen Fehlergrössen entsprechen also als den für die Gütestufen 2 bis 4 vorgegebenen.

- Die Anfangsfehlergrössen liegen alle über den in Tab. 2.1 aufgeführten Nachweisgrenzen der Prüfverfahren.

- Für die Diagonalstutzen (Fehlerstellen 7 - 9) sind die Anfangsfehlergrössen des Knotens 434 (nahe beim Auflager) am kleinsten.

\subsection{FOLGERUNGEN}

Für die Untersuchungen zur maximal zulässigen Anfangsfehlergrösse in Stahlgussknoten wird eine für die Kombination von Hohlprofilen und Stahlgussknoten typische Modellfachwerkbrücke entworfen. Daran werden für zwei unterschiedlich belastete Knoten die Beanspruchungen sowohl infolge statischer Last als auch infolge Ermüdungsbelastung bestimmt. Anhand eines numerischen 
Modells mit Randelementen werden Rissfortschrittsimulationen durchgeführt. Folgende Folgerungen können aus den Untersuchungen gezogen werden:

- Das Risswachstum infolge der einmaligen Überfahrt der Ermüdungslast nach der Norm [SIA 261] kann erfasst werden, indem die Einflusslinie des Spannungsintensitätsfaktors (SIF) in einer Fehlerstelle anhand eines Einheitsrisses berechnet wird. So können für jede zu untersuchende Fehlerstelle die zwei für die Differenz der Spannungsintensitätsfaktoren massgebenden Laststellungen der Ermüdungslast bestimmt werden.

- Da die SIF $K_{\mathrm{I}, \text { ULS }}$ aller untersuchten Fehlerstellen trotz der sehr konservativen Annahme der Verkehrslast als Leiteinwirkung für den Sprödbruchnachweis unterhalb der genormten Bruchzähigkeit des Stahlgusses von $K_{\mathrm{Ic}}=2400 \mathrm{~N} \cdot \mathrm{mm}^{-3 / 2}$ liegen und die Hauptspannungen im Knoten $60 \%$ der Fliessgrenze nicht übersteigen, kann Sprödbruch und plastischer Bruch des Restquerschnitts ausgeschlossen werden. Für den vorliegenden Anwendungsfall ist also die massgebende Fehlergrösse $a_{\text {mass }, i}$ an der Stelle $i$ durch $90 \%$ der Wanddicke $w_{i}$ gegeben.

- Es wurde eine Reihe konservativer Annahmen zur Berechnung der maximal zulässigen Anfangsfehlergrössen aufgestellt: Simulation der Gussfehler als Oberflächenriss, Sprödbruchnachweis mit Bruchzähigkeit $K_{\text {Ic }}$ (für ebenen Dehnungszustand) und den Maximalwerten der Spannungsintensitätsfaktoren, Vernachlässigung des Risswachstums nach Wanddurchbruch und Marge von $10 \%$ Wanddicke bevor Wanddurchbruch. Trotz dieser konservativer Annahmen sind die maximal zulässigen Fehlergrössen im Verhältnis zur Wanddicke durchwegs sehr gross. Die Spanne der zulässigen Fehlergrössen reicht von 28 bis 88 \% der Wanddicke. Für die Diagonalstutzen ergeben sich die kleinsten zulässigen Anfangsfehler beim Auflager, für die Untergurtstutzen in Feldmitte der Fachwerkbrücke.

- Wird der kleinste zulässige Oberflächenfehler von $28 \%$ der Wanddicke durch zwei Oberflächenfehler in beiden Randzonen und einen Innenfehler in der Kernzone der Wanddicke ersetzt, liegen deren maximal zulässigen Fehlergrösse mit $10 \%$ bzw.17 \% der Wanddicke im nach der Norm festgelegten Grenzwert für die Gütestufe 4. Die Anfangsfehlergrössen liegen alle über den in Tab. 2.1 für verschiedene Prüfverfahren aufgeführten Nachweisgrenzen. 


\section{VERALLGEMEINERUNG UND PARAMETERSTUDIE}

\subsection{VORGEHEN}

Das Vorgehen zur Untersuchung der allgemeinen Qualitätsanforderungen an Stahlgussknoten im Brückenbau ist in Fig. 6.1 schematisch dargestellt. Die in Kapitel 5 mit dem Programm BEASY berechneten Spannungsintensitätsfaktoren (SIF) in der Risstiefe $K_{\mathrm{I}}^{\mathrm{a}}$ und am Rissrand $K_{\mathrm{I}}^{\mathrm{c}}$ werden durch eine vereinfachte Näherung mit einem konstanten Korrekturfaktor $Y$ ausgedrückt. Die Anwendbarkeit der Näherungsformel wird anhand von Literaturangaben überprüft. Zudem werden die in Kapitel 5 anhand der BEASY Berechnungen ermittelten maximal zulässigen Anfangsfehlergrössen $a_{0, i}$ den mit der Näherungsformel berechneten Werten gegenübergestellt und verglichen.

Die Näherungsformel dient anschliessend zur Durchführung einer Parameterstudie, die den Einfluss von Materialeigenschaften wie Zähigkeit und Fliessgrenze, von verschiedenen Kombinationen aus Ermüdungs- und Verkehrslast sowie der durch den Brückentyp bedingten Knotenabmessungen auf die maximal zulässige Anfangsfehlergrösse untersucht. Die Parameter mit massgebendem Einfluss können so identifiziert und wichtige Folgerungen für die Festlegung der maximal zulässigen Anfangsgrösse von Gussfehlern abgeleitet werden. Zum Abschluss wird das Vorgehen zur Ermüdungsbemessung von Stahlgussknoten zusammengefasst.

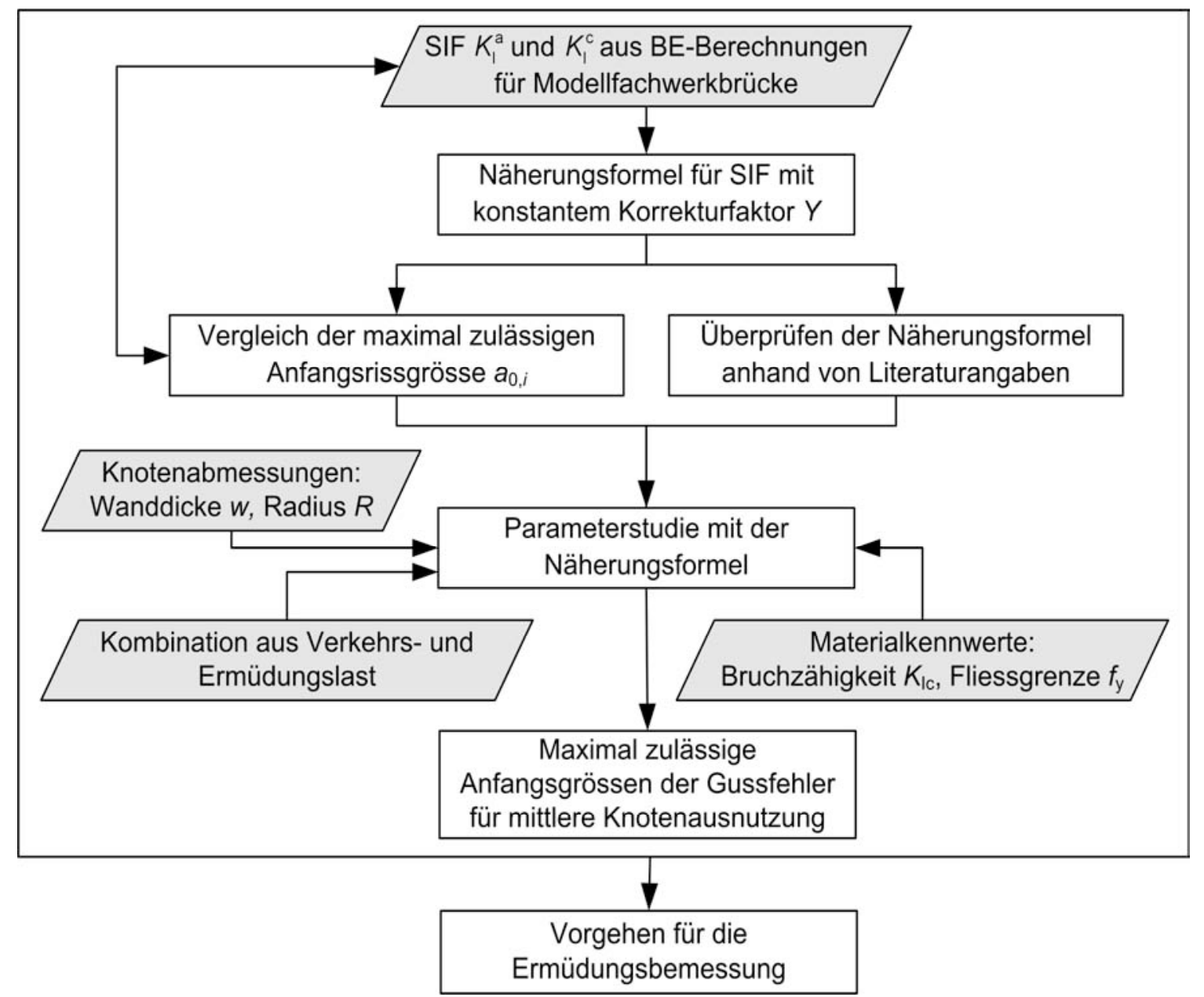

Fig. 6.1: Schematische Darstellung des Vorgehens 


\subsection{NÄHERUNGSFORMEL FÜR DEN SPANNUNGSINTENSITÄTSFAKTOR}

\subsubsection{Herleitung}

Es wird eine Näherungsformel für den Spannungsintensitätsfaktor (SIF) von in Stahlgussknoten vorhanden Gussfehlern gesucht, die eine gute und einfache Abschätzung der maximal zulässigen Anfangsfehlergrössen bei der Ermüdungsbemessung von Stahlgussknoten ermöglicht, ohne dass numerische Risswachstumssimulationen durchgeführt werden müssen.

Die Ergebnisse aus den BE-Berechnungen im Abschnitt 5.4.3 lassen darauf schliessen, dass eine Möglichkeit zur vereinfachten Formulierung des SIF $K_{\mathrm{I}}$ darin bestehen könnte, einen konstanten Korrekturfaktor $Y$ für alle Fehlerstellen im Stahlgussknoten zu definieren. Diese Formulierung bietet den Vorteil, dass die Integration der Paris-Erdogan Gleichung zur Ermittlung der zulässigen Anfangsgrösse der Gussfehler wesentlich vereinfacht wird. Der konstante Korrekturfaktor geht wie folgt in die Formulierung des SIF ein:

$$
K_{\mathrm{I}}=Y \cdot \sigma_{1} \cdot \sqrt{\pi \cdot a}
$$

$K_{\mathrm{I}} \quad$ Spannungsintensitätsfaktor

$\sigma_{1} \quad$ erste Hauptzugspannung an der Fehlerstelle (unter Berücksichtigung ev. Spannungskonzentration)

a Risstiefe

Y konstanter Korrekturfaktor

Folgende Annahmen werden bei der Einführung eines konstanten Korrekturfaktors gemacht:

- Es wird zwischen Oberflächenrissen und Innenrissen unterschieden.

- Wenn die Risstiefe $a$ die Wanddicke $w$ erreicht und es zum Durchbruch kommt, nimmt der SIF in der Risstiefe stark zu. Durch die Annahme eines konstanten Korrekturfaktors wird das Risswachstum daher in dieser Phase unterschätzt. Der Anteil des Risswachstums von $a=0.9 \cdot w$ bis $a=w$ an der gesamten Lebensdauer beträgt generell nur wenige Prozente. Aus diesem Grund ist die Näherung zulässig, das Risswachstum bis zu einer Risstiefe von 90 \% der Wanddicke mit einem konstanten Korrekturfaktor $\mathrm{zu}$ beschreiben und den Anteil des Risswachstum für 0.9. $w \leq a<w$ zu vernachlässigen. Die $10 \%$ der Wanddicke können auch als Sicherheitsmarge betrachtet werden.

- Das Verhältnis a / $c$ wird konstant angenommen. BE-Berechnungen im Abschnitt 5.4.3 bestätigen diese Annahme, da das Verhältnis vom anfänglich angesetzten Verhältnis von 1.0 (siehe Abschnitt 5.4.3.2) während der Risswachstumssimulation nur auf durchschnittlich 0.9 abfällt.

In [Barsom et al. 1999] wird für einen kreisförmigen Innenriss in einem unendlichen Halbraum ebenfalls ein konstanter Korrekturfaktor angegeben. Der Spannungsintensitätsfaktor wird wie folgt formuliert:

$$
K_{\mathrm{I}}=\frac{2}{\sqrt{\pi}} \cdot \sigma_{1} \cdot \sqrt{a}=0.64 \cdot \sigma_{1} \cdot \sqrt{\pi \cdot a}
$$

Der konstante Korrekturfaktor $Y=0.64$ für einen kreisrunden Innenriss wird durch die Auswertung der Ergebnisse aus den BE-Berechnungen bestätigt (vergleiche Tab. 6.1).

Zur Berechnung des Korrekturfaktors werden die SIF der verschiedenen Fehlerstellen zunächst normiert, indem sie durch die erste Hauptzugspannung an der Fehlerstelle (siehe Abschnitt 6.4) di- 
vidiert werden. Die Normierung erlaubt einen direkten Vergleich und die Auswertung der SIF an den verschiedenen Fehlerstellen. Daraus ergibt sich folgender normierter SIF:

$$
\hat{K}_{\mathrm{I}}=Y \cdot \hat{\sigma}_{1} \cdot \sqrt{\pi \cdot a}
$$

$\hat{K}_{\mathrm{I}} \quad$ normierter Spannungsintensitätsfaktor

$\hat{\sigma}_{1} \quad$ Einheitsspannung in Richtung der ersten Hauptspannung, $\hat{\sigma}_{1}=1 \mathrm{MPa}$

a Risstiefe

Y konstanter Korrekturfaktor

Die untersuchten Fehlerstellen 1- 6, 8 und 9 (siehe Fig. 5.11 auf Seite 86) sind Oberflächenrisse, die Fehlerstelle 7 hingegen ist ein Innenriss und wird daher für die Bestimmung des Korrekturfaktors getrennt betrachtet.

Die mit dem Programm BEASY unter der Ermüdungsbelastung berechneten SIF in der Risstiefe $K_{\mathrm{I}}^{\mathrm{a}}$ und am Rissrand $K_{\mathrm{I}}^{\mathrm{c}}$ werden über die an der Fehlerstelle herrschenden ersten Hauptzugspannungen normiert. Die normierten SIF für die Oberflächenrisse werden in Fig. 6.2 und Fig. 6.3, diejenigen für die Innenrisse in Fig. 6.4 und Fig. 6.5 dargestellt. Die Abszisse zeigt jeweils die Wurzel der Risstiefe, die Ordinate die SIF.

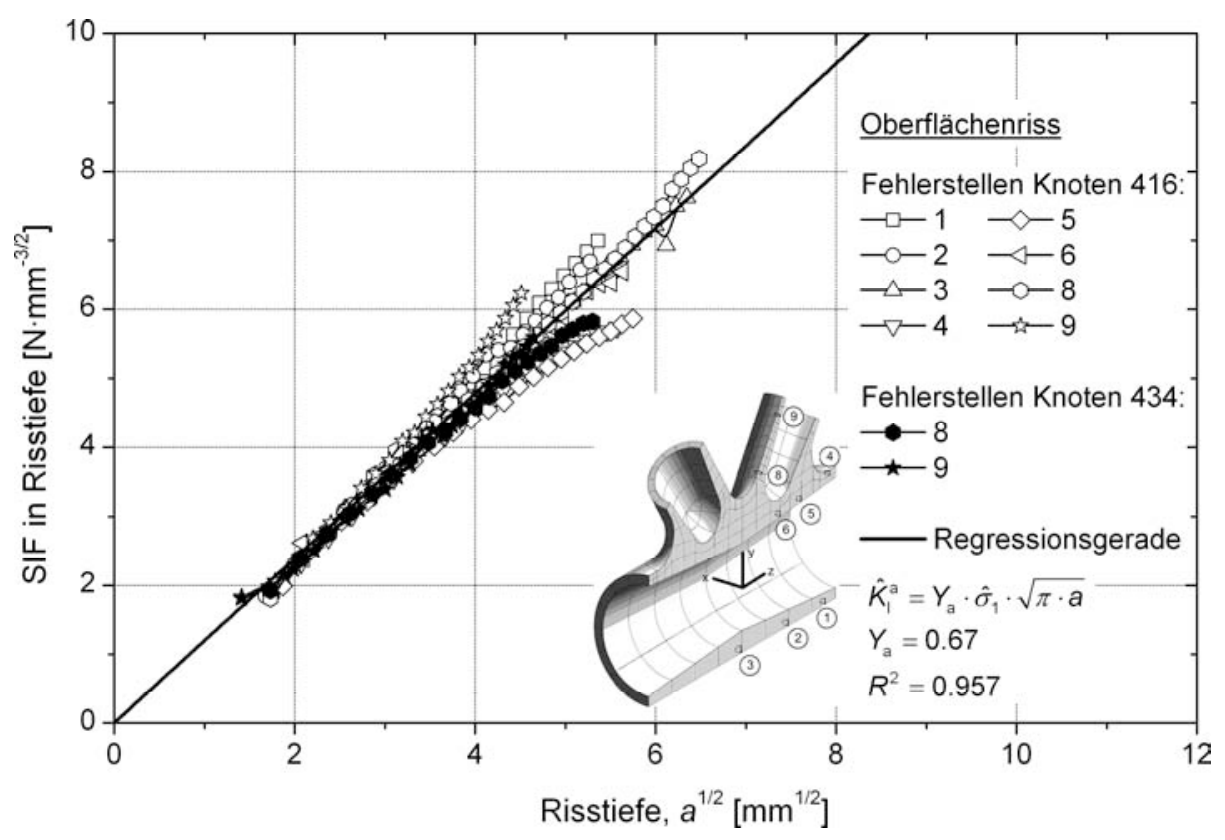

Fig. 6.2: SIF in der Risstiefe $\hat{K}_{\mathrm{I}}^{\mathrm{a}}$ für Oberflächenrisse 


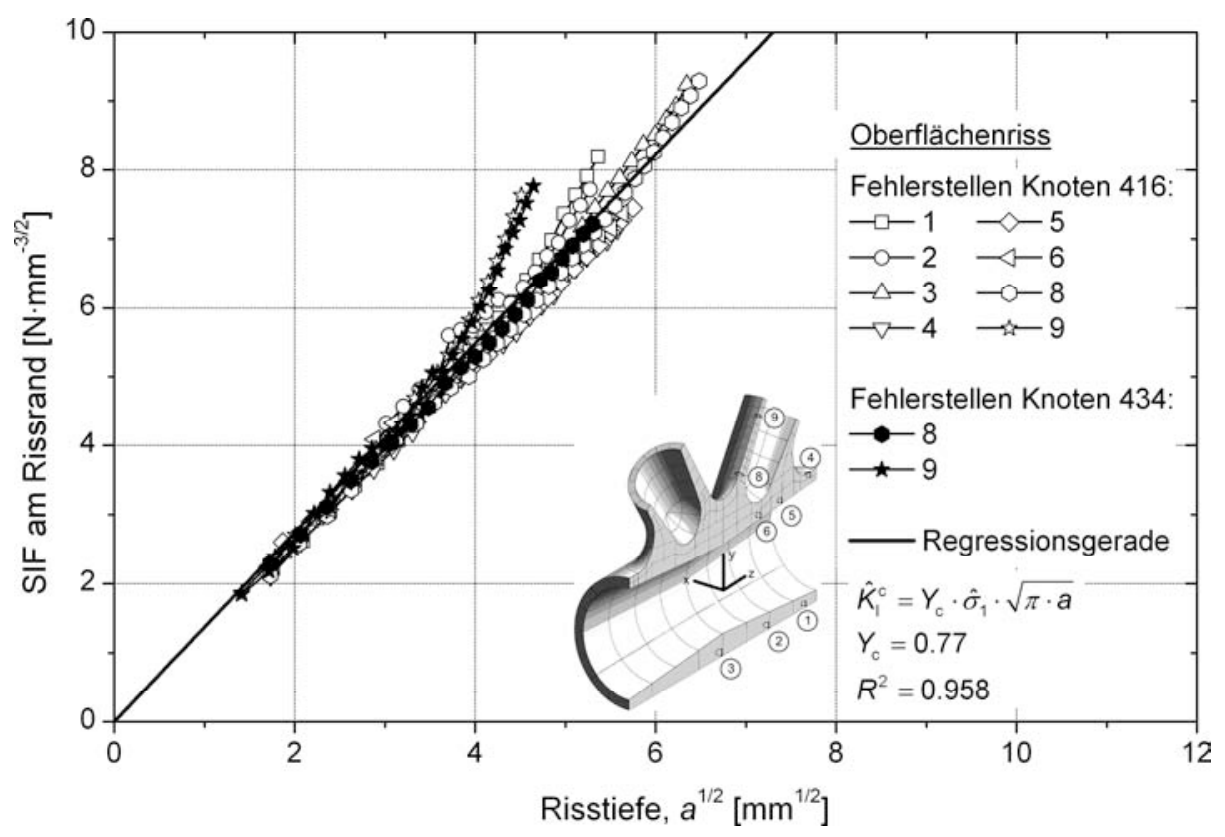

Fig. 6.3: SIF am Rissrand $\hat{K}_{\mathrm{I}}^{\mathrm{c}}$ für Oberflächenrisse

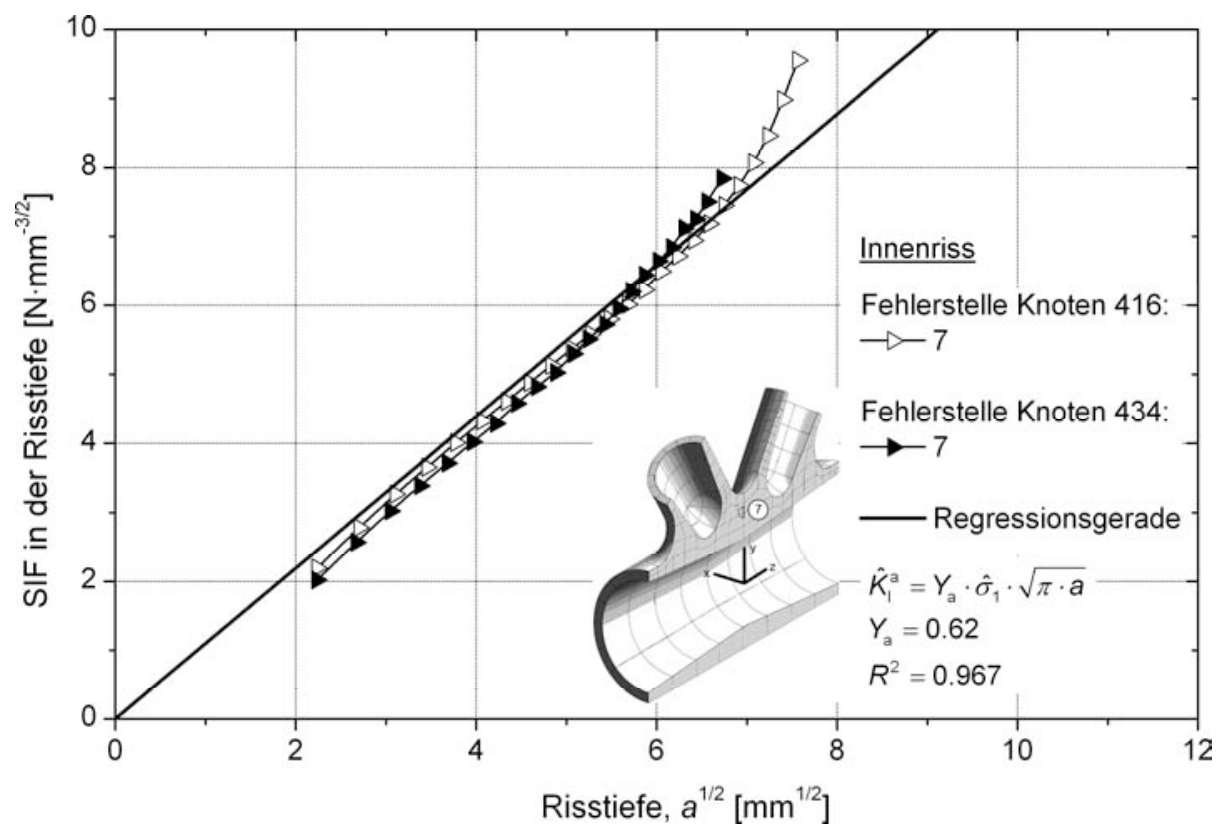

Fig. 6.4: SIF in der Risstiefe $\hat{K}_{\mathrm{I}}^{\mathrm{a}}$ für Innenrisse 


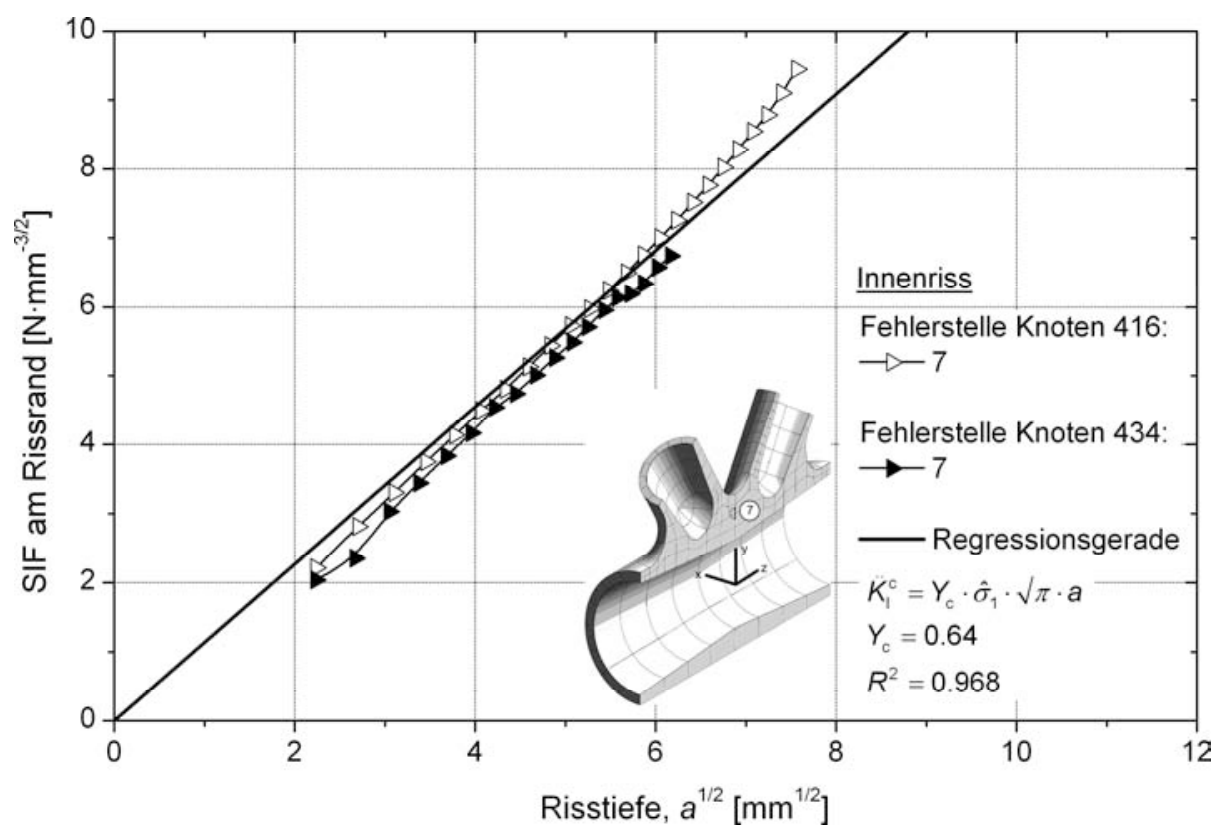

Fig. 6.5: SIF am Rissrand $\hat{K}_{\mathrm{I}}^{\mathrm{c}}$ für Innenrisse

Sowohl unter Ermüdungslast als auch unter statischer Last ist der Spannungszustand in der Risstiefe einachsig und die Richtung der maximalen Hauptspannung dieselbe. Die Fehlerstelle 7 ist eine Ausnahme, da der Ermüdungsriss nicht genau senkrecht zur ersten Hauptspannung unter statischer Last liegt. Für die Berechnung der konstanten Korrekturfaktoren werden trotz dieser Ausnahme die normierten SIF unter Ermüdungsbelastung für die Berechnung benutzt.

Die Ergebnisse zeigen, dass für die Oberflächenrisse nicht nach der Lage auf der Aussenseite (Fehlerstellen 1 - 4) oder auf der Innenseite (5 und 6) des Knotens unterschieden werden muss, da die Werte sich nicht wesentlich von einander unterschieden. Diese Feststellung wird auch in [Bergmann 1995] für Oberflächenrisse auf der Aussen- und Innenseite von Rohren gemacht.

Aus der Annahme eines konstanten Korrekturfaktors $Y$ folgt ein linearer Zusammenhang zwischen $\hat{K}_{\mathrm{I}}^{\mathrm{a}}$ bzw. $\hat{K}_{\mathrm{I}}^{\mathrm{c}}$ und $a^{1 / 2}$, der mit folgender Regressionsgerade beschrieben wird:

$$
y=m \cdot x+b
$$

$x, y \quad$ Koordinaten auf der Abszisse bzw. auf der Ordinate $\left(x=a^{1 / 2}, y=\hat{K}_{\mathrm{I}}\right)$

$m \quad 1$. Regressionskoeffizient (Steigung der Regressionsgerade)

$b \quad 2$. Regressionskoeffizient (Ordinatenabschnitt)

Da bei einer Risstiefe $a=0 \mathrm{~mm}$ die SIF den Wert $K_{\mathrm{I}}^{\mathrm{a}}=K_{\mathrm{I}}^{\mathrm{c}}=0 \mathrm{~N} \cdot \mathrm{mm}^{-3 / 2}$ annehmen, muss die Regressionsgerade durch den Nullpunkt gehen. Somit gilt:

$$
\begin{gathered}
m=Y \cdot \hat{\sigma}_{1} \cdot \sqrt{\pi} \\
b=0
\end{gathered}
$$

Der Determinationskoeffizient $R^{2}$ ist ein Mass für die Abweichung eines Regressionsmodells von den empirischen Daten. Gelegentlich wird auch die Bezeichnung Bestimmtheitsmass verwendet. $R^{2}$ nimmt Werte zwischen 0 und 1 an. $R^{2}=0$ bedeutet, dass das Modell keine Vorhersage der numerisch ermittelten Werte erlaubt, $R^{2}=1.0$ weist auf eine perfekte Übereinstimmung zwischen Modell und numerisch ermittelten Werten hin. Der Determinationskoeffizient wird wie folgt berechnet: 


$$
R^{2}=1-\frac{\sum_{i=1}^{n}\left(y_{\mathrm{i}}-\hat{y}_{\mathrm{i}}\right)^{2}}{\sum_{i=1}^{n} y_{\mathrm{i}}^{2}-\frac{1}{n}\left(\sum_{i=1}^{n} y_{\mathrm{i}}\right)^{2}}
$$

$y_{\mathrm{i}} \quad$ numerisch ermittelte Ordinatenwerte

$\hat{y}_{\mathrm{i}} \quad$ Ordinatenwerte aus der Regressionsgerade: $\hat{y}_{\mathrm{i}}=m \cdot x_{\mathrm{i}}$

$n \quad$ Anzahl numerisch ermittelter Werte

Tab. 6.1 vergleicht die Korrekturfaktoren mit den entsprechenden Determinationskoeffizienten für Oberflächen- und Innenrisse an den untersuchten Fehlerstellen der Stahlgussknoten aus der Modellfachwerkbrücke (Fig. 5.11 auf Seite 86). Die Determinationskoeffizienten liegen durchwegs sehr nahe bei 1.0, was eine sehr kleine Abweichung der Vorhersage mittels Regressionsmodell von den numerisch ermittelten Werten bedeutet.

\begin{tabular}{ccccc}
\hline & \multicolumn{2}{c}{ Risstiefe } & \multicolumn{2}{c}{ Rissrand } \\
\hline Kategorie & $Y_{\mathrm{a}}$ & $R^{2}$ & $Y_{\mathrm{c}}$ & $R^{2}$ \\
\hline Oberflächenriss & 0.67 & 0.957 & 0.77 & 0.958 \\
\hline Innenriss & 0.62 & 0.967 & 0.64 & 0.968 \\
\hline
\end{tabular}

Tab. 6.1: Korrekturfaktoren für Oberflächen- und Innenrisse aus der Regression

Für den Oberflächenriss unterscheiden sich die Korrekturfaktoren in der Risstiefe $Y_{\mathrm{a}}=0.67$ und am Rissrand $Y_{\mathrm{c}}=0.77$. Dies bedeutet, dass der Riss in die Länge etwas schneller wächst als in die Tiefe. Für den Innenriss sind die Korrekturfaktoren $Y_{\mathrm{a}}=0.62$ und $Y_{\mathrm{c}}=0.64$ fast identisch, was für einen kreisrunden Innenriss charakteristisch ist. Statistisch sind diese beiden Werte kaum unterscheidbar. Daher wird für den Innenriss ein einheitlicher Wert $Y=0.64$ angesetzt.

\subsubsection{Literaturvergleich}

Inwiefern eine Näherungsformel für den Spannungsintensitätsfaktor mit einem konstanten Korrekturfaktor vertretbar ist, soll zusätzlich anhand von Literaturquellen überprüft werden. Der Vergleich wird anhand eines Oberflächenrisses mit einer Rissform $a / c=1.0$ auf der Innenseite eines Rundhohlprofils angestellt. Die Ergebnisse aus den Literaturquellen werden mit BE-Berechnungen verglichen.

In [BS 7910:1999] werden für die Rissform $a / c=1.0$, ein Verhältnis der Wanddicke zum Innenradius $w / R_{\mathrm{i}}=0.1\left(w=10 \mathrm{~mm}\right.$ und $\left.R_{\mathrm{i}}=123.5 \mathrm{~mm}\right)$ und einige Risstiefen $a / w=0.0,0.2,0.4,0.6,0.8$ Korrekturfaktoren für die Risstiefe $Y_{\mathrm{a}}$ bzw. für den Rissrand $Y_{\mathrm{c}}$ tabellarisch aufgeführt.

In [Bergmann 1995] wird für den Korrekturfaktor eine Parametergleichung aufgestellt. Diese Gleichung beschreibt für jede Position entlang einer Rissfront in Abhängigkeit der Risstiefe $a$ / $w=0.2$, 0.4, 0.6, 0.8 und des Verhältnisses der Wanddicke zum Innenradius $w / R_{\mathrm{i}}=0.1$ den Korrekturfaktor. Die Parameter sind tabellarisch zusammengestellt.

[Zahoor 1985] gibt für den SIF in der Risstiefe folgende analytische Gleichung:

$$
K_{\mathrm{I}}=\sigma_{1} \sqrt{\frac{\pi \cdot a}{Q} \cdot F_{\mathrm{t}}}
$$


mit

$$
\begin{gathered}
F_{\mathrm{t}}=1+\left[0.02+\xi \cdot(0.0103+0.00617 \cdot \xi)+0.0035 \cdot(1+0.7 \cdot \xi) \cdot\left(\frac{R_{\mathrm{m}}}{w}-5\right)^{0.7}\right] \cdot Q^{2} \\
\xi=\frac{2 \cdot c}{w} \quad Q=1+1.464 \cdot\left(\frac{a}{c}\right)^{1.65}
\end{gathered}
$$

a Risstiefe

c Rissbreite

$w \quad$ Wanddicke

$R_{\mathrm{m}} \quad$ mittlerer Radius

Als weiteren Vergleich werden die analytische Gleichungen in [Newmann et al. 1986] zur Berechnung der SIF eines Oberflächenrisses in einer Platte angegeben. Die Plattenbreite $b$ wird hier gleich dem Rohrumfang angenommen. Unter Zugspannung

$$
\begin{gathered}
K_{\mathrm{I}}=\sigma_{1} \cdot\left(\frac{\pi \cdot a}{Q}\right)^{1 / 2} \cdot F_{\mathrm{s}} \\
F_{\mathrm{s}}=\left[M_{1}+M_{2} \cdot\left(\frac{a}{w}\right)^{2}+M_{3} \cdot\left(\frac{a}{w}\right)^{4}\right] \cdot g \cdot f_{\Phi} \cdot f_{\mathrm{w}} \\
M_{1}=1.13+0.09 \frac{a}{c} \quad M_{2}=-0.54+\frac{0.89}{0.2+a / c} \quad M_{3}=0.5-\frac{1}{0.65+a / c}+14 \cdot(1-a / c)^{24} \\
g=1+\left[0.1+0.35\left(\frac{a}{w}\right)^{2}\right](1-\sin \Phi)^{2} \\
f_{\Phi}=\left[\left(\frac{a}{c}\right)^{2} \cos ^{2} \Phi+\sin ^{2} \Phi\right]^{1 / 4} \quad f_{\mathrm{w}}=\left[\sec \left(\frac{\pi c}{2 b}\right) \cdot \sqrt{\frac{a}{w}}\right]^{1 / 2}
\end{gathered}
$$

$\Phi \quad$ Winkel, mit dem jede Position entlang der Rissfront beschrieben wird $(0 \leq \Phi \leq \pi)$

$b \quad$ Plattenlänge, Annahme $b=2 \pi \cdot R_{\mathrm{m}}$

Fig. 6.6 vergleicht die normierten SIF $\hat{K}_{\mathrm{I}}^{\mathrm{a}}$ und $\hat{K}_{\mathrm{I}}^{\mathrm{c}}$ (mit $\hat{\sigma}_{1}=1 \mathrm{MPa}$ ) aus den BE-Berechnungen und aus den Literaturangaben für den Oberflächenriss im Rundhohlprofil. Der Vergleich zeigt eine sehr gute Übereinstimmung zwischen den Ergebnissen nach [Bergmann 1995], nach [Newmann et al. 1986] und den BE-Berechnungen. Die Angaben nach [BS 7910:1999] stimmen zwar für die SIF in der Risstiefe ebenfalls gut überein, die Werte am Rissrand weichen jedoch von den BEBerechnungen und den Berechnungen nach [Bergmann 1995] und [Newmann et al. 1986] ab. Da die Ergebnisse nach [Zahoor 1985] für die SIF in der Risstiefe sehr stark von den anderen Werten abweichen, werden diese nicht betrachtet.

Die Regressionsgeraden der SIF nach [Bergmann 1995] zeigen, dass der Korrekturfaktor tatsächlich bis zu einer Risstiefe $a$ / $w=0.8$ als konstant angesehen werden kann. Im vorliegenden Fall betragen die Korrekturfaktoren $Y_{\mathrm{a}}=0.69$ und $Y_{\mathrm{c}}=0.83$. Die Korrekturfaktoren in der Risstiefe sind gleich gross. $Y_{\mathrm{c}}$ nach [Bergmann 1995] ist etwas höher. Dies liegt daran, dass das Verhältnis $a / c=1.0$ während des Risswachstums konstant angenommen wurde. Im Verlaufe der Risswachstumssimulation im Stahlgussknoten ändert sich die Rissform leicht und pendelt sich auf durchschnittlich $a / c=0.9$ ein. Durch diese Rissformanpassung sind die SIF am Rissrand niedriger. 
Die aus den BE-Berechnungen ersichtliche starke Zunahme des SIF, sobald sich die Risstiefe der Wanddicke nähert, wurde in Abschnitt 6.2.1 bereits diskutiert.

Die Wahl einer Näherungsformel für den Spannungsintensitätsfaktor mit einem konstanten Korrekturfaktor ermöglicht also eine gute und einfache Abschätzung der maximal zulässigen Anfangsfehlergrössen bei der Bemessung von Stahlgussknoten.

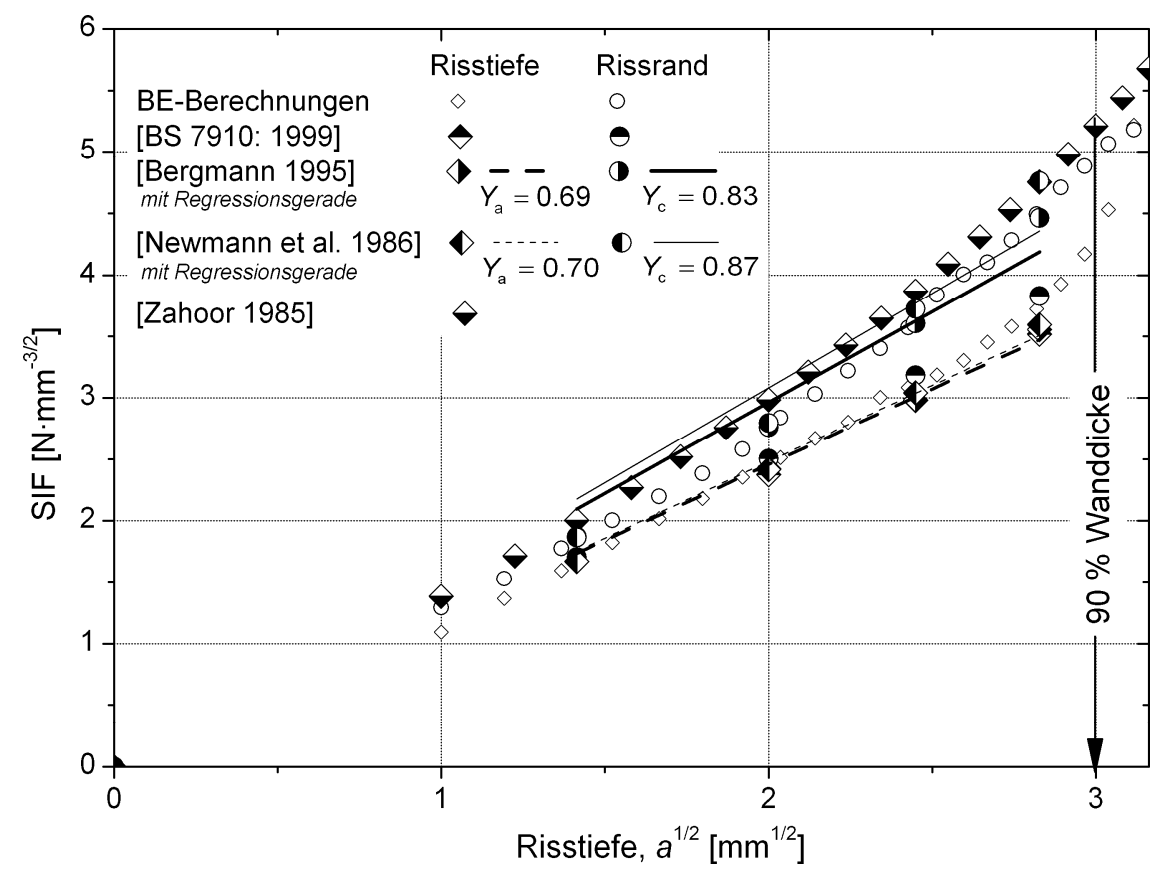

Fig. 6.6: SIF $\hat{K}_{\mathrm{I}}^{\mathrm{a}}$ und $\hat{K}_{\mathrm{I}}^{\mathrm{c}}$ für Oberflächenrisse im Rundhohlprofil

\subsection{3 Überprüfung anhand der Modellfachwerkbrücke}

Die in Tab. 6.1 zusammengestellten Korrekturfaktoren werden für die Berechnung der maximal zulässigen Anfangsfehlergrösse in den Fehlerstellen der Knoten 416 und 434 (Fig. 5.11 auf Seite 86) benutzt. Da eine Abweichung des Korrekturfaktors $Y$ gemäss der Gleichung (5.8) in der dritten Potenz in die Berechnung des Risswachstums eingeht und im Integral dieser Fehler noch aufsummiert wird, ist es von grossem Interesse zu untersuchen, welchen Einfluss die Näherung mit einem konstantem Korrekturfaktor auf die maximal zulässigen Anfangsfehlergrössen hat. In Tab. 6.2 und Fig. 6.7 werden die mit der Näherungsformel berechneten Fehlergrössen denjenigen aus den BEBerechnungen gegenübergestellt. 


\begin{tabular}{|c|c|c|c|c|c|c|c|c|}
\hline & \multirow{3}{*}{$\begin{array}{l}\text { Fehler- } \\
\text { stelle } i\end{array}$} & \multirow{3}{*}{$\begin{array}{c}\text { Wand- } \\
\text { dicke } w_{i} \\
{[\mathrm{~mm}]}\end{array}$} & \multicolumn{6}{|c|}{ Maximal zulässige Anfangsfehlergrösse $a_{0, i}[\mathrm{~mm}]$} \\
\hline & & & \multicolumn{3}{|c|}{ Knoten 416} & \multicolumn{3}{|c|}{ Knoten 434} \\
\hline & & & mit BE & mit $Y$ & $\begin{array}{c}\text { Abwei- } \\
\text { chung [\%] }\end{array}$ & mit BE & mit $Y$ & $\begin{array}{c}\text { Abwei- } \\
\text { chung [\%] }\end{array}$ \\
\hline \multirow{8}{*}{ Oberflächenriss } & 1 & 41.0 & 11.5 & 13.1 & +13.9 & - & - & - \\
\hline & 2 & 55.9 & 22.2 & 25.1 & +13.1 & - & - & - \\
\hline & 3 & 75.9 & 41.7 & 41.6 & -0.3 & - & - & - \\
\hline & 4 & 38.7 & 14.1 & 13.7 & -2.8 & - & - & - \\
\hline & 5 & 49.0 & 34.9 & 33.1 & -5.2 & - & - & - \\
\hline & 6 & 61.9 & 49.8 & 50.7 & +1.8 & - & - & - \\
\hline & 8 & 59.1 & 41.1 & 41.6 & +1.2 & 37.8 & 33.5 & -11.4 \\
\hline & 9 & 31.6 & 16.1 & 19.7 & +22.4 & 15.8 & 13.9 & -12.0 \\
\hline Innenriss & 7 & 63.5 & 55.9 & 56.0 & +0.8 & 55.2 & 55.4 & +1.4 \\
\hline
\end{tabular}

Tab. 6.2: Maximal zulässige Anfangsfehlergrössen gemäss BE-Berechnungen und Näherung

Die Abweichungen liegen im Allgemeinen deutlich unter $\pm 20 \%$. Da der konstante Korrekturfaktor $Y$ einen Mittelwert der BE-Berechnungen darstellt, können die Abweichungen sowohl positiv als auch negativ sein. Für den Innenriss sind die Abweichungen sehr gering.

In Anbetracht der übrigen Unsicherheiten, insbesondere auf der Einwirkungsseite, und der wesentlichen Vereinfachung durch die Annahme eines konstanten Korrekturfaktors $Y$ für alle Fehlerstellen im Knoten sind diese Abweichungen akzeptierbar. Mit dem vorgeschlagenen, sehr einfachen Verfahren ist eine relativ genaue Abschätzung der zulässigen Anfangsfehlergrösse möglich.

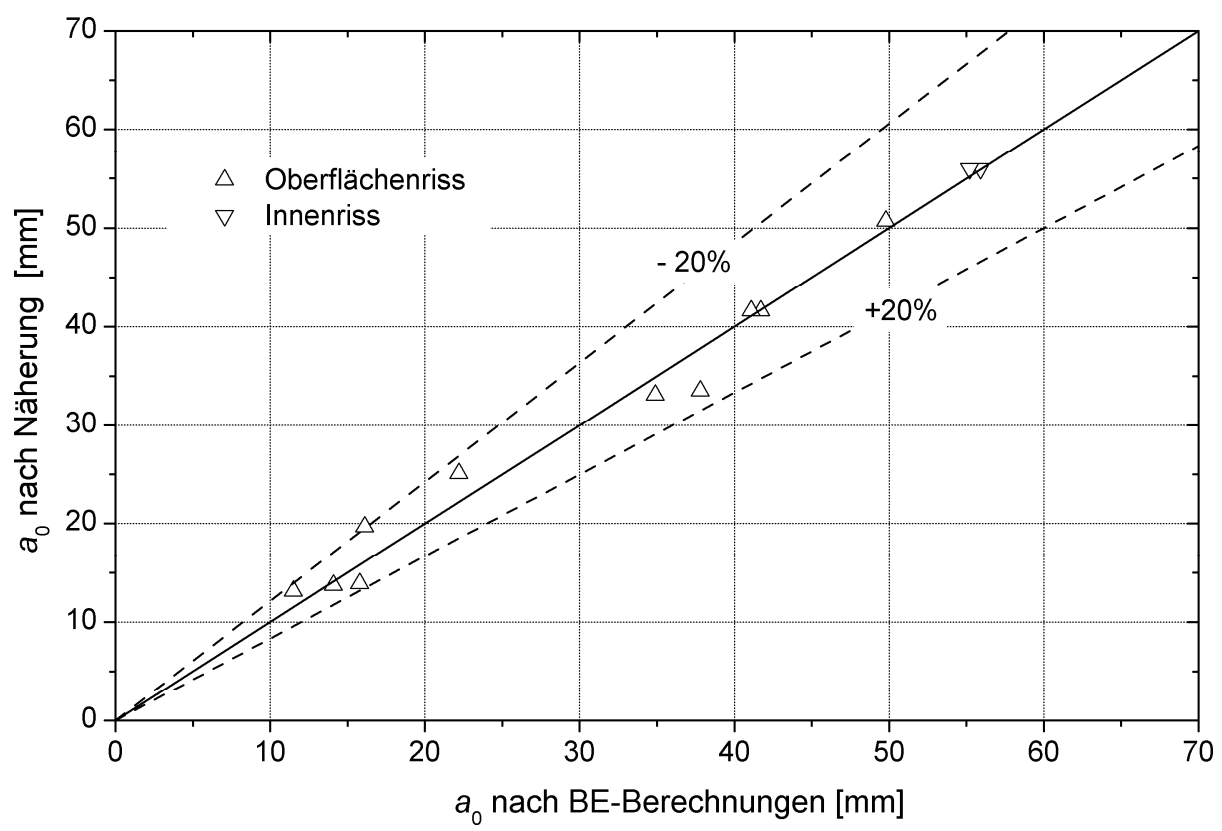

Fig. 6.7: Abweichung der zulässigen Anfangsfehlergrössen gemäss Näherungsformel von denjenigen aus BE-Berechnungen 


\subsection{PARAMETERSTUDIE}

\subsubsection{Rahmen}

Die für die vereinfachte Formulierung des SIF in Tab. 6.1 zusammengefassten konstanten Korrekturfaktoren gelten zunächst nur für die zu ihrer Herleitung benutzte Knotengeometrie. Um die Anwendbarkeit für andere Fälle zu beurteilen, muss der Einfluss der Knotengeometrie auf den Korrekturfaktor quantifiziert werden. Im Abschnitt 6.2 wird gezeigt, dass sich die zulässigen Anfangsfehlergrössen auf die erste Hauptzugspannung an der Fehlerstelle zurückführen lassen. Es kann daher angenommen werden, dass auch bei Knoten mit abweichender Geometrie (z. B. KK-Knoten für einen räumlichen Fachwerkträger) die Hauptspannungen an der Fehlerstelle massgebend sind. Aus diesem Grund wird im Rahmen dieser Arbeit auf Untersuchungen an weiteren Knotengeometrien verzichtet.

Die Parameterstudie beschränkt sich auf die Stutzen der Stahlgussknoten, wobei die Innenrisse also die Fehler im Bereich zwischen den Diagonalstutzen - nicht betrachtet werden, da hier im Vergleich zu den übrigen Bereichen sehr grosse Anfangsfehlergrössen zugelassen sind (Abschnitt 5.5). Demnach befindet sich jede Fehlerstelle in einem Kreisquerschnitt mit einem Aussendurchmesser $D$ und einer Wanddicke $w$. Es wird angenommen, dass der Fehler ein Oberflächenriss ist und senkrecht zur ersten Hauptspannungsrichtung orientiert ist, und dass diese für Verkehrs- und Ermüdungslast nach der Norm [SIA 261] identisch ist.

Für die Berechnung des Risswachstums eines Oberflächenrisses wird der Korrekturfaktor für die Risstiefe $Y_{\mathrm{a}}$ herangezogen. Für den Sprödbruchnachweis wird hingegen $Y_{\mathrm{c}}$ verwendet, da dieser über $Y_{\mathrm{a}}$ liegt, $K_{\mathrm{I}}^{\mathrm{c}}$ also grösser ist als $K_{\mathrm{I}}^{\mathrm{a}}$. Es handelt sich dabei um eine konservative Annahme, da $K_{\mathrm{I}}^{c}$ mit der Bruchzähigkeit für einen ebenen Dehnungszustand $K_{\mathrm{Ic}}$ verglichen wird. Am Rissrand herrscht jedoch ein ebener Spannungszustand, die Bruchzähigkeit liegt entsprechend über $K_{\text {Ic }}$ (vergleiche Tab. 6.1 und Abschnitt 5.4.2).

Die zulässige Anfangsfehlergrösse $a_{0}$ hängt folgendermassen von den Parametern, die die Geometrie, die Materialeigenschaften und den Belastungszustand umschreiben, ab:

$$
\begin{gathered}
a_{0}=a_{0}\left(a_{\text {mass }} ; Y_{a} ; \Delta \sigma_{1, \mathrm{E} 2}\right) \\
a_{\text {mass }}=a_{\text {mass }}\left(w ; f_{\mathrm{y}} ; K_{\mathrm{Ic}} ; Y_{c} ; \gamma ; \sigma_{1, \mathrm{Ed}}\right)
\end{gathered}
$$

$a_{\text {mass }} \quad$ massgebende Fehlergrösse an der Fehlerstelle: $a_{\text {mass }}=\min \left(0.9 w ; a_{\text {krit }}\right)$.

w Wanddicke an der Fehlerstelle

$a_{\text {krit }} \quad$ im Failure Assessment Diagramm ermittelte kritische Fehlergrösse für Sprödbruch (Abschnitt 5.4.2)

$f_{\mathrm{y}} \quad$ rechnerische Fliessgrenze des gewählten Stahlgusses

$K_{\text {Ic }} \quad$ rechnerische Bruchzähigkeit des gewählten Stahlgusses bei $-30^{\circ} \mathrm{C}$

$\gamma \quad$ geometrischer Parameter: $\gamma=D / 2 w$

$D \quad$ äusserer Durchmesser des Knotenstutzens an der Fehlerstelle

$Y_{\mathrm{a}} \quad$ konstanter Korrekturfaktor für die Risstiefe $a$ (Tab. 6.1)

$Y_{c} \quad$ konstanter Korrekturfaktor am Rissrand bei der Risstiefe $a$ (Tab. 6.1)

$\chi_{\mathrm{V}} \quad$ Ausnutzung unter Verkehr; $\chi_{\mathrm{V}}=\sigma_{1, \mathrm{Ed}} / f_{\mathrm{Y}}$

$\sigma_{1, \mathrm{Ed}} \quad$ erste Hauptzugspannung an der Fehlerstelle infolge Verkehrslast als Begleiteinwirkung (Bauteiltemperatur als Leiteinwirkung) 
$\chi_{\mathrm{E}} \quad$ Ausnutzung unter Ermüdung; $\chi_{\mathrm{E}}=\Delta \sigma_{1, \mathrm{E} 2} / f_{\mathrm{y}}$

$\Delta \sigma_{1, \mathrm{E} 2} \quad$ Schwingbreite der ersten Hauptspannung an der Fehlerstelle infolge Ermüdungslast

Folgende Überlegungen liegen der Wahl der Parametergrenzen zugrunde:

- Die Grenzen für die Abmessungen $\gamma$ und $D$ der Knotenstutzen orientieren sich an den Abmessungen der Rundhohlprofile bereits bestehender Fachwerkbrücken mit Stahlgussknoten (Tab. 5.1), wobei für die Knotenstutzen im Untergurt eine kontinuierliche Zunahme der Wanddicke mit einem Winkel von $4^{\circ}$ auf einer Länge von ca. $700 \mathrm{~mm}$ und für die Knotenstutzen der Diagonalen mit einem Winkel von $6.5^{\circ}$ auf einer Länge von $350 \mathrm{~mm}$ mitberücksichtigt wird.

- Für den Brückenbau wird üblicherweise der Stahl S 355 J2H verwendet, in Zukunft dürfte jedoch auch immer häufiger der Feinkornbaustahl S $460 \mathrm{NH}$ zum Einsatz kommen. Baustähle mit Fliessgrenzen $f_{\mathrm{y}}>460 \mathrm{MPa}$ einzusetzen, scheint nicht sinnvoll. Durch den Einsatz von höherfesten Stählen wird zwar für die statische Bemessung Material eingespart. Die Anschlussnähte am Stahlgussknoten unterliegen aber durch die Materialeinsparung höheren Spannungsschwingbreiten und erfordern daher eine höhere Ermüdungsfestigkeit. Diese kann kaum gewährleistet werden, entspricht doch nach derzeitigen Informationen die Ermüdungsfestigkeit der Stumpfschweissnähte höchstens dem Kerbfall 90 (vergleiche Abschnitt 2.4). Die Fliessgrenze des Stahlgusses $f_{\mathrm{y}}$ wird in Anlehnung an diejenige des Stahls der Rundhohlprofile gewählt.

- Die Bruchzähigkeit des gewählten Stahlgusses $K_{\mathrm{Ic}}$ bei einer Betriebstemperatur von $-30^{\circ} \mathrm{C}$ muss die Mindestanforderung der Norm [DIN 17182:1992] erfüllen, also eine Kerbschlagarbeit von mindestens $27 \mathrm{~J}$ bei $-30^{\circ} \mathrm{C}$. Dies entspricht etwa $2400 \mathrm{~N} \cdot \mathrm{mm}^{-3 / 2}$. Die in Abschnitt 3.2.2.2 (auf Seite 31) erwähnten Versuche zur Ermittlung von Bruchzähigkeit und Kerbschlagarbeit zeigen jedoch eine wesentlich höhere Bruchzähigkeit. Aus diesem Grund wird hier eine obere Grenze von $6000 \mathrm{~N} \cdot \mathrm{mm}^{-3 / 2}$ angesetzt.

- Für den Sprödbruchnachweis des mit einem Ermüdungsriss behafteten Stahlgussknotens bei einer Temperatur während der Nutzung von $-30^{\circ} \mathrm{C}$ wird die statische Belastung aus Verkehrslast und Eigengewicht herangezogen. Die Ausnutzung für diesen Lastfall kann wie folgt abgeschätzt werden: Die statische Bemessung erfolgt nach [SIA 260] so, dass bei Belastung mit Verkehr als Leiteinwirkung $\left(\gamma_{Q}=1.50\right)$ und Eigengewicht $\left(\gamma_{G}=1.35\right)$ die Ausnutzung 1.0 ist. Für den Sprödbruchnachweis ist nun die Bauteiltemperatur die Leiteinwirkung, die Verkehrslast wird als Begleiteinwirkung mit $\psi_{1}=0.75$ und das Eigengewicht weiterhin mit $\gamma_{G}=1.35$ angesetzt. Sind die Spannungen aus Eigengewicht und Verkehrslast gleich gross, ergibt sich so eine Ausnutzung für den Sprödbruchnachweis von 0.74. Ist die Spannung aus Verkehrslast grösser als diejenige aus Eigengewicht, sinkt die Ausnutzung noch. Daher wird hier als obere Grenze eine Ausnutzung von 0.75 angenommen. Dieser Wert bezieht sich natürlich auf die Enden der Knotenstutzen; mit zunehmender Wanddicke im Knoteninneren liegt er unter 0.75, da dort die Ausnutzung bereits in der statischer Bemessung nicht 1.0 erreicht. Der Wert von 0.75 entspricht auch der in [prEN 1993-1-10:2003] für den Sprödbruchnachweis bei der tiefsten zulässigen Betriebtemperatur (als Leiteinwirkung) angegebenen maximalen Ausnutzung der Konstruktion.

- Die obere Grenze $\chi_{\mathrm{E}}=0.30$ für die Ausnutzung unter Ermüdungslast entspricht für den Stahl S 355 etwa der Ermüdungsfestigkeit der Schweissnähte mit dem Kerbfall 90 zwischen den Stahlgussknoten und den anschliessenden Rundhohlprofilen $\left(\chi_{\mathrm{E}}=355 \mathrm{MPa} / 90 \mathrm{MPa}=0.25\right)$. 
Tab. 6.3 fasst die für die Parameterstudie verwendeten unteren und oberen Grenzen zusammen.

\begin{tabular}{lcccccc}
\hline & \multicolumn{2}{c}{ Abmessungen } & \multicolumn{2}{c}{ Materialeigenschaften } & \multicolumn{2}{c}{ Belastungszustand } \\
\hline & $\gamma$ & $D$ & $f_{\mathrm{y}}$ & $K_{\mathrm{Ic}}$ & Verkehrslast & Ermüdungslast \\
\cline { 5 - 7 } & {$[-]$} & {$[\mathrm{mm}]$} & {$[\mathrm{MPa}]$} & {$\left[\mathrm{N} \cdot \mathrm{mm}^{-3 / 2}\right]$} & $\chi_{\mathrm{V}}[-]$ & $\chi_{\mathrm{E}}[-]$ \\
\hline untere Grenze & 2 & 200 & 355 & 2000 & 0.45 & 0.10 \\
\hline obere Grenze & 7 & 600 & 460 & 6000 & 0.75 & 0.30 \\
\hline
\end{tabular}

Tab. 6.3: Parametergrenzen

\subsubsection{Algorithmus zur Berechnung der zulässigen Anfangsfehlergrösse}

Das in Abschnitt 5.4 ausführlich beschriebene Vorgehen zur Berechnung der maximal zulässigen Anfangsfehlergrösse wird für die Parameterstudie ebenfalls angewandt. Folgende Anpassungen des Vorgehens wurden für die Parameterstudie gemacht:

- Im Failure Assessment Diagramm geht der maximale Spannungsintensitätsfaktor am Rissrand $K_{\mathrm{I}, \mathrm{ULS}}^{\mathrm{c}}(a)$ für die Berechnung der normierten Grösse $K_{\mathrm{r}}(a)$ mit der nachstehenden Formulierung ein. Der konstante Korrekturfaktor $Y_{\mathrm{c}}$ wird aus Tab. 6.1 entnommen.

$$
K_{\mathrm{I}, \mathrm{ULS}}^{\mathrm{c}}=Y_{\mathrm{c}} \cdot \sigma_{1, \mathrm{Ed}} \cdot \sqrt{\pi \cdot a}
$$

- In der Paris-Erdogan Gleichung geht die Differenz des Spannungsintensitätsfaktors in der Risstiefe $\Delta K_{\mathrm{I}}(a)$ für die Rückrechnung auf die maximal zulässige Anfangsfehlergrösse $a_{0}$ mit der nachstehenden Formulierung ein. Der konstante Korrekturfaktor $Y_{\text {a }}$ wird ebenfalls aus Tab. 6.1 entnommen.

$$
\Delta K_{\mathrm{I}}(a)=Y_{\mathrm{a}} \cdot \Delta \sigma_{1, \mathrm{E} 2} \cdot \sqrt{\pi \cdot a}
$$

Das Vorgehen wird für die Parameterstudie als Algorithmus implementiert. Dies geschieht mit Hilfe der Programmiersprache Visual Basic for Applications (VBA) als benutzerdefinierte Funktion für Microsoft Excel [Microsoft 2003]. Der Algorithmus ist in Anhang C abgedruckt und in Fig. 6.8 schematisch dargestellt. 

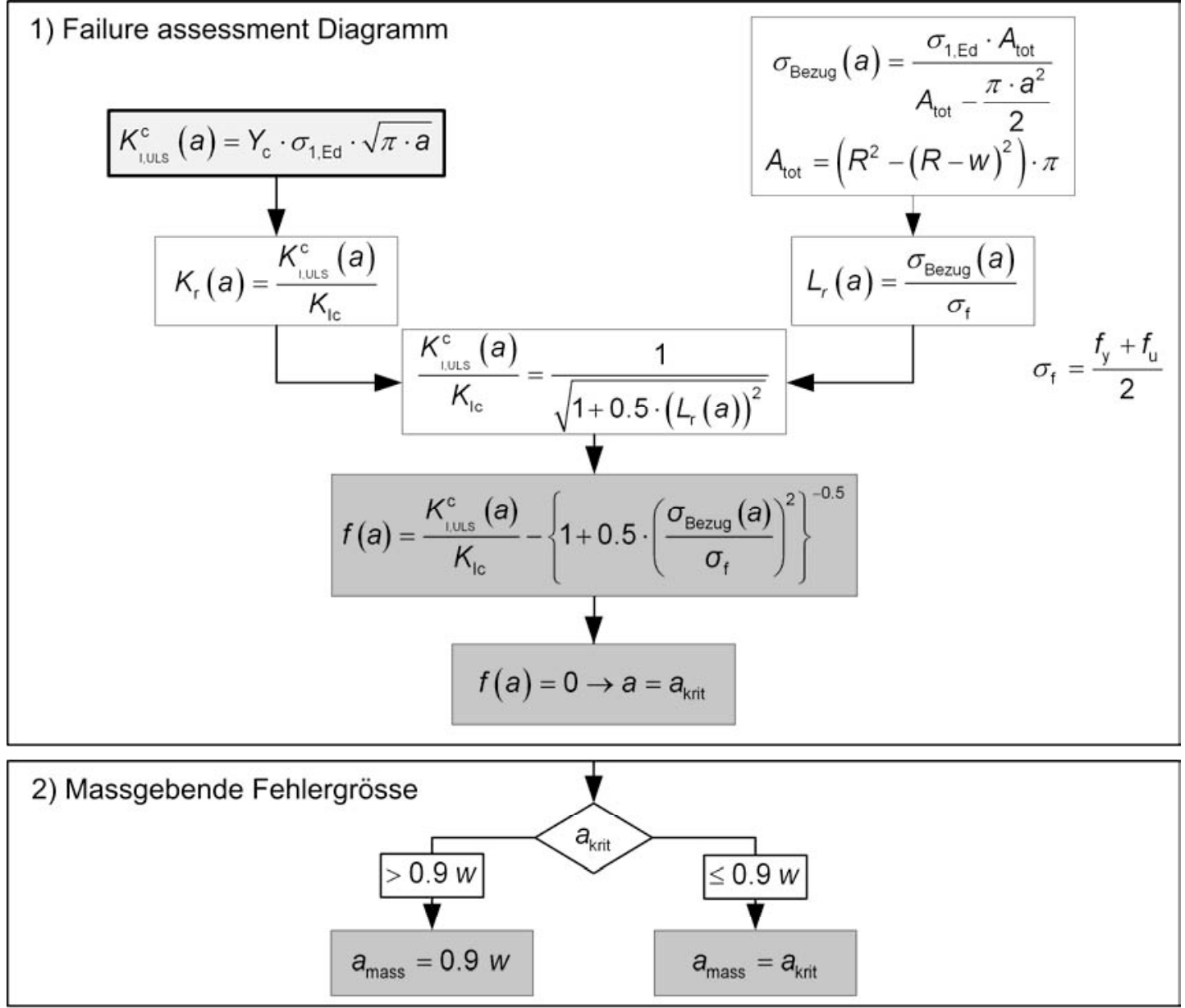

3) Paris-Erdogan Gleichung

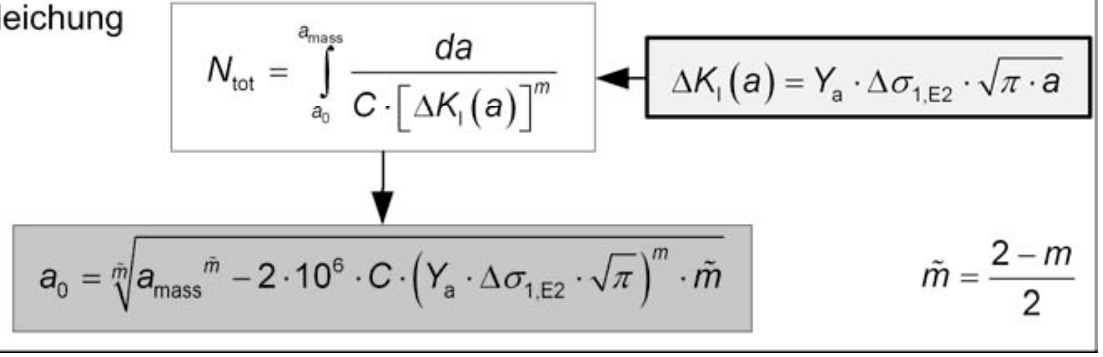

Fig. 6.8: Algorithmus zur Berechnung der maximal zulässigen Anfangsfehlergrösse

\subsubsection{Ergebnisse aus der Parameterstudie}

\subsubsection{Einfluss der Parameter}

Die Ergebnisse der Parameterstudie werden hier nicht tabellarisch wiedergegeben, da sie mit dem Algorithmus für jeden Anwendungsfall berechnet werden können. Interessant ist es hingegen, einen qualitativen Überblick zu erhalten, weshalb nachfolgend der Einfluss der einzelnen Parameter auf die maximal zulässige Anfangsgrösse von Gussfehlern untersucht und erläutert wird.

\section{Einfluss des Belastungszustandes}

Der Belastungszustand ist durch die Ausnutzung unter Verkehr $\chi_{\mathrm{V}}$ (für den Sprödbruchnachweis mit der Bauteiltemperatur als Leiteinwirkung und der Verkehrslast als Begleiteinwirkung) und der Ausnutzung unter Ermüdung $\chi_{\mathrm{E}}$ gegeben. In Fig. 6.9 wird anhand der massgebenden Fehlergrösse 
$a_{\text {mass }}$ und der zulässigen Anfangsfehlergrösse $a_{0}$ der Einfluss der Ausnutzungsgrade $\chi_{\mathrm{V}}$ und $\chi_{\mathrm{E}}$ für ein spezifisches Beispiel $\left(\gamma=2, D=400 \mathrm{~mm}, f_{\mathrm{y}}=355 \mathrm{MPa}, K_{\mathrm{Ic}}=2000 \mathrm{~N} \cdot \mathrm{mm}^{-3 / 2}\right)$ abgebildet.

Die massgebende Fehlergrösse ergibt sich für die gewählten Parameter im gesamten untersuchten Bereich $\left(\chi_{\mathrm{E}}, \chi_{\mathrm{V}}\right)$ durch das Sprödbruchkriterium, es gilt also $a_{\mathrm{mass}}=a_{\text {krit. }}$. Sie ist nur von der Ausnutzung unter Verkehr $\chi_{\mathrm{V}}$ abhängig.

Es ist zudem zu erkennen, dass die maximal zulässige Anfangsfehlergrösse für eine konstante Ausnutzung $\chi_{\mathrm{V}}$ mit zunehmender Ausnutzung $\chi_{\mathrm{E}}$ stark abnimmt. Tatsächlich geht die Spannungsschwingbreite $\Delta \sigma_{1, \mathrm{E} 2}=\chi_{\mathrm{E}} \cdot f_{\mathrm{y}}$ mit dem Exponenten $m=3$ in die Paris-Erdogan Gleichung zur Berechnung der zulässigen Anfangsfehlergrösse $a_{0}$ ein. Dadurch hat die Spannungsschwingbreite einen exponentiell zunehmenden Einfluss bei der Rückrechnung von $a_{\text {mass }}$ auf $a_{0}$.

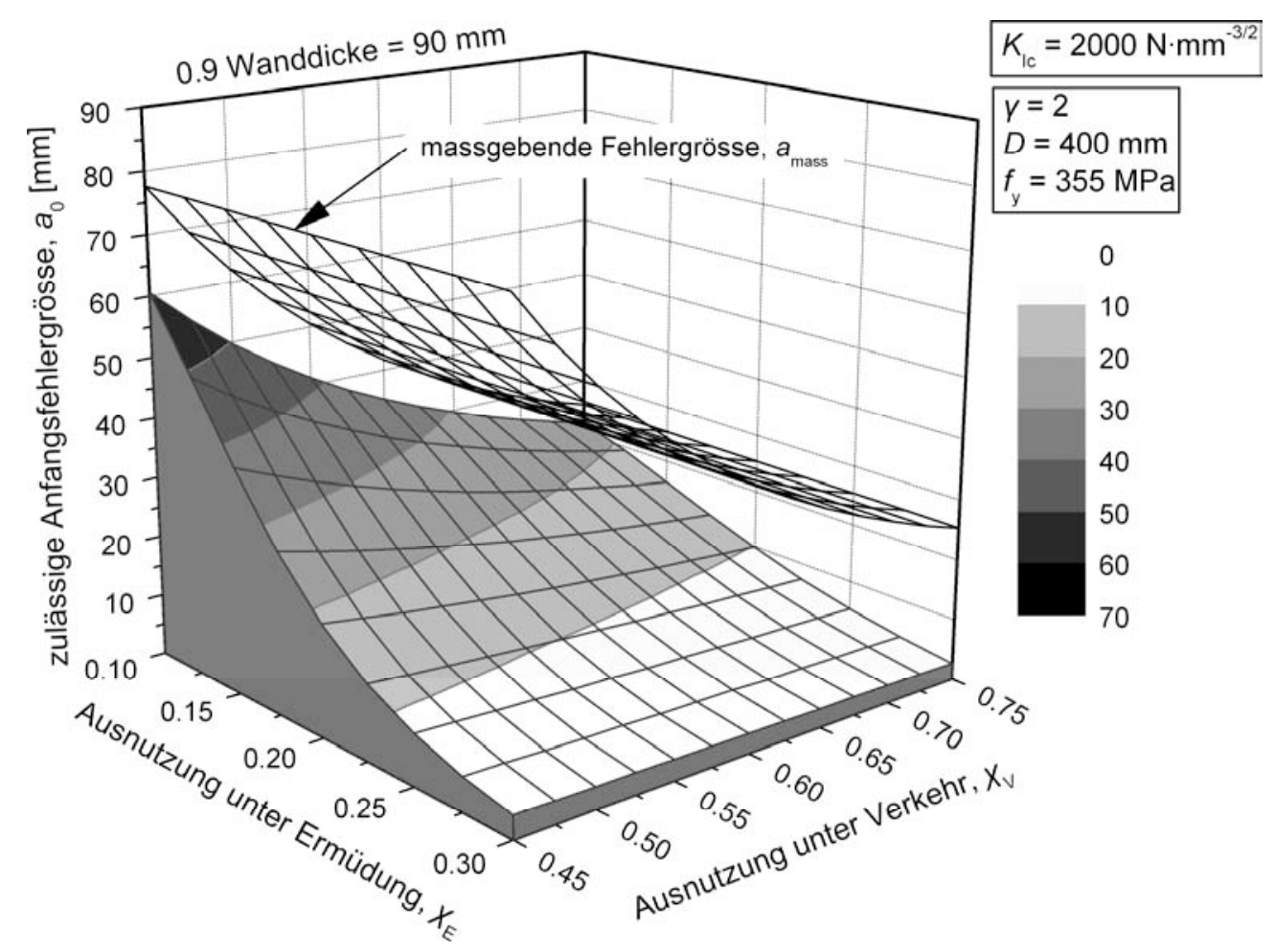

Fig. 6.9: Einfluss der Ausnutzungsgrade $\chi_{\mathrm{E}}$ und $\chi_{\mathrm{V}}$ für $K_{\mathrm{Ic}}=2000 \mathrm{~N} \cdot \mathrm{mm}^{-3 / 2}$

\section{Einfluss der Bruchzähigkeit}

Wird in Fig. 6.10 für das oben erwähnte Beispiel die Bruchzähigkeit auf $K_{\mathrm{Ic}}=2160 \mathrm{Nmm}^{-3 / 2}$ erhöht, so ergibt sich die massgebende Fehlergrösse für $\chi_{\mathrm{V}}=0.45$ durch das Kriterium von $90 \%$ der Wanddicke. Bei $\chi_{\mathrm{V}}=0.45$ werden also mit $K_{\mathrm{Ic}}=2160 \mathrm{Nmm}^{-3 / 2}$ die maximal zulässigen Anfangsfehlergrössen erreicht.

Bei einer weiteren Zunahme von $K_{\text {Ic }}$ steigt die zulässige Anfangsfehlergrösse auch für höhere Ausnutzungen $\chi_{\mathrm{V}}$ auf das Maximum an (Fig. 6.11). Der Einfluss von $a_{\text {mass }}$ (und somit von $K_{\mathrm{Ic}}$ ) auf $a_{0}$ ist bei $\chi_{\mathrm{E}}=0.1$ höher als bei $\chi_{\mathrm{E}}=0.3$. Ab einer Bruchzähigkeit von $K_{\mathrm{Ic}}=3900 \mathrm{Nmm}^{-3 / 2}$ ist für den gesamten untersuchten Bereich $\left(\chi_{\mathrm{E}}, \chi_{\mathrm{V}}\right)$ das Kriterium für die massgebende Fehlergrösse die $90 \%$ der Wanddicke gegeben. Die Ausnutzung unter Verkehr $\chi_{\mathrm{V}}$ hat dann keinen Einfluss mehr. Zusammenfassend kann gesagt werden, dass die Bruchzähigkeit einen grossen Einfluss auf die zulässige 
Anfangsfehlergrösse hat, wenn das Bauteil nur niedriger Ausnutzung unter Ermüdung ausgesetzt ist. Bei hoher Ausnutzung unter Ermüdung ist der Einfluss nur unwesentlich. Der Einfluss der Bruchzähigkeit auf die zulässige Anfangsfehlergrösse für das spezifische Beispiel und für die Ausnutzung unter Ermüdung $\chi_{\mathrm{E}}=0.10$ wird in Fig. 6.12 nochmals veranschaulicht.

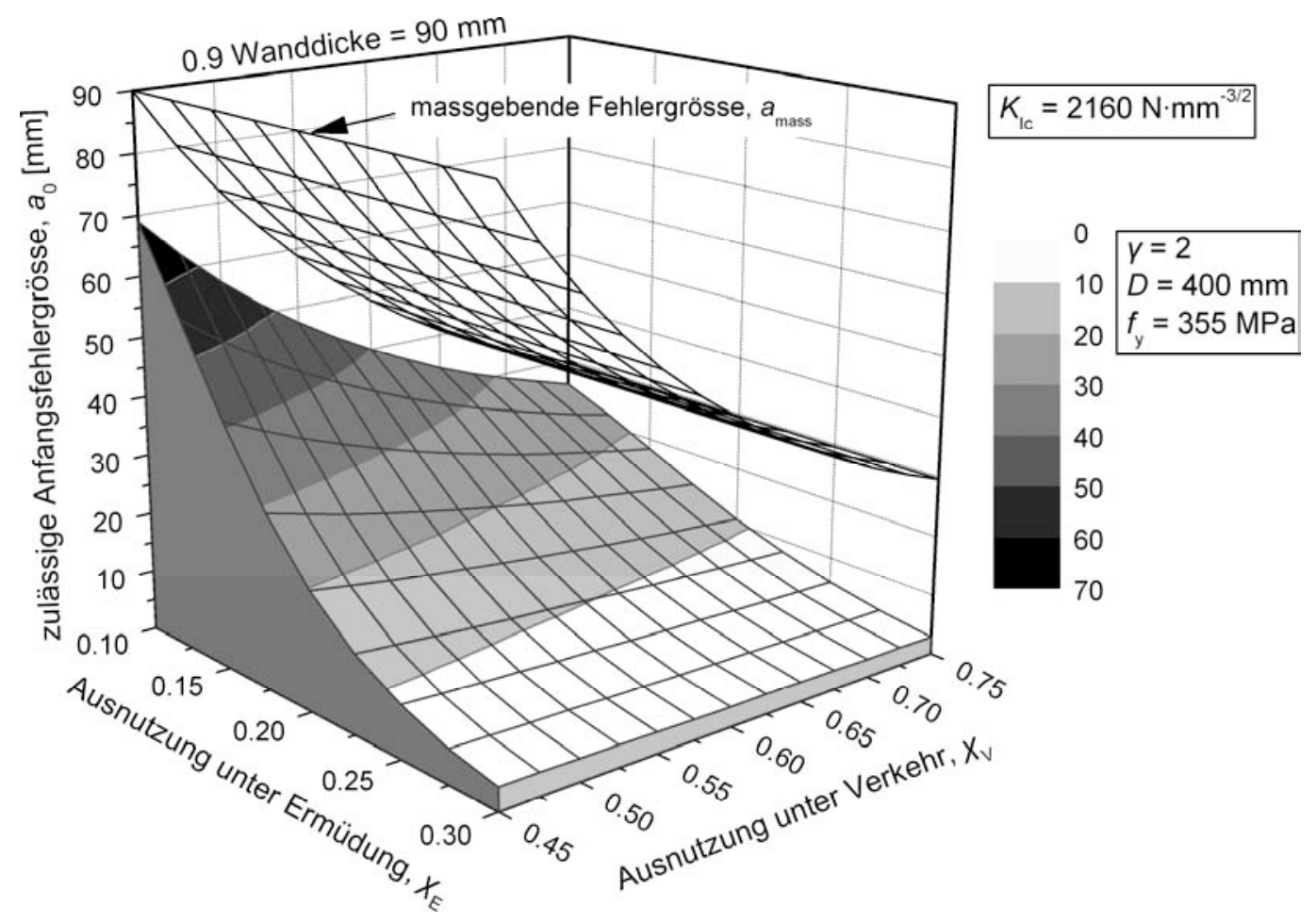

Fig. 6.10: Einfluss der Ausnutzungsgrade $\chi_{\mathrm{E}}$ und $\chi_{\mathrm{V}}$ für $K_{\mathrm{Ic}}=2160 \mathrm{~N} \cdot \mathrm{mm}^{-3 / 2}$

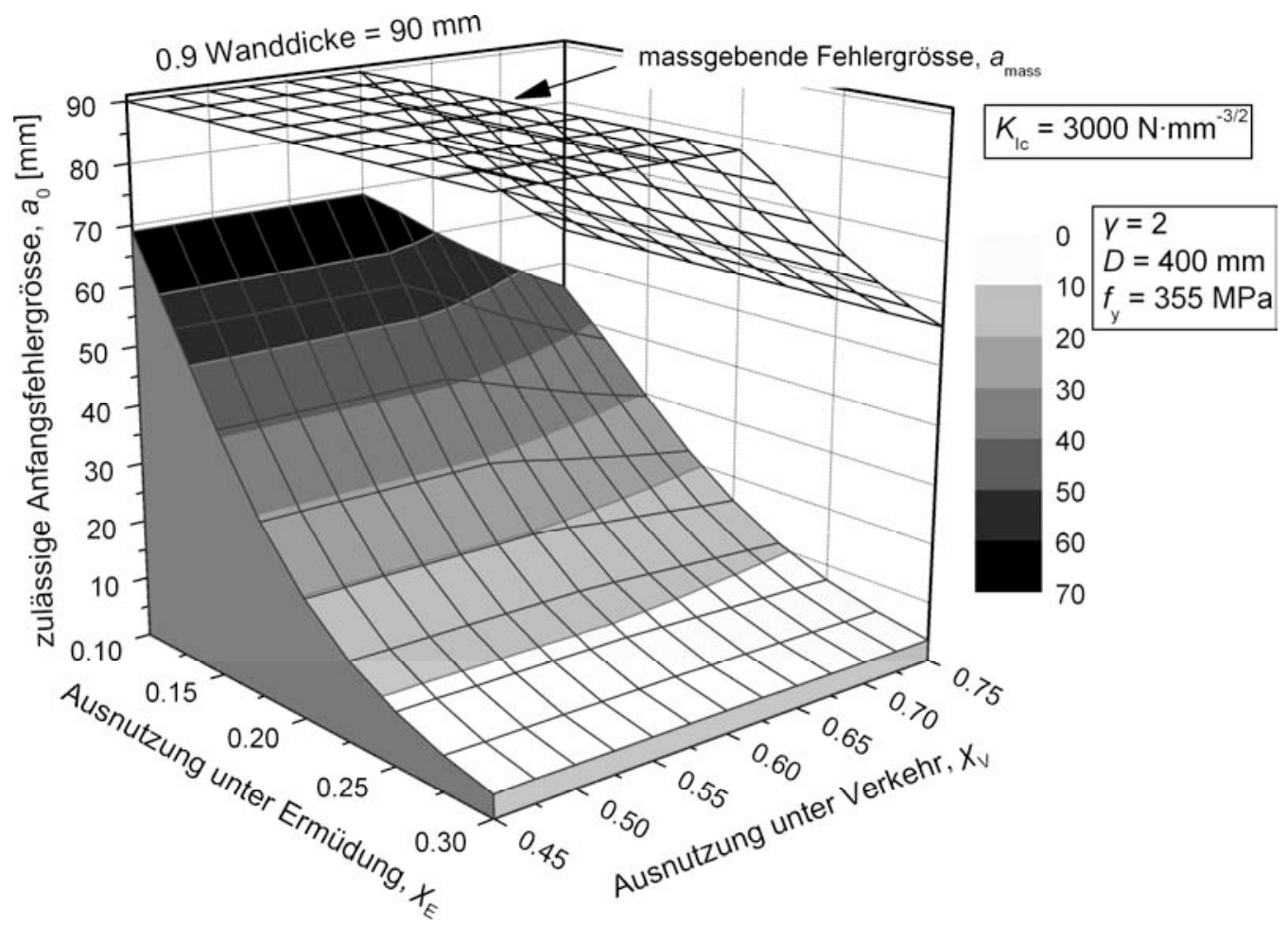

Fig. 6.11: Einfluss der Ausnutzungsgrade $\chi_{\mathrm{E}}$ und $\chi_{\mathrm{V}}$ für $K_{\mathrm{Ic}}=3000 \mathrm{~N} \cdot \mathrm{mm}^{-3 / 2}$ 


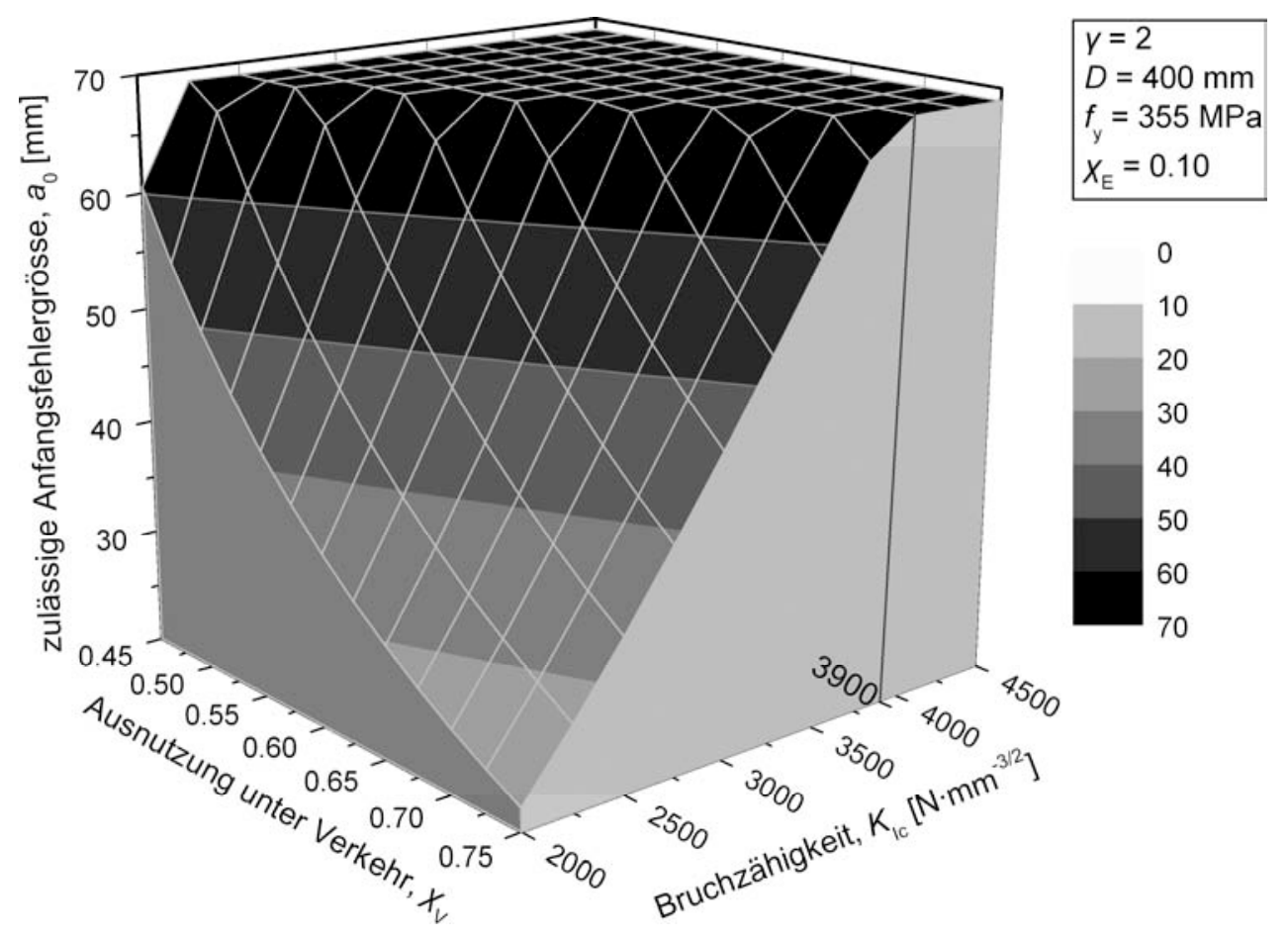

Fig. 6.12: Einfluss der Bruchzähigkeit $K_{\mathrm{Ic}}$ bei $\chi_{\mathrm{E}}=0.1$

\section{Einfluss der Fliessgrenze}

In der Fig. 6.13 wird der Einfluss der Fliessgrenze auf die maximal zulässige Anfangsfehlergrösse $a_{0}$ illustriert. Für die Bruchzähigkeit wird der rechnerische Wert $K_{\mathrm{Ic}}=2400 \mathrm{~N} \cdot \mathrm{mm}^{-3 / 2}$ nach Tab. 3.4 gewählt. Die massgebende Fehlergrösse ergibt sich für dieses Beispiel im gesamten untersuchten Bereich $\left(f_{\mathrm{y}}, \chi_{\mathrm{E}}\right)$ durch das Sprödbruchkriterium mit $a_{\text {mass }}=a_{\text {krit }}$.

Mit zunehmender Fliessgrenze steigt die Spannung $\sigma_{1, \mathrm{Ed}}=\chi_{\mathrm{V}} \cdot f_{\mathrm{y}}$ für den Sprödbruchnachweis und die Spannungsschwingbreite infolge Ermüdungslast $\Delta \sigma_{1, \mathrm{E} 2}=\chi_{\mathrm{E}} \cdot f_{\mathrm{Y}}$ an. Aus diesem Grund nehmen für eine konstante Ausnutzung $\chi_{\mathrm{E}}$ die massgebende Fehlergrösse und die zulässige Anfangsfehlergrösse mit zunehmender Fliessgrenze ab. Die Spannungsschwingbreite $\Delta \sigma_{1, \mathrm{E} 2}=\chi_{\mathrm{E}} \cdot f_{\mathrm{y}}$ geht mit dem Exponenten $m=3$ in die Paris-Erdogan Gleichung zur Berechnung der zulässigen Anfangsfehlergrösse $a_{0}$ ein. Dadurch hat die Spannungsschwingbreite für eine konstante Fliessgrenze einen exponentiell zunehmenden Einfluss bei der Rückrechnung von $a_{\text {mass }}$ auf $a_{0}$.

Es wird angenommen, dass ein Bauteil so bemessen wird, dass es unter Verkehr optimal ausgenutzt wird; $\chi_{\mathrm{V}}$ beträgt daher 0.75 . Wenn für das Bauteil eine höhere Fliessgrenze gewählt wird, steigt die Spannungsschwingbreite infolge der erzielten Materialeinsparung (reduzierte Wanddicke) an. Die Ausnutzung unter Ermüdung bleibt aber unverändert. Durch die reduzierte Wanddicke nimmt automatisch auch die massgebende Fehlergrösse ab. Bei der Zunahme von $f_{\mathrm{y}}$ nimmt daher die Anfangsfehlergrösse infolge der Zunahme von $\Delta \sigma_{1, \mathrm{E} 2}$ und der Abnahme von $a_{\text {mass }}$ sehr stark ab. 


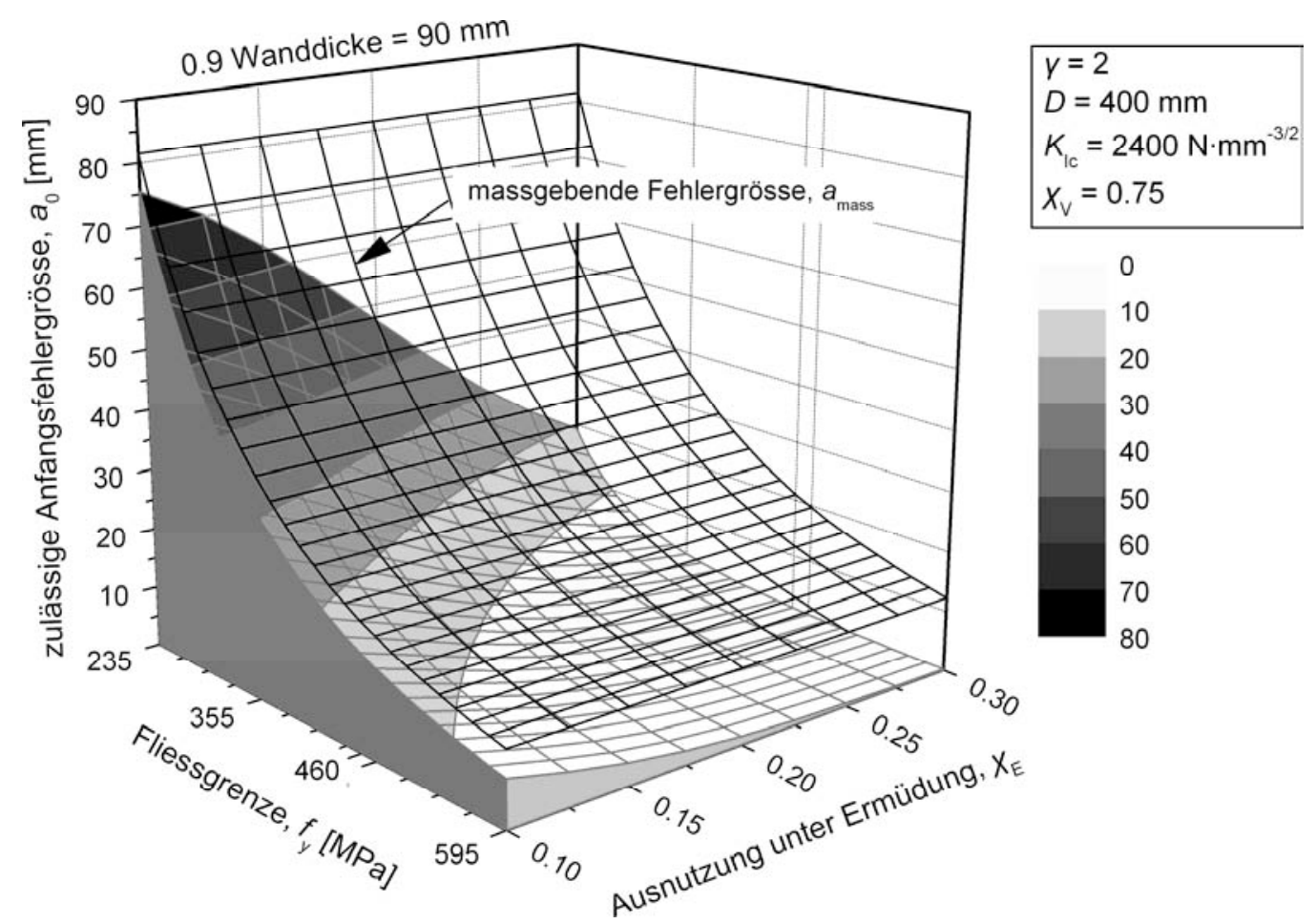

Fig. 6.13: Einfluss der Fliessgrenze $f_{\mathrm{y}}$

\subsubsection{Maximal zulässige Anfangsfehlergrössen für eine mittlere Knotenausnutzung}

Generell wird empfohlen, für konkrete Anwendungen die zulässigen Anfangsgrössen von Gussfehlern für die verschiedenen Bereiche im Knoten direkt mit dem vorgeschlagenen Algorithmus zu berechnen. Dabei soll der Knotenquerschnitt an der Stelle der zu berechnenden Anfangsfehlergrösse durch einen Kreisquerschnitt mit den Parametern $\gamma$ und $D$ angenähert werden.

Um den Bereich von $a_{0}$ abschätzen zu können, werden in diesem Abschnitt für einen plausibel gewählten Wert der Bruchzähigkeit, für eine mittlere Ausnutzung unter Verkehr und unter Ermüdungslast sowie für verschiedene Abmessungen $\gamma$ und $D$ numerische Werte der maximal zulässigen Anfangsfehlergrösse $a_{0}$ zusammengefasst.

Für die Bruchzähigkeit wird hier nicht der rechnerische (siehe Tab. 3.4), sondern ein wahrscheinlicher Wert gewählt. Die Auswertung der Ergebnisse der Kerbschlagarbeit $A_{v}$ für die Prüftemperatur von $-30^{\circ} \mathrm{C}$ aus internen Prüfprotokollen der Stahlgiesserei Friedrich Wilhelms-Hütte [FWH 2004] ergibt einen Mittelwert von 103 J mit einer Standardabweichung von 17 J. Die Ergebnisse folgen einer Normalverteilung. Daraus ergibt sich nach Gleichung (3.3) (auf Seite 33) eine Bruchzähigkeit von $K_{\mathrm{Ic}}=4700 \mathrm{~N} \cdot \mathrm{mm}^{-3 / 2}$. Die Bruchzähigkeit wird unabhängig von der Fliessgrenze gewählt.

Auf Grund der giesstechnisch bedingt dickeren Wanddicken (siehe Abschnitt 3.2.2) ist die Ausnutzung der Stahlgussknoten tiefer als diejenige der anschliessenden Hohlprofile. Daher wird hier für die Stahlgussknoten nur ein mittlerer Belastungszustand mit einer Ausnutzung unter Ermüdungslast $\chi_{\mathrm{E}}=0.20$ und unter Verkehr $\chi_{\mathrm{V}}=0.60$ angenommen. In Fig. 6.14 und Fig. 6.15 werden in Abhängigkeit der Knotengeometrie $(\gamma, D)$ und für die Fliessgrenzen $f_{\mathrm{y}}=355 \mathrm{MPa}$ und $f_{\mathrm{y}}=460 \mathrm{MPa}$ die zulässigen Anfangsfehlergrössen $a_{0}$ für diesen mittleren Belastungszustand angegeben. 


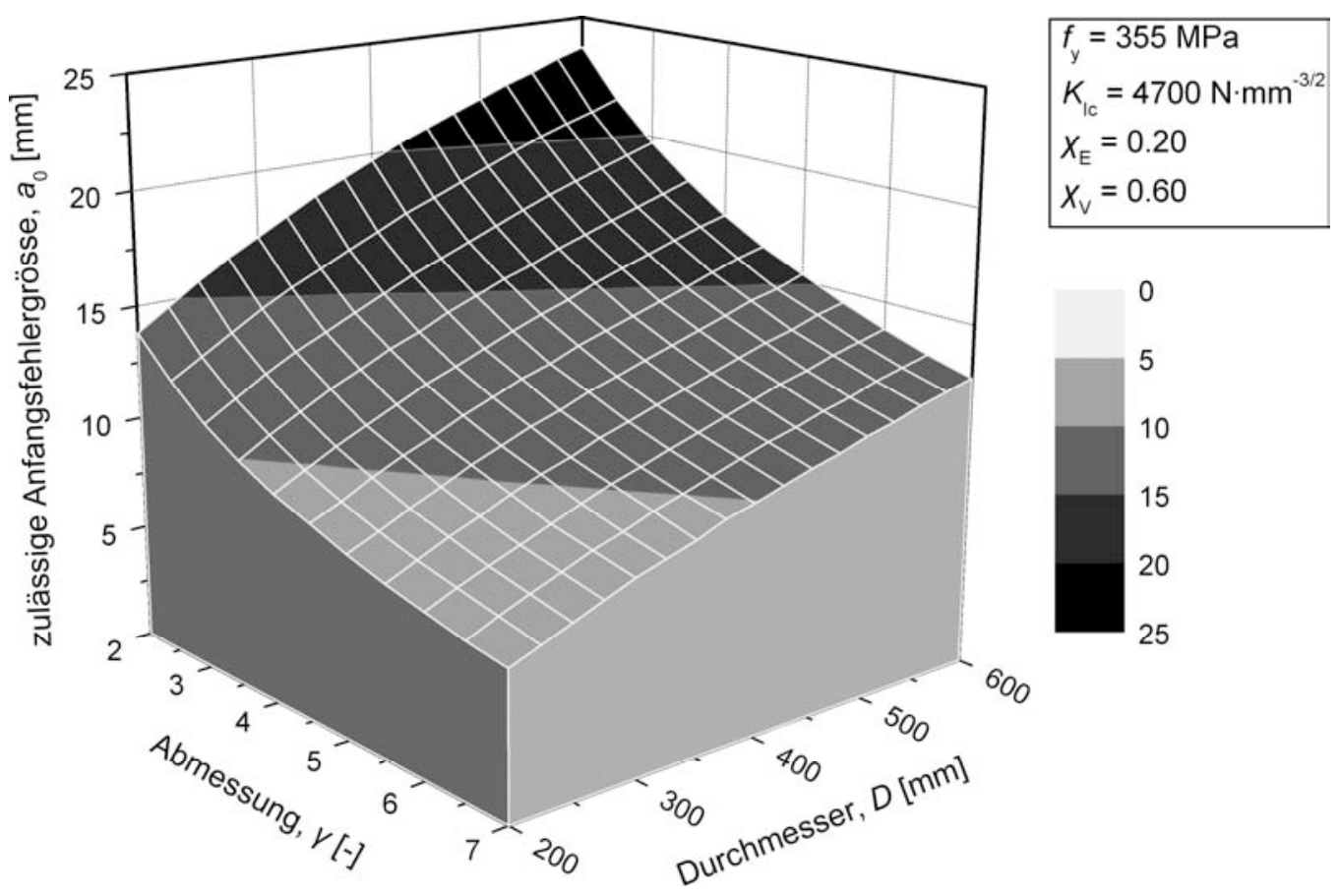

Fig. 6.14: Zulässige Anfangsfehlergrössen in Abhängigkeit der Geometrie für die Fliessgrenze $f_{y}=355 \mathrm{MPa}$

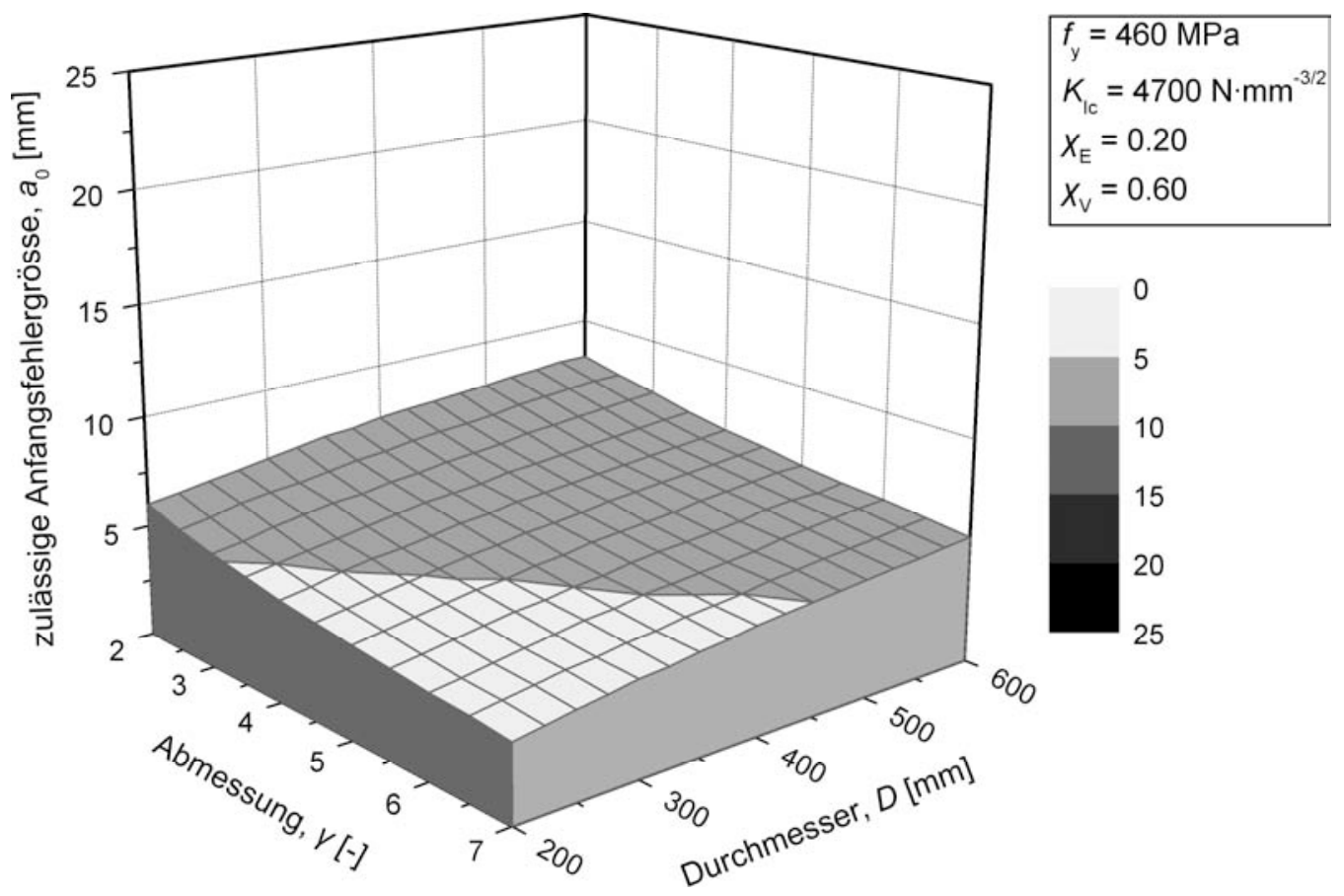

Fig. 6.15: Zulässige Anfangsfehlergrössen in Abhängigkeit der Geometrie für die Fliessgrenze $f_{y}=460 \mathrm{MPa}$

Für beide Fliessgrenzen ist die massgebende Fehlergrösse $a_{\text {mass }}$ im gesamten untersuchten Bereich $(\gamma, D)$ durch die $90 \%$ der Wanddicke begrenzt (in Fig. 6.14 und Fig. 6.15 nicht dargestellt). Demnach hat die Ausnutzung unter Verkehr $\chi_{\mathrm{v}}$ keinen Einfluss auf $a_{\text {mass. }}$. 
Es ist zu beobachten, dass die Anfangsfehlergrössen $a_{0}$ für die Fliessgrenze $f_{\mathrm{y}}=355 \mathrm{MPa}$ im gesamten Bereich $(\gamma, D)$ zwischen $6.3 \mathrm{~mm}$ und $23.6 \mathrm{~mm}$ liegen. Für die Fliessgrenze $f_{\mathrm{y}}=460 \mathrm{MPa}$ liegen die Anfangsfehlergrössen im Bereich $3.4 \mathrm{~mm} \leq a_{0} \leq 8.3 \mathrm{~mm}$. Bei der Anwendung dieser Werte für konkrete Anwendungen muss beachtet werden, dass für die vorstehenden Betrachtungen die Ausnutzung über den gesamten Abmessungsbereich als konstant angenommen wurde. Da in einem Stahlgussknoten aus giesstechnischen Gründen die Wanddicken zur Knotenmitte hin zunehmen (vergleiche Abschnitt 3.2.2), nimmt die Ausnutzung mit zunehmender Wanddicke ab. Der Knotenquerschnitt mit der unteren Grenze $\gamma=2$, der oberen Grenze $D=600 \mathrm{~mm}$ und damit der maximalen Wanddicke $w=150 \mathrm{~mm}$ befindet sich bei üblichen K- oder KK-Knoten im Bereich der Knotenmitte. Für diesen Querschnitt ist daher mit einer weit niedrigeren Ausnutzung als die vorgeschlagenen Werte $\chi_{\mathrm{E}}=0.20$ und $\chi_{\mathrm{V}}=0.60 \mathrm{zu}$ rechnen. Daraus folgt, dass die zulässigen Anfangsfehlergrössen weit über den angegebenen $23.6 \mathrm{~mm}\left(f_{\mathrm{y}}=355 \mathrm{MPa}\right)$ bzw. $8.3 \mathrm{~mm}\left(f_{\mathrm{y}}=460 \mathrm{MPa}\right)$ liegen. Der Knotenquerschnitt mit $\gamma=7, D=200 \mathrm{~mm}$ und damit der minimalen Wanddicke $w=14 \mathrm{~mm}$ liegt hingegen im Bereich der Anschweissenden. Im Verhältnis zu dieser Wanddicke sind die zulässigen Anfangsfehlergrössen $a_{0}=6.3 \mathrm{~mm}\left(f_{\mathrm{y}}=355 \mathrm{MPa}\right)$ und $a_{0}=3.4 \mathrm{~mm}\left(f_{\mathrm{y}}=460 \mathrm{MPa}\right)$ sehr gross.

\subsubsection{Betrachtungen zum Zusammenhang zwischen zulässiger Anfangsrissgrösse und Gütestufe}

Es ist nun interessant, den Bezug der zulässigen Anfangsfehlergrössen zu den Gütestufen der Stahlgussknoten nach [DIN 1690-2:1985] herzustellen. In Tab. 2.2 auf Seite 16 werden für die fünf Gütestufen verschiedene höchstzulässige Anzeigenmerkmale bei der Ultraschallprüfung festgelegt. Ein Anzeigenmerkmal ist das maximal zulässige Verhältnis der Ausdehnung der Reflektionsstelle zur Wanddicke des Gussstücks an der geprüften Stelle. Dabei sind für die Gütestufen 2 bis 4 Gussfehler (= Reflektionsstellen) mit einer Ausdehnung von maximal $10 \%$ der Wandstärke $w$ für die beiden Randzonen und von maximal $15 \%$ für die Kernzone zugelassen. Für die am wenigsten strenge Gütestufe 5 werden für Rand- und Kernzone sogar 15 bzw. 25 \% der Wanddicke zugelassen. Diese sollen nun mit den berechneten Anfangsfehlergrössen verglichen werden. Für den Vergleich wird angenommen, dass die Anfangsfehlergrössen mit Ultraschall detektiert werden sollen, damit deren Ausdehnung in Dickenrichtung gemessen werden kann.

\begin{tabular}{lccccc}
\hline & \multicolumn{2}{c}{$f_{\mathrm{y}}=355 \mathrm{MPa}$} & \multicolumn{2}{c}{$f_{\mathrm{y}}=460 \mathrm{MPa}$} \\
\hline geometrischer Parameter: $\gamma=D / 2 w$ & {$[-]$} & 7 & 2 & 7 & 2 \\
Durchmesser: $D$ & {$[\mathrm{~mm}]$} & 200 & 600 & 200 & 600 \\
Wanddicke: $w$ & {$[\mathrm{~mm}]$} & 14 & 150 & 14 & 150 \\
\hline$a_{0}$ gemäss Fig. 6.14 und Fig. 6.15 & {$[\mathrm{~mm}]$} & $\mathbf{6 . 3}$ & $\mathbf{2 3 . 6}$ & $\mathbf{3 . 4}$ & $\mathbf{8 . 3}$ \\
\hline Dicke der Kern- $\left(w_{\mathrm{K}}\right)$ bzw. Randzone $\left(w_{\mathrm{R}}\right)$ & {$[\mathrm{mm}]$} & 4.7 & 50 & 4.7 & 50 \\
Anzeigenmerkmal Kernzone: $a_{0, \mathrm{~K}}=0.25 w_{\mathrm{K}}$ & {$[\mathrm{mm}]$} & 1.2 & 12.5 & 1.2 & 12.5 \\
Anzeigenmerkmal Randzone: $a_{0, \mathrm{R}}=0.15 w_{\mathrm{R}}$ & {$[\mathrm{mm}]$} & $\mathbf{0 . 7}$ & $\mathbf{7 . 5}$ & $\mathbf{0 . 7}$ & $\mathbf{7 . 5}$ \\
\hline
\end{tabular}

Tab. 6.4: Vergleich der Anfangsfehlergrössen mit der Gütestufe 5 nach [DIN 1690-2:1985]

In Tab. 6.4 werden die Anfangsfehlergrössen für verschiedene Fliessgrenzen und Querschnitte zusammengefasst. Als Vergleich sind die zulässigen Anzeigenmerkmale der Gütestufe 5 für die Kernund Randzone angegeben. Da die zulässigen Anfangsfehlergrössen Oberflächenfehler (Abschnitt 
6.3.1) sind, werden sie mit dem zulässigen Anzeigenmerkmal für die Randzone $\left(a_{0, \mathrm{R}}=0.15 w_{\mathrm{R}}\right)$ verglichen.

Es ist zu erkennen, dass die berechneten zulässigen Anfangsfehlergrössen alle wesentlich über dem zulässigen Anzeigenmerkmal liegen. Demnach ist für das betrachtete Anzeigenmerkmal sogar die Gütestufe 5 zu konservativ. Zusätzlich könnten die zulässigen Anfangsfehlergrössen durch einen kleineren Oberflächenfehler und einen Innenfehler, welche bei gegenseitiger Interaktion der Grösse des ursprünglichen Anfangsfehlers entsprechen, ersetzt werden. In [BS 7910:1999] werden dazu Abstands- und Grössenmasse angegeben. Diese Fehler müssten ebenfalls mit dem zulässigen Anzeigenmerkmal verglichen werden. Es genügt jedoch nicht, nur den Zusammenhang zwischen der grössten Ausdehnung der Reflexionsstelle in Wanddickenrichtung und der berechneten zulässigen Anfangsfehlergrösse herzustellen. Es müssten auch die anderen Anzeigenmerkmale aus Tab. 2.2 wie die grösste Länge, die grösste Einzel- oder Gesamtfläche berücksichtigt werden. Diese Betrachtungen zeigen auf, dass der Zusammenhang zwischen den berechneten zulässigen Anfangsfehlergrössen und den Gütestufen nach [DIN 1690-2:1985] [SN EN 12680-1:2003] [SN EN 12680-2:2003] nicht direkt hergestellt werden kann. Dies rührt wahrscheinlich auch daher, dass die Gütestufen nicht bezüglich der Ermüdungsbeanspruchung ausgelegt worden sind. Es müssten also Qualitätsstufen eingeführt werden, die direkt hinsichtlich der Ermüdung (Gussfehlerart, -grösse, -lage, Abstand zwischen zwei Gussfehlern) angepasst sind. Es stellt sich nun die Frage, wie die Gütestufen der Stahlgussknoten für die Bestellung bei der Stahlgiesserei ausgewählt werden sollen.

Eine Möglichkeit könnte darin bestehen, dass tabellarisch festgelegt wird, welche rechnerischen Anfangsfehlergrössen pro Gütestufe und Wanddickenbereich maximal zulässig sind. Durch den Vergleich der in Abhängigkeit der Fliessgrenze, der Ausnutzungen unter Verkehrs- und Ermüdungslast, der Bruchzähigkeit und der Querschnittsparameter berechneten Anfangsfehlergrösse (mit Hilfe des Algorithmus in Anhang C) mit den in der Tabelle aufgeführten Werten kann die erforderliche Gütestufe ausgewählt werden. In diesem Fall würde die Wahl der Gütestufe nur von der zulässigen Anfangsfehlergrösse abhängen.

Eine weitere Möglichkeit für die Auswahl der Gütestufe könnte ähnlich dem in [prEN 1993-110:2003] dargestellten Prinzip zur Stahlsortenauswahl im Hinblick auf die Sprödbruchsicherheit sein. Dieses Prinzip besteht darin, in Abhängigkeit der Betriebstemperatur, der Ausnutzung unter statischer Last, der Stahlsorte und der Gütegruppe die grösste zulässige Erzeugnisdicke eines geschweissten Konstruktionsdetails anzugeben. Die Bewertungsgruppe (B, C und D) der Schweissnaht wird dabei nicht mitberücksichtigt. In einer Tabelle werden die grössten zulässigen Erzeugnisdicken zusammengefasst. Der Sprödbruchnachweis reduziert sich damit auf die angemessene Auswahl der zulässigen Erzeugnisdicke. In diesem Sinne könnte auch die Auswahl der Gütestufe tabellarisch in Abhängigkeit der Fliessgrenze, der Ausnutzungen unter Verkehrslast und Ermüdungslast, der Bruchzähigkeit und der Querschnittsparameter zusammengefasst werden. Die tabellarische Zusammenstellung bietet den Vorteil, dass für die Ermüdungsbemessung keine Berechnung der zulässigen Anfangsfehlergrösse notwendig ist.

Im Rahmen dieser Arbeit kann diese Thematik nicht abschliessend behandelt werden. Es besteht diesbezüglich noch wesentlicher Forschungsbedarf. 


\subsubsection{Betrachtungen zur Detektierbarkeit der maximal zulässigen Anfangsfehlergrössen}

Ein weiterer wichtiger Aspekt bei der Festlegung der zulässigen Anfangsfehlergrössen ist deren Detektierbarkeit mit zerstörungsfreien Prüfungen. Es ist sinnlos, Anfangsfehlergrössen festzulegen, die mit zerstörungsfreien Prüfungen nicht detektiert werden können. Solche Grössen dürften im Rahmen eines Ermüdungsnachweises nicht verwendet werden. In Tab. 2.1 auf Seite 15 werden die Nachweisgrenzen von Fehlern für die verschiedenen Prüfmethoden zusammengefasst.

Für die Durchstrahlungs-, die Magnetpulver- und die Farbeindringprüfung sind „exakte“ Werte verfügbar. Für die Ultraschallprüfung entspricht hingegen die Nachweisgrenze der Risstiefe dreimal der Oberflächenrauheit des untersuchten Gussstücks unter der Bedingung, dass das Echo der Fehlerstelle mindestens zweimal so gross wie der Störpegel ist. Je grösser also die Oberflächenrauheit, desto grösser ist auch die Nachweisgrenze der Fehler.

In den entsprechenden Normen [DIN 1690-2:1985] [SN EN 12680-1:2003] [SN EN 12680-2:2003] wird daher vorgeschrieben, dass die Gussstückoberflächen eine einwandfreie Ankopplung des Prüfkopfs ermöglichen müssen. Dafür muss die Oberflächenbeschaffenheit bestimmten Vergleichsmustern nach [DIN EN 1370:1996] entsprechen. Mit Hilfe der Vergleichsmuster können keine Oberflächenkennwerte wie Rauhtiefe, Mittenrauheit oder Profiltiefe für die Beurteilung der Rauhheit von Gussoberflächen ermittelt werden. Die Rauhtiefe dieser Vergleichsmuster liegt im Bereich von Mikrometern, konkrete Zahlenwerte werden aber nicht gegeben. In [Christianus et al. 1998] wird darauf hingewiesen, dass bei der Erarbeitung der [DIN EN 1370:1996] auf die ursprüngliche Absicht, den verschiedenen Formverfahren, Giessverfahren und Gusswerkstoffen konkrete Zahlenwerte für die Oberflächenrauhheit zuzuordnen, zum gegenwärtigen Zeitpunkt verzichtet worden sei. Für eine derartige Präzisierung - wenn sie überhaupt möglich ist - seien noch umfangreiche Untersuchungen notwendig.

Die Ultraschallprüfbarkeit von Gussstücken hängt nicht nur von der Oberflächenbeschaffenheit sondern auch vom Gefügezustand im Inneren und von der Wanddicke ab. In den Normen [DIN 1690-2:1985] [SN EN 12680-1:2003] [SN EN 12680-2:2003] wird daher vorgeschrieben, dass die Ultraschallprüfbarkeit des Stahlgusses durch Vergleich der Echohöhe eines Bezugsreflektors mit dem durch das Materialgefüge bedingten Störpegel beurteilt werden muss. Damit die Ultraschallprüfbarkeit gewährleistet ist, muss die Echohöhe des Bezugsreflektors mindestens $6 \mathrm{~dB}$ über dem Störpegel liegen. Der Bezugsreflektor - auch Ersatzreflektor genannt - ist ein idealer Fehler (Flachbodenbohrung) mit einem bestimmten Durchmesser. Bei der Prüfung eines Gussstücks wird das Echo einer Reflektorstelle mit demjenigen des Ersatzreflektors verglichen. Wenn das Echo der Reflektorstelle grösser ist als dasjenige des Ersatzreflektors, wird die Reflektorstelle als Fehler registriert. Aus dem Echo einer Fehlerstelle kann jedoch nicht direkt auf die Fehlerart und auf die Ausdehnung in Wanddickenrichtung geschlossen werden. Nur im Idealfall entspricht die Grösse und Form der Fehlerstelle dem Durchmesser des Ersatzreflektors. Inwiefern in der Praxis Fehler detektiert werden können, deren Ausdehnungen kleiner sind als der Durchmesser des Ersatzreflektors, ist nicht klar.

Je nach Wanddicke des Gussstücks wird der kleinste nachweisbare Bezugsreflektordurchmesser vorgeschrieben. Bei hochbeanspruchten Stahlgussstücken mit einer Wanddicke $\leq 100 \mathrm{~mm}$ [SN EN 12680-2:2003] muss zum Beispiel ein Bezugsreflektor mit einem Durchmesser von $2 \mathrm{~mm}$ noch nachgewiesen werden können. Für Anschweissenden wird ein Bezugsreflektor mit einem Durch- 
messer von $1.5 \mathrm{~mm}$ vorgeschrieben. In einer zusätzlichen Prüfanweisung können auch kleinere Bezugsreflektordurchmesser zwischen dem Kunden und der Stahlgiesserei vereinbart werden.

Die im Abschnitt 6.3.3.2 berechneten zulässigen Anfangsfehlergrössen sind grösser als die genannten Durchmesser der Bezugsreflektoren. Aus den oben genannten Gründen ist abzuklären, ob diese Fehlergrössen tatsächlich detektiert werden können.

\subsection{VORGEHEN FÜR DIE ERMÜDUNGSBEMESSUNG}

Dank der Näherungsformel vereinfacht sich das in Kapitel 5 anhand der typischen Verbundbrücke dargestellte Vorgehen zur Ermüdungsbemessung von Stahlgussknoten wesentlich. Im Folgenden werden die wichtigsten Schritte gegliedert und kurz beschrieben.

1. Ein Stabmodell der Fachwerkbrücke wird erstellt, wobei die Stahlgussknoten ebenfalls durch Stäbe modelliert werden. Aus der anfänglich gewählten Knotenform werden für die Knotenstutzen unter Beibehaltung der variablen Wanddicken die entsprechenden Ersatzstäbe herausgelesen. Die Ersatzstäbe des Knotens sind im Stabmodell der Brücke eingespannt. Es wird empfohlen, bei der Wahl der Knotenform die Knotenstutzen mit minimaler Länge auszuführen, um Herstellungskosten zu sparen. Anhand des Stabmodells werden die Schnittkräfte unter den ständigen und veränderlichen Einwirkungen berechnet.

2. Die Brücke wird hinsichtlich der Tragsicherheit und der Gebrauchstauglichkeit nach den aktuellen Normen bemessen.

3. Für die Ermüdungsbemessung werden zunächst nur die Stumpfschweissnähte zwischen den Hohlprofilen und den Stutzen der Stahlgussknoten betrachtet. Der Ermüdungsnachweis der Schweissnähte erfolgt mit Hilfe von Ermüdungsfestigkeitskurven (Wöhlerkurven). Aus der statischen Bemessung und der Ermüdungsbemessung ergeben sich die nötigen Durchmesser und Wanddicken der Hohlprofile, mit denen anschliessend die Knotenform in Absprache mit der Stahlgiesserei definiert wird.

4. Die Bestimmung der maximal zulässigen Anfangsgrösse von Gussfehlern für eine Lebensdauer von 70 Jahren (entspricht $2 \cdot 10^{6}$ Lastwechseln, analog den Stumpfschweissnähten) und beinhaltet folgende Schritte:

- Die für die Ermüdungsbemessung massgebenden Stahlgussknoten werden nach der grössten Zugkraft im Untergurtstutzen und im Diagonalstutzen infolge der Überfahrt der Ermüdungslast über die Brücke gewählt.

- Ein numerisches Modell des Knotens kann mit finiten Elementen oder Randelementen erstellt werden. Das Modell dient nur zur Berechnung der Spannungen im Knoten, auf Rissfortschrittsberechnungen kann dank der Näherungsformel verzichtet werden.

- Die zu untersuchenden Knoten werden in verschiedene Bereiche aufgeteilt, für die die zulässige Anfangsfehlergrösse von Gussfehlern definiert werden soll. In jedem Bereich wird der Stelle, die querschnitts- oder geometriebedingt die höchsten Spannungen aufweist, ein Gussfehler zugewiesen, für den die Anfangsgrösse berechnet werden soll. Der Gussfehler wird entweder im Inneren oder an der Oberfläche als kreis- bzw. halbkreisförmiger Riss angesetzt und ist senkrecht zur ersten Hauptspannungsrichtung ausgerichtet.

- Für den Sprödbruchnachweis ist die Bauteiltemperatur die Leiteinwirkung, die Verkehrslast wird als Begleiteinwirkung mit $\psi_{1}=0.75$ und das Eigengewicht mit $\gamma_{G}=1.35$ angesetzt. Die erste 
Hauptspannung $\sigma_{1, \mathrm{Ed}}$ für diese Lastkombination wird für jede Fehlerstelle berechnet. Dazu werden die Schnittkräfte aus dem Stabmodell der Brücke übernommen und im numerischen Knotenmodell angesetzt.

- Die Einflusslinie der ersten Hauptzugspannung an den Fehlerstellen infolge der Überfahrt der Ermüdungslast über die Brücke wird berechnet. Dazu werden die Axialkräfte und die dazugehörenden Biegemomente (unter Vernachlässigung der Querkräfte) infolge der Ermüdungslast, die aus dem Stabmodell der Brücke entnommen werden kann, im numerischen Knotenmodell angesetzt. Für einen Innenriss werden die Spannungen im Rissmittelpunkt, für einen Oberflächenriss im auf die Oberfläche projizierten Rissmittelpunkt ermittelt. Anhand der Einflusslinien wird das Maximum und das Minimum der ersten Hauptzugspannung an der Fehlerstelle (unter Berücksichtigung ev. Spannungskonzentration) ermittelt, woraus sich die für die Risswachstumsberechnung erforderliche Schwingbreite der ersten Hauptspannung $\Delta \sigma_{1, \mathrm{E} 2}$ ergibt.

- Mit den Angaben zur Schwingbreite der ersten Hauptspannung infolge der Ermüdungslast, der ersten Hauptspannung aus der Bemessungssituation für den Sprödbruch, den Angaben zum Querschnitt, in dem sich der Fehler befindet, der rechnerischen Fliessgrenze, der rechnerischen Bruchzähigkeit und den konstanten Korrekturfaktoren kann nun mit Hilfe des in Abschnitt 6.3.2 beschriebenen Algorithmus die zulässige Anfangsfehlergrösse für alle Fehlerstellen berechnet werden.

\subsection{FOLGERUNGEN}

Die Einführung einer Näherungsformel für den Spannungsintensitätsfaktor, die auf der Annahme eines konstanten Korrekturfaktors $Y$ beruht, ermöglicht eine gute und einfache Abschätzung der zulässigen Anfangsfehlergrössen von Stahlgussknoten, ohne dass zeitaufwendige numerische Rissfortschrittssimulationen durchgeführt werden müssen. Aus den Untersuchungen können folgende Schlüsse gezogen werden:

- Für Oberflächenrisse muss nicht nach Lage der Risse auf der Aussen- oder Innenseite der Gussknoten unterschieden werden, da sich die Spannungsintensitätsfaktoren nicht wesentlich unterschieden.

- Für den Oberflächenriss unterscheiden sich die Korrekturfaktoren in der Risstiefe $Y_{\mathrm{a}}=0.67$ und am Rissrand $Y_{c}=0.77$. Dies bedeutet, dass der Riss in die Länge etwas schneller wächst als in die Tiefe. Für den Innenriss sind die Korrekturfaktoren $Y_{\mathrm{a}}=0.62$ und $Y_{\mathrm{c}}=0.64$ fast identisch, was für einen kreisrunden Innenriss charakteristisch ist.

- Die Auswertung der Berechnungen zeigt, dass eine vereinfachte, näherungsweise Berechnung von $K_{\mathrm{I}}$ mit einem konstanten Korrekturfaktor möglich ist. Der Vergleich zwischen den mit dem Programm BEASY und mit der Näherungsformel berechneten zulässigen Anfangsfehlergrössen zeigt im Allgemeinen Abweichungen von deutlich unter $\pm 20 \%$.

Durch die Näherungsformel für den Spannungsintensitätsfaktor mit einem konstanten Korrekturfaktor vereinfacht sich das anhand einer typischen Verbundbrücke dargestellte Vorgehen zur Ermüdungsbemessung von Stahlgussknoten wesentlich. Die Berechnung der zulässigen Fehlergrösse kann dadurch unabhängig vom Stahlgussknoten durchgeführt werden.

Dank der Näherungsformel kann ein Algorithmus zur Berechnung der zulässigen Anfangsfehlergrösse in Stahlgussknoten verfasst werden, mit dem eine Parameterstudie durchgeführt wird. Die 
Parameterstudie untersucht den Einfluss der Knotenabmessungen, der Materialeigenschaften und des Belastungszustands. Folgende Parameter haben einen massgebenden Einfluss auf die zulässige Anfangsgrösse von Gussfehlern:

- Bruchzähigkeit:

Die Bruchzähigkeit hat einen grossen Einfluss auf die zulässige Anfangsfehlergrösse, wenn das Bauteil nur niedriger Ausnutzung unter Ermüdung ausgesetzt ist.

- Fliessgrenze:

Mit zunehmender Fliessgrenze steigt infolge der erzielten Materialeinsparung (also infolge der reduzierten Wanddicke) die Spannungsschwingbreite an und die massgebende Fehlergrösse nimmt ab. Die Ausnutzung unter Ermüdung bleibt jedoch unverändert. Aus diesem Grund nimmt die zulässige Anfangsfehlergrösse mit zunehmender Fliessgrenze stark ab. Bei gleich bleibender Wanddicke, konstanter Ausnutzung unter Ermüdung und zunehmender Fleissgrenze nimmt die Anfangsfehlergrösse weniger stark ab.

- Wenn die Wanddicke trotz Zunahme der Fliessgrenze beibehalten wird, so hat die Fliessgrenze keinen Einfluss auf die Anfangsfehlergrösse.

Unter gewissen Umständen ist der Einfluss einiger Parameter auf die maximal zulässige Anfangsgrösse von Gussfehlern vernachlässigbar:

- Ausnutzung unter Verkehr:

Die Ausnutzung unter Verkehrslast hat bei einer hohen Spannungsschwingbreite infolge der Ermüdungslast einen vernachlässigbaren Einfluss.

$\mathrm{Ab}$ einer bestimmten Bruchzähigkeit hat die Ausnutzung unter Verkehr keinen Einfluss mehr auf die zulässige Anfangsfehlergrösse. In diesem Fall wird die massgebende Fehlergrösse nicht mehr durch das Sprödbruchkriterium bestimmt, sondern beträgt $90 \%$ der Wanddicke.

- Bruchzähigkeit:

Bei einer hohen Spannungsschwingbreite wird die zulässige Fehlergrösse nur unwesentlich von der Bruchzähigkeit beeinflusst. 


\section{ZUSAMMENFASSUNG, FOLGERUNGEN UND WEITERFÜHRENDE} ARBEITEN

\subsection{ZIELSETZUNG UND VORGEHENSWEISE}

Die Schlussfolgerungen der Arbeit werden entsprechend den im Kapitel 1 aufgeführten Zielen gegliedert. Diese sollen daher nochmals in Erinnerung gerufen werden:

1. Quantitative Erfassung des globalen Ermüdungsverhaltens von Stahlgussknoten in einem Fachwerkträger anhand experimenteller Untersuchungen an grossmassstäblichen Probekörpern.

2. Ermittlung der für eine geforderte Ermüdungsfestigkeit maximal zulässigen Anfangsgrössen von Gussfehlern in den einzelnen Bereichen eines Stahlgussknotens einer typischen Fachwerkbrücke aus Hohlprofilen in Abstimmung mit der Ermüdungsfestigkeit der Anschlussschweissnähte.

3. Entwicklung eines allgemein anwendbaren Bemessungsvorschlags für Stahlgussknoten unter Ermüdung und Bestimmung der wesentlichen Parameter.

Anhand experimenteller Untersuchungen wurde das globale Ermüdungsverhalten von Stahlgussknoten in einem Fachwerkträger quantitativ erfasst (Kapitel 3). In Abhängigkeit verschiedener Parameter wurde untersucht, inwiefern das globale Ermüdungsverhalten von Stahlgussknoten durch die zwei Komponenten ,Beanspruchbarkeit des Stahlgussknotens' und ,Beanspruchbarkeit der Schweissnahtanschlüsse' bestimmt wird. Eine wirtschaftlich optimale Bemessung eines Stahlgussknotens ist dann gegeben, wenn der Ermüdungswiderstand des Gussknotens demjenigen der Anschlussnähte entspricht. Daher wurden die maximal zulässigen Anfangsgrössen von Gussfehlern unter Berücksichtigung der Ermüdungsfestigkeit der Anschlussschweissnähte bestimmt. Dazu wurde ein numerisches Modell mit Randelementen erstellt, mit dem für im Knoten eingefügte Gussfehler Spannungsintensitätsfaktoren an der Rissfront berechnet und das Rissfortschrittsverhalten simuliert werden kann (Kapitel 4). Mit Hilfe dieses Modells wurden zunächst die für eine geforderte Ermüdungsfestigkeit maximal zulässigen Anfangsgrössen von Gussfehlern in Stahlgussknoten, die in den Längsträgern einer typischen Stahl-Beton-Verbundbrücke eingebunden sind, berechnet (Kapitel 5). Mit dem Ziel einer allgemeinen Anwendung der Ergebnisse für Stahl-BetonVerbundbrücken wurden in Kapitel 6 diese Anfangsgrössen durch eine Näherungsformel umschrieben. Dank der Näherungsformel vereinfacht sich das anhand einer typischen Rohrfachwerkbrücke dargestellte Vorgehen für die Ermüdungsbemessung von Stahlgussknoten wesentlich. Diese Näherung diente anschliessend zur Durchführung einer Parameterstudie, die den Einfluss von Materialeigenschaften wie Zähigkeit und Fliessgrenze, von verschiedenen Belastungskombinationen aus Ermüdungs- und Verkehrslast sowie der durch den Brückentyp bedingten Knotengeometrie auf die maximal zulässige Anfangsgrösse von Gussfehlern untersucht. 


\subsection{ZUSAMMENFASSUNG UND FOLGERUNGEN}

\section{Experimentelle Erfassung des Ermüdungsverhaltens von Stahlgussknoten}

Für die korrekte Erfassung der Ermüdungsbelastung der Anschlussschweissnähte und der Stahlgussknoten ist die Zusatzbelastung aus Sekundärmomenten von grosser Bedeutung. Daher wurde der Einfluss der unterschiedlichen Steifigkeiten des Gussknotens und der anschliessenden Hohlprofile auf die Spannungsverteilung im Knoten und in den Schweissnähten untersucht. Dieser Steifigkeitssprung wurde sowohl durch eine kompaktere Ausbildung der Gussknoten (kürzere Knotenstutzen und grössere Wanddicken) als auch durch die Wahl grösserer Wanddicken der Hohlprofile variiert. Die Auswertung der Messergebnisse unter statischer Belastung ergibt Folgendes:

- Die Verkürzung der Stutzenlängen hat zwei Auswirkungen. Einerseits verschiebt sich die Schweissnaht gegen den Achsenschnittpunkt zu, also in Richtung höherer Sekundärbiegemomente. Andererseits werden die Rundhohlprofile länger, ihre Biegesteifigkeit und damit die Sekundärbiegemomente nehmen ab. In den Diagonalen überwiegt der zweite Effekt, im Untergurt heben sich die Einflüsse gegenseitig auf.

- Die Reduktion der Steifigkeit der Rundhohlprofile bei gleich bleibender Stutzenlänge führt zu einer weiteren Abnahme der Sekundärbiegemomente.

Es wird daher empfohlen, bei der Wahl der Knotenform die Knotenstutzen mit minimaler Länge auszuführen. Dadurch werden die Sekundärbiegemomente in den Diagonalen reduziert und Herstellungskosten (Materialkosten) eingespart.

Die Versuchsergebnisse zeigten sehr eindeutig, dass für die in den Versuchen vorliegenden Konfigurationen die Schweissnähte für die Ermüdungsfestigkeit massgebend sind. In den Stahlgussknoten konnten keinerlei Ermüdungsrisse beobachtet werden. Nachträglich durchgeführte Ultraschalluntersuchungen und Zugversuche an aus den getesteten Stahlgussknoten ausgearbeiteten Kleinproben zeigten, dass diese eine bessere als die geforderte Gütestufe aufweisen. Auf einigen Bruchflächen der Zugproben konnten durch Sprödbruch Gussfehler freigelegt werden. Die Grösse der Fehler liegt jedoch unter der für die Gütestufe gültigen Registriergrenze für Ultraschalluntersuchungen. Die Auswertung der Resultate aus den Ermüdungsversuchen ergibt für die Schweissnähte Folgendes:

- Die Rissinitiierung fand stets an der Wurzelseite in Schweissnahtmitte statt, die Risse breiteten sich also von der Hohlprofilinnenseite her aus. Nach dem Wanddurchbruch breiteten sie sich zunächst radial entlang der Schweissnahtmitte und im weiteren Verlauf des Risswachstums entlang des Schweissnahtfusses oder entlang der Wärmeeinflusszone auf der Hohlprofilseite aus.

- Die Anzahl Lastwechsel ab dem Zeitpunkt der Rissentdeckung bis zum Abbruch des Versuchs bei einer Risslänge von ungefähr 50 \% des Hohlprofilumfangs ist im Verhältnis zur gesamten Lebensdauer sehr gering und beträgt höchstens etwa $10 \%$. Da ein Riss erst bei Wanddurchbruch visuell entdeckt werden kann, ist die Restlebensdauer bis zum Bruch der Schweissnaht sehr klein.

- Für die Stumpfnähte ohne Schweissbadsicherung ergibt sich eine Ermüdungsfestigkeit mit $\Delta \sigma_{\mathrm{C}}=54 \mathrm{MPa}$.

- Für die Stumpfnähte mit Schweissbadsicherung ergibt sich eine Ermüdungsfestigkeit mit $\Delta \sigma_{\mathrm{C}}=87 \mathrm{MPa}$. 
- Für fehlerbehaftete Nähte ohne Schweissbadsicherung wird eine Ermüdungsfestigkeit mit $\Delta \sigma_{\mathrm{C}}=27 \mathrm{MPa}$ gemessen.

Bei der Ermittlung der Kerbfälle wurden die Spannungskonzentrationen in den Schweissnähten rechnerisch mitberücksichtigt. Die Bestimmung der Ermüdungsfestigkeit beruht auf eine sehr kleine Anzahl $(<10)$ Versuchsergebnisse. Aus diesem Grund sind weitere Versuchsergebnisse erforderlich.

Die Versuchsergebnisse haben sehr eindeutig gezeigt, dass für die vorliegende Konfiguration der Versuchskörper die Stumpfschweissnähte für die globale Ermüdungsfestigkeit massgebend sind. Weil für eine wirtschaftlich optimal Bemessung der Stahlgussknoten ihr Ermüdungswiderstand demjenigen der Schweissnahtanschlüsse entsprechen soll, haben sich die weiteren numerischen und analytischen Untersuchungen auf die Ermüdungsfestigkeit der Stahlgussknoten konzentriert. Der Einfluss der Ausbildung der Stumpfschweissnähte auf die Ermüdungsfestigkeit wird zeitgleich mit der vorliegenden Arbeit im Rahmen eines gemeinsamen Forschungsprojekts ${ }^{7}$ mit der Versuchanstalt für Stahl, Holz und Steine der Universität Karlsruhe, welches Ende 2006 abgeschlossen sein wird, eingehender untersucht.

\section{Maximal zulässige Anfangsgrössen von Gussfehlern in Stahlgussknoten einer typischen Fach- werkbrücke}

Die maximal zulässigen Anfangsgrössen von Gussfehlern in Stahlgussknoten einer typischen StahlBeton-Verbundbrücke wurden mit Hilfe eines numerischen Modells mit Randelementen für eine geforderte Ermüdungsfestigkeit berechnet. Dazu wurden für zwei unterschiedlich belastete Knoten die Einwirkungen sowohl infolge statischer Last als auch infolge normierter Ermüdungslast bestimmt. Mit dem numerischen Modell wurden die Spannungsintensitätsfaktoren an der Rissfront berechnet und das Risswachstum simuliert. Folgendes kann aus den numerischen Untersuchungen abgeleitet werden:

- Sprödbruch des rissbehafteten Knotens kann für die untersuchte Situation ausgeschlossen werden. Die Spannungsintensitätsfaktoren infolge statischer Last lagen trotz der sehr konservativen Annahme der Verkehrslast als Leiteinwirkung für den Sprödbruchnachweis an allen untersuchten Fehlerstellen unterhalb der in der Norm aufgeführten Bruchzähigkeit des Stahlgusses von $K_{\mathrm{Ic}}=2400 \mathrm{~N} \cdot \mathrm{mm}^{-3 / 2}$ und die Hauptspannungen im Knoten überstiegen $60 \%$ der Fliessgrenze nicht. Als Bruchkriterium wurde daher eine Risstiefe von $90 \%$ der Wanddicke an der Fehlerstelle festgelegt, da ein Wanddurchbruch des Risses als nicht tolerierbar eingestuft wurde.

- Es wurde eine Reihe konservativer Annahmen zur Berechnung der maximal zulässigen Anfangsfehlergrössen verwendet: Simulation der Gussfehler als Oberflächenrisse, Sprödbruchnachweis mit der Bruchzähigkeit $K_{\text {Ic }}$ (für ebenen Dehnungszustand) und den Maximalwerten der Spannungsintensitätsfaktoren am sich auf der Oberfläche befindlichen Rissrand, Vernachlässigung des Risswachstums nach dem Wanddurchbruch und Marge von $10 \%$ der Wanddicke vor dem Wanddurchbruch. Trotz dieser konservativen Annahmen sind die zulässigen Anfangsfehlergrössen im Verhältnis zur Wanddicke durchwegs sehr gross. Die Spanne der zulässigen Fehlergrössen reicht von 28 bis 88 \% der Wanddicke. Für die Diagonalstutzen ergeben sich die

\footnotetext{
${ }^{7}$ Forschungsvorhaben der FOSTA-Forschungsvereinigung Stahlanwendung e.V. P591 „Wirtschaftliches Bauen von Strassen- und Eisenbahnbrücken mit Stahlhohlprofilen“
} 
kleinsten zulässigen Anfangsfehler beim Auflager, für die Untergurtstutzen in Feldmitte der Fachwerkbrücke.

- Wird der kleinste zulässige Oberflächenfehler von $28 \%$ der Wanddicke durch zwei Oberflächenfehler und einen Innenfehler in der Kernzone ersetzt (analog zu den Fehlerbeschreibungen in der Liefernorm [DIN 1690-2:1985] und nach [BS 7910:1999]), entsprechen deren maximal zulässige Fehlergrössen mit 10 bzw. 17 \% der Wanddicke in etwa den in der Norm festgelegten Grenzwert für die Gütestufe 4 von maximal 10 \% (Randzone) bzw. 15 \% (Kernzone) der Wanddicke.

\section{Vorgehen für die Ermüdungsbemessung und Parameterstudie}

Durch die Näherungsformel für den Spannungsintensitätsfaktor mit einem konstanten Korrekturfaktor vereinfacht sich das anhand einer typischen Verbundbrücke dargestellte Vorgehen zur Ermüdungsbemessung von Stahlgussknoten wesentlich. Die Berechnungen der maximal zulässigen Fehlergrössen können dadurch unabhängig vom Stahlgussknoten durchgeführt werden. Es wurde gezeigt, dass durch die Einführung dieser Näherungsformel eine gute und einfache Abschätzung der maximal zulässigen Anfangsgrösse von Gussfehlern in Stahlgussknoten möglich ist, ohne dass zeitaufwendige numerische Rissfortschrittssimulationen durchgeführt werden müssen. Die Untersuchungen zeigen Folgendes:

- Für Oberflächenrisse muss nicht nach Lage der Risse auf der Aussen- oder Innenseite der Gussknoten unterschieden werden, da sich die Spannungsintensitätsfaktoren nicht wesentlich unterschieden.

- Der Korrekturfaktor für den Spannungsintensitätsfaktor von Oberflächenrissen (mit $a / c=1.0$ ) im Stahlgussknoten beträgt für die Risstiefe $Y_{\mathrm{a}}=0.67$ und für den Rissrand $Y_{\mathrm{c}}=0.77$.

- Der Korrekturfaktor für den Spannungsintensitätsfaktor von Innenrissen (mit $a / c=1.0$ ) im Stahlgussknoten beträgt entlang der Rissfront $Y=0.64$. Dieser Wert entspricht dem Korrekturfaktor aus der Literatur für kreisförmige Innenrisse.

- Die Untersuchungen zeigen, dass eine vereinfachte, näherungsweise Berechnung des Spannungsintensitätsfaktors $K_{\mathrm{I}}$ mit einem konstanten Korrekturfaktor $Y$ möglich ist. Der Vergleich zwischen den berechneten zulässigen Anfangsfehlergrössen aus dem numerischen Modell und denjenigen aus der Näherungsformel zeigt im Allgemeinen Abweichungen von deutlich unter $\pm 20 \%$.

Die Parameterstudie untersucht den Einfluss der Knotenabmessungen, der Materialeigenschaften und des Belastungszustands. Der Belastungszustand ist durch die Ausnutzung unter Verkehr (für den Sprödbruchnachweis mit der Bauteiltemperatur als Leiteinwirkung und der Verkehrslast als Begleiteinwirkung) und der Ausnutzung unter Ermüdung gegeben. Folgende Parameter haben einen massgebenden Einfluss auf die zulässige Anfangsgrösse von Gussfehlern:

- Bruchzähigkeit:

Die Bruchzähigkeit hat einen grossen Einfluss auf die zulässige Anfangsfehlergrösse, wenn das Bauteil nur niedriger Ausnutzung unter Ermüdung ausgesetzt ist.

- Fliessgrenze:

Mit zunehmender Fliessgrenze steigt infolge der erzielten Materialeinsparung (also infolge der reduzierten Wanddicke) die Spannungsschwingbreite an und die massgebende Fehlergrösse 
nimmt ab. Die Ausnutzung unter Ermüdung bleibt jedoch unverändert. Aus diesem Grund nimmt die zulässige Anfangsfehlergrösse mit zunehmender Fliessgrenze stark ab. Bei gleich bleibender Wanddicke, konstanter Ausnutzung unter Ermüdung und zunehmender Fleissgrenze nimmt die Anfangsfehlergrösse weniger stark ab.

Unter gewissen Umständen ist der Einfluss einiger Parameter auf die maximal zulässige Anfangsgrösse von Gussfehlern vernachlässigbar:

- Ausnutzung unter Verkehr:

Die Ausnutzung unter Verkehrslast hat bei einer hohen Spannungsschwingbreite infolge der Ermüdungslast einen vernachlässigbaren Einfluss.

$\mathrm{Ab}$ einer bestimmten Bruchzähigkeit hat die Ausnutzung unter Verkehr keinen Einfluss mehr auf die zulässige Anfangsfehlergrösse. In diesem Fall wird die massgebende Fehlergrösse nicht mehr durch das Sprödbruchkriterium bestimmt, sondern beträgt 90 \% der Wanddicke.

- Bruchzähigkeit:

Bei einer hohen Spannungsschwingbreite wird die zulässige Fehlergrösse nur unwesentlich von der Bruchzähigkeit beeinflusst.

\subsection{SCHLUSSFOLGERUNGEN}

Die Anschlussschweissnähte der Stahlgussknoten können als Stumpfschweissnähte mit oder ohne Schweissbadsicherung ausgebildet werden. Durch die Schweissbadsicherung kann eine gute Aufschmelzung der Nahtflanken im Wurzelbereich und somit eine bessere Qualität der Schweissnahtwurzel garantiert werden. In der Wurzellage ist aber ein Aufschmelzen der Badsicherung unvermeidlich. Dadurch wird an der Schweissnahtwurzel eine signifikante Kerbe eingefügt. Die Versuchsergebnisse zeigten jedoch, dass trotz der vorhandenen Kerbe die Ermüdungsfestigkeit der Stumpfnähte mit Schweissbadsicherung höher ausfällt $\left(\Delta \sigma_{\mathrm{C}}=87 \mathrm{MPa}\right)$ als diejenige für Stumpfnähte ohne Schweissbadsicherung $\left(\Delta \sigma_{\mathrm{C}}=54 \mathrm{MPa}\right)$. Für die Anwendung der Kerbfälle muss die Spannungskonzentration in den Schweissnähten mitberücksichtigt werden.

Basierend auf bestehenden Stahl-Beton-Verbundbrücken mit Rohrfachwerken als Hauptlängsträger und Stahlgussknoten als Fachwerkknoten wurde eine Brücke mit typischen Abmessungen definiert. Für dieses Brückenmodell wurden anhand numerischer Untersuchungen für eine geforderte Ermüdungsfestigkeit maximal zulässige Anfangsgrössen von Gussfehlern berechnet. Für die verschiedenen Bereiche in zwei unterschiedlich belasteten Stahlgussknoten lagen die maximal zulässigen Fehlergrössen in einer Spannweite von knapp 30 bis knapp 90 \% der Wanddicke an der Fehlerstelle. Für die Diagonalstutzen ergeben sich die kleinsten zulässigen Anfangsfehler beim Auflager, für die Untergurtstutzen in Feldmitte der Fachwerkbrücke.

Bei der Herstellung von Stahlgusskomponenten einer bestimmten Gütestufe wird in der Regel nicht der Herstellungsprozess reguliert. Die Gütestufe wird nicht auf Bestellung hergestellt sondern durch entsprechende zerstörungsfreie Prüfungen nachgewiesen. Je besser die Gütestufe umso höher ist der Prüf- und der damit verbundene Kostenaufwand. Durch die Angabe der maximal zulässigen Anfangsgrössen von Gussfehlern für eine geforderte Ermüdungsfestigkeit können die Kosten für zerstörungsfreie Prüfungen reduziert werden. Andererseits ist es wahrscheinlich, dass die Stahlgussknoten viel kleinere Gussfehler (und damit eine höhere Ermüdungsfestigkeit) aufweisen als 
durch Prüfung nachgewiesen wurde. Erfahrungen aus den experimentellen Untersuchungen zeigen, dass die Qualität der Stahlgussknoten weit besser war, als bei der Bestellung mit der Stahlgiesserei vereinbart wurde. Dies erklärt die Tatsache, dass für die in den Versuchen vorliegenden Konfigurationen die Schweissnähte für die globale Ermüdungsfestigkeit der Knoten massgebend waren. Um die tatsächlich vorhandene Ermüdungsfestigkeit der Stahlgussknoten ausnützen zu können, muss sie mit zerstörungsfreier Prüfung garantiert und die Ermüdungsfestigkeit der Anschlussschweissnähte wesentlich verbessert werden.

Durch die Einführung einer auf einem konstanten Korrekturfaktor beruhenden Näherungsformel für den Spannungsintensitätsfaktor von Gussfehlern, die als Oberflächen- oder Innenrisse angenommen werden, ist eine gute und einfache Abschätzung der maximal zulässigen Anfangsgrösse von Gussfehlern in Stahlgussknoten möglich. Die Berechnung der Anfangsfehlergrössen kann als Algorithmus implementiert werden, beispielsweise mit Hilfe der Programmiersprache Visual Basic for Applications (VBA) als benutzerdefinierte Funktion für Microsoft Excel. Dank der Näherungsformel vereinfacht sich das anhand einer typischen Rohrfachwerkbrücke dargestellte Bemessungsvorgehen für den Ermüdungswiderstand von Stahlgussknoten wesentlich.

Die Wahl des Materials, der Geometrie von Stahlgussknoten und der Ausnutzung unter Verkehrslast und Ermüdungslast können einen unterschiedlich grossen Einfluss auf die maximal zulässigen Anfangsgrössen von Gussfehlern haben. Es wurde gezeigt, dass der Einfluss der Ausnutzung unter Verkehr mit Zunahme der Spannungsschwingbreite infolge der Ermüdungslast abnimmt. Bei hohen Spannungsschwingbreiten hat die Bruchzähigkeit des Stahlgusses keinen Einfluss. Bei hoher Bruchzähigkeit wird die zulässige Anfangsfehlergrösse nur durch die Spannungsschwingbreite beeinflusst und ist unabhängig von der Ausnutzung unter Verkehr.

\subsection{WEITERFÜHRENDE ARBEITEN}

In dieser Arbeit wird ein Konzept zur Abschätzung der für eine geforderte Ermüdungsfestigkeit maximal zulässigen Anfangsgrössen von Gussfehlern in Stahlgussknoten vorgeschlagen. Sämtliche Berechnungen wurden deterministisch durchgeführt, auf probabilistische Betrachtungen wurde im Rahmen dieser Arbeit verzichtet. Für die Einwirkungen wurden die charakteristischen Werte mit den dazugehörigen Lastbeiwerten nach der Norm [SIA 261] angesetzt. Für die Materialkennwerte des Stahlgusses wurden die charakteristischen Werte für den GS-20 Mn 5 nach [DIN 17182:1992] herangezogen. Für die Rissfortschrittsparameter $C$ und $m$ wurden diejenigen für ferritisch-perlitische Stähle angesetzt. Dieses Konzept gilt es in einem nächsten Schritt in ein probabilistisches Sicherheitskonzept zu integrieren. Insbesondere ist es interessant, den Einfluss des realen Verkehraufkommens im Vergleich zu den Lastmodellen aus der Norm auf die maximal zulässigen Anfangsgrössen von Gussfehlern zu untersuchen. Ein weiterer Aspekt ist die Verteilung der Gussfehler im Stahlgussknoten. Auch die Rissfortschrittsparameter $C$ und $m$ müssen für verschiedene Stahlgusssorten, die im Brückenbau heute bereits eingesetzt werden oder in Zukunft eingesetzt werden könnten, experimentell bestimmt werden.

Mit den heute in Europa gültigen Normen für Stahlgusskomponenten muss der Ingenieur zwischen den berechneten maximal zulässigen Anfangsgrössen der Gussfehler und den Gütestufen der Normen einen Zusammenhang herstellen. Die Detektierbarkeit der Fehler ist dabei zu berücksichtigen. 
Zur Ermüdungsfestigkeit der Stumpfschweissnaht zwischen Rundhohlprofilen liefert die Norm [prEN 1993-1-9:2003] nur sehr wenig Information. Einerseits beschränken sich die Angaben auf Fälle ohne Schweissbadsicherung und ohne exzentrische Anschlüsse der Rundhohlprofile, andererseits sind sie auf Grund des sehr eingeschränkten zugelassenen Wanddickenbereichs nicht für den Brückenbau anwendbar. Da für die Anwendung von Stahlgussknoten im Brückenbau die Stumpfschweissnähte zwischen den Knotenstutzen und den Hohlprofilen jedoch von zentraler Bedeutung sind und die vorgeschlagenen Kerbfälle auf sehr wenig Versuchsresultate basieren, besteht auf diesem Gebiet noch ein grosser Forschungsbedarf. Ein wichtiges Ziel ist die Verbesserung der Ermüdungsfestigkeit der Schweissnaht, damit die gute Ermüdungsfestigkeit der Stahlgussknoten ausgenutzt werden kann. Anhand experimenteller Untersuchungen muss die Ermüdungsfestigkeit von Stumpfschweissnähten zwischen Rundhohlprofilen untersucht werden. Dabei soll der Einfluss der Schweissbadsicherung und ihrer Ausgestaltung, beispielsweise als Blechstreifen, Keramikring oder angegossene Nase im Gussteil, systematisch untersucht werden. Ebenfalls eingehender untersucht werden muss der Einfluss des Wanddickensprungs in der Schweissnaht und derjenige der Wanddicke. Es ist zudem von Interesse, mittels analytischer und numerischer Untersuchungen den Einfluss von exzentrischen Anschlüssen (durch verschiedene Wanddicken bei gleichem Aussenradius der Hohlprofile, durch verschiedene Radien bei gleicher Wanddicke der Hohlprofile, durch ovale Kreisquerschnitte oder durch Verschiebung eines Kreisquerschnitts in Bezug auf die Achse des anderen) auf die Spannungskonzentration in der Schweissnaht zu erforschen und mit bestehenden Literaturangaben zu vergleichen. 



\section{LITERATURVERZEICHNIS}

[ABAQUS 2000]

[Ackermann 1999]

[ASTM E 186-98]

[ASTM E 1921-03 2003]

[ASTM E 280-98]

[ASTM E 446-98]

[Barsom 1971]

[Barsom et al. 1970]

[Barsom et al. 1999]

[BEASY 2003]

[Beeley 2001]

[Bergmann 1995]

[Bergmann et al. 1981]

[Bernhardt et al. 2003]
ABAQUS, Standard version 6.0.1 (computer software), Hibitt, Karlson \& Sorenson (HKS), Inc., Pawtucket, Rhode Island, U.S.A., 2000.

ACKERMANN, P., Halle 13, Deutsche Messe AG, Expo 2000, Hannover GmbH, Prestel Verlag, 1999.

Standard reference radiographs for heavy-walled (2 to 4 1/2-in. (51 to 114$\mathrm{mm}$ )) steel castings, ASTM E 186-98, American Society for Testing Materials, 1998.

ASTM E 1921-03, Standard test method for determination of reference temperature, T0, for ferritic steels in the transition range, November 2003.

Standard reference radiographs for heavy-walled (4 1/2 to 12 -in. (114 to 305mm)) steel castings, ASTM E 280-98, American Society for Testing Materials, 1998.

Standard reference radiographs for steel castings up to $2 \mathrm{in}$. (51mm) in thickness, ASTM E 446-98, American Society for Testing Materials, USA, 1998.

BARSOM, J. M., Fatigue-crack propagation in steels of various yield strengths, Vol. 93, No. 4, Transactions of the ASME, Journal of engineering for industry, Serie B, November 1971.

BARSOM, J.M. AND ROLFE, S.T., Correlations Between KIc and Charpy VNotch Test Results in the Transition Temperature Range, Impact Testing of Metals, ASTM STP 466, pp. 281-302, ASTM (American Society for Testing and Materials), 1970.

BARSOM, J. M., ROLFE, S. T., Fracture and fatigue control in structures: application of fracture mechanics, Third edition, Prentice-Hall, New Jersey, 1999.

BEASY User Guide, Computational mechanics BEASY Ltd, Ashurst, Southhampton, UK, 2003.

BEELEY, P., Foundry technology, 2001.

BERGMANN, M., Stress intensity factors for circumferential surface cracks in pipes, Fatigue \& fracture of engineering materials \& structures, Vol. 18, No. 10, pp. 1155-1172, 1995.

BERGMANN, J., BISWAS, K., HOFF, H.-H., SEEGER, T., Fatigue behaviour of HOESCH Gs Ark 10, Part 1: Experimental results, Fachgebiet Werkstoffmechanik, Technische Hochschule Darmstadt, Report CSN 6, October 1981.

BERNHARDT, K., MOHR, B., SEIFRIED, G., ANGELMAIER, V., Talbrücke Korntal-Münchingen - innovativer Brückenentwurf als RohrfachwerkVerbundbrücke, No. 2, pp. 61-70, Stahlbau 72, 2003. 
[Blair et al. 1995]

[Blumenauer 1993]

[Broughton et al. 1997]

[BS 7608:1993]

[BS 7910:1999]

[Buberl et al. 1995]

[Christianus 1998]

[Christianus et al. 1998]

[CIDECT 1991]

[Cisilino et al. 2004]

[Connelly et al. 1993]

[Dahl et al. 1995]

[Dauner 1998]

[Dauner et al. 1998]
BLAIR, M., STEVENS, T. L., Steel castings handbook, Steel founders' Society of America and ASM International, 1995.

BLUMENAUER, H., Technische Bruchmechanik, Deutscher Verlag für Grundstoffindustrie, Leipzig, Germany, 1993.

BROUGHTON, P. HAYES, R., WOOD, A., Cast steel nodes for the ekofisk 2/4J jacket, Proceedings of the Institution of Civil Engineers Structures and buildings, Vol. 122, pp. 266-280, Paper 11248, October 1997.

BRITISH STANDARD INSTITUTE BSI, Code for practice for fatigue design and assessment of steel structures, BS 7608:1993, British Standard BSI, 1993.

BRITISH STANDARD INSTITUTE BSI, Guide on methods for accessing the acceptability of flaws in metallic structures, British standard, 2000.

BUBERL, A., SCHUSTER, F., HANUS, R., Kosteneinfluss - Qualitätsgrad von Stahlguss, Giesserei-Rundschau, Vol. 42, No. 3/4, pp. 14-20, 1995.

CHRISTIANUS, D., Erprobung von höherfesten StahlgussverbundRohrknoten in bauteilähnlichen Massstab zum Einsatz in OffshoreBauwerken, konstruieren+giessen, Vol. 23, No. 2, pp. 4-13, 1998.

CHRISTIANUS, D., HERFURTH, K., Europäische Normung: Prüfung der Oberflächenrauheit mit Hilfe von Vergleichsmustern - DIN EN 1370, konstruieren + giessen, Vol. 23, No. 4, pp. 26-30, 1998.

CIDECT, Background document: Eurocode 3: Chapter 9, Document 9.03, Part 1: Classification method - Statistical evaluation, CIDECT-Document 7M- 1/91, 1991.

CISILINO, A.P., ALIABADI M.H., Dual boundary element assessment of three-dimensional fatigue crack growth, Engineering Analysis with Boundary Elements, pp. 1157-1173, 2004.

CONNELLY, L. M., ZETTLEMOYER, N., Stress concentration at girth welds of tubulars with axial wall misalignment, Tubular Structures V, Edited by M.G. Coutie and G. Davies, pp. 309-322, 1993.

DAHL, W., LANGENBERG, P., CHRISTIANUS, D., MÖLL, R., SEEGER, T., AMSTUTZ, H., KLEE, S., Verwendung von Stahlguss GS-13 MnNi 64 für den Neubau der Kronprinzenbrücke in Berlin, FG-122/1995, Fachgebiet Werkstoffmechanik Technische Hochschule Darmstadt, 1995.

DAUNER, H.-G., Der Viadukt von Lully, Eine Neuheit im Verbundbrückenbau, No. 1, pp. 1-14, Stahlbau 67, 1998.

DAUNER, H.-G., DECORGES, G., ORIBASI, A., WÉRY, D., The Lully Viaduct, a composite bridge with steel tube truss, Journal of Constructional Steel Research, paper no 55, Elsevier, 1998. 
[Deutsche Bundesbahn 1993] DEUTSCHE BUNDESBAHN, DS - 804: Vorschriften für Eisenbahnbrücken und sonstige Ingenieurbauwerke (VEI), Fassung 1.6.93, Bekanntgabe B3, 1993.

[DIN 1690-2:1985]

[DIN 17182:1992]

[DIN 17205-1: 1998]

[DIN 54111-2:1982]

[DIN EN 1370:1996]

[DNV 2001]

[ECCS-CECM-EKS 1987]

[Edwards et al. 1981]

[Edwards et al. 1985]

[Ellis et al. 1983]

Technische Lieferbedingungen für Gusstücke aus metallischen Werkstoffen, DIN 1690, Teil 2: 1985, DIN, June 1985.

Stahlgusssorten mit verbesserter Schweisseignung und Zähigkeit für allgemeine Verwendungszwecke, DIN 17182:1992, Ausgabe 1992-05, DIN, 1992.

Stahlguss für das Bauwesen und für allgemeine Anwendungszwecke, Teil 1: Allgemeines, DIN 17205, Teil 1, DIN, April 1998.

Zerstörungsfreie Prüfung; Prüfung metallischer Werkstoffe mit Röntgen- und Gammastrahlen; Aufnahme von Durchstrahlungsbildern von Gussstücken aus Eisenwerkstoffen, DIN 54111, Teil 2: 1982, DIN, June 1982.

Giessereiwesen - Prüfung der Oberflächenrauheit mit Hilfe von Vergleichsmustern, DIN EN 1370:1996, DIN, February 1997.

DNV, Fatigue strength analysis of offshore steel structures, Recommended practice RP-C203, Det Norske Veritas, October 2001.

Empfehlungen für die Bemessung und Konstruktion von ermüdungsbeanspruchten Stahlbauten, Technical Commitee 6 - Fatigue / European Convention for Constructional Steelwork, Brussels, No. 43, ECCS-CECM-EKS, 1987.

EDWARDS, C. D., FESSLER, H., Design and stress analysis of a light, cast $90^{\circ}-45^{\circ}$ K-joint, Steel in marine structures, No. TS4.4, October 1981.

EDWARDS, C. D., FESSLER, H., Stress concentrations in cast corner joints of the tubular structures, International conference on behaviour of offshore structures, Delft, 1985.

ELLIS, N., SALAMA, M. M., BEGGS, D. V., Evaluation of structural steel castings for the Hutton Tension Leg Platform, Offshore technology conference, No. OTC 4450, pp. 65-68, 1983.

[EN 1011-2:2001]

[EN 10204:1991/A1:1995]

[Fricke et al. 2004]

Empfehlungen zum Schweissen metallischer Werkstoffe. Teil 2: Lichtbogenschweissen von ferritischen Stählen, EN 1011-2:2001, DIN, January 2001.

Arten von Prüfbescheinigungen, EN 10204:1991/A1:1995, DIN, 1995.

FRICKE, W., DOERK, O., Fatigue analysis of fillet welds around stiffener and bracket toes, Doc. XIII-2034-04, XV-1170-04, International Institute of Welding (IIW), Cambridge: Abington, May 2004.

[FWH 2004]

FWH, Auszug aus Prüfprotokollen zur Ermittlung der Kerbschlagarbeit für den Stahlguss GS-20 Mn 5 V, Interne Prüfprotokolle, Friedrich WilhelmsHütte GmbH, 2004. 
[Glijnis et al. 2003]

[Gysel et al. 1984]

[Gysel et al. 1984.1]

[Haneke et al. 1982]

[Hartmann 1989]

[Heuler et al. 1991]

[Heuler et al. 1991.1]

[Heuler et al. 1991.2]

[Hirt et al. 1998]

[IfG - DK 2005]

[ISO 4987:1992]

[IWT 2004]

[Knipp 1961]
GLIJNIS, P. C., CROMMENTUYEN, J., To cast or not to cast, Proceedings of the 10th International Symposium on Tubular Structures, pp. 12-134, Tubular Structures X, A.A. Balkema Publishers, Madrid, 2003.

GYSEL, W., BÄCHTOLD, H., BINDA, B., BRUGNONI, E., Sicherheit hochbeanspruchter Stahlguss-Bauteile, beurteilt nach dem heutigen Stand der Herstell- und Prüftechnologie - Teil 1, Giesserei 71, No. 6, pp. 246-251, March 1984.

GYSEL, W., BÄCHTOLD, H., BINDA, B., BRUGNONI, E., Sicherheit hochbeanspruchter Stahlguss-Bauteile, beurteilt nach dem heutigen Stand der Herstell- und Prüftechnologie - Teil 2, Giesserei 71, No. 8, pp. 325-332, April 1984.

HANEKE, M., GANTKE, F., Research into material for cast steel nodes, Second international conference on offshore welded structures, No. 49, London, November 1982.

HARTMANN, F., Introduction to boundary elements, Springer-Verlag, 1989.

HEULER, P., BERGER, C., MOTZ, J. M., Oberflächennahe Fehlstellen im Stahlguss, Bewertung mit Hilfe von Kerb- und Bruchmechanikkonzepten Teil 1: Fehlstellen und ihre Wirkung, Versuchskonzept, Materialprüfung 33, Vol. 9, pp. 246-252, 1991.

HEUlER, P., BERGER, C., MOTZ, J. M., Oberflächennahe Fehlstellen im Stahlguss, Bewertung mit Hilfe von Kerb- und Bruchmechanikkonzepten, Teil 2: Rechnerische Bewertung von Fehlstellen, Materialprüfung 33, Vol. 10, pp. 292-296, 1991.

HEUlER, P., BERGER, C., MOTZ, J. M., Oberflächennahe Fehlstellen im Stahlguss, Bewertung mit Hilfe von Kerb- und Bruchmechanikkonzepten, Teil 3: Bruchmechanisches Verhalten von Warmrissen, Zusammenfassung, Materialprüfung 33, Vol. 11-12, pp. 329-331, 1991.

HIRT, M. A., BEZ, R., Stahlbau: Grundbegriffe und Bemessungsverfahren, Ernst \& Sohn, Berlin, 1998.

IFG - DK, Gussfehleratlas, http://www.roheisen.de/Gussfehlerat-las/, Institut für Giessereitechnik und DK Recycling und Roheisen GmbH, 2005.

Steel castings - Penetrant inspection, ISO 4987, First edition 1992-06-01, ISO, 1992.

IWT, Prüfbericht: Erstellung einer Masterkurve nach ASTM E 1921-02 für Stahlguss, Ingenieurbüro für Werkstofftechnik, 2004.

KNIPP, E., Fehlererscheinungen an Gussstücken, Giesserei-Verlag GmbH, Düsseldorf, 1961. 
[Koivula et al. 1989]

[Kuhlmann et al. 2002]

[Kühn 2005]

[Lampman et al. 1996]

[Lotsberg 1998]

[Ma et al. 1997]

[Mang et al. 1999]

[Mang et al. 2001]

[McClintock et al. 1965]

[Mellings et al. 2005]

[Mi et al. 1992]

[Mi et al. 1994]

[Microsoft 2003]

[Milne et al. 1986]
KOIVULA, J., KATILA, R., LIIMATAINEN, J., MARTIKAINEN, H., Weldability of high purity offshore cast steel, Ironmaking and Steelmaking, Vol. 16, No. 1, pp. 41-48, 1989.

KUHLMANN, U., GÜNTHER, H.-P., SAUL, R., HÄDERLE, M.-U., STUBA, G., Zur Anwendung geschweisster Hohlprofilknoten im Brückenbau, Stahlbau, pp. 507-515, Ernst \& Sohn, 2002.

KÜHN, B., Beitrag zur Vereinheitlichung der europäischen Regelungen zur Vermeidung von Sprödbruch, Lehrstuhl für Stahlbau, RWTH Aachen, 2005.

LAMPMAN, S., R., DAVIDSON, G., M., REIDENBACH, F., BORING, R., L., HAMMEL, A., HENRY, S., D., SCOTT, W., W., ASM Handbook: Fatigue and fracture, ASM International, Vol. 19, 1996.

LOTSBERG, I., Stress concentration factors at circumferential welds in tubulars, Marine Structures, Vol. 11, pp. 207-230, 1998.

MA, A., SHARP, J. V., Fatigue design of cast steel nodes in offshore structures based on research data, No. 124, pp. 112-126, Proceeding of the institution of civil engineers: water, maritime \& energy, June 1997.

MANG, F., HERION, S., On the Fatigue Behaviour of Welded Cast Steel Steel Connections, Int. Conf. on Current and Future Trends in Bridge Design, Construction and Maintenance, Singapore, 1999.

MANG, F., HERION, S., Guss im Bauwesen, Stahlbau Kalender 2001, pp. 625-667, Ernst \& Sohn, 2001.

MCCLINTOCK, F. A., IRWIN, G. R., Plasticity aspects of fracture mechanics, American society for testing and materials, No. STP 381, Philadelphia, 1965.

MELLINGS, S., BAYNHAM, J., ADEY, R. A., Automatic crack growth prediction in rails with BEM, Engineering Fracture Mechanics, pp. 309-318, 2005 .

MI, Y., ALAIBADI, M. H., Dual boundary element method for three dimensional fracture mechanics analysis, Engineering analysis with boundary elements, Vol. 10, pp. 161-171, 1992.

MI, Y., ALIABADI, M. H., Three-dimensional crack growth simulation using BEM, Computers \& Structures, Vol. 52, pp. 871-878, 1994.

MICROSOFT, Microsoft Office Excel, Microsoft corporation, one Microsoft way, Redmond, WA, U.S.A., 2003.

MILNE, I., AINSWORTH, R.A., DOWLING, A.R., STEWART, A.T., Assessment of the integrity of structures containing defects, R/H/R6 - Revision 3, Central Electricity Generating Board, May 1986. 
[Murakami et al. 1994]

[N.D.T. SA 2004]

[N.D.T. SA 2004.1]

[Nakamura et al. 1987]

[Newmann et al. 1986]

[Ohba et al. 1979]

[OTH 92 390:1999]

[Paris 1960]

[Peeker 1997]

[Portela et al. 1993]

[prEN 1993-1-10:2003]

[prEN 1993-1-9:2003]

[Radaj 1995]

[Richardson 1996]

[Richter et al. 2001]
MURAKAMI, Y., ENDO, M., Effects of defects, inclusions and inhomogeneities on fatigue strength, International journal of fatigue : structural integrity durability - damage tolerance, Vol. 16, pp. 163-182, April 1994.

N.D.T. SA, Rapport de contrôle sur nœuds moulés, No. 2854 b, April 2004.

N.D.T. SA, Rapport complémentaire de contrôle sur nœuds moulés, No. 2854 b compl., June 2004.

NAKAMURA, K., SADA, H., NISHIMURA, H., MORIYAMA, H., Fatigue strength of cast steel nodes, Safety criteria in design of tubular structures, Proceedings of the international meeting in Tokyo, Japan, 1987.

NEWMANN, J. C., RAJU, I. S., Stress-Intensity Factor Equations For Cracks In Three-Dimensional Finite Bodies Subjected To Tension And Bending Loads, Computational methods in the mechanics of fracture, pp. 311-334, Elsevier Science, 1986.

OHBA, H., SUSEI, S., SAKAI, T., ATSUTA, T., OHKUMA, Y., OHMINAMI, R., TAMURA, A., The development of castings leg nodes for a jack-up rig, Offshore technology conference, No. OTC 3426, pp. 601-604, 1979.

HEALTH AND SAFETY EXECUTIVE, Background to new fatigue guidance for steel joints and connections in offshore structures, Offshore Technology Report OTH 92 390, Health and Safety Executive, December 1999.

PARIS, P. C., The growth of cracks due to variations in load, PhD. Dissertation, Lehigh University, Bethlehem, Pennsylvania, U.S.A., 1960.

PEEKER, E., Extended numerical modelling of fatigue behavior, $\mathrm{PhD}$ thesis EPFL n ${ }^{\circ} 1617$, Swiss Federal Institute of Technology (EPFL), Lausanne, 1997.

PORTElA, A., AliABADI, M. H., ROOKE, D. P., Dual boundary element incremental analysis of crack propagation, Computers \& Structures, Vol. 46, pp. 237-247, 1993.

Eurocode 3: Design of steel structures - Part 1-10: Material toughness and through-thickness properties, prEN 1993-1-10:2003, CEN, November 2003.

Eurocode 3: Design of steel structure - Part 1.9: Fatigue strength of steel structures, prEN 1993-1-9:2003, CEN, November 2003.

RADAJ, D., Ermüdungsfestigkeit, Springer-Verlag, 1995.

RICHARDSON, R.C., Higher strength cast steel for offshore structures, World Expro - Marine and subsea, pp. 161-164, BLDSC Shelfmark, 1996.

RICHTER, CH., SEEGER, T., AMSTUTZ, H., KLEE, S., DAHL, W., LANGENBERG, P., CHRISTIANUS, D., MÖLL, R., Bruchmechanischer Festigkeitsnachweis für die Stahlgusselemente der Kronprinzenbrücke in Berlin, Stahlbau 70 (2001), No. 4, pp. 251-257, Ernst \& Sohn Verlag, 2001. 
[RSTAB 2003]

[Sailors et al. 1972]

[Schlaich et al. 1999]

[Schlaich et al. 1999.1]

[Schlaich et al. 1999.2]

[Schlaich et al. 2000.1]

[Schober 2000]

[Schumacher 2003]

[Schumacher et al. 2003]

[SDRC 1999]

[Sedlacek et al. 2003.1]

[Seeger 1981]

[Seifried et al. 1999]

[SIA 161/1]
RSTAB, Version 5.11, Ing.-Software Dlubal GmbH, Tiefenbach, Germany, 2003.

SAILORS, R. H., CORTEN, H. T., Relationship between material fracture toughness using fracture mechanics and transition temperature tests, Proceedings of the 1971 fifth National symposium on fracture mechanics : Urbana-Champaign - Ill., 31 Aug.-2 Sept.1971, ASTM STP 514, No. Part II, pp. 164-191, 1972.

SCHLAICH, J., SCHOBER, H., Rohrknoten aus Stahlguss, Stahlbau, Vol. 68, No. 8, pp. 652-665, Ernst \& Sohn, 1999.

SCHLAICH, J., SCHOBER, H., Rohrknoten aus Stahlguss (Fortsetzung aus Heft 8/99 und Schluss), Stahlbau, Vol. 68, No. 8, pp. 734-752, Ernst \& Sohn, 1999.

SCHLAICH, J., SCHOBER, H., Bahnbrücken am Lehrter Bahnhof in Berlin die Humboldthafenbrücke, Stahlbau, pp. 448-456, Ernst \& Sohn, 1999.

SCHLAICH, J., PÖTZEL, M., BEICHE, H., EHRKE, E., DECKER, U., Die Brücke über das Nesenbachtal im Zuge der Ostumfahrung StuttgartVaihingen, No. 11, pp. 678-687, Beton- und Stahlbetonbau 95, 2000.

SCHOBER, H., Rohrknoten aus Stahlguss, Der Prüfingenieur, pp. 16-36, October 2000 .

SCHUMACHER, A., Fatigue behaviour of welded circular hollow section joints in bridges, PhD thesis EPFL n ${ }^{\circ} 2727$, Swiss Federal Institute of Technology (EPFL), Lausanne, 2003.

SCHUMACHER, A., STURM, S., WALBRIDGE, S., NUSSBAUMER, A., HIRT, M.A., Fatigue design of bridges with welded circular hollow sections, Report ICOM 489E, Swiss Federal Institute of Technology (EPFL), Lausanne, 2003.

SDRC, I-DEAS (R), Structural dynamics research corporation (SDRC), 2000 Eastman drive, Milford, OH, U:S.A., 1999.

SEDLACEK, G., EISEL, H., HENSEN, W., KÜHN, B., PASCHEN, M., Leitfaden zum DIN Fachbericht 103 - Stahlbrücken, Ernst \& Söhne, March 2003.

SEEGER, T., Fatigue behaviour of HOESCH Gs Ark 10, Part 2: Fatigue design concept, Fachgebiet Werkstoffmechanik, Technische Hochschule Darmstadt, Report CSN 6, October 1981.

SEIFRIED, G., ANGELMAIER, V., WILHELM, G:, BESCHORNER, K., Eisenbahnbrücke über den Humboldthafen in Berlin, Stahlbau, Vol. 68, No. 7, pp. 511-519, 1999.

SIA 161/1: Stahlbauten, Schweizerischer Ingenieur- und Architektenverein, Zürich, 1990. 
[SIA 260]

[SIA 261]

[SIA 263]

[Sih et al. 1974]

[SN EN 12680-1:2003]

[SN EN 12680-2:2003]

[SN EN 12681:2003]

[SN EN 1369:1997]

[Sonsino et al. 1988]

[Stahl-Eisen-Prüfblatt 1922:1985] Ultraschallprüfung von Gusstücken aus ferritischem Stahl, StahlEisen-Prüfblatt 1922, 3. Ausgabe, Verein Deutscher Eisenhüttenleute, June 1985.

[Stahl-Eisen-Prüfblatt 1935:1982] Magnetpulverprüfung. Oberflächenprüfung von Gussstücken aus Stahl, Stahl-Eisen-Prüfblatt 1935, 1. Ausgabe, Verein Deutscher Eisenhüttenleute, June 1982.

[Stahl-Eisen-Prüfblatt 1936:1982] Eindringprüfung. Oberflächenrissprüfung von Gussstücken aus Stahl, Stahl-Eisen-Prüfblatt 1936, 1. Ausgabe, Verein Deutscher Eisenhüttenleute, June 1982.

[Stucki et al. 2000]

[SZS 2005]

[Trumpold et al. 1996]

[UEG 1985]

SIA 260: Grundlagen der Projektierung von Tragwerken, Schweizerischer Ingenieur- und Architektenverein, Zürich, 2003.

SIA 261: Einwirkungen auf Tragwerke, Schweizerischer Ingenieur- und Architektenverein, 2003.

SIA 263: Stahlbau, Schweizerischer Ingenieur- und Architektenverein, Zürich, 2003.

SIH, G. C., CHA, B. C. K., A fracture criterion for three-dimensional crack problems, Engineering fracture mechanics, Vol. 6, pp. 699-723, Elsevier, 1974.

Giessereiwesen - Ultraschallprüfung - Teil 1 : Stahlgussstücke für allgemeine Verwendung, SN EN 12680-1, SNV, January 2003.

Giessereiwesen - Ultraschallprüfung - Teil 2 : Stahlgussstücke für hoch beanspruchte Bauteile, SN EN 12680-2, SNV, March 2003.

Giessereiwesen - Durchstrahlungsprüfung, SN EN 12681, SNV, March 2003.

Giessereiwesen - Magnetpulverprüfung, SN EN 1369:1997, SNV, July 1997.

SONSINO, C. M., UMBACH, R., Gegenüberstellung verschiedener Fertigungs- und Konstruktionsmöglichkeiten von schwingbeanspruchten Rohrknoten bei Offshore-Bauwerken, Wirtschaftliche Bauteilgestaltung: Giessen, Schmieden, Sintern, Schweissen: Die Bedeutung der Prüftechnik bei der Bauteiloptimierung. Vorträge: DVM-Tage 1988: Bauteile '88, Deutscher Verband für Materialprüfung e. V., 1988.

STUCKI, D., DAUNER, H.-G., The Baregg bridge near Baden, 16. IVBHKongress Luzern, September 2000.

SZS, Espace de transit, steeldoc, No. 01/05, Stahlbau Zentrum Schweiz, 2005.

TRUMPOLD, H., PAPENFUSS, K., Empfehlung für die Einführung der Profiltiefe Pt zur Beschreibung der Oberflächenrauheit von Gusserzeugnissen, konstruieren + giessen, Vol. 21, No. 3, pp. 17-21, 1996.

UEG, Design of tubular joints for offshore structures, Part F.6: Cast joints, Underwater engineering group, Publication UR33, Vol. 3, 1985. 
[Walbridge 2005]

[Webster et al. 1980]

[Wood 1993]

[Wood et al. 1982]

[Zahoor 1985]
WALBRIDGE, S., A Probabilistic Fatigue Analysis of Post-Weld Treated Tubular Bridge Structures, PhD thesis EPFL n³330, Ecole Polytechnique Fédérale de Lausanne (EPFL), Lausanne, 2005.

WEBSTER, S. E., WALKER, F., WOOD, A. M., Cast steel nodes - their manufacture and advantages to offshore structures, European offshore petroleum conference \& exhibition, No. EUR 238, pp. 255-260, 1980.

WOOD, A. M., The efficient use of structural castings for lifted jackets, North sea innovations and economics, Proceedings of the institution of civil engineers, London, January 1993.

WOOD, A. M., GANTKE, F., WEBSTER, S., HANEKE, M., Recent advances in the technology of cast steel nodes for use in offshore structures, Annual offshore technology conference, No. 4, pp. 359-364, OTC 4198, 1982.

ZAHOOR, A., Closed form expressions for fracture mechanics analysis of cracked pipes, Journal of pressure vessel technology, Vol. 107, pp. 203-205, 1985. 



\section{SACHVERZEICHNIS}

Algorithmus, 114

Anfangsfehlergrösse

Ergebnisse mit Programm BEASY, 101

zulässige, 75, 94, 112, 114

Anlassen, 34

Ausnutzung unter Ermüdung, 113, 115

Ausnutzung unter Verkehr, 112, 115

Bauteiltemperatur, 83

BEASY

Programm, 67, 85

Belastungszustand

Einfluss des, 115

Bestimmtheitsmass, 107

Bezugstemperatur, 32

Bruchzähigkeit

ebener Dehnungszustand, 93

ebener Spannungszustand, 93

Einfluss der, 116

quasi-statische, 91

crack opening displacement, 67

Determinationskoeffizient, 107

Durchriss, 94

Durchstrahlungsprüfung, 14, 17

Einflusslinie

infolge Ermüdungslast, 84

Einschluss

nichtmetallischer, 12

Elektrode mit basischer Umhüllung, 35

Erschmelzungsprozess, 34

Fachwerkbrücke mit Stahlgussknoten, 10

Fachwerkbrücken mit Stahlgussknoten, 76 mit Stahlgussknoten, 2

Fachwerkträger, 29

Failure Assessment Diagramm, 93, 114

Farbeindringprüfung, 14, 17

Fehlergrösse massgebende, 75, 92, 94

Gasblasen, 11

Gussfehler, 11

Gussfehleratlas, 11

Gütestufe, 16, 35

Härten, 34

Kaltriss, 13

Kohlenstoffäquivalent, 33

Korrekturfaktor

Innenriss, 108

konstanter, 104

Oberflächenriss, 108

Kurzrissstadium, 91

Langriss-Bruchmechanik, 91

Lebensdauer

charakteristische, 91

Lichtbogenhandschweissverfahren, 36

Liefernorm, 16
Lunker, 13, 79

Magnetpulverprüfung, 14, 17

Masterkurve für GS-20 Mn $5 \mathrm{~V}, 32$

Metall-Schutzgasschweissverfahren, 36

minimum strain energy density criterion, 67

Modellfachwerkbrücke, 77, 78, 79

Ermüdungsbemessung, 80

Gebrauchstauglichkeit, 80

Tragsicherheit, 80

Nennspannung, 23

Nutzungsdauer, 91

Oberflächenriss

halbkreisförmig, 96

Parameterstudie, 112

Ergebnisse, 115

Paris-Erdogan Gleichung, 94, 114

Probekörper, 29

Prüfverfahren, 14

Randelementmethode, 66 duale, 67

Regressionsgerade, 107

Riss, 13

Rissschliesseffekt, 95

Rissspitze plastische Zone, 95

Risswachstumssimulation, 86

Rückrechnung auf zulässige Anfangsgrösse der Gussfehler Prinzip der, 92

Satz von Betti, 66

Schweissbadsicherung, 36

Schweisseignung, 33

Schweissnahtreparatur, 41

Schweissnahtvorbereitung, 36

schwitzwassertrocken, 35

Sekundärbiegemoment, 38, 42

Spannungsintensitätsfaktor, 96

Ergebnisse mit Programm BEASY, 97, 99

Näherungsformel, 104

Speiser, 13, 31

Sprödbruchnachweis, 83, 94, 99, 113

Stahlguss GS-20 Mn 5 V, 31

Stahlgussknoten

Modellierung, 79

Steifigkeit relative, 45

Ultraschallprüfung, 14, 17

VARP ${ }^{\oplus}$-Verfahren, 34

Vergüten, 34

Versuchsaufbau, 39

Versuchsprogramm, 38

Visual Basic for Applications, 114

Vorgehen zur Ermüdungsbemessung von Stahlgussknoten, 103,124

Vorwärmetemperatur, 33

Vorwärmung, 35

Wärmebehandlung, 34

Warmriss, 13 



\section{A. FABRIKATIONSPLÄNE SERIE B}
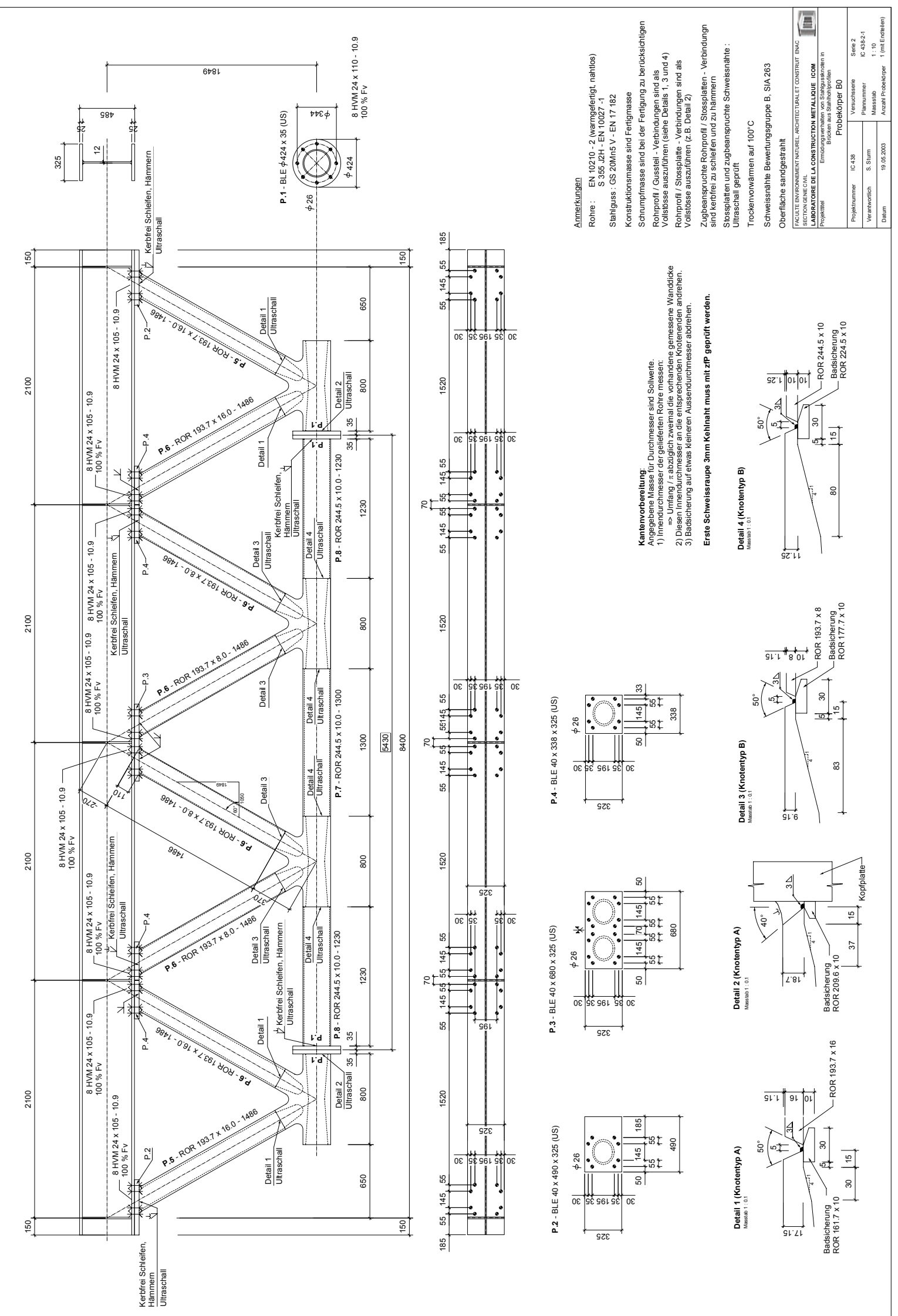

$\because \quad 988$ 

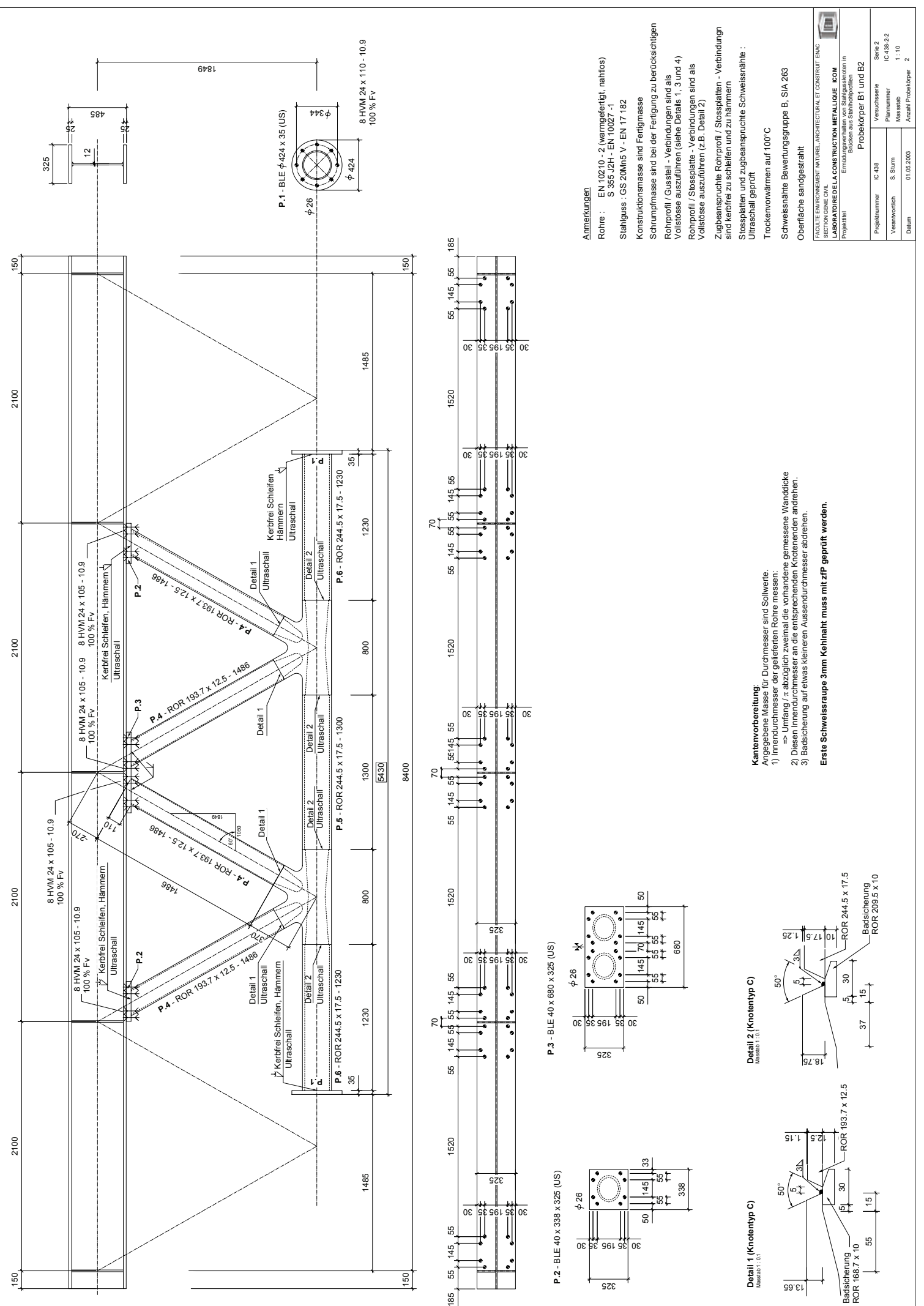
B. TABELLARISCHE AUFLISTUNG DER VERSUCHSDATEN

\begin{tabular}{lccccc}
\hline Probekörper & Schweissdetail & SCF [-] & $\Delta \sigma[\mathrm{MPa}]$ & $\Delta \sigma_{\text {SCF }}[\mathrm{MPa}]$ & $N[-]$ \\
\hline Voruntersuchung & Diagonale $^{1}$ & 1.02 & 77 & 78.5 & 145000 \\
& Untergurt & 1.17 & 147 & 172 & 164100 \\
\hline Probekörper A1 & Diagonale $^{1}$ & 1.02 & 67 & 68.3 & 398500 \\
& Untergurt (Fig. 3.4) & 1.17 & 154 & 180 & 200300 \\
& Untergurt (Fig. 3.4) & 1.17 & 123 & 143 & 200300 \\
\hline Probekörper A2 & Untergurt (Fig. 3.4) & 1.17 & 100 & 117 & 398500 \\
\hline Probekörper B0 & Untergurt & 1.17 & 137 & 160 & 167900 \\
& Untergurt (Fig. 3.6) & 1.16 & 137 & 160 & 223900 \\
\hline Probekörper B1 & Untergurt (Fig. 3.6) & 1.16 & 133 & 154 & 673200 \\
\hline Probekörper B2 & Untergurt & 1.098 & 86 & 95 & 1220600 \\
\hline
\end{tabular}

${ }^{1}$ Diagonale im Versuchsrahmen

${ }^{2}$ Abbruch des Versuchs ohne Versagen 


\section{ALGORITHMUS ZUR BERECHNUNG DER MAXIMAL ZULÄSSIGEN ANFANGSFEHLERGRÖSSE}

Option Explicit

Function MassgRissgroesse (f_y As Double, f_u As Double, ausn_verkehr As Double, Y_c As Double, R_e As Doublē, $t$ As Double, ${ }_{-}$mat As Double) As Variant

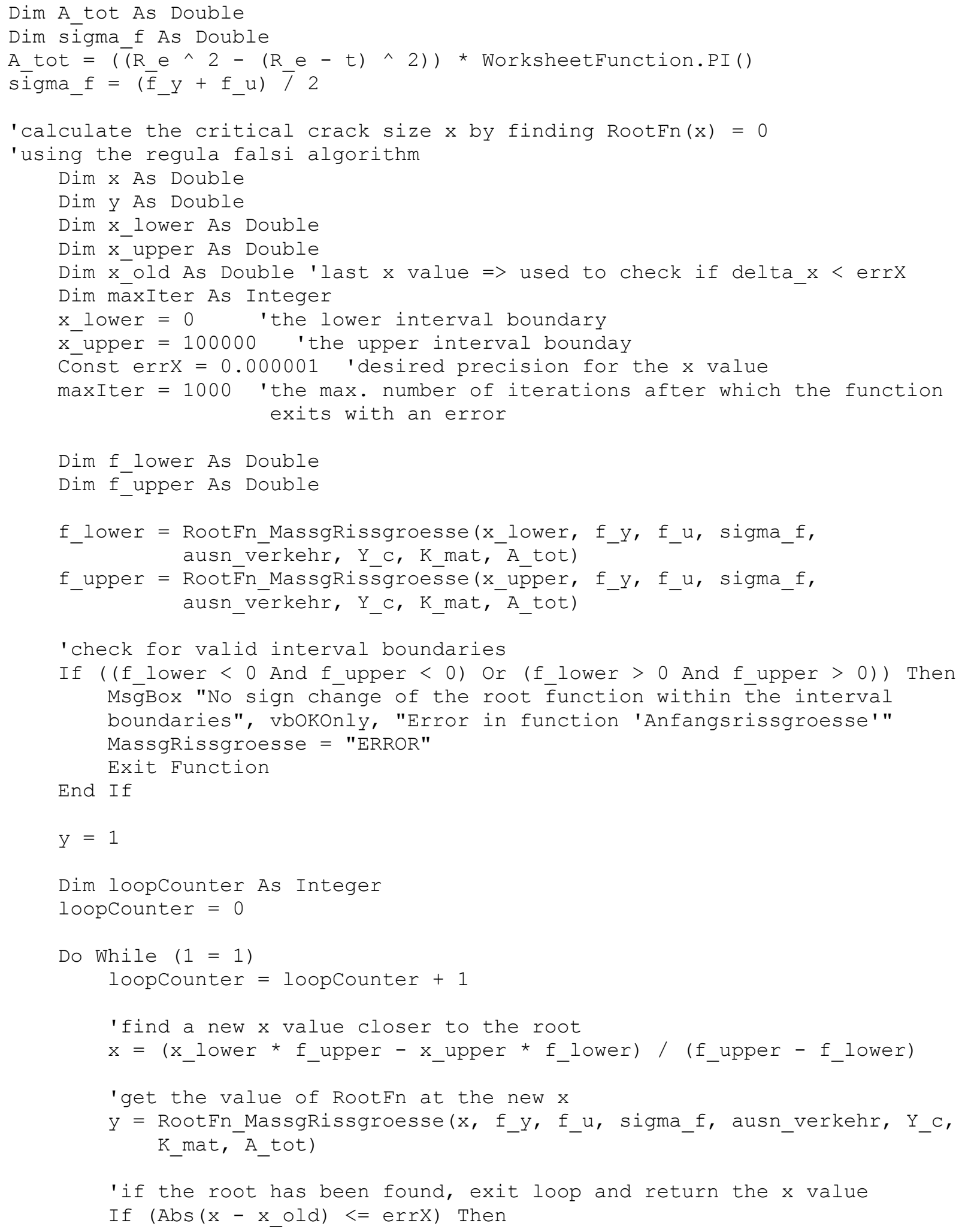




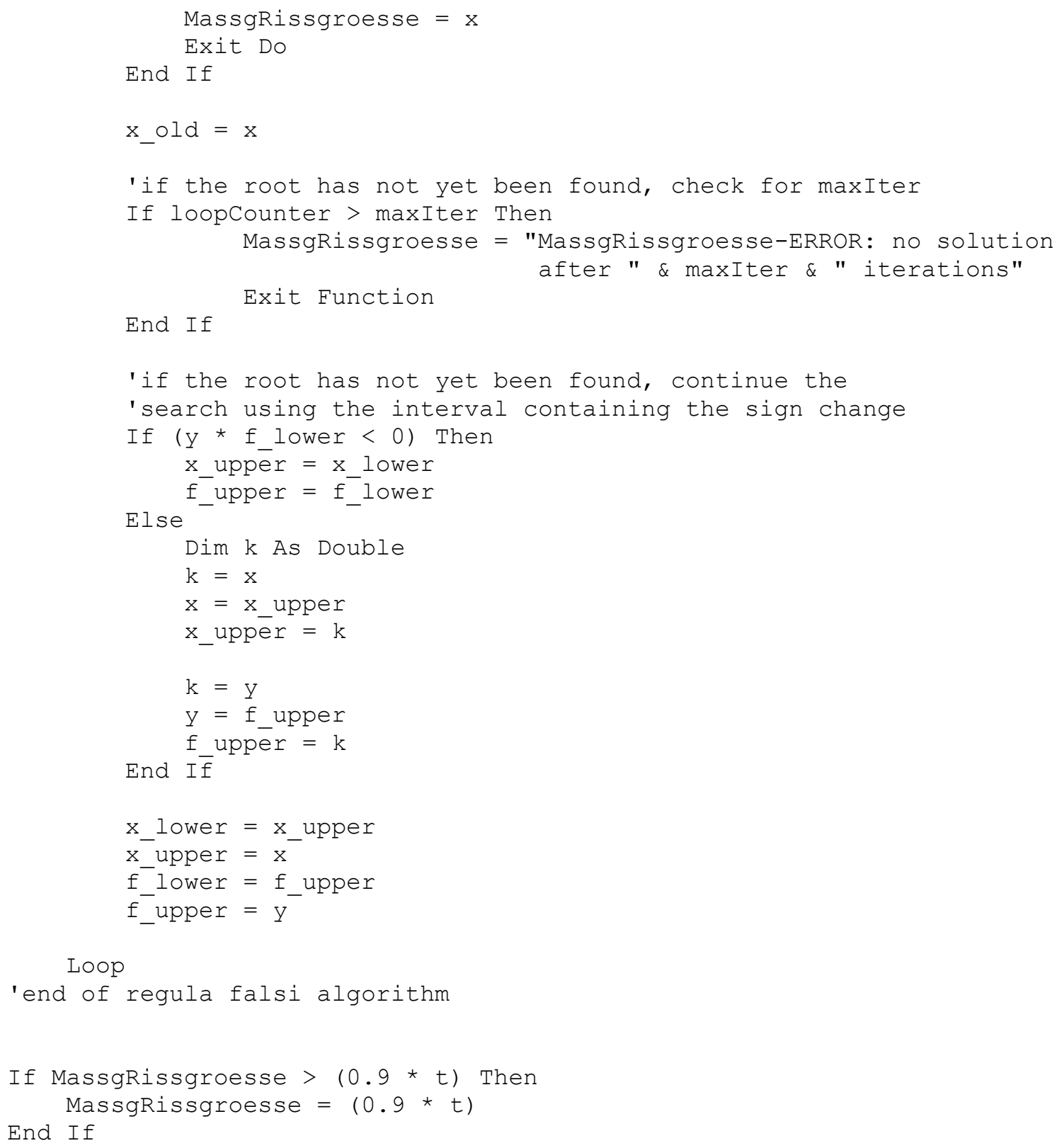


Function Anfangsrissgroesse (f_y As Double, ausn_ermüdung As Double, Y_a As Double, C As Double, m As Doub̄le, a_massg As Doüble) As Variant

'calculate the initial crack size $x$ by finding RootFn(x) = 0

'using the regula falsi algorithm

Dim x As Double

Dim y As Double

Dim x_lower As Double

Dim x_upper As Double

Dim x_old As Double 'last $x$ value $=>$ used to check if delta_x $<$ errx

Dim maxiter As Integer

$\mathrm{x}$ lower $=1 \quad$ 'the lower interval boundary

ReTryNewXL̄ower:

$\mathrm{x}$ _upper $=1000$ 'the upper interval bounday

Const errx $=0.000001$ 'desired precision for the $\mathrm{x}$ value

maxiter $=10000$ 'the max. number of iterations after which the function exits with an error

Dim f lower As Double

Dim f_upper As Double

f_lower = RootFn_Anfangsrissgroesse(x_lower, f_y, ausn_ermüdung, Y_a, C, $m$, a_massg)

f_upper $=$ Root $\bar{F} n \_$Anfangsrissgroesse (x_upper, f_y, ausn_ermüdung, y_a, C, m, a_mässg)

'check for valid interval boundaries

If ( $(f$ lower $<0$ And f_upper < 0 ) Or (f_lower $>0$ And f_upper $>0)$ ) Then If $^{-}(\mathrm{x}$ lower $=1)$ Then

$x$ lower $=0.0001$

GoTo ReTryNewXLower

Else

'MsgBox "No sign change of the root function within the interval boundaries", vboKOnly, "Error in function 'Anfangsrissgroesse'" Anfangsrissgroesse = "Anfangsrissgrösse-ERROR: No sign change of the root function within the interval boundaries" End If

End If

$y=1$

Dim loopcounter As Integer

loopCounter $=0$

Do While $(1=1)$

loopCounter $=$ loopCounter +1

'find a new $x$ value closer to the root

$\mathrm{x}=\left(\mathrm{x} \_\right.$lower * $\mathrm{f}$ _upper - $\mathrm{x}$ _upper * $\mathrm{f}$ _lower) / (f_upper - f_lower)

'get the value of RootFn at the new $x$

$y=$ RootFn_Anfangsrissgroesse (x, f_y, ausn_ermüdung, Y_a, C, m, a massg)

'if the root has been found, exit loop and return the $x$ value

If (Abs $(x-x+o l d)<=$ errX) Then

Anfangsrissgroesse $=x$

Exit Do 


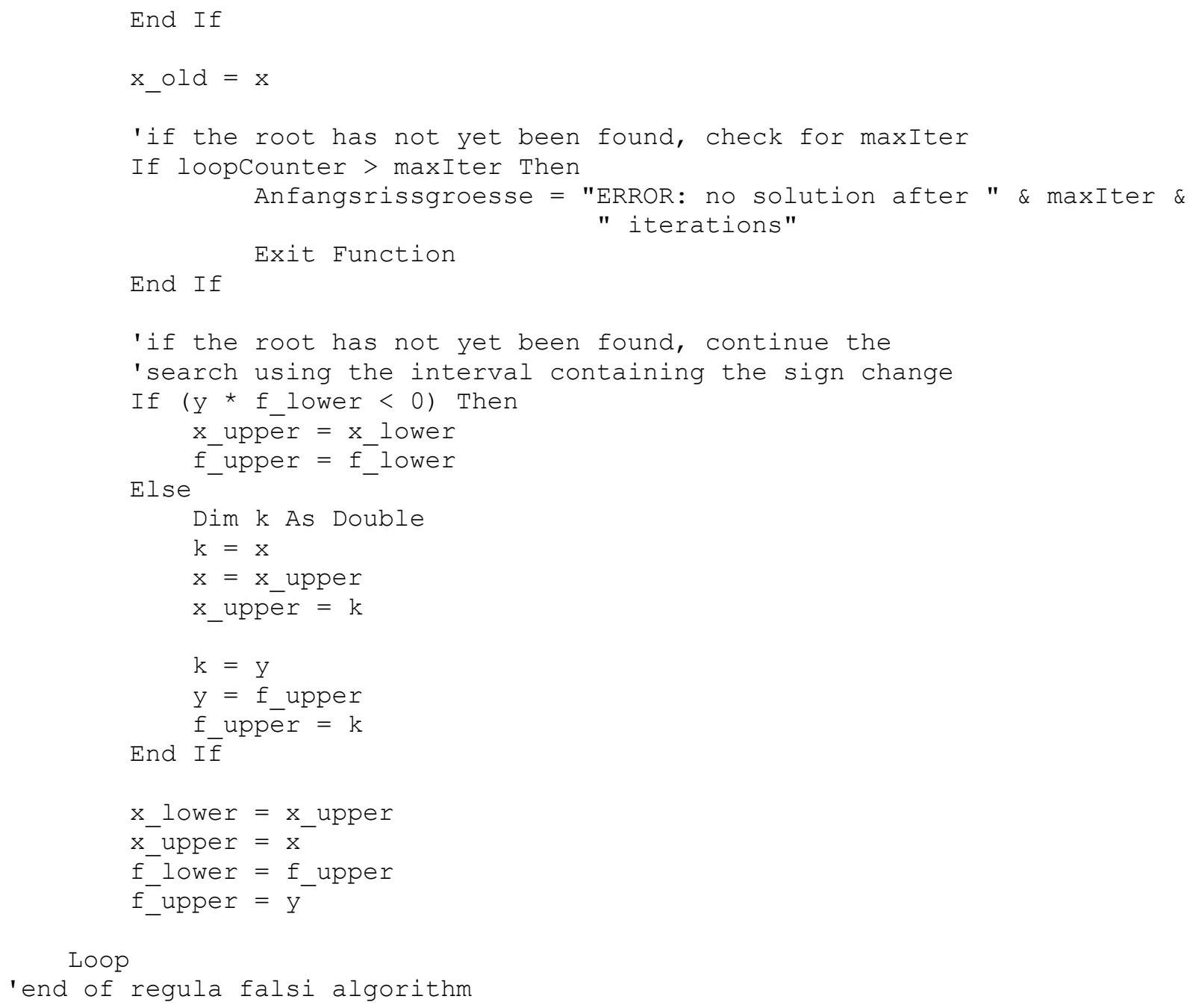

End Function

'The root of this function is at a = initial crack size

Function RootFn_Anfangsrissgroesse(a_0 As Double, f_y As Double, ausn_ermüdung As Double,

Y_a As Doub $\bar{l} e, c$ As Double, m As Double, a_massg As Double) As Double

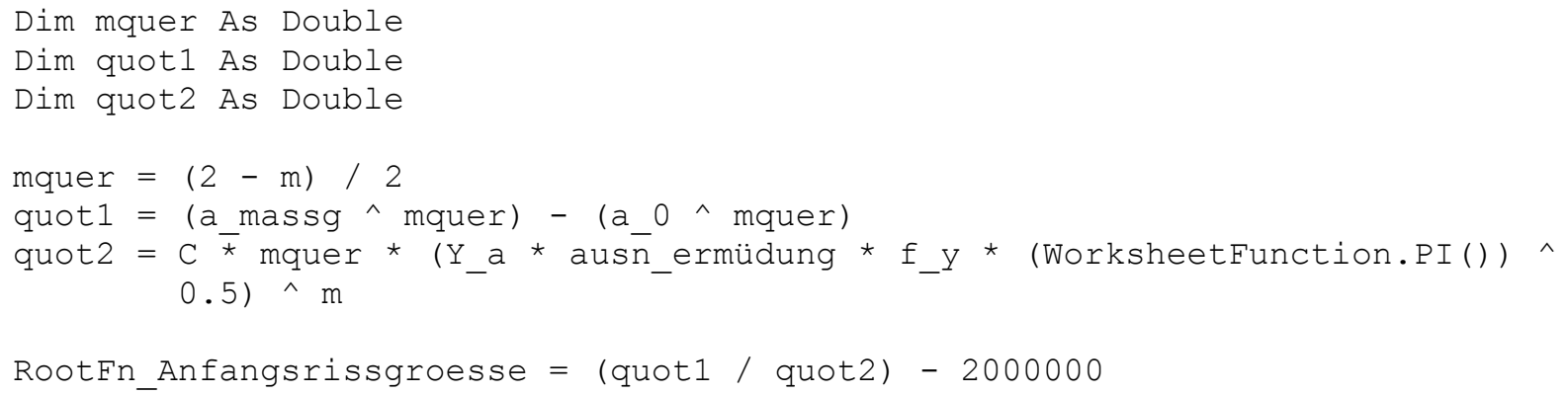

End Function 



\section{CURRICULUM VITAE}

Name

Geburtsdatum

Nationalität

Ausbildung

Berufserfahrung

Mitgliedschaft
Senta Christine Haldimann-Sturm

27. Februar 1975

Deutsch

2002 - Gegenwart

Doktorandin am Lehrstuhl für Stahlbau (ICOM) der Ecole Polytechnique Fédérale de Lausanne (EPFL), Lausanne, Schweiz

2003

Diplom als Europäischer Schweissfachingenieur (EWE, IIW), SLV Duisburg, Deutschland

2000

Diplom als Bauingenieur (Dipl. Bau-Ing. ETH), Ecole

Polytechnique Fédérale de Lausanne (EPFL), Lausanne, Schweiz

1994 Matura, Deutsches Gymnasium Biel-Bienne (Typus C, Mathematik/Naturwissenschaften), Biel, Schweiz

2000 - Gegenwart

Forschungsingenieurin am Lehrstuhl für Stahlbau (ICOM), Ecole Polytechnique Fédérale de Lausanne (EPFL), Lausanne, Schweiz

1998 - 1999 Hilfsassistentin am Lehrstuhl für Stahlbau (ICOM), Ecole Polytechnique Fédérale de Lausanne (EPFL), Lausanne, Schweiz

$1998-1999$ Hilfsassistentin am Lehrstuhl für Stahlbetonbau (IS-BETON), Ecole Polytechnique Fédérale de Lausanne (EPFL), Lausanne, Schweiz

Internationale Vereinigung für Brückenbau und Hochbau (IABSE) 